


\section{6}

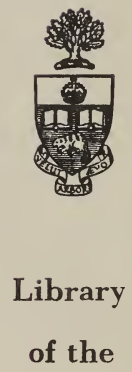

University of Toronto 

T H E

ELEMENTS OF CHEMISTRY. 



\title{
ELEMENTS OF CHEMISTRY,
}

\author{
FAMILIARLY EXPLAINED
}

AND PRACTICALLY ILLUSTRATED.

\section{ATTRACTION-HEAT-LIGHT-ELECTRICITY.}

"A knowledge of the principles of science makes men more skilful, expert, and useful in the particular kinds of work by which they are to earn their brearl, and by which they are to make it go far and taste well, when earned: it also gives every man a chance, according to his natural talents, of becoming an improver of the art he works at, and even a discoverer in the sciences connecter with it."

\section{O N D O N :}

JOHN MURRAY, ALBEMARLE STREET. 


\section{LONDON:}

PRINTED BY WILLIAM CLOWES, Stamford Street. 


\section{CONTENTS.}

INTRODUCTION

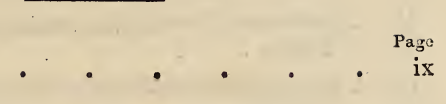

CHAPTER I.

Of the Objects of Chemistry, and of the Generai. Results of Attraction upon the various Forms of MatTer.

1. Of the general objects of Chemical Science .

2. Influence of Attraction upon the mechanical forms of Substances . . . .

3. Influence of Attraction upon the chemical composition of Substances . • • .

\section{CHAPTER II.}

Heat-its Effects and Sources.

$\S$ 1. Of Expansion in general . . . . . 34

2. Of the Expansion of Solids . . . 36

3. Experiments shewing the expansion of Liquids -Anomaly in regard to Water-Currents and their effects. . . .

4. Expansion of aëriform Bodies-Currents in the Air-Theory and practice of Ventilation .

5 Of the construction and uses of Thermometers and Pyrometers, and of the Scales applied to them 
$\S 6$. Of the relation of the Temperature of Bodies to the quantity of Heat contained in them

7. Solids, Liquids, and Gases, considered as conductors of Heat

8. Of the conducting Powers of Liquids .

9. Of the conducting Powers of Air in regard to Heat .

10. Remarks on the relative conducting Powers of Bodies .

11. Of the Effects of Heat in changing the forms of Bodies

12. Of the Steam Engine

13. Of radiant Matter . . . . . 112

14. Of the chemical Effects of radiant Matter . 114

15. Of the heating Effects of radiant Matter . 117

16. Of Terrestrial Radiation . . . . 118

17. Of the Effects of Radiation in Nature . . 134

18. Of Phosphorescence . • • . . 138

19. Of Ignition and Combustion . . . 145

\section{CHAPTER III.}

Electricity-its Sources and Effects.

§. Of Electrical Excitation-Attraction and repulsion

2. Of Electrical Conductors and Nonconductors . 167

3. Of Induced Electricity _ • • • 169

4. Of Electroscopes and Electrometers - . 173

5. Of Electrical Machines . • • . 179

6. Further Illustrations of Electrical Attraction, Repulsion, and Induction 
$\S 7$. Of the Dissipation and Reception of Electricity

Page by Points

8. Of Induction through Glass-Construction of the Leyden Jar

9. Of the Electrophorus • . . . 203

10. Of Galvanism or Voltaic Electricity - . 207

11. Of the Chemical Effects of Electricity - . 235

12. Of the Identity of the different forms of Electricity - . . . . . 258

13. Of the Source of the Electricity of the Voltaic Apparatus . . . . . 263

14. The Theory of Natural Phenomena as connected with Electricity . . . 268

15. Of Animal Electricity • • • • . 293

16. Of the Connexion of Electricity with Magnetism 298

INDEX 



\section{INTRODUCTION.}

THE Author has often been requested to furnish the young Chemist and general reader with some assistance to his studies, less learned and elaborate than the usual systematic works, and at the same time more detailed, connected, and explicit than the 'Conversations' and 'Catechisms.'

The present volume is a sample of such an attempt, and will be followed by others, containing the more practical and ordinary details of the chemical laboratory, and concluding with a general history of the rise and progress of the science. It includes an outline of the subjects of Atrraction, Heat, Light, and Electricity, in which he has endeavoured to avoid all prolixity of language and the use of the less intelligible technical terms.

It unfortunately happens that the matters treated of in this preliminary volume, are those of which it is most difficult to give a popular and condensed abstract, and they necessarily include references to other branches of chemistry with which it is the Author's business to consider the reader as unacquainted; but it will be requisite, on future occa- 
sions, so frequently to refer back to the principles here expounded, that he has rather hazarded the character of perspicuity at the outset, than commence with those departments of the science, which, although more popular and intelligible than the present, cannot possibly be either clearly explained or distinctly understood without constant reference to the preliminary information and general doctrines which are treated of in this volume.

It is obvious that the chemist can make very little progress in any of his inquiries, without constant reference to the general doctrines and individual laws of Atrraction, and consequently an outline of that subject forms the first chapter of the present volume. Its influence upon the forms and composition of bodies is generally explained, and we have endeavoured to show the manner in which mechanical and chemical attraction affect each other, and the links by which they are connected. To the superficial observer it may appear little else than matter of idle curiosity, to examine into the arrangements of the particles of crystalline bodies; to enumerate the different figures which different substances assume; to point out the varieties to which they are subject; and the cause of their modifications; but the fact is, that these apparently abstract inquiries abound in useful practical applications. The mineralogist who has studied crystallography, ascertains the names and 
nature of mineral products by a careful determination of their mechanical forms; the chemist, from the same source, is enabled in many instances to pronounce accurately upon the composition of bodies, without the time and labour requisite for analysis ; he whose business it is to cut and polish gems, is taught, in the same school, the directions in which those hard and refractory bodies split and polish, and the most economical methods of cutting and trimming them for ornamental purposes; and, transferring similar knowledge to the texture of rocks and mountains, the varieties of limestone are cleft asunder, and even the most indurated varieties of granite split into regular masses of portable and useful dimensions; from it the architect is taught the most durable arrangement of his materials; and the sculptor, knowing the texture of his marble, avails himself of such information in guiding his chisel and perfecting his work.

We next proceed to consider Attraction as influencing, not the mere form and texture, but the composition of bodies; its results in such cases are enumerated under the general term CHemical Attraction or Affinity, and here it is the fertile source of all those different qualities of bodies which depend upon the varied arrangements of their component parts.

Among the most remarkable consequences of 
chemical attraction, we may advert first to the striking dissimilarity which generally subsists between the properties of a compound and its component parts; and hence one of the greatest sources of interest belonging to chemical science, and a powerful incentive to the prosecution of experimental research. Substances of no value in themselves, combine to form compounds essential to the useful or ornamental arts; and from compounds that are to all appearance useless and unproductive, the chemist extracts valuable elements ; and all this, by his knowledge and management of the reciprocal attractions of bodies for each other. The ACIDS are well known as a set of most powerful and corrosive agents; but if we dissect them into their elements, we find these, with few exceptions, comparatively inert, inactive bodies. This is remarkably the case with two of the most useful and powerful of the class; namely, sulphuric acid, or oil of vitriol, and nitric acid, or aquafortis. The ALKalies are much in the same predicament; potash, soda, and ammonia, are noted for their activity and acrimony ; yet, when these enter into chemical combination with the acids, they constitute neutral salts, a class of bodies which, compared with the activity of their elements, are mild and harmless.

But the most remarkable instances of change in the qualities of compounds, and the most unequi- 
vocal proofs of our utter inability to discover them by any other than purely experimental methods, are derived from the chemistry of the organic creation: vegetable and animal products are constituted, as far at least as we know, almost exclusively of four elementary bodies, in themselves inert, insipid, inodorous, and insoluble; these are carbon or charcoal, and the three simple gaseous bodies, called hydrogen, oxygen, and nitrogen. These, in binary, ternary, or quaternary combinations, give rise to almost innumerable compounds, gifted with the most opposite properties, and apparently deriving these from the mere circumstance of slight differences in the relative proportions in which these four elements are united: thus, we recognise them in the fragrancy of the flower, and the fumes of putrefaction; in sugar, gall, and verjuice; in the nutritive seeds which form so important a part of our food; and in those poisonous products, of which a single grain produces death.

Inquiries connected with these subjects form some of the most useful and interesting objects of chemical research; they are obviously connected with those remarkable transmutations, or as it were, metamorphoses of matter, which are continually going on around us. The simple ingredients just adverted to, furnished by the air and soil, give rise to the infinite variety of vege- 
table products; and, in animal bodies, the various secretions, and the products of their growth, are derived from the same elements, combined and associated under the chemico-vital influence of assimilation and digestion.

In its applications to the useful arts of life, and to the investigation of inorganic compounds, chemistry furnishes no less interesting applications. The tanner converts perishable skin into permanent leather, the dyer confers durability upon the most transient colours, and the bleacher whitens his goods by processes purely chemical ; and upon these, and other equally important arts, chemistry has not only thrown the light of explanation, but also of improvement. It is a study equally essential in all that relates to metallurgy, and the numerous branches of human industry arising out of the infinite applications and uses of the metals and their compounds; and here, and in other cases, it furnishes the physician with the most powerful remedies for the relief of disease.

There are many who willingly admit the great advantages that chemistry has conferred upon the arts, but are inclined to dispute its merits as an abstract and insulated study ; and who adduce the many useless discoveries, as they call them, in sanction of their contempt. But the progress of modern chemistry has, or ought to have, 
silenced all such objections. Those refined and abstruse inquiries, for instance, which have led to the establishment of the theory of definite pro. portionals, or, as it is often called, the Aтomic Theory, and which are apparently only applicable to the most delicate researches of the analyst, or to the elucidation of a few points of theory, have in fact proved of the utmost practical importance; they have opened new fields of discovery; they have furnished the manufacturer with an unequivocal test of the perfection of his processes, teaching him to economize his products and multiply their applications, and enabling him surely and satisfactorily to transfer the results of experiments upon a few grains of materials, to the enormous scale of commercial consumption.

In chemistry, all experience teaches us that every new discovery is to be hailed as the forerunner of important consequences, however useless and insignificant in its apparent origin. The metal chrome, discovered by Vauquelin, is in itself quite unimportant, but in its combinations it furnishes the painter and the calico-printer with some of their most rich and durable colours. The substance called iodine, accidentally detected in the refuse of soap-lees, and at first apparently a mere chemical curiosity, has proved a valuable and powerful medicine in the relief of glandular complaints; and the same history at- 
taches more or less to nearly all the most powerful and useful agents of technical chemistry; but what has been said ought to be sufficient to answer the objections of those who cavil at the unproductiveness of science, and many such there are, whose rank in life and influence in society render their example peculiarly baneful. The importance of Sir H. Davy's grand discovery of the metals of the alkalies, has been questioned upon such principles; but to say nothing of the remarkable properties which those bodies exhibit, of their importance as instruments in the hands of the experimental chemist, of the new views which their discovery has suggested, and of the subversion of erroneous theories which it has sanctioned, it is impossible to say to what useful purposes (in the most vulgar acceptation of that term) they may not hereafter be applied. Exactly the same line of argument was applicable to Scheele's discovery of CHLORINE : it was a curious and powerful agent, but apparently useless ; yet, in process of time, it completely revolutionized the important art of bleaching; it has been a source of enormous profit to the manufacturing chemist; and has more lately proved a means of disarming infectious and contagious diseases of their most prominent horrors.

To those who still assail the votaries of science with the question 'cui bono,' we strenuously recom- 
mend the attentive perusal of Mr. Herschel's ' Preliminary Discourse on the Study of Natural Philosophy.' The transformations of chemistry, he says, by which we are enabled to convert the most apparently useless materials into important objects in the arts, are opening up to us every day sources of wealth and convenience, of which former ages had no idea, and which have been pure gifts of science to man. 'Every department of art has felt their influence, and new instances are continually starting forth, of the unlimited resources which this wonderful science develops in the most sterile parts of Nature. Not to mention the impulse which its progress has given to a host of other sciences, what strange and unexpected results has it not brought to light in its application to some of the most common objects! Who, for instance, would have conceived that linen rags were capable of producing more than their own weight of sugar, by the simple agency of one of the cheapest and most abundant acids? - that dry bones could be a magazine of nutriment, capable of preservation for years, and ready to yield up their sustenance in the form best adapted to the support of life, on the application of that powerful agent, steam, which enters so largely into all our processes, or of an acid at once cheap and durable?-that sawdust itself is susceptible of conversion into a substance having no remote analogy to bread; and 
though certainly less palatable than that of flour, yet no way disagreeable, and both wholesome and digestible, as well as highly nutritive?'

Such are a few of the general points suggested by the consideration of the results of chemical affinity; a subject which, in the present volume, can of course only be very superficially touched upon, since the details relating to it are necessarily those of chemistry at large.

The second chapter of this volume relates to Heat, a subject of infinite importance to the chemist, who, indeed, was formerly exclusively considered as the ' philosopher by fire.' The reader's attention is here only directed to its general effects upon the different forms of matter; it is considered as an antagonist power to attraction; as tending to separate the particles of bodies, and so changing their states; as exciting peculiar sensations referable to changes of temperature; and as pervading bodies by its power of conduction, and traversing space by radiation; as emanating from the sun, associated with light, and with other highly refrangible rays possessed of certain peculiar chemical powers. The details of the effects of heat upon individual substances, are afterwards to be more fully and particularly explained, but its general history includes many points of popular application and interest, as relating especially to the means of economizing. 
fuel, of warming our habitations, of ventilating public buildings, and so forth.

Electricity is another power of matter, which late discoveries have shown us to be intimately connected with chemical changes; and as it will not be necessary again often to recur to its general phenomena and agencies, they are given somewhat in detail in the third chapter. In regard to this subject, much difficulty is necessarily experienced by the mere chemist, in saying enough to render its applications to chemistry perspicuous and intelligible, without entering into a variety of general and theoretical details, which, however essential to the electrician, are not so to the chemist; we have therefore endeavoured to be as explicit as is requisite upon those points which connect electrical with chemical science, omitting such details as do not come within these limits. Upon this principle, little is said upon that very curious, interesting, and important branch of inquiry which connects electricity with magnetism, and of which only so much is here admitted as may render the chemical use of one or two electromagnetic instruments intelligible.

The author must now again remind his reader, that the present volume is merely intended as an introduction to those which are to follow; as containing certain general doctrines and statements, which are afterwards to be more explicitly 
verified and illustrated: it is hoped that it may serve as a test or touchstone, by which a taste for chemistry may be detected; and that its perusal may induce the student to seek for further information in those authors who are less superficial and elementary. 


\section{ELEMENTS \\ oF \\ C H E M I S T R Y.}

CHAPTER I.

OF THE OBJECTS OF CHEMISTRY, AND OF THE GENERAL RESULTS OF ATTRACTION UPON THE VARIOUS FORMS OF MATTE?.

$\$ 1 .-O f$ the General Objects of Chemical Science.

Chemistry is a branch of science which teaches the properties of the elementary bodies, and of their mutual combinations. It inquires into the powers which affect, and into the laws which preside over, their union; it examines the proportions in which they combine, and the modes of separating them when combined; and endeavours to apply such knowledge to the explanation of natural phenomena, and to useful purposes in the arts of life.

'The foundations of chemical knowledge are, observation, experiment, and analogy. By observation, facts are distinctly and minutely impressed on the mind: by analogy, similar facts are connected : by experiment, new facts are discovered: and in the progression of knowledge, observation, 
guided by analogy, leads to experiment ; and analogy, confirmed by experiment, becomes scientific truth. To give an instance,-Whoever will consider with attention the slender green vegetable fibres of confervee which, in the summer, exist in almost all streams, lakes, or pools, under the different circumstances of shade and sunshine, will discover globules of air upon the filaments exposed under water to the sun, but no air on those that are shaded. He will find that the effect is owing to the presence of light. This is an obsercation; but it gives no information respecting the nature of the air. Let a wine-glass filled with water be inverted over the conferva; the air will collect in the upper part of the glass, and when the glass is filled with air, it may be closed by the hand, placed in its usual position, and an inflamed taper introduced into it: the taper will burn with more brilliancy than in the atmosphere. This is an experiment. If the phenomena are reasoned upon, and the question put, whether all vegetables of this kind, in fresh or in salt water, do not produce such air under like circumstances? - the inquirer is guided by analogy; and when this is determined to be the case by new trials, a general scientific truth is established-namely, that all conferve in the sunshine produce a species of air that supports flame in a superior degree *.'

To this illustration it may be added, that the pure air thus generated by aquatic plants under the influence of light, is essential to the respiration and health of fish, and other inhabitants of the water; and thus those animals soon become

* Davy's Elements of Chemical Philosophy. 
unhealthy, and die, in water destitute of living vegetables: hence we perceive that it is not only fresh water which they require, but that certain chemical changes are requisite to the continuance of their healthy functions, which are effected by the vegetable tribes under the agency of light. If we breathe the same portion of air for a short time only, it becomes unfit for the purpose, and a candle will not burn in it; it has become contaminated during respiration by a substance injurious to health: if this same air be exposed to the influence of growing plants, and of the sun's rays, it becomes gradually restored to its former state, and a candle will burn in it, and an animal may breathe it as before. These instances show that the functions of animal and vegetable life are dependent upon, and connected with, chemical changes. Other functions of animals, such as digestion, respiration, and secretion, are also distinct subjects of chemical inquiry.

But the chief business of the Chemist is with inanimate or inorganic matter, and its diversified arrangements in art and nature. He examines it, not, like the Natural Philosopher, with a view of determining its magnitude, its form, and other mechanical attributes, but he endeavours to ascertain its more intimate nature and properties: he soon finds that the different kinds or forms of matter, although apparently infinitely various, may be divided into two comprehensive classes: the one including simple or elementary bodies, such as the metals, \&c., and the other, compounds, such as air, water, \&c.

By the term simple substance or ELEMENT, mo- 
dern chemists do not imply, like those of old, certain imaginary principles, or supposed elements of all matter, such as fire, air, earth, and water; but they give that term generally to such substances as they cannot further decompose or resolve into simpler forms. None of the metals, for instance, have either been decomposed or composed; nor has sulphur, nor the diamond, nor many other bodies which shall afterwards be described: those, therefore, in the present state of our knowledge, are called elementary or simple substances; not that it is intended by those terms to imply that they may not be decomposed at some future period, but merely that we are yet ignorant of their more intimate nature or composition: for many substances once classed among the elements have since been decomposed: potash and soda, magnesia and lime, for instance, a few years ago were placed among the simple substances; now, we know that they are compounds, each consisting of two simple substances ; namely, of a metal and an aëriform body or gas * called oxygen.

In examining the elementary substances in detail, the chemist ascertains, not merely their abstract properties and uses, but he particularly examines the products which they form by combining with each other; he determines accurately the proportions in which they unite, and the properties and uses of the resulting compounds. With regard to compound bodies, these the chemist resolves into their elements : if products of nature, he ascertains how far they can be imitated by art, and how

* This term is generally applied in chemistry to all kinds of air differing from common air. 
far they are susceptible of useful applications to the purposes of the arts of life.

In his examination, for instance, of atmospheric air, the Chemist finds that it is a mixture of two kinds of air, or gases, eminently differing from each other: in the one, bodies burn with great splendour, and produce an immense quantity of heat and light ; in the other, on the contrary, all burning bodies are immediately and entirely extinguished; the one, therefore, supports and the other does not support combustion: in the former, animals live for a longer time than they do in an equal bulk of common air ; in the latter, they instantly die. Now it is to the due admixture of these two gases, in certain proportions, that the properties of the air we breathe are to be referred, the one modifying and influencing the effects of the other. The gas which supports combustion and life, and which, therefore, has been called empyreal or vital air, has also been shown to exist in many of the acids: hence, in modern chemical nomenclature, it is called oxygen gas, a term derived from the Greek, and signifying producer of acid; the other gas, which extinguishes flame, and which is fatal to animals, has been named azotic gas, or azote, a term also of Greek origin, and implying its destructive properties: it is also an ingredient in aqua fortis, or nitric acid, and has hence received the name of nitrogen. Atmospheric air consists of four-fifths of nitrogen and one-fifth of oxygen, these gases being in a state of mixture, and their relative proportions so admirably adjusted as to fit the atmosphere for the various important ends which it answers. If 
oxygen, for instance, were to predominate, our fuel of all kinds would burn with prodigious and alarming rapidity; even the iron of our grates would take fire; and all common combustibles would be consumed with intense heat and light: the vegetable world would also feel the change, and plants would become unhealthy or perish; and in the animal creation, a hurried circulation and febrile symptoms would precede dissolution. If the opposite element, or the nitrogen, were in excess, feeble respiration, or even suffocation, would ensue among animals; plants would languish; and all sources of artificial heat and light would be weakened or annihilated. We observe, therefore, with what infinite wisdom the mixture of these two elements of the air is so exactly adjusted and balanced, as precisely to answer its intended and ordained ends.

When nitrogen and oxygen, instead of being in a mere state of mixture, are chemically combined, they give rise to a variety of important compounds: among these, as already hinted, is aqua fortis, or nitric acid, the elements of which are nitrogen, oxygen, and water : into these principles it is resolvable by analysis ; and from these it may be compounded by the synthetical operations of nature and art.

In water the Chemist discovers two elementary bodies-oxygen is one; and the other has been called hydrogen, that is, producer of water: hydrogen is, moreover, an agent of great importance in nature and art, and is the lightest gaseous body known; hence it has been used for filling air balloons. The proportions in which these ele- 
ments are united in water are as eight to one, so that nine pounds of water contain eight pounds of oxygen and one of hydrogen.

In common salt we find a highly inflammable metal called sodium combined with chlorine, a body well known, in its gaseous state, as a powerful bleaching substance, and eminently destructive of infectious, contagious, and putrid matters. When these, the elements of salt, are united with those of water, the chlorine, combining with the hydrogen, forms that useful substance the $m u$ riatic acid, or spirit of salt; and the sodium, with the oxygen, gives rise to soda or the mineral alkali, an article essential in the manufacture of glass, soap, and other products of art.

These subjects are now merely touched upon in illustration of some of the terms and objects of chemistry; in another part of these essays they will be taken up in detail.

The leading chemical classification, then, of substances, is into simple and compound; but in pursuing his examination of these, the chemist finds it necessary also to make himself acquainted with the general history and effects of those powers of matter which preside over and modify chemical changes-such, for instance, as ATTRACTION, HEAT, and ELECTRICITY; and with these it is well to form a preliminary acquaintance, inasmuch as there is no operation of the laboratory in which they are not concerned. Our first object, therefore, will be to acquaint the reader with such facts respecting these attributes of matter as concern the chemist -premising that we know nothing whatever of their cause, and, consequently, our investigations are exclusively limited to an examination of their 
effects. If asked what attraction is, we say it is the effect of an unknown cause-and the same reply is applicable in the cases of heat and electricity : in regard to the latter, however, it is frequently the custom to talk of the matter of heat and of the electric fluid; but although it is often very convenient to assume, that heat and electricity depend upon the presence of some highly attenuated form of matter, it must always be remembered, that the existence of such matter has not been proved by experiment ; it is merely hypothetical ; and we are in reality as ignorant of the cause of the phenomena of heat and electricity, as of that of gravitation or attraction.

\$2.-Infuence of Attraction upon the Mechanical Forms : of Substances.

Atrraction is exerted at sensible and at insensible distances*, and among the similar and dissimilar particles of matter. A few words in further explanation of this subject will enable us to notice certain terms employed in chemistry, which may not, perhaps, be familiar to the reader, and to describe some of the commonest operations of the laboratory.

The phenomena of attraction acting at sensible distances, or of gravitation, we leave chiefly to the mechanical philosopher; but when the same power acts at insensible distance, it becomes an

* At sensible and often immense distance, as among the planetary bodies: at insensible distance, as where it retains the particles of a lump of chalk or marble adhering to each other, there being no visible space between them. 
immediate object of chemical inquiry. Acting upon the minute particles of matter, it influences their form and texture, and when exerted between dissimilar atoms it effects their union or combination, and is exclusively a subject of chemical research. The present section will contain some general details upon these subjects, which will afterwards be more fully explained and applied.

If we take a shapeless lump of alum and plunge it into a tumbler-full of cold water, we shall observe that in a few days the surface of that salt will be eaten and carved out into a variety of regular forms, somewhat as represented in the annexed cut; and

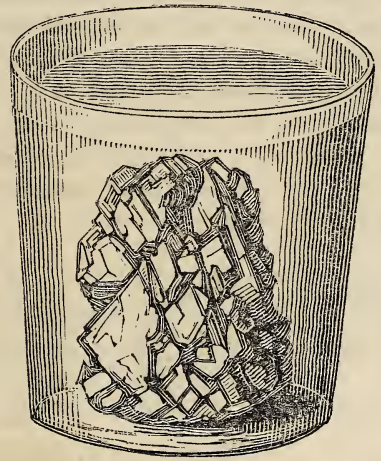

if we put a drop of the solution contained in the tumbler upon a plate of glass, we shall perceive, as the water slowly evaporates, that the particles of alum which it contained congregate into regular forms, which, when examined by a common pocket 
lens, appear as below, and are small and beautifully formed eight-sided figures, or octoedra. In this

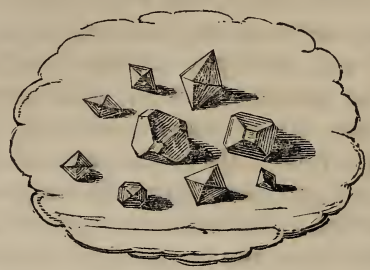

case the water is said to be the solvent of the alum, and the process by which it is obtained in regular forms or crystals, is called crystallization. In this and similar instances, the crystals are liable to certain modifications; thus among the little octoedrons, to which we have adverted, it is not uncommon to find some, the angles of which are cut off, or replaced by secondary surfaces; sometimes the edges too are similarly modified, and the crystals assume these figures.-

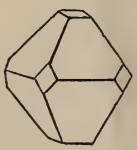

Truncated Angles.

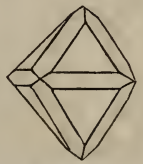

Truncated Edges.

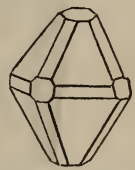

Truncated Edges and Angles.

Most of the saline substances soluble in water may be thus exhibited in a crystalline form; and as each substance usually assumes a distinct figure, or is characterized by some obvious, and, at the same time, peculiar properties, we are often 
enabled to pronounce upon the chemical nature or composition of a body, by a careful examination of its crystalline forms; and such experiments are particularly neat and satisfactory, in consequence of their facility, and the small scale upon which they may be performed: for the figures of the smallest crystals obtained from a drop of their solution, resemble the large ones formed in gallons of it; and although, in collecting specimens of crystallized salts, it is convenient to have them large and perfect, the smallest ones that may be viewed through a microscope furnish the chemist with equally satisfactory evidence of the nature of the dissolved salt:-for instance, put a tea-spoonful of common salt, nitre, Glauber's salt, and Epsom salt, into separate wine-glasses; fill them up with water, and occasionally stir their contents to help the solution: the next day take a drop of the clear liquor, with a glass rod, out of each of the wineglasses, and place them side by side upon a strip of clean plate glass, which may be placed upon a chimney-piece, or somewhere very gently warmed. As the water evaporates, the salts will crystallize, and we shall observe the following figures appropriated to each:- the common salt exhibits cubes - the Epsom salt, four-sided prisms - the nitre, sixsided prisms - the Glauber's salt, irregular six-sided prisms.

The Glauber's salt and the nitre might possibly at first be mistaken for each other, but, while the latter remains unaltered by exposure to air, the former soon loses its transparency, and crumbles down into white powder. Salts which behave thus are said to effloresce: other salts, instead of thus 


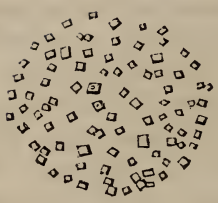

1. Common Salt.

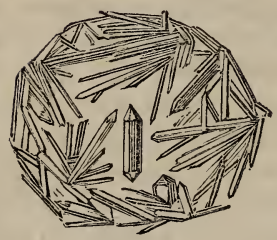

3. Nitre.

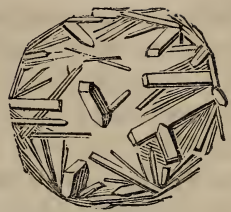

2. Epsom Salt.

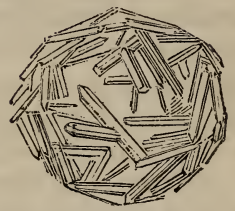

4. Glauber's Salt.

spontaneously parting with a portion of their water of crystallization, attract moisture from the air, or deliquesce, as is the case with carbonate of potassa, or, as it is commonly called, salt of tartar: alum and many other salts, although they contain water, do not thus part with it, and are permanent in the air. But water is not necessarily contained in all crystals : there is none, for instance, in nitre ; and such are called anhydrous salts*. Some salts require a high temperature, to deprive them of water. This is the case with gypsum or sulphate of lime, which is commonly fojund in large transparent crystals, permanent in the air; but at a red heat they lose one-fifth their weight of water, and crumble down into the white powder commonly

* Anhydrous is a term derived from the Greek, signifying without unter. 
called plaster of Paris, which is, therefore, an anhydrous sulphate of lime, and which, when mixed with a little water, again combines with it, and concretes in the act of combination-hence the use of this substance for forming casts or impressions, as seen in mouldings, busts, \&c.

It is a curious circumstance that some substances so powerfully attract water, as to retain it even at a red heat: hence some, who are fond of paradox, have maintained the existence of red-hot ice; for the water forms part of a solid, and solid water is ice. However, the fact is curious, as showing the power of chemical attraction in overcoming the strong propensity which water at high temperatures has to pass off in the state of steam.

The method of obtaining crystals by solution in water, and evaporation, is that commonly practised: when a lump of salt is thrown into the water, the attraction of aggregation existing among the saline particles is partly overcome by the attractive power of the water: in time, however, the water ceases to exert further action, and its attraction for the salt is counterbalanced by that of the particles of the salt among themselves: if we diminish this aggregation of the saline particles by the help of heat, more of the salt will for a time be taken up by the water, till the attractions again balance each other ; if we then drive off a portion of the water by heat, the particles of the salt will gradually attract each other, and solidify in the crystalline form. During this evaporation, it often happens that a film or pellicle of salt forms upon the surface of the hot liquor, indicating that the attraction of the particles of the salt for each other is overcoming their 
attraction for the water which previously held them dissolved : in this case, if the solution be set aside to cool, a large crop of crystals will ultimately be obtained, the size and perfection of which is generally dependent upon the care and slowness with which they have been suffered to form. Rapid evaporation, and agitation of the liquor, prevent the production of large and beautiful crystals; and

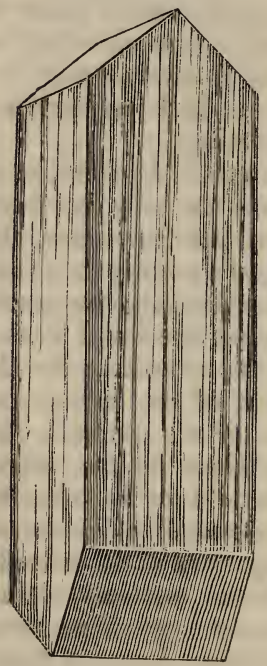

Epsom Salt, or Sulphate of Magnesia.

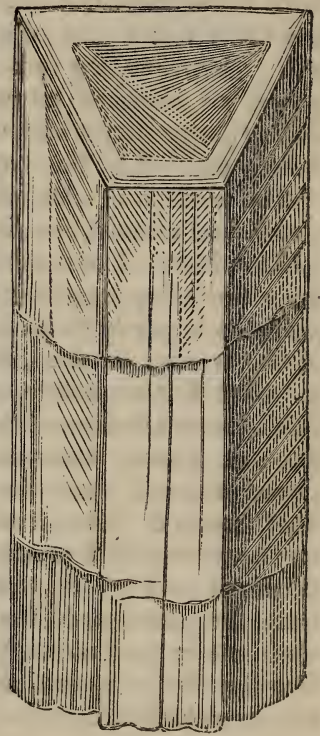

Saltpetre, or Nitrate of Potassa. 
though, upon the great scale in the arts, solutions of salts are often quickly boiled down to a concentrated state, and stirred to facilitate evaporation, by which the crystals are rendered small and irregular, yet, if we wish to procure them perfect and large, the evaporation should go on very quietly, and at low temperatures : Epsom salt, for instance, as commonly met with, is in very small broken and irregular crystals, owing to the haste with which the manufacturer prepares it; but if the solution be very slowly evaporated, or the water suffered to exhale spontaneously, crystals of singular regularity and beauty may be obtained.

The preceding wood-cuts represent crystals of Epsom salt and nitre, in my possession, of their natural size, and they might be procured even of yet more gigantic dimensions.

The above are a few of the general facts connected with the subject of crystallization as going on in aqueous solutions; but there are some remarkable circumstances affecting the process, and often interfering with the perfection of its results. One of these is the agency of light: put a solution of nitre, of proper crystallizing strength, (a pound of the salt dissolved in four pints of hot water,) into a glass basin, and so arrange it that the light may fall much more intensely upon one side of the basin than the other: it will then be found that the crystals form in much the greatest abundance upon the illuminated side, and that they are only sparingly deposited upon the dark side of the basin. If we examine the bottles containing camphor, in the windows of druggists' shops, we almost always remark a beautiful crystalline deposit of 
that substance upon the side which receives most light ; and in looking over the crystallizable preparations upon the shelves of a laboratory, many similar instances will be remarked. That light, therefore, favours and affects the tendency of bodies to assume crystalline arrangements, is beyond doubt; but what its specific action is, we know not.

There are other causes equally recondite, which affect crystallization. If a strong solution of sulphate of soda be left perfectly quiet, it may chance to remain liquid; but upon gently tapping the vessel which contains it, it shall suddenly shoot into a mass of crystals. Under the same circumstances, it will suddenly crystallize upon dropping into it a particle of solid matter, or a crystal of the salt. Indeed, solid bodies introduced into crystallizing solutions in general, aid the process ; they form nuclei, as it were, for the reception of the small crystals : thus we see sticks in the centre of crystallized verdigris; and strings drawn through the syrup intended to form sugar-candy, which is merely sugar in its most perfect crystalline form.

Another paradoxical experiment, in regard to crystallization, is the following :-Fill a small flask with a very strong and hot solution of Glauber's salt, and immediately cork it tight, or tie two or three folds of bladder over the mouth so as effectually to exclude the ingress of air: if in this state the flask be left perfectly quiet, it will remain for some days, "and its contents will show no tendency to crystallize; if violently shaken, crystals generally form, but it will sometimes even resist this operation: if, however, the cork be withdrawn, or the bladder punctured, so as suddenly to admit air, 
crystallization immediately commences upon the surface, and rapidly pervades the whole. If the air be very slowly admitted, the solution sometimes remains liquid for a considerable time, but instantly congeals upon shaking the flask, touching the liquid with a straw or wire, or throwing into it the minutest particle of any foreign matter.

The crystallization of substances not soluble in water may often be beautifully effected by the aid of heat, which gives freedom of motion to their particles in two ways : narnely, either by liquefying or melting them, or by converting them into vapour; and, as the liquid solidifies on cooling, or the vapour on condensation, crystals of more or less regularity are formed. To crystallize a metal in this way, melt it in a deep iron ladle or crucible, and when the surface cools, suddenly pierce it with an iron wire, and quickly pour out the liquid metal from the interior: when the whole has cooled, carefully

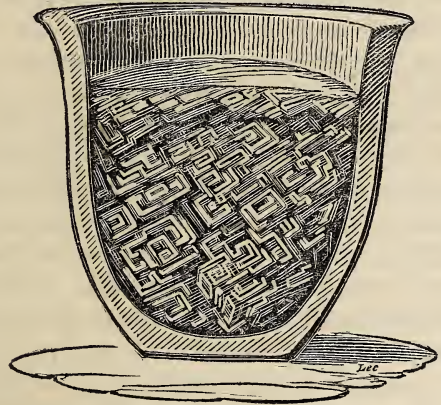

Crystallization of Bismuth. 
break the hollow ingot, and the cavity will be found lined with more or less perfect crystals. Bismuth is the metal which succeeds best for this experiment; but tin, lead, and antimony, also afford tolerably distinct crystals.

There is another way of showing the crystalline texture of metals, which, with some of them, answers very well. It consists in washing over a thin plate of the metal with some acid which acts slowly upon its surface. If tinfoil or tin plate be thus washed over with a mixture of one part of sulphuric, and one of muriatic acid, with twelve of water, the crystallization of the tin is often rendered beautifully manifest: this has been applied to ornamental purposes, under the name of moirée métallique, and different colours are easily given to the reflecting surface of the metal by the application of transparent varnishes.

When bodies are raised in vapour, and this is again condensed into the solid state, the process is generally called sublimation, and is not uncommonly resorted to to obtain such volatile matters in the form of crystals. The appearance. of camphor under such circumstances has already been noticed. Iodine is another body well calculated for the experiment: put a little of it into a clean Florence flask and gently heat it ; it will presently be converted into a deep violet vapour, which, subliming to the cold parts of the vesisel, will there condense in small brilliant crystals, of an octoedral and rhomboidal figure. There is a substance called Naphthalin, obtained during the distillation of coal tar, and may commonly be procured at gas works : when pure, it is white and crystalline, and 
if heated in a large phial or flask, its vapour congeals in large lamellar crystals of extreme tenuity, crossing each other in all directions, and exhibiting a very singular appearance. When flakes of snow are examined, they are found to be a congeries of crystals of ice produced by the cooling of the vapour of water.

These are the principal means of obtaining bodies in the crystalline form, other methods being occasionally adopted in particular and difficult cases, some of which will be afterwards pointed out. Independent of the beautiful application of the process of crystallization in refined chemistry to determine the nature of bodies by the careful inspection of their forms, the manufacturer avails himself of it to cleanse, purify, and prepare a number of substances useful in the arts, the purity of which is often judged of by the regularity and perfection of their crystalline figures. Common salt is thus obtained by the evaporation of sea water and of brine springs, in consequence of its tendency to crystallize in hot liquors, which retain the other salts that are present in more permanent solution. Epsom salt, or sulphate of magnesia, is freed by crystallization from the muriate of magnesia with which it is mixed in the residue of sea water, after its common salt has been taken out: the muriate is very difficultly crystallizable, and remains in what is called the mother liquor, or residuary solution, which, on account of its excessive bitter taste, is known in our salt works under the name of bittern. Nitre is refined, by the facility of its crystallization, for the manufacture of gunpowder, and so cleansed of common 
salt, and a variety of other impurities, which render it perfectly unfit for that important purpose in its crude or native state, as generally imported from India. These are only a very few of the purposes to which the process of crystallization is applicable; many more will appear in the sequel.

A careful examination of the texture of crystalline bodies develops some curious facts respecting their structure and formation, which have attracted much attention among mineralogists. It has long been known to the mechanics employed in cutting and polishing the precious stones, that they yield new surfaces, and split, or separate, much more easily in one direction than another. This is especially remarkable in the diamond; and much of the skill of the artists employed in shaping that hard and beautiful gem for the market, consists in their acquaintance with its laminated texture, and their experience as to the direction in which the rough crystals will admit the formation of new surfaces. - But a simpler and easier illustration of the same fact is derived from the examination of crystals of calcareous spar, or carbonate of lime. This substance presents some hundred varieties of natural crystalline forms; but if we select any crystal, such, for instance, as the common variety called dog-tooth spar, and gently tap its different sides with a small hammer, we shall find that it yields, with singular readiness, in certain directions; that it splits into layers, which may be easily broken down into small rhomboidal fragments, of the annexed form.

Whatever may have been the original shape of the crystal, mechanical means enable us to dissect 
it into the above rhomboids; but these admit of no further change of form, the small ones being divisible into still smaller, but not into any other figure.

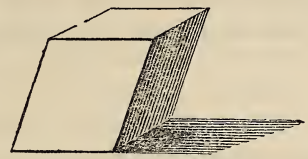

A rhomboid, therefore, of definite dimension or figure, is called the primitive form of calcareous spar; and it has been assumed that all crystalline substances are similarly divisible, each including a geometrical solid of peculiar figure. The primitive form of fluor or Derbyshire spar is a four-sided solid or tetruedron; and an eight-sided solid or octoedron, that of the diamond. But it is not difficult to conceive that these primitive forms may themselves be produced by certain arrangements of spherical or globular particles: thus, four balls,

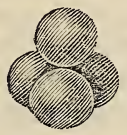

thus arranged, give the element of the tetraedral form; and six, arranged thus,

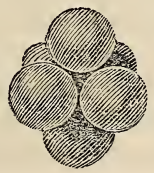


that of the octoedron, and so on. Instead, therefore, of assuming several distinct geometrical solids as primitive forms, we may refer to the sphere, or spheroid, as the source of all, and assume it as the figure of the ultimate mechanical particles of matter.

\$ 3.-Influence of Attraction upon the Chemical Composition of Substances.

The preceding paragraphs comprise an outline of the most important details respecting the influence of attraction upon the forms of matter, as far as that very extensive subject bears upon the inquiries of the chemist. We are now to proceed to a more exclusive subject of chemical investigation, and to state in general terms some of the principal results arising out of the influence of attraction upon the composition of matter. In the former case, attraction is said to act upon the homogeneous or similar particles of matter: in the latter, it operates upon heterogeneous or dissimilar particles, and is more commonly called Chemical Attraction or Affinity.

The most remarkable result of chemical attraction is the production of forms of matter possessed of properties entirely distinct from those of their component parts, so that it is impossible to anticipate, from our knowledge of the characters of the latter, those which are to belong to the resulting compound : hence chemistry is essentially a science of experiment. Oil of vitriol, for instance, or sulphuric acid, is a highly acrid and corrosive 
liquid, but it is composed of tasteless and comparatively inert elements, namely, oxygen, sulphur, and water. The elements, oxygen and nitrogen, which, in a state of mechanical mixture, constitute the air we breathe, give rise, as already stated, when chemically combined, to one of the most active agents of the laboratory; namely, nitric acid. Again, if sulphuric or nitric acids be combined with caustic potassa, comparatively mild and inert neutral salts result, namely, the sulphate and the nitrate of potassa, in which none of the properties of the component acid and alkali can be recognized. In art, these remarkable changes are made subservient to the most important purposes. A variety of extremely useful compounds are obtained by the union of elements comparatively useless and unimportant; and by the decomposition of apparently insignificant compounds, their more useful and important elements or components are separated. In nature, analogous changes are more strikingly presented to our observation, and the flower, the shrub, and the tree, are seen gradually to be formed and developed out of materials contributed by the air and soil, and in all respects most dissimilar to the new products of the vegetable: it is true that in these, and in analogous cases which might be taken from the animal kingdom, the mysterious power of vitality interposes; but such changes are nevertheless chemical, and exclusively objects of chemical inquiry.

There are many beautiful experiments illustrative of the total change of all obvious properties of substances as a consequence of chemical combination; as one striking instance, we may adduce 
the mutual action of oxygen and nitrous gases: these bodies are respectively permanently elastic fluids, without colour, taste, or smell : when mixed over water, they immediately enter into chemical combination, lose their elastic form, become of a deep orange colour, intensely sour, and of a peculiar odour ; in their separate state they are insoluble in water, but the body which has resulted from their combination is perfectly soluble in that fluid.

The evolution of heat and light are common consequences of chemical action, as exhibited in all cases of combustion. When a candle burns, the substances of which it is composed are entering into chemical combination with the oxygen of the surrounding atmosphere. So also with respect to the coals consumed in our fires; and in both cases we observe the combustibles gradually losing their solid form, and acquiring an invisible aëriform state; dissolving, as it were, in the air, and disappearing. If we apply a spark to a small heap of gunpowder, it is instantly dissipated in the gaseous form, and a bright flame ensues: in these cases it will always be remembered that there is no destruction of matter, but that the charcoal and other substances, composing the candle, the coal, and the gunpowder, have merely entered into new chemical arrangements, and are to be found, as we shall afterwards particularly show, in the products of combustion.

It has sometimes been imagined that the presence of air is requisite for combustion; and so, in fact, it is, in all the ordinary cases of that process; but there are abundant instances of combustion without its intervention. If we, for in- 
stance, heat sulphur with copper filings, the moment the metal has acquired a certain elevation of temperature, which is far below redness, it combines with the sulphur and becomes red-hot, forming, when cold, a black, brittle, and fusible compound, in which none of the properties, either of copper or of sulphur, are manifest: it is called sulphuret of copper. This experiment may serve to illustrate another important fact respecting chemical action, which is, that bodies combine in certain definite proportions only: for instance, the sulphuret of copper just adverted to, in whatever way it is formed, always contains 60 parts of copper combined with 16 of sulphur.

Among the general results of chemical combination, none are more striking than those which relate to the change of state or form sustained by the acting bodies. When substances are burned in the air, they generally pass from the solid to the aeriform state: the deflagration of gunpowder is a good instance of such sudden conversion; the converse change, that of gases into solids, is not so common, yet such cases are not unfrequent. When ammonia and muriatic acid, both gases, are mixed, they immediately condense into a white salt, which is muriate of ammonia; when a teaspoonful of fuming nitric acid is added to two of strong alcohol, the liquids presently disappear in the form of vapour; and when chlorine and carburetted hydrogen gases are mixed, they form a liquid of the appearance of oil : hence this variety of carburetted hydrogen has been called olefiant gas. These cases show the conversion of liquids into gases, and the converse. 
Liquids by combination produce solids, and solids liquids. Mix a strong solution of muriate of lime with one of carbonate of potassa, and they soon concrete when shaken together. Rub some crystals of sulphate of soda with an equal quantity of nitrate of ammonia, and the two salts gradually run down into a liquid.

Change of colour is a common, but also an important result of chemical action. By exposure to light and moisture, the greater number of colours are gradually destroyed, in consequence of the chemical agencies that ensue; a similar destruction of colour takes place more immediately upon the application of chlorine ; and hence these agents are resorted to, to effect the purpose of bleaching. In the arts of dyeing and calico-printing, not only are fugitive colours rendered permanent, but their tints are variously modified, by chemical means. Of the vegetable blues, there are many which are rendered red by an acid, and green by an alkali; and hence paper stained with the juice of violets, or of the blue flag, or of the petals of the blue heart's-ease, is useful as a test of the presence of those bodies. The same principle is applied to other purposes : if, for instance, an acid be gradually added to an alkaline solution, it is found that there are certain proportions in which they exactly neutralize each other; that is, a point at which the liquid neither exhibits acid nor alkaline characters. This point may be precisely learned by the above tests, for the solution then has no effect on the vegetable blue; but if either acid or alkali be predominant, that is, if the point of neutralization has not been exactly attained, then the 
test by the tint which it assumes announces the predominant element: green if alkaline, and red if acid.

Chemical action is promoted by whatever tends to disintegrate and separate the particles of matter, such as heat, solution, and mechanical division. Many substances which are without action at common temperatures, combine rapidly when sufficiently heated; thus a piece of charcoal is without action on the air till its temperature be elevated, when it gradually burns away and disappears, being converted into carbonic acid by uniting with the oxygen of the atmosphere. Dry crystals of citric acid and carbonate of soda exert no mutual action, as we see in what are called soda water powders; but when dissolved by water a violent effervescence shows the decomposition of the carbonate $:$ it is thus that we determine the presence of carbonic acid in minerals, salts, \&c., by pouring upon them a little dilute muriatic acid, when air-bubbles are very copiously evolved. There are many striking illustrations of the resistance afforded to chemical action by mechanical aggregation: the metal lead, in its ordinary state, is scarcely acted upon by the air at common temperatures ; but if the salt, called tartrate of lead, be heated red-hot in a small tube or phial, the black matter which is obtained contains the lead in a state of very minute mechanical division, and in this state it immediately takes fire upon exposure to air. In its ordinary state, platinum is entirely without action upon a mixture of hydrogen and oxygen gases; but in the porous and spongy form in which that metal is obtained by heating its ammonia-muriate, it instantly becomes red-hot when exposed to the mixed gases. 
Having noticed some of the leading consequences of chemical action, and of the circumstances which promote, retard, and modify its results, there are only a few other general remarks connected with the subject with which it is necessary that the reader should be thus early acquainted.

It is suficiently obvious that different bodies are possessed of different attractive powers; and accordingly, if several be brought together, those which have the strongest mutual attractions will enter first into union: hence it is that chemical attraction has also been called elective attraction. If I pour some dilute nitric acid upon a mixture of lime and magnesia, the acid will dissolve the lime in preference to the magnesia. The knowledge of this fact leads to the inference that lime would separate magnesia from its combination with nitric acid; and accordingly, if lime-water be added to a solution of nitrate of magnesia, nitrate of lime is formed, and magnesia is thrown down or precipitated. Such are the principles upon which the decomposition of bodies is effected, and which is sometimes a very simple, and at others a more complicated operation: for instance, sulphuric acid has a stronger attraction than nitric acid for baryta; if, accordingly, I dissolve a little baryta in nitric acid, and add to the solution a sufficient quantity of sulphuric acid, it will combine with the baryta, forming with it an insoluble precipitate, and, taking it entirely from the nitric acid, the latter will be left free. These changes are effected in the most direct way, and furnish an instance of what is called simple decomposition. But the nitric solution of baryta may also be decomposed by certain compounds 
containing sulphuric acid: thus, if I add a solution of sulphate of soda to one of nitrate of baryta, I shall, as in the former case, precipitate sulphate of baryta, and the soda previously in union with the sulphuric acid will be transferred to the nitric acid to form nitrate of soda: this is double decomposition, in which two new products are formed; and cases yet more intricate might be cited, furnishing instances of the varieties of complex decomposition.

One very important result of chemical attraction must not here be overlooked, which is, that bodies combine in certain proportions only. Water, for instance, is always constituted of one part of hydrogen and eight of oxygen. We have just adverted to the formation of sulphate of baryta, and have produced that compound by single and by double decomposition; sulphate of baryta may be formed by a variety of other processes, and it also exists native, being an abundant mineral product, found in many parts of the world: but in whatever way it is obtained, or whencesoever it comes, it is found that the sulphuric acid and baryta, which are its component or constituent parts, are always in the same relative proportions to each other; 78 parts of baryta being united in all cases to 40 of sulphuric acid, to constitute 118 of sulphate of baryta, or heavy spar.

There are numerous instances in which the same substances combine in several different proportions, producing as many distinct compounds, but these form no exception to the rule just laid down. Sulphuric acid, for instance, forms two distinct salts with potassa, called sulphate and 
bisulphate of potassa; in the former 48 parts of potassa are united to 40 of sulphuric acid, and in the latter to 80 , so that the one salt contains exactly twice as much sulphuric acid in combination with an equal quantity of potassa, as the former. These multiple proportions are yet more remarkably illustrated in those cases in which the same elements form a still greater number of compounds: thus nitrogen combines with oxygen in no less than five proportions, giving rise to as many distinct and definite compounds; in these 14 parts of nitrogen are respectively united to 8 , $16,24,32$, and 40 parts of oxygen, which latter numbers are, it will be observed, simple multiples, and to each other as $1,2,3,4$, and 5 : hence, in these and analogous cases, we say, that one proportional of nitrogen is combined with $1,2,3,4$, and 5 proportionals of oxygen*.

* These few facts, in reference to the definite proportions in which bodies combine, will, it is hoped, be sufficient to show the principle upon which chemists represent substances numerically, the numbers merely indicating the weight in which they unite; and it is obvious, that if we assume the lightest body in nature, which is hydrogen, as unity, the numbers representing other substances will be multiples of that unit; and it so happens, that whole numbers may be used to represent these multiples. Confining myself to instances which have been quoted in the preceding paragraph, the following table of equivalent numbers may be constructed.

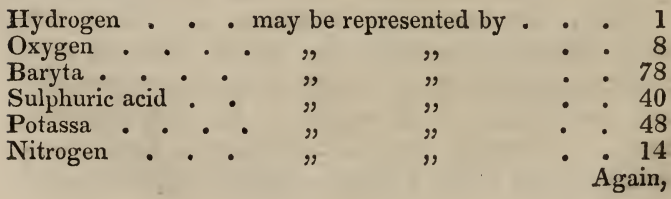


I shall not now proceed to apply this system of numerical representation to more complicated cases, wishing merely, at present, to show the principle upon which it is founded, and to exhibit it as a mere expression of facts, independent of theory or hypothesis. Some, however, observing the uniform relative weights in which bodies combine, have chosen to assume them as the representatives of their atoms; to say that an atom of hydrogen, weighing 1 , unites to an atom of oxygen weighing 8 , to form an atom of water weighing 9 , and so on : hence this subject is often discussed under the name of the atomic theory.

When the chemical nature of a body is to be determined, we endeavour to decompose or resolve it into its component parts, and to ascertain the nature of these, and the relative proportions which they bear to each other; this process is called analysis, and it often admits of verification by synthesis; that is, we are enabled to reproduce the original body by the reunion of its elements. This, however, is by no means always the case;

Again, in regard to some of the compounds which have been above enumerated,

Water composed of 1 hydrogen
and 8 oxygen

and 8 oxygen

Sulphate of baryta, containing 78$\}$

baryta and 40 sulphuric acid . $\}$

Sulphate of potassa, composed of 48 potassa and 40 sulphuric acid . . . . . . .

Bisulphate of potassa, consisting of 88 sulphate of potassa and 40 sulphuric acid, or of 48 potassa and 80 sulphuric acid

, 9

" $\quad$ " $\quad 88$


and, in many instances, especially in our researches on organic substances, we are obliged to rest content with analytical evidence. The term proximate analysis is often applied to the separation of two bodies which are themselves compounded, and ultimate analysis to the further separation of these compounds into their components. The composition of Epsom salt is synthetically demonstrated by uniting sulphuric acid to magnesia: analytically, by separating these proximate elements from each other. But sulphuric acid consists of oxygen and sulphur, and magnesia of oxygen and a metal called magnesium; consequently we should say, that the ultimate components of Epsom salt are oxygen, sulphur, and magnesium. 


\section{[ $\begin{array}{ll}33 & \text { ] }\end{array}$}

CHAPTER II.

HEAT-ITS EFFECTS AND SOURCES.

Heat may be considered as the antagonist power to attraction, one of its most obvious effects being the dilatation or expansion of bodies; so that, whenever a body is heated, it is enlarged in bulk, and, consequently, diminished in specific gravity. These changes are temporary only; for, on cooling, the body regains its former dimensions, and a further reduction of heat causes a proportionate shrinking or diminution of bulk: hence, bodies are said to expand when heated, and to contract when cooled - a law to which the exceptions are very limited.

We know nothing of the cause of heat, but we generally refer the effects perceived to the presence of a highly attenuated form of matter, which is supposed to pervade all substances, and to be diffused over the whole universe, and to which the French nomenclaturists gave the name of caloric -a term which has been much employed under the idea that the word heat, which is applied both to cause and effect, might lead to ambiguity: this, however, it need not; and whatever may be the cause of the phenomena, the word heat may be used to denote that state or condition of a body which excites in us the sensation of heat. 


\section{$\S 1$ - Of Expansion in general.}

The effects of heat are extremely varied and important; but those only which concern the chemist will here be chiefly dwelt upon: and of these, expansion may be considered as the most general and obvious.

Bodies exist in nature under three forms-solids, such as metal, \&c. ; liquids, as water; and gases or vapours, as oxygen gas and steam; these three forms of matter suffer expansion and contraction in very different degrees, when exposed to similar changes of temperature : in solids the change of dimensions is comparatively small, and refined means are necessary to determine and measure it; in liquids it is more obvious; and in gases extremely apparent.

To show in a rough way the expansion of a solid, a bar of metal, for instance, we may be provided with a gauge which measures its length and breadth at ordinary temperatures ; and it will be found that, when heated, it will no longer pass through the apertures which readily admitted it when cold.

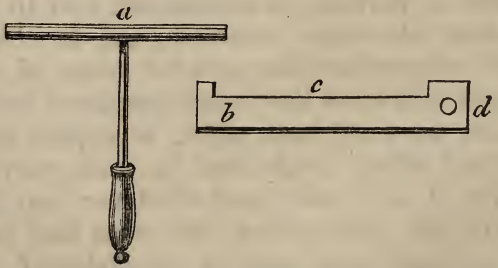

$a$ is a brass bar with a handle attached, the 
length of which at common temperatures is exactly equal to that of the notch $c$ in the plate $b$, and it exactly passes into the circular aperture $d$. If the bar be now heated by plunging it into boiling water, it will be found so much enlarged in all its dimensions, as not to pass through the apertures. If, on the contrary, it be cooled by ice, it will then contract, and pass them more readily.

The expansion and contraction of a liquid, when heated, are easily shown as follows :- pour into a large thermometer tube a quantity of brandy, so as

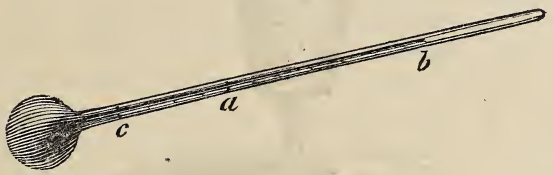

to fill the bulb, and rise in the stem up to the mark a. Plunge it into warm water, arid the spirit will rapidly dilate and rise in the tube, up to the point $b$; remove it, and suffer it to regain its original temperature, and its bulk will return to $a$; but if it now be put into ice, the liquid will be seen to contract, falling in the tube to $c$, for instance; but again expanding, when removed into the atmosphere at ordinary temperatures.

Aëriform bodies, or vapours, far exceed the former in expansibility. Place an empty thermometer tube, similar to that used in the last experiment, with its open end in a glass of water, as in the following wood-cut, and then apply the heat 


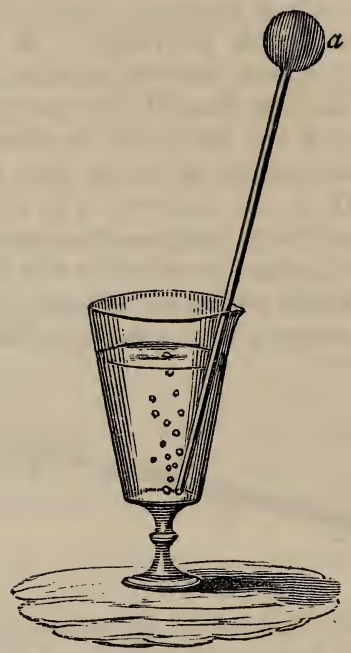

of the hand to the bulb $a$; this will be sufficient to expand the air within, so that several bubbles will escape through the water, and on removing the source of heat, the water will mount in the tube, showing, by the place it occupies, the amount of expansion which the air had suffered.

Thus far the general fact of the expansion of the different forms of matter by heat, and their contraction by cold, is easily demonstrated; but there are peculiarities belonging to each, and practical and useful applications resulting from them, which must be entered into more in detail. 
\$2.-Of the Expansion of Solids.

In the first place, in regard to solids, it is found that different bodies possess very different degrees of expansibility, and that among them the metals are most susceptible of change of bulk; but each metal is thus peculiarly affected, and possesses a rate of expansion of its own. In consequence of the numerous purposes to which the common metals are applied, and with which these calorific changes of bulk greatly interfere, it becomes necessary to examine their extent and consequences in these cases with minute precision. For this purpose bars of the respective metals have been very accurately measured, and their lengths carefully determined at the freezing point of water: the temperature of these bars has then been raised to the boiling point of water, and their increased length ascertained. The following is a table of results. The average expansion of glass is nearly the same as that of platinum.

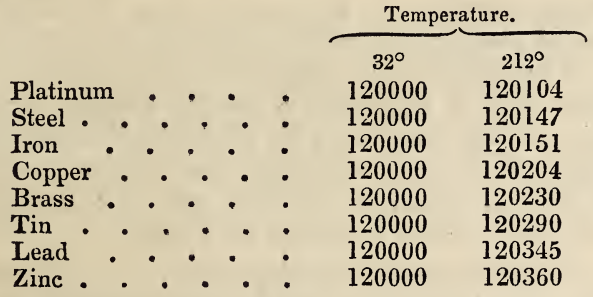

In consequence of the extensive use which is now made of iron in the construction of bridges, and as a substitute for timber in various parts of 
buildings, mechanics have become fully aware of the necessity of making due allowance for its expansion and contraction, and such arrangements, as may admit of it without injury to the other parts of the building. The arches of the Southwark Bridge, erected over the Thames by the late Mr. Rennie, rise and fall about one inch within the usual range of atmospheric temperatures. Where iron beams are employed, they must be so placed as to admit of some play without injury to the walls; for where such a beam has been incautiously placed, its contractions and expansions have often tended to endanger the whole building. Iron roofs, in consequence of their situations exposing them to the influence of a hot sun, or to the cold of winter, require peculiar care in this respect; and in other cases, apparently of trivial importance, very serious consequences have resulted from the incautious application of iron bars in situations where their changes of bulk could be productive of mischief, as in clamping stones together in the construction of steeples, and in placing iron railings between columns, \&c.

It has been above stated, that the expansion of glass and of platinum is nearly similar: hence wires of that metal may be welded "into fused glass without inconvenience; but if we substitute a wire of another metal, its different rate of contraction will tend to break the glass as it cools:

By a reference to the above table; it will be seen that iron or steel is much less expansible than brass; if, therefore, we form a compound bar of these or any other two differently expansible metals, by riveting or soldering them together, such a bar, 
by changes of temperature, will constantly warp in one direction or other, according to the relative position of the steel and brass. Let fig. 1 repre-

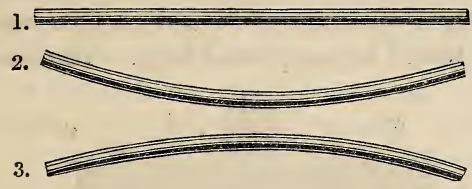

sent the compound bar, the steel being uppermost; if we then put it upon a heated plate, the bar will warp as shown in fig. 2, in consequence of the greater expansion of the brass : if, on the contrary, the compound bar be subjected to cold, it will assume the opposite curve, as shown in fig. 3, in consequence of the greater contraction of the brass. A compound plate of silver and platinum is remarkably susceptible of these contortions; and such contrivances have been applied to the construction of instruments for measuring changes of temperature.

The expansion and contraction of the metals by heat and cold is a serious inconvenience to the chronometer-maker, as necessarily interfering with the rate of going of the clock or watch. The pendulum, to vibrate seconds, must always be of a given length*, and it is obvious that if of metal it will be liable to shorten in winter and lengthen in

* The length of the pendulum vibrating seconds in vacuo in the latitude of London ( $51^{\circ} 31^{\prime} 8^{\prime \prime}$ North) at the level of the sea, and at the temperature of $62^{\circ}$, is $=39.13929$ inches of Sir George Shuckburgh's standard scale.-Kater, Phil. Trans. 1819, p. 415. 
summer; thus the clock will be fast in the former and slow in the latter season. The contrivances which have been resorted to, to counteract this inconvenience, are numerous, and many of them extremely ingenious; but any details upon the subject would be misplaced here. The following

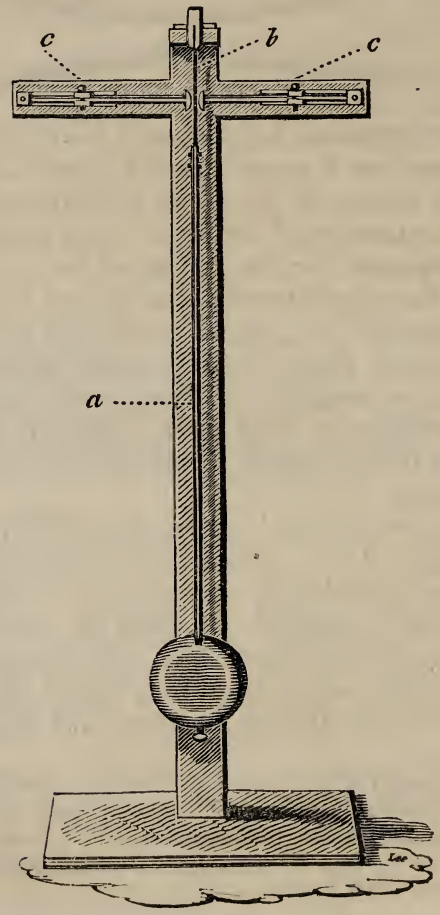


mode of compensation is, however, particularly illustrated by the principle explained above. $a$ represents the pendulum-rod of brass or steel, suspended by the thin plate $b$ between the compensation bars $c c$, which are firmly fixed at the ends $c$, and are supplied with proper adjustments, which it is unnecessary here to describe. Let us now suppose the pendulum exactly vibrating seconds at the temperature of $62^{\circ}$, and that the temperature rises to $80^{\circ}$ : in this case, the bar $a$ would be proportionately elongated, and it would not then vibrate so many as 60 seconds in the minute; but the effect of increase of temperature in the compensation bars, supposing the brass uppermost, would be to warp them, as in the following sketch, ( $f g .1$,$) and, consequently, to shorten$
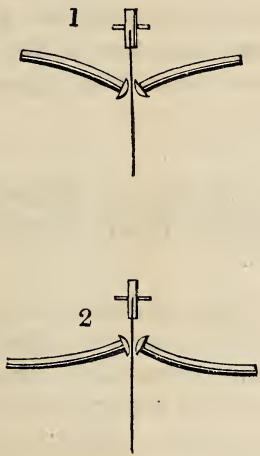

the length of the pendulum ; and in cold weather, on the contrary, the rod of the pendulum would be 
shortened, but the bars warping upwards would tend to elongate it as in fig. 2. Exactly a similar plan of compensation may be applied to the balance-springs of watches, which are affected in the same way, and with the same effects, as the pendulum of a clock.

The dimensions of wood are much less altered by changes of temperature than those of metals; it is, therefore, occasionally used for the rods of very large pendulums; but, in other cases, it is not found effectual, and is liable to be deranged by the varying moisture of the atmosphere.

There are some mechanical operations, in which the expansion and contraction of metals by heat and cold are very beneficially applicable: by putting the hoops upon a cask in a heated state, they gradually contract on cooling, and firmly bind the staves together; and, with the same view, the wheelwright heats the tire of his wheel, that it may firmly press upon the circumference as it cools.

3.-Experiment showing the Expansion of LiquidsAnomaly in regard to Water-Production of Currents, and their Effects.

The expansibility of different liquids, like that of solids, is liable to much variation: ether, for instance, is more expansible than alcohol, and alcohol more so than water, and water than mercury. Liquids are also differently expansible at different temperatures; and, generally speaking, their rate of expansion increases with their temperature. This irregularity is chiefly observed in those 
liquids which readily boil: where they require a very high temperature to convert them into vapour, it is less observable. The expansion of mercury, for instance, at low and high temperatures, is only slightly dissimilar; hence, as we shall presently find, one of the great advantages of that, over other liquids, in the construction of thermometers.

There is a very remarkable exception to the law of expansion and contraction by heat and cold, in the case of wäter, which, unlike all other fluids, expands when cooled below a certain point. All other fluids go on diminishing in bulk till they freeze; but if we fill a large thermometer tube with water, of the temperature say of $80^{\circ}$, and then plunge the bulb into a freezing mixture of pounded ice and salt, we shall observe the following curious appearances.-The water will go on shrinking in the tube, till it has attained the temperature of about $40^{\circ}$; and then, instead of continuing to contract till it freezes, (as is the case with all other liquids, it will be seen slowly to expand, and consequently to rise in the tube until it congeals. In this case the expansion below $40^{\circ}$ and above $40^{\circ}$ seem to be equal, so that water will be of the same bulk at $32^{\circ}$ as at $48^{\circ}$, that is, at $8^{\circ}$ above or below $40^{\circ}$.

This anomalous expansion of water by cold is not merely a curious philosophical fact, but it is productive of some most important and beneficial consequences in nature; for, let us suppose that water, like other fluids, went on diminishing in bulk, or, in other words, increasing in density, till it froze; the consequence would be, that large bodies of water, instead of being only superfi- 
cially frozen in winter, would be converted into solid masses of ice. Let us take a fresh-water lake as an example. The earth being warmer in winter than the air, the heat is withdrawn from the surface of the water by the cold breezes that blow over it, and the whole body of water has its temperature lowered to $40^{\circ}$, which is the point of its greatest density, and a temperature perfectly congenial to fish and most other aqueous animals. The cold now continues to operate upon the surface of the water, but instead of diminishing its bulk, and rendering it heavier than the warmer water beneath, it expands and renders it lighter; so that, under these circumstances, a stratum of ice-cold water (at $32^{\circ}$ ) will be found lying upon a mass of warmer water beneath it (at $40^{\circ}$ ). The influence of the cold continuing, the surface of the lake will soon freeze, but the water immediately below the superficial coating of ice will be found compara-

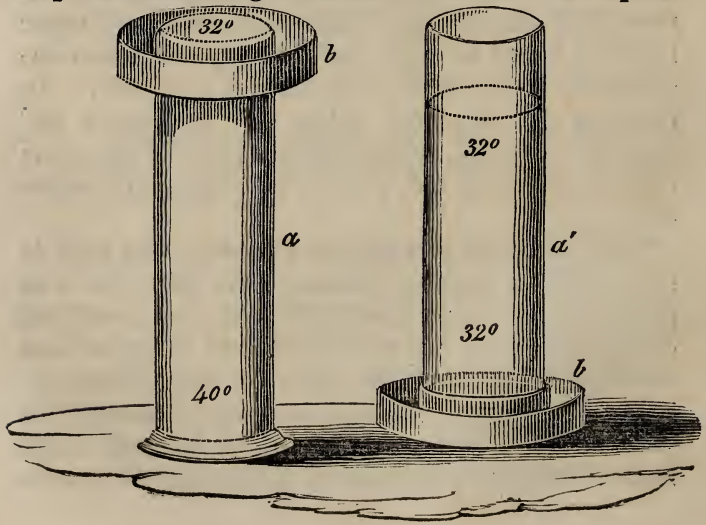


tively warm; and as water is almost a non-conductor of heat, it will be a long time before the ice attains any thickness; and the whole body of water, if of any depth, can never freeze throughout. Indeed, it will be obvious that the retardation of freezing will be proportional to the depth of water which has to be cooled, and hence some very deep basins or lakes are scarcely ever covered with ice.

Perhaps the following experiment may render this peculiar property of water more intelligible. Let $a a^{\prime}$, in the preceding woodcut, represent two cylindrical glass vessels, filled with water at $60^{\circ} ; b b^{\prime}$ are tin trays surrounding and closely fitting the exteriors of the cylinders, and intended to contain a freezing mixture of snow and salt, which in $a$ is applied to the upper surface, but in $a^{\prime}$ to the bottom of the water. The first effect of the cold will be to reduce the temperature of the whole bulk of water, in both vessels, to $40^{\circ}$, that is, to the point of greatest density. After which it will be found that, in the vessel $a$, the cooling effect will not proceed downwards, but will be limited to the surface, where the thermometer will gradually fall to $32^{\circ}$, and the water probably freeze; for the ice-cold water being lighter than the water at $40^{\circ}$ below, it will necessarily float upon the surface: a thermometer, therefore, in the upper part of the vessel will fall to $32^{\circ}$, but in the lower part only to $40^{\circ}$. This may be said to represent the case of the lake above described. In the second cylinder $a^{\prime}$, the cold, instead of being applied to the surface of the water, is applied to the bottom, and the effect is, of course, very different; for now 
the cooled water, becoming lighter, ascends, whilst the warmer water descends, and becoming cooler in its turn, the whole body of water is reduced to the freezing temperature-an effect limited to the surface in the other cylinder, which represents what happens in nature, where the cold is always superficial; and ice being lighter than water, it is; of course, formed, and remains upon the surface.

As liquids are enlarged, and consequently rendered specifically lighter, by heat, it follows that, in heating a mass of liquid, very different effects will be produced by applying heat to different parts of

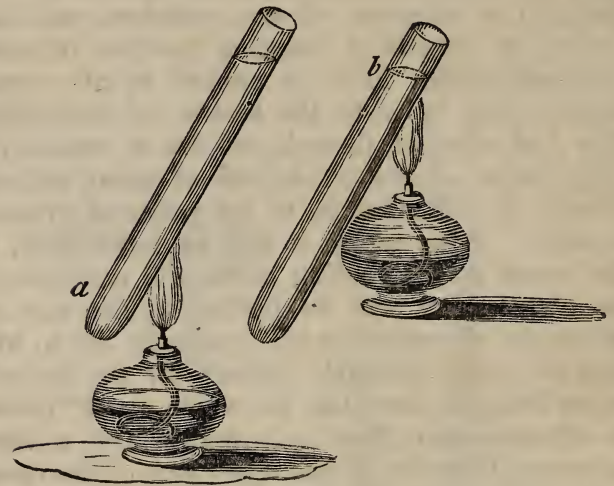

the vessel containing it: $a$ and $b$ represent two tubes of thin glass filled with water. If we apply the flame of the spirit-lamp to the bottom of the tube $a$, the water will soon be heated equally throughout, and boil ; and this is the usual way of apply- 
ing heat, namely, to the bottom of the boiler or saucepan; but if, as in the tube $b$, we heat the surface of the water, we shall find that it may be made to boil in the upper part of the tube, whilst the water at bottom will remain cold. In this case, the water becomes lighter by being heated, and consequently floats upon the colder water below, to which scarcely any heat will be communicated.

The currents that ensue, in the ordinary way of heating water, may be rendered obvious by the following experiment :- Fill a cylindrical glass vessel, of the shape annexed, with water, and throw into it a few particles of bruised amber, or any other convenient substance, nearly of the same specific gravity as the water, so that, if possible, they may neither sink nor float in it; then apply a gentle heat to the bottom of the vessel, and it will be seen that currents immediately begin to ascend in the centre, or axis, and to descend towards the circumference of the vessel, in the directions shown by the darts. These currents soon become rapid in their motions, and continue till the water boils. It is, therefore by the changes in the weight of the different portions of water, that we succeed in conveying heat throughout the mass. The heated portion becomes enlarged in bulk, and, therefore, specifically lighter than the adjacent cold portions through which it ascends; whilst they, descending, come into the contact of the heated surface, and in their turn expand and rise. This goes on till the whole of the water has acquired its boiling temperature, beyond which it cannot be heated, and therefore the currents then cease. 


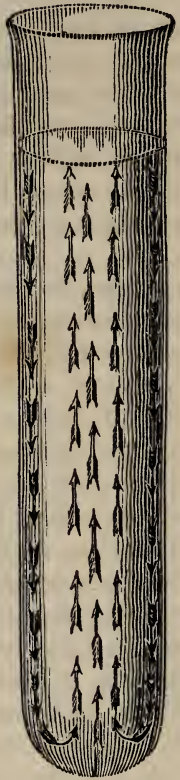

Such currents may be established, in properlyconstructed vessels, to any extent, and heat thus communicated to water at a very considerable distance from the boiler; a system lately adopted very advantageously in hot-houses, conservatories, and manufactories, and for heating baths. The contrivances for these purposes are very various; but all, of course, imply the necessity of an ascending and descending current. 
\$ 4.-Expansion of Aëriform Bodies-Currents in the Air -Theory and Practice of Ventilation.

We may now notice the effects of heat upon gases, air, and vapours. These, as we have stated, undergo much greater relative changes of bulk than the other forms of matter, but they also present us, in this respect, with an important peculiarity. In other substances, we observe that each individual has its own degree of expansion and contraction, whereas all pure aëriform bodies expand and contract alike : so that, if we accurately determine the expansion and contraction of any one of them, in a given range of temperature, that knowledge applies to all the rest; and it is a very important object, to the experimental philosopher, accurately to determine these changes of bulk. It is found that 100 measures of air, when heated from the freezing to the boiling point of water, suffer an increase in bulk equal to $\mathbf{3 7 . 5}$ parts; so that 100 cubic feet of air, at $32^{\circ}$, become dilated to $137 . \frac{1}{2}$ cubic feet at $212^{\circ}$. Steam, and all other vapours, when heated out of contact of their respective fluids, are subject to laws of expansion similar to those of air. The history, therefore, of aëriform bodies, in respect to this effect of heat, is extremely simple.

The dilatation suffered by air, when heated, being very considerable, is, of course, attended by a proportionate diminution in the specific gravity or relative weight of the heated portion, which rapidly ascends through the colder mass, and excites powerful currents and agitations in 
the general bulk of air. These currents often escape our observation, in consequence of the invisibility. of air; but they may easily be rendered evident; and often excite our attention, by their violence-as when powerful draughts, wind, and so forth, are produced.

In consequence of the lightness of heated air, it always rises to the upper parts of rooms and buildings, when it either escapes, or, becoming cooled and heavier, again descends. If, in cold weather, we sit under a skylight in a warm room, a current of cold air is felt descending upon the head, whilst warmer currents, rising from our bodies and coming into contact with the cold glass, impart to it their excess of heat. Being thus contracted in bulk, and rendered specifically heavier, they in their turn descend, and thus a perpetual motion is kept up in the mass of air. This effect is attended with much inconvenience to those who inhabit the room, and is in great measure prevented by the use of double windows, which prevent the rapid cooling and production of troublesome currents in the air of the apartment.

We generally observe, when the door of a room is opened, that there are two distinct currents in the aperture ; which may be rendered evident by holding in it the flame of a candle. At the upper part it is blown outwards, but inwards at the lower part; in the middle, scarcely any draught of air, one way or other, is perceptible.

The art of ventilating rooms and buildings is in a great measure dependent upon the currents which we are enabled to produce in air by changes of temperature, and is a subject of consider- 
able importance. As the heated air and effluvia of crowded rooms pass upwards, it is common to leave apertures in or near the ceiling for their escape. Were it not, indeed, for such contrivances, the upper parts of theatres and of some other buildings would scarcely be endurable ; but a mere aperture, though it allows the foul air to escape, in consequence of its specific lightness, is also apt to admit a counter-current of denser and cold air, which pours down into the room, and produces great inconvenience. This effect is prevented by heating, in any convenient way, the tube or flue through which the foul air escapes. A constantly ascending current is then established; and whenever cold air attempts to descend, the heat of the flue rarefies and drives it upwards. Thus the different ventilators may terminate in tubes connected with a chimney; or they may unite into a common trunk, which may pass over a furnace purposely for heating it.

In some of our theatres, the gas chandelier is made a very effectual ventilator. It is suspended under a large funnel, which terminates in a cowl outside the roof; and the number of burners heat the air considerably, and cause its very rapid and constant ascent through the funnel, connected with which there may be other apertures in the ceiling of the building. But in these and most other cases, we may observe that the vents are not sufficiently capacious; and the foul air from the house, and from the gas-burners themselves, not being able readily to escape, diffuses itself over the upper part of the building, and renders the galleries hot and suffocating-all which is very easily prevented 
by the judicious adjustment of the size of the ventilating channels to the quantity of air which it is requisite should freely pass through them.

The small tin ventilators, consisting of a rotating wheel, which we sometimes see in window-panes, are perfectly useless, though it is often imagined, in consequence of their apparent activity, that they must be very effectual ; but the fact is, that a very trifling current of air suffices to put them in motion, and the apertures for its escape are so small as to produce no effectual change in the air of the apartment: they are also as often in motion by the ingress as by the egress of air.

From what has been said, it will be obvious that our common fires and chimnies are most powerful ventilators, though their good services in this respect are often overlooked. As soon as the fire is lighted, a rapid ascending current of air is established in the chimney, and consequently there must be a constant ingress of fresh air to supply this demand, which generally enters the room through the crevices of the doors and windows, When these are too tight, the chimney smokes or the fire will not draw; and in such cases it is sometimes necessary to make a concealed aperture in some convenient part of the room for the requisite admission of air, or to submit to sitting with a window or door partly open. - Any imperfect action of the chimney, or descending current, is announced by the escape of smoke into the room, and is frequently caused by the flue being too. large, or not sufficiently perpendicular and regular in its construction. When there is no fire, the chimnies also generally act as ventilators; and in 
summer there is often a very powerful current up them, in consequence of the roof and chimneypots being heated by the sun, and thus accelerating the ascent of the air. In a well-constructed house there should be sufficient apertures for the admission of the requisite quantity of air into the respective rooms, without having occasion to trust to its accidental ingress through every crack and crevice that will allow it to pass. These openings may either be concealed, or made ornamental, and by proper management may be subservient to the admission of warm air in winter.

The manner in which heat is distributed through our apartments by the fires in common use will be noticed afterwards; but large rooms are not uncommonly warmed by heated air, admitted usually through apertures in the floor. This system is extremely convenient, effective, and agreeable, when properly managed, but this is seldom the case. The rooms are close and oppressive, because due ventilation is not associated with the admission of hot air; the apertures by which the air is admitted are generally too small, and the air itself overheated: the openings should be large, and the quantity of air thrown in should be considerable, but its temperature moderate. In this way the bad smell produced by burnt particles of dust is avoided, and an agreeable freshness as well as temperature kept up in the air. 
\$ 5.-Of the Construction and Uses of Thermometers and Pyrometers, and of the Scales applied to them.

Having thus stated some of the general effects of contraction and expansion in the different forms of matter, we may now proceed more particularly to the examination of the thermometer-an instrument of considerable importance in all our researches connected with the subject of heat.

The first instrument to which the term thermometer can properly be applied, was apparently invented by the Florentine Academicians, about the middle of the seventeenth century. It consisted of a glass tube, with a bulb or ball blown at one extremity, and filled, up to a certain mark, with spirit of wine; the expansion of which, or its rise above the mark, indicated heat, and its contraction, or fall below the mark, cold. They afterwards placed a number of little equidistant studs of white enamel on the tube, rendering the expansions and contractions of the confined liquid more evident; and by these they sometimes divided it into a hundred parts or degrees. Such instruments were first introduced into England by Boyle. For a long time, no one would believe that a liquid could contract and expand in a tube closed at both ends; but Boyle soon satisfied himself of the fact by experiment, 
and by the inspection of the Florentine glasses, for so these instruments were then called.

About the beginning of the eighteenth century, a considerable improvement was made in this original thermometer, by Fahrenheit, of Amsterdam, who, by the use of mercury instead of alcohol, was enabled greatly to diminish the size of the tube and bulb, and to measure comparatively high degrees of temperature.

But all these instruments laboured under the serious defect of entire want of agreement with each other: the division of the tube was founded on no fixed principle, but was arbitrary, and differently constructed and applied in every thermometer. It required no common ingenuity to overcome this difficulty, and to construct instruments which should agree with each other when exposed to the same temperature. This, however, was at length effected by a suggestion of Sir Isaac Newton, founded upon a discovery of Dr. Hooke, who observed, that when a thermometer tube, properly filled with mercury, was plunged into melting ice or snow, the liquid metal always contracted, or fell to a certain point, at which it remained perfectly stationary till all the ice was melted. If we now mark this point upon the tube, it will be found that, whenever it is subjected to the same experiment, the quicksilver falls exactly to the same level or point, and hence this point may be called the melting-point of ice; and it has since been ascertained that this is also precisely the point at which water, under ordinary circumstances, freezes, so that it is also called the freezing-point of water. Here, then, a part at 
least of the difficulty above adverted to is removed, and one fixed point for thermometric graduation ascertained.

Dr. Hooke afterwards made a similar discovery in regard to boiling water, which, under ordinary circumstances, he found always exhibited one and the same degree of heat. If, therefore, we now plunge our thermometer, upon which the freezing-point of water has already been marked, into an open vessel of water placed upon the fire or over a lamp, we shall observe the mercury continue to rise in the tube until the water boils, when it becomes quite stationary, and the point may be marked as before. Now it will be found, that whenever this thermometer is put into boiling water, the mercury will rise to the same height*. Here, then, we have a second determinate point, namely, the boiling-point of water; and it is now obvious, that if we divide the space of the tube between these two fixed points into any given number of degrees, the mercury will indicate the same degree in all bodies of one and the same temperature; and that any number of thermometers, carefully constructed upon this principle, will agree with each other in their indications of temperature. The space between the boiling and freezing of water has often been divided into one hundred equal parts - the freezingpoint being marked $0^{\circ}$, and the boiling-point $100^{\circ}$.

* Some circumstances slightly affect the boiling-point, such as the state of the barometer or of the pressure of the atmosphere, and the nature of the vessel in which the experiment is made. We presume above that the barometer stands at thirty inches, and that the vessel is of thin metal. 
This is commonly called the centigrade scale, and is used in France and many other parts of Europe. Réaumur's scale calls the freezing-point $0^{\circ}$, and the boiling-point $80^{\circ}$; but in this country we generally refer to Fahrenheit's scale, which sets out at the temperature produced by mixing snow with salt, and which was erroneously conceived to be the lowest artificial temperature. It is, in fact, $32^{\circ}$ below the freezing of water, and the space between that and the boiling-point being divided into $180^{\circ}$, the latter is called $212^{\circ}$. In all these scales the graduation is continued downwards below $0^{\circ}$, in the same way as above it. These are called degrees below $0^{\circ}$, and are generally distinguished by prefixing the mark (-) minus before them: thus -10 means ten degrees below $0^{\circ}$, and sometimes the mark $(+)$ plus is prefixed to the degrees above zero, for the sake of greater precision; as +10 , or ten degrees above $0^{\circ}$.

Such are the principles upon which thermometers and their scales are constructed, and for all common purposes ordinary thermometers are sufficiently accurate ; but the experimental laboratory should be furnished with a standard thermometer, very carefully constructed, and with a scale exhibiting the divisions of Réaumur and Fahrenheit, and the centigrade. Such an instrument of reference is useful to determine the quality of common thermometers, no two of which will be found precisely to agree at the different points of the scale.

The thermometer having advanced thus far, it became an important question to determine how far its degrees actually represented equal quantities or increments of heat; and an ingenious experi- 
ment, originally contrived by Dr. Brook Taylor, seems to show that this really is the case. It consisted in mixing together, with due precaution, equal parts of hot and cold water ; and it was found that the thermometer, applied to such a mixture, indicated the arithmetical mean between the extremes. Thus, if we mix a pound of water at the temperature of $200^{\circ}$ with a pound at the temperature of $100^{\circ}$, an accurate thermometer will show in the mixture the mean temperature of $150^{\circ}$.

The indications of the mercurial thermometer are obviously limited within the freezing and boiling points of mercury. It congeals at $40^{\circ}$. below $0^{\circ}$, and boils at $650^{\circ}$; and on the moment of its congelation it shrinks so remarkably, as to have led early experimentalists into considerable errors as to the actual cold required for the purpose. When it boils, its vapour, of course, bursts the tube. Although, therefore, the range of temperature which may be accurately measured by the expansion and contraction of mercury is very extensive, it is often requisite to measure degrees of cold below its freezing-point, and of heat above its boiling-point. Pure spirit of wine or alcohol has never been frozen, and it is consequently used in those thermometers which are intended to indicate excessive degrees of cold; but no fluid has yet been found applicable to the construction of a thermometer capable of indicating higher temperatures than mercury. When, therefore, a degree of heat approaching to, or exceeding dull redness, is to be measured, other methods and substances must be resorted to: instruments constructed for this purpose are called pyrometers.

It is needless here to describe Wedgwood's 
pyrometer, though it is often referred to in scientific works; for its indications are never to be depended on, and are frequently greatly in error. Mr. Daniell has contrived an instrument capable of measuring, with great nicety, the expansions of a bar of platinum at high temperatures, and has pointed out a method of comparing them with the degrees upon Fahrenheit's mercurial scale. The following points were thus determined:-

Degrees of Fahrenheit's Thermometer.

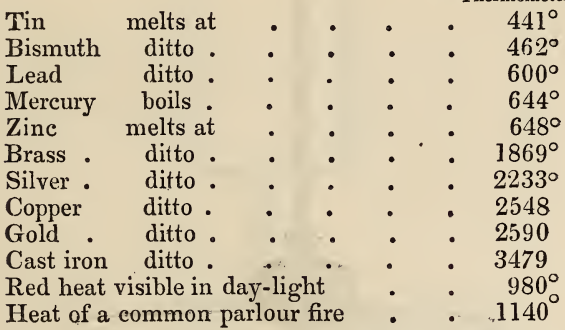

Several points are occasionally particularly recorded upon our thermometric scales, such as remarkably high and low natural temperatures, the boiling-point of alcohol, ether, \&cc., the heat of the human blood, the heat of springs and baths, and the degrees required for forcing various fruits. These, of course, refer to the particular purposes for which the thermometer is intended.

Sometimes the expansions and contractions of air, which, as already stated, are relatively great, for small changes of temperature, are resorted to in delicate experiments, in which slight variations of heat require to be indicated. 
The simplest form of the air thermometer consists of a tube with a thin bulb at its upper end, and dipping at the lower and open extremity into a

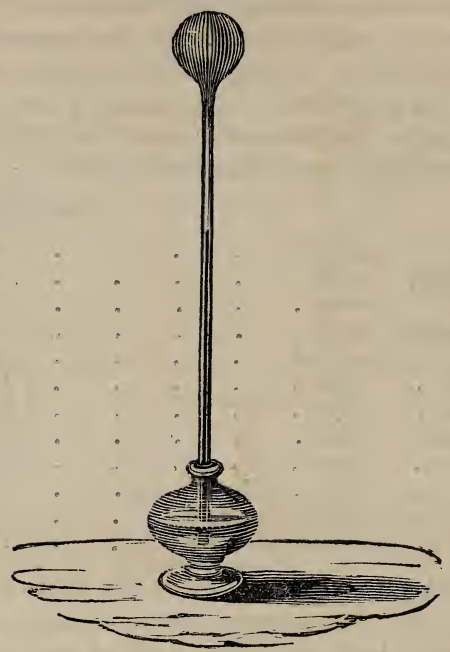

little coloured fluid ; some of the air is then expelled by the heat of the hand, so that, as the bulb cools, the liquid may rise to about half way up the tube. This instrument is very sensible to slight changes of temperature: heat expands the air in the bulb, and drives the fluid downwards ; and cold, by contracting the air, causes the fluid to rise. This was one of the first forms of the thermometer that was invented, and is only inconvenient from its extreme delicacy, and the great length of tube 
required to measure any considerable range of heat and cold.

Another and much more useful form of air thermometer is represented in the following woodcut: it has sometimes been called the differ-

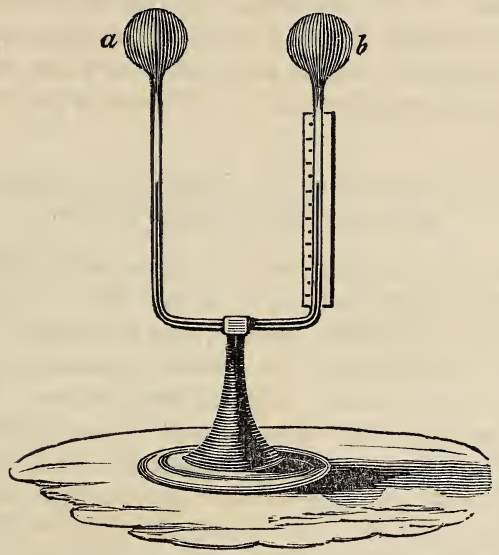

ential thermometer. It consists of two bulbs, $a$ and $b$, containing air united by a tube twice bent at right angles, in which there is a little coloured sulphuric acid: the tube is supported upon a wooden foot or stand. When a hot body is brought near one of the bulbs, it expands the air within it, and drives the fluid towards the other-from $b$, for instance, to $a$. One of the chief advantages of this air thermometer is, that it is not affected by any general changes in the temperature of the surrounding air, because they act equally on both 
bulbs, and occasion no motion of the included column of liquid ; but when one of the bulbs is exposed to any source of heat which does not affect the other, the difference of temperature between the two bulbs is immediately and very sensibly indicated by the recession of the fluid from the heated towards the cold bulb. The advantages of this instrument will be more evident when some of its practical applications are afterwards pointed out.

$\S 6 .-O f$ the relation of the Temperature of Bodies to the quantity of Heat contained in them.

That the thermometer is an accurate measure of temperature, has been shown by Dr. Taylor's experiment already quoted, but it affords us no correct insight into the absolute quantity of heat necessary to produce a given temperature: if, for instance, a pint of olive oil and a pint of water be brought from a cold into a warm room, from a temperature perhaps of $40^{\circ}$ to a temperature of $70^{\circ}$, they will, it is true, gradually acquire the same temperature; and thermometers immersed in the respective liquids will gradually rise from $40^{\circ}$ to $70^{\circ}$; but if we carefully watch the manner in which this rise of temperature is brought about, we shall find that the oil acquires the temperature of $70^{\circ}$ much more rapidly than the water; whence it may be inferred, that the oil requires less heat to raise it from $40^{\circ}$ to $r 0^{\circ}$ than the water. Again, if we reverse the experiment, and transfer the two liquids from a warm to a cold room, it will then be observed that the oil cools more rapidly than the water : the fact is, that the quantity of heat required 
to raise water from $40^{\circ}$ to $70^{\circ}$ is much greater than that required to raise the oil a similar number of thermometric degrees, and consequently oil is much more rapidly heated and cooled than a corresponding quantity of water. The following experiment proves this to be the case.-A pint of water at $212^{\circ}$ was surrounded, in a proper apparatus, by ice, and the quantity of ice which was thawed by the heat given out by the water in cooling from $212^{\circ}$ to $32^{\circ}$, was accurately ascertained. A similar experiment was then made with oil, and it was found that, in passing through the same range of temperature, it only thawed half as much ice as the water: although, therefore, the temperatures of the liquids were the same at the outset and conclusion of the experiment, the quantity of heat given out by the oil was only half that given out by the water; whence we infer that bodies, though of the same temperature, do not contain the same quantity of heat-some requiring more, and some less heat, to raise them to a given thermometric point. The quantity of heat thus required is called the specific heat of the body; and if, in reference to the above statement, we call the specific heat of water $100^{\circ}$, that of oil will only be $50^{\circ}$; or, in other words, water has twice the capacity for heat that oil has. These terms must, however, be carefully limited to bodies retaining one and the same state; for when they change their state, their relations to heat are singularly affected, as we shall soon find.

But although a body may not change its state or form, its capacity for heat will be found very materially affected by its density; and whenever density is diminished, capacity for heat is increased-and 
vice versa. Thus, in regard to solids, if we suddenly compress a piece of metal, it becomes hot, because its capacity for heat is diminished; and therefore, in its dense and compressed state, it contains less heat than it did before; this is seen in the process of coining, where the blank piece, in sustaining the sudden and violent pressure of the coining-press, becomes suddenly warm; and a piece of soft iron, upon being dexterously struck a few heavy blows with a hammer, becomes hot enough to inflame the brimstone of a match. A piece of Indian rubber, suddenly and forcibly drawn out, becomes warm in consequence of the extension, as may easily be perceived by applying it to the lip the moment it is stretched, that part being very susceptible of slight changes of temperature.

Similar changes of capacity for heat, attending change of density, are also perceived in liquids. When sulphuric acid is mixed with water, the relative densities of the mixture are suddenly and greatly increased, and it becomes boiling hot. When alcohol and water are mixed, there is also a diminution of density, and heat is evolved.

In gases we have a convenient opportunity of observing that, as on the one hand, condensation produces heat, so, on the other, rarefaction or diminution of density is attended by increased capacity for heat, or, in other words, by the production of cold. Air suddenly compressed by the piston of a syringe, evolves so much heat as to inflame combustibles exposed to it; but if we suspend a thermometer in the receiver of the air-pump, we shall find that, during exhaustion, the rarefaction or expansion which the 
air suffers, causes a diminution of several degrees of temperature; and this is the reason why the air in the higher regions is so much cooler than that below. As it ascends from the earth it expands ; and in expanding its capacity for heat is increased : it therefore cools itself, as well as the surrounding bodies.

The Andes, placed almost under the line, rise in the midst of burning sands ; about the middle height is a pleasant and mild climate; the summits are covered with unchanging snows; and these ranges of temperature are always distinct. The hot winds from below, if they ascend, become cooled in consequence of expansion; and the cold air, if by any force of the blast it is driven downwards, is condensed and rendered warmer as it descends.

When hot steam suddenly issues, under great pressure, from a small aperture into the atmosphere, its instantaneous expansion so far cools it, that, instead of scalding the hand held in it, as is the case with ordinary steam, it scarcely feels warm.

Another case in point is furnished by the Fountain of Hiero, as it is called, at Schemnitz, in Hungary. A part of the machinery for working these mines is a perpendicular column of water, 260 feet high, which presses upon a quantity of air inclosed in a tight reservoir : the air is consequently condensed to an enormous degree by this height of water, which is equal to between eight and nine atmospheres; and when a pipe communicating with this reservoir of condensed air is suddenly opened, it rushes out with extreme velocity, instantly expands, and in so doing it absorbs so much heat as to pre- 
cipitate the moisture it contains in a shower of very white compact snow, which may readily be gathered on a hat held in the blast. The force of this is so great, that the workman who holds the hat is obliged to lean his back against the wall, to retain it in its position.

If the blast from an air gun be directed upon a delicate thermometer, it sinks it a few degrees, from the cold produced by the sudden expansion of the air.

§ 7.-Solids, Liquids, and Gases, considered us Conductors of Heat.

Having described the construction, and some of the uses of the thermometer, and having shown that different bodies require different quantities of heat to raise them to the same temperature, we may now revert to a subject already in part discussed, namely, the propagation of heat through different bodies.

One method by which heat diffuses itself has been noticed above; for it has been shown that, in liquids and gases, heat produces expansion, and, in consequence of the mobility of their particles, makes its way, and is diffused through them, by change of specific gravity.

Heat is also propagated through bodies by their conducting powers. A metal bar is well calculated to illustrate this conduction of heat: if one end of it be held in the candle, and the other in the hand, the heat gradually and progressively makes its way from one end to the other; if the hand be near the source of heat, or the bar short, it soon becomes too hot to hold. If we employ bars 
of different metals, it will be found that some conduct heat better than others: thus, if two similar wires, one of platinum and one of silver, be heated at one end, the heat will pervade the silver much more rapidly than the platinum; and if cones of several metals, like the one represented in the following cut, be respectively tipped with a small piece

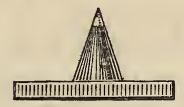

of wax, and placed upon a heated plate of metal, the wax will melt at different perioass upon the different cones, showing their relative conducting powers.

Silver, gold, copper, and tin, are the best conductors, and nearly equal; iron, platinum, and lead, are much inferior. It does not appear, therefore, that the conducting power corresponds either with the density, the tenacity, or the fusibility of the metals. Next to metals, the diamond, the topaz, and some earthy compounds, appear to have the greatest conducting power: then stony bodies, porcelain and glass, and porous earthy compounds, such as earthenware and bricks. Wood is a very bad conductor of heat; so is charcoal; and these may be held, without burning the fingers, very near the red-hot part.

The porous vegetable and animal substances used in clothing, and the natural coverings of most animals, are also imperfect conductors of heat; but in these cases interstitial air cannot be excluded, and consequently their true conducting power is not 
accurately determinable : however, Count Rumford found that when a heated thermometer, surrounded by about an inch in thickness of confined air, took 576 seconds to cool $135^{\circ}$, it took 1032 seconds to cool to the same point when 16 grains of lint were diffused through the confined air, and 1305 seconds when the same weight of eiderdown was used; so that, as the conducting power is inversely as the times, if the power of air be reckoned as 1000 , that of the lint will be 558 , and of the eiderdown $44 \mathrm{l}$. The conducting power of these substances is further diminished by the closeness with which their particles are compressed, provided, that is, their texture is not too closely compacted: thus, when the same space which in the above experiment was occupied by 16 grains of eiderdown, was filled first with 32 grains, and then with 64 , the times employed in the escape of the 60 degrees of heat from the enveloped thermometer were successively increased from 1305 seconds to 1472 and 1615 seconds.

To show the influence of mere texture upon conducting power, similar trials were made with raw silk, ravelings of white taffeta, and of common sewing silk; of which the first has the finest fibre, the second less fine, and the third, from being twisted and harder, is much coarser. The difference between these three modifications of the same substance was very striking; the raw silk detaining the heat for 1284 seconds, the taffeta ravelings 1169 seconds, and the silk thread only 917 seconds.

The application of these facts to the retention of the natural heat of the body by different kinds of clothing, is sufficiently obvious; and it is evident 
that, as far as mere conducting power of animal and vegetable substances is concerned, the worst conductors are those in which the fibres are finest and most condensed. There are, however, other circumstances which interfere in these applications, and which will be more intelligible when the subject of radiation of heat is under consideration.

\$ 8.-Of the conducting Powers of Liquids.

From all experiments that have been made upon this subject, it appears that liquids are very imperfect conductors of heat; so imperfect, indeed, that they have frequently been regarded as absolute nonconductors; and the same remark applies to gaseous or aëriform bodies. It has already been shown (page 47), that in the usual mode of heating liquids, the heat is applied to the bottom of the vessel containing them, and is diffused over the whole mass in consequence of successive changes of density in the heated portion; the warm currents which are thus established, ascend, and the cold ones descend, and this motion is kept up till the water boils. If, however, we apply heat to the surface of the water, the heat can only be propagated downwards by its conducting power; for the heated portion, being lighter than the colder water beneath, floats upon its surface, and no interchange of strata, analogous to that previously described, can possibly ensue. It may thus be shown that it is almost impossible to transmit heat through water or other fluid by its mere conducting power. The following, among other experiments, may be selected in illustration :-Let $a$ represent a cylin- 
drical vessel of water, with an included air thermometer, the bulb of which very nearly reaches the surface: $b$ is a small copper basin floating upon the surface, the bottom of which is only separated from the bulb of the air thermometer by a very thin stratum of water: if a little glowing charcoal be now put into the basin, the surface of the water may be heated to its boiling point, and yet scarcely any elevation of temperature will be indicated by the neighbouring thermometer; and it will be impossible to transmit the heat from the

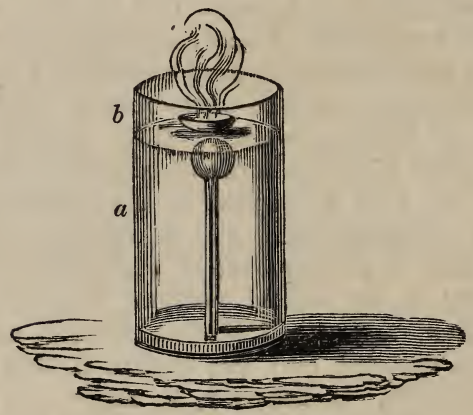

surface downwards. In this way of making the experiment, it is obvious that some heat may be conveyed by the sides of the vessel; but as glass is a very bad conductor, this source of error is trifling, and only produces an effect when the heat is continued for a very long time on the surface.

The following is a mode of illustrating the bad conducting power of another liquid-namely, oil. Let $a$ represent a thin glass tube about two feet in 
length, closed at one end, and open at the other: pour into it a couple of inches of water, and freeze it by immersion in a mixture of salt and ice ; then upon the lump of ice at the bottom, $b$, pour eight or ten inches of oil, $c$, and, upon the oil, two or three inches of spirit of wine, $d$. If

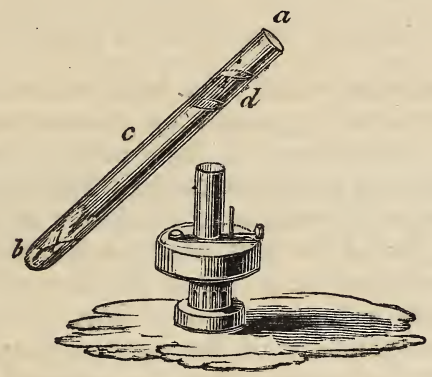

the portion of the tube containing the spirit be now carefully held over the chimney of a lamp, the spirit may be boiled, and even entirely evaporated, whilst the oil will not be sensibly heated, nor the ice thawed.

These, and a number of similar facts and experiments, shew that water, if not a non-conductor, is, at all events, a very bad and imperfect conductor of heat; and that fluids acquire and lose heat principally by the currents which have been above described (page 47), and which always take place in them when partially heated or cooled; the warmer parts ascending, and the colder descending. Whatever impedes these motions will proportionably interfere with the heat- 
ing and cooling of the fluid. Hence viscid liquids, or those containing substances that mechanically thicken them, are more tardy in their reception and emission of heat than pure water: of this, thick soups afford a familiar example; they are a long time cooling.

\$9.-Of the Conducting Powers of Air in regard to Heat.

The experiments which have been made to determine the conducting powers of gaseous bodies, are not very satisfactory in their results; and it has as yet scarcely been ascertained whether air is, or is not, properly speaking, a conductor of heat.

When a heated body is suspended in the atmosphere, and freely exposed on all sides to air, it gradually parts with its excess of heat, so as to . attain the temperature of the surrounding substances; but this cooling effect is produced by a variety of causes, independent of any real conducting power of the air. We have already alluded to the extreme facility with which currents are formed in air when heated substances are exposed to it: the warm air ascends, and a continual current is produced, which glides over the surface of the heated matter; thus, much heat is conveyed away by the establishment of currents, in consequence of change of density in the air. But heated bodies exposed to air also part with excess of temperature by radiation, a process continually going on from their surfaces, and which will presently require our minute attention. Upon the whole, it appears that air is a very imperfect conductor of heat. 
$\$ 10 .-$ Remarks on the relative Conducting Powers of Bodies.

It would appear, from what has been stated, that those bodies which are the best conductors of heat, have the weakest attraction for it, and that bad conductors have the strongest attraction: the former, therefore, part with their heat more readily than the latter. Metals, which are excellent conductors, are easily heated, and as easily give out their heat. It is painful to touch metal heated to about $120^{\circ}$. Water scalds at $150^{\circ}$; but air may be heated to $250^{\circ}$, or even $300^{\circ}$, without being painful to our organs of sensation. Mr. Chantrey has a large stove, or oven, for drying the moulds employed in his bronze castings; a thermometer suspended in it generally stands at about $300^{\circ}$ : the workmen enter and remain in it for some minutes without much inconvenience; and a person wholly unused to such high heats may walk into and out of it with impunity, though, to such, any attempt to remain generally occasions difficult breathing, and a painful sensation about the eyes, but the skin remains parched and dry. In subjecting ourselves to atmospheres thus highly heated, it is necessary carefully to avoid the contact of any good conductor of heat: if we touch a piece of metal, it would inevitably burn us ; and a person once inadvertently entered with his spectacles on, which, being mounted in silver, presently burned the points of contact with his face.

The relative conducting powers of metal and wood are familiarly illustrated in the application 
of wooden handles to metallic vessels intended to contain hot liquids; or, sometimes, where the handle is required to be of metal, a thin piece of wood or ivory is interposed between it and the body of the vessel, by which all rapid communication of heat is effectually cut off.

We avail ourselves of the bad conducting powers of brick, and stone, in the construction of furnaces intended to retain their interior heat; but where the object is to communicate heat to the surrounding air, and not to retain it in the body of the furnace, they are made of cast or wrought iron, and the lining of brickwork is dispensed with, so that the heat may readily find its way outward.

Charcoal is among the very worst conductors of heat; and there is a well known experiment which illustrates this, consisting in placing a teakettle of boiling water, just taken from the fire, upon the palm of the hand: it is, of course, essential to its success that the bottom of the kettle should be well covered with soot, which is a porous form of charcoal, and effectually prevents the transmission of heat from the water within to the hand.

The comparative conducting power of wood and metal is also well illustrated by the following experiment:- - take a smooth round bar of brass, or other metal, about an inch in diameter, and ten or twelve in length, and stretch a strip of writing paper tightly over a part of it, to which apply the flame of a candle or spirit-lamp, and it will be found impossible to burn or even singe the paper; for the heat passes through it to the metal, and is conducted away so rapidly, that its accumulation, or 
the temperature required to burn the paper, cannot be attained. If we now substitute a wooden bar of the same dimensions as the metallic one, stretch the paper over it, and hold it in the flame, the paper will presently singe and char, and the wood beneath will be inflamed, in consequence of its bad conducting power, which prevents the diffusion of the heat, and concentrates its effects upon the spot to which the flame is applied.

A stratum of confined air is an extremely bad conductor of heat: hence the advantage, in some instances, of double walls to furnaces, air being confined between them; and to icehouses, to exclude external heat; and of double windows to our apartments, to prevent the transmission of heat, and ingress of cold.

We have shewn, that in air, the power of communicating heat is very feeble; its actual power of abstracting heat is also proportionably small: in the high northern latitudes, provided the air is tranquil, and there is no wind, a degree of cold may be sustained without injury, in which mercury readily freezes; but if, in this state of the atmosphere, metallic substances be touched, of the same low temperature, a sensation like that of burning is experienced, and the part becomes blistered.

We have above adverted to the effects of a high temperature upon the human body. The opposite effects of a very low temperature cannot be commanded artificially, and, therefore, opportunities seldom occur of witnessing them so as minutely to observe their consequences. Captain Parry and his associates, in their ' Voyage for the Discovery of a North-west Passage,' frequently experienced a 
degree of atmospheric cold below the freezing of mercury. Under the date of Tuesday, the 15th of February, 1820, he says, "From four P.M., on the 14th, till half-past seven on the following morning, being an interval of fifteen hours and a half, during which time the weather was clear, and nearly calm, a thermometer, fixed on a pole between the ships and the shore, never rose above $-54^{\circ}$, and was once during that interval, namely, at six in the morning, as low as $-55^{\circ}$. This low temperature might perhaps have continued much longer, but for a light breeze which sprung: up from the northward, immediately on which the thermometer rose to $-49^{\circ}$, and continued still to rise during the day, till, at midnight, it had reached $-34^{\circ}$. During the lowest temperature above-mentioned, which was the most intense degree of cold marked by the spirit thermometer during our stay in Winter Harbour, not the slightest inconvenience was suffered from exposure to the open air by a person well clothed, as long as the weather was perfectly calm; but in walking against a very light air or wind, a smarting sensation was experienced all over the face, accompanied by a pain in the middle of the forehead, which soon became rather severe. We amused ourselves in freezing some mercury during the continuance of the cold weather, and by beating it out on an anvil previously reduced to the temperature of the atmosphere ; it did not appear to be very malleable when in this state, usually breaking after two or three blows of the hammer.'

* The reader will observe that these temperatures are all below 0 of Fahrenheit's scale., 
\$11.-Of the Effects of Heat in changing the form of Bodies.

Having described the principal phenomena attendant upon the expansion of bodies by heat, and having noticed the manner in which it is propagated through liquids and gases by changes in their densities, and through solids by their conducting powers, we may now proceed to a very important part of the history of this power of matter-namely, to its effects upon the forms or states of bodies.

Common experience tells us, that when solids are exposed to a certain degree of heat, they change their state, and are converted into liquids; and liquids, under the same circumstances, are changed into vapours or elasticfuids : these again, by the loss of heat, revert to the liquid state ; and liquids, by due degrees of cold, are solidified. Ice, water, and steam, are familiar instances of these three states of matter, and the phenomena attendant on their production may be discussed under the heads Liquefaction and Vaporisation.

One very important fact respecting the conversion of ice into water has already been mentioned, in describing the method of graduating thermometers (page 55), namely, that, during the progress of thawing, the ice and the water always maintain an uniform temperature, which is equal to $32^{\circ}$ of Fahrenheit's scale. Suppose, for instance, that we bring some pounded ice from a very cold place (say of the temperature of $0^{\circ}$ ) into a room of the temperature of $60^{\circ}$, the ice, of course, soon be- 
gins to liquefy, and a thermometer plunged into it rises from $0^{\circ}$ to $32^{\circ}$; but there it remains stationary until the whole of the ice has melted: the water thus formed, then gradually rises to $60^{\circ}$, which is the temperature of the surrounding air. Now it is obvious, that during the whole process of thawing under the circumstances above described, heat must be entering the ice, but its effect is, not to elevate its temperature, but to melt it: the heat apparently combines with the ice, to constitute water, but it does not affect the thermometer.

To heat thus united with bodies, Dr. Black gave the name of latent or combined heat; and he instituted a number of elegant experiments upon the subject of liquefaction and congelation, one or two of which may be here detailed: they are at once simple and satisfactory.

Two similar glass globes were filled, the one with five ounces of water, which was afterwards frozen, and the other with water cooled down to between $32^{\circ}$ and $33^{\circ}$, but not suffered to congeal. That which contained the ice was allowed to remain in a warmer air, till the ice began to melt, at which time its temperature was as nearly as possible $32^{\circ}$ or $33^{\circ}$. The two vessels were then suspended in the middle of a large room without a fire, or any notable draught of air, and of the average temperature of $47^{\circ}$. The vessels, therefore, were in every respect under similar circumstances of temperature within and without, and with similar contents, except that the one contained ice, and the other water. The progress of heating was then noted. 
In that which held the water, the thermometer rose in half an hour to $40^{\circ}$; but, in the other, it took no less than ten hours and a half before all the ice was melted and the contents had reached to $40^{\circ}$. Consequently, the rate of heating was twenty-one times slower in the ice vessel than in that which held the cold water; but the actual heat received by the former was obviously nearly uniform the whole time; and, therefore, it required about twenty-one times as much heat to bring ice to the state of water, and to raise it to $40^{\circ}$, as to raise ice-cold water to the same point. Or, to express it by another form,-if the water had continued to receive as great an influx of heat as the ice during the whole twenty-one half hours, its temperature would, of course, have been raised twenty-one times as much as during the single half hour; and, as this was $7^{\circ}$, the whole would have been twenty-one times $7^{\circ}$, or $147^{\circ}$. The difference between the gain of temperature in the ice and the water, with equal accessions of heat, is therefore about $140^{\circ}$; and thus $140^{\circ}$ is the expression of that quantity of thermometric heat, or temperature, which disappears, or is rendered latent by the separate operation of the liquefaction of ice; the actual quantities of matter in the comparison being equal *.

The conclusions drawn from the preceding experiment on the slow thawing of ice, were verified and confirmed by melting it rapidly by mixture with hot. water. Thus, if we take any quantity of finely pounded ice, or of snow, at 
the temperature of $32^{\circ}$, and mix it with an equal weight of hot water, at the temperature of $172^{\circ}$, the whole of the snow immediately melts, and the temperature of the resulting water is only $32^{\circ}$. Here, therefore, the water suddenly loses $140^{\circ}$ of heat, while the temperature of the snow remains as at the outset of the experiment, and is not at all increased. So that $140^{\circ}$ of heat have disappeared; they have combined with the snow; and their effect has been, not to raise its thermometric temperature, but to convert it into water. Ice, therefore, when it is converted into water, absorbs and combines with a certain proportion of sensible heat, and renders it latent. The exact number of thermometric degrees of heat which disappear during the liquefaction of ice have been somewhat differently estimated by different experimentalists. The mean of the whole is about $140^{\circ}$, which also is Dr. Black's inference.

From the preceding experiments we deduce this important general law-that in all cases of liquefaction, a quantity of heat not indicated by, or sensible to, the thermometer, is absorbed or disappears; and that this heat is withdrawn from the surrounding bodies, leaving them comparatively cold.

The cases which we have cited, are those of liquefaction at comparatively low temperatures, and relate to ice and water; but the same laws are observed in all other cases, and where the temperatures are comparatively high: to these, the term fusion is generally applied: spermaceti, for instance, fuses at $112^{\circ}$, and retains that temperature during its fusion so long as any portion 
remains unmelted: when the whole is liquid, and not till then, its temperature may be elevated above the fusing point. Again; a ladleful of lead cannot be heated red hot till all the metal is melted; and a quantity of red-hot melted lead is instantly cooled down to its point of fusion (which is about $606^{\circ}$ ) by the addition of a piece of solid lead.

That the heat which becomes insensible or latent during the liquefaction of ice and other solids, remains in the liquid, and is not altogether lost or destroyed, is shewn by the circumstance of its being again emitted or given out during their congelation. If a vessel of warm water be exposed in a very cold atmosphere, the temperature rapidly sinks to the freezing point, and then it begins slowly to congeal: if kept in the same situation, heat is of course abstracted as before; but the thermometric heat remains stationary at about $32^{\circ}$, till the whole is frozen, after which, the temperature begins again to sink as before.

Perhaps the result of this experiment is more explicitly stated as follows* :-If, when the thermometer is at $22^{\circ}$, we expose a vessel full of water at $52^{\circ}$ to the open air, and beside it another vessel full of brine, at the same temperature, with thermometers in each, we shall find that both of them gradually lose heat, and are cooled down to $32^{\circ}$. After this, the brine (which does not freeze till cooled down to $4^{\circ}$ ) continues to cool without interruption, and gradually reaches $22^{\circ}$, the temperature of the air; but the pure water remains 
stationary at $32^{\circ}$ : it freezes, indeed, but very slowly; and during the whole process its temperature is $32^{\circ}$. Now why should one liquid refuse, all of a sudden, to give out heat, and not the other? Is it not much more probable that the water, as it freezes, gradually gives out the heat which it had absorbed during its liquefaction, and that this evolution maintains the temperature of the water at $32^{\circ}$, notwithstanding what it parts with to the air during the whole process? It is easily shewn that water, while congealing is constantly imparting heat to the surrounding air ; for a delicate thermometer suspended above it is constantly affected by an ascending stream of air less cold than the air around.

The following experiment also shews that this evolution of heat actually takes place during congelation. If, when the air is at $22^{\circ}$, we expose to it a covered glass of water, with a thermometer in it, the water, if kept quite still, may be gradually cooled down to $22^{\circ}$ without freezing. It is, therefore, ten degrees below the freezing point. If the water be now shaken, part of it instantly freezes, and the temperature of the whole rises to $32^{\circ}$; so that the water acquires $10^{\circ}$ of heat in an instant. Now, whence comes this heat? Is it not evident that it is derived from that part of the water which suddenly froze; and, consequently, that water, in the act of freezing, gives out heat?

There are many instances of the sudden or rapid liquefaction of bodies, in which the absorption of heat, or production of cold, are more remarkable and striking than in the cases above 
detailed. When finely powdered common salt, for instance, is mixed with pounded ice or snow, a sudden liquefaction of the solids ensues, and a proportionate production of cold; they immediately run down into brine, which does not freeze till reduced to near $0^{\circ}$; and a thermometer plunged into the mixture sinks nearly to that point. We sometimes avail ourselves of this process, to remove hard trodden snow from the pavement in winter: it is soon liquefied by sprinkling a little salt upon it, and the temperature of the air is seldom low enough to freeze the brine which is produced. Confectioners employ the mixture of ice and salt to freeze cream in the hot weather of summer; and the same system is conveniently resorted to whenever considerable cold is required.

In these cases, the disappearance of the heat is caused by the sudden formation of liquid brine from its solid constituents; and the bodies being already cooled to $32^{\circ}$, the thermometer will sink about $30^{\circ}$. It was once supposed that this was the lowest temperature that could be artificially commanded ; and hence, probably, our thermometric scale sets out with it: but we now know that, by careful management, the mixture of certain materials will afford a much more intense cold. Among the most effective of these is the mixture of snow and the salt called chloride of calcium, or muriate of lime: the snow should be taken in its light flocculent state, as it falls in a very cold winter's day ; and the chloride, in fine powder, but not deprived of its water of crystallization, should be mixed with it in the proportion of 
about three parts to two: the mixture should be made in earthen vessels, previously cooled, and in the lowest atmospheric temperature we can command; the thermometer will then sink from $32^{\circ}$, to between $40^{\circ}$ and $50^{\circ}$ below $0^{\circ}$, a temperature at which mercury freezes. By the successive application of freezing mixtures in a proper apparatus, Mr. Walker succeeded in sinking the spirit thermometer to $-91^{\circ}$; a temperature at which all known liquids, with the single exception of pure alcohol, are converted into the solid form. The following table shews some of the most useful of these freezing mixtures, and the temperatures which result from their careful application.

\begin{tabular}{|c|c|}
\hline Mixtures. & Thermometer sinks. \\
\hline \begin{tabular}{lccc} 
Muriate of ammonia & \multicolumn{1}{c}{ Parts } \\
Nitre & $\cdot$ &. & 5 \\
Water & $\cdot$ & $\cdot$ & $\cdot 16$
\end{tabular} & From $50^{\circ}$ to $10^{\circ}$. \\
\hline $\begin{array}{l}\text { Nitrate of ammonia }: 1 \\
\text { Water } \quad \cdot \quad \cdot 1\end{array}$ & From $50^{\circ}$ to $4^{\circ}$. \\
\hline $\begin{array}{l}\text { Sulphate of soda } \\
\text { Diluted sulphuric acid }\end{array} \quad \begin{array}{l}5 \\
4\end{array}$ & From $50^{\circ}$ to $3^{\circ}$. \\
\hline 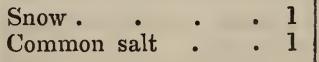 & From $32^{\circ}$ to $0^{\circ}$. \\
\hline 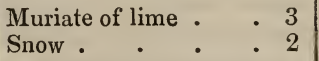 & From $32^{\circ}$ to $-50^{\circ}$ \\
\hline $\begin{array}{ll}\text { Snow } & 2 \\
\text { Diluted sulphuric acid } & 1 \\
\text { Diluted nitric acid } & 1\end{array}$ & From $-10^{\circ}$ to $-56^{\circ}$, \\
\hline
\end{tabular}




\begin{tabular}{|c|c|c|}
\hline \multicolumn{2}{|l|}{ Mixtures. } & Thermometer sinks. \\
\hline $\begin{array}{l}\text { Snow or pounded ice } \\
\text { Common salt } \\
\text { Nitrate of ammonia }\end{array}$ & $\begin{array}{l}\text { Parts } \\
.12 \\
. \quad 5 \\
. \quad 5\end{array}$ & From $18^{\circ}$ to $-25^{\circ}$. \\
\hline $\begin{array}{l}\text { Muriate of lime . } \\
\text { Snow . }\end{array}$ & $\begin{array}{l}3 \\
\cdot \quad 1\end{array}$ & From $-40^{\circ}$ to $-73^{\circ}$. \\
\hline $\begin{array}{l}\text { Diluted sulphuric acid } \\
\text { Snow . }\end{array}$ & $\begin{array}{r}10 \\
. \quad 8\end{array}$ & From $-68^{\circ}$ to $-91^{\circ}$. \\
\hline
\end{tabular}

Whenever ice can be procured, its mixture with common salt furnishes the cheapest and most effectual of all the freezing mixtures applicable to the common purposes of life; but when it cannot be obtained, we often conveniently avail ourselves of the two first saline combinations in the preceding table: either the mixture of muriate of ammonia and nitre, or of nitrate of ammonia and water, may be economically used, and the salts may again be obtained in a fit state for repeated use, by evaporation. In employing them to cool a bottle of champagne, for instance, a vessel should be selected a little larger, and nearly as tall as the bottle; it should then be filled with the coldest pump-water that can be procured, and the bottle placed in it : about four ounces of the salt in fine powder should be sprinkled upon the shoulder of the bottle, so as, gradually dissolving, to fall or run down its sides; as the salt dissolves, the bottle should be gently turned in the mixture, and kept in it till an immersed thermometer tells us that the temperature 
is rising, which will be in about twenty minutes or half an hour.

As liquefaction is to the surrounding bodies always a cooling process, and as sudden liquefaction produces a sudden degree of cold, so solidification or congelation is to surrounding substances a heating process; and when it takes place suddenly, a proportionably sudden elevation of temperature is the consequence. In the experiment described in page 16, a solution of Glauber's salt is made suddenly to crystallize; and if the hand be applied to the flask, it will be found to become sensibly warm. When water is poured upon quick-lime, a sudden solidification of a large portion of water is effected, in consequence of its attraction for the lime, which crumbles down into a dry white powder, and a great evolution of heat ensues, as we see by the steam which makes its escape in the ordinary operation of slaking lime.

From the view, then, which we have taken of the constitution of liquids, it is obvious that they may be considered as combinations of solids with heat; that, consequently, when they revert to the solid state, they give out their heat of fluidity, which, previously latent, or insensible to the thermometer, now becomes sensible, in consequence of the change of state: on the other hand, when solids are converted into liquids, they absorb or combine with that portion of heat which is requisite to their respective fluidities.

We nay now proceed to examine the effects of heat upon liquids.

When liquids are heated, they are converted with more or less facility into invisible elastic 
fluids, or vapours. When water is heated in open vessels, its temperature gradually rises, and vapour is seen to form on its surface, which increases till the thermometer rises to about $212^{\circ}$, which is called the boiling point of water; it is then violently agitated, and gradually disappears in the form of steam: but, although the source of heat is continued, the temperature of the water remains stationary, and both it, and the steam, continue at $212^{\circ}$. There are, therefore, in this simple operation, two leading circumstances to be attended to, which are very important in their application and consequences: these are, the degree of expansion which the water suffers by conversion into vapour; and the cause of that uniformity of temperature to which we have adverted, and which has been above noticed as furnishing one of the fixed points for thermometric graduation. (See page 56.)

In regard to the bulk of steam formed by a given bulk of water, it appears from the experiments of Gay Lussac, that a pint of water is convertible into 1689 pints of steam, at the temperature of $212^{\circ}$ (the barometer standing at 30 inches). Mr. Watt conceived the bulk of steam to be about 1800 times that of the water; and others have far exceeded even Watt's estimate: we may, however, adopt that of Gay Lussac as the most accurate.

Steam is an invisible elastic fluid like common air, and possessed of similar mechanical properties. We are, it is true, in the habit of associating a smoky appearance with steam, because we generally observe it when it is beginning to be 
condensed; as when it escapes, for instance, from the spout of a teakettle; but, when perfectly formed, it is quite invisible, as may be shown by boiling water in a flask, when perfect transparency will exist in the upper part of the vessel which is filled with the hot vapour; and it only becomes visible when it escapes into the air, and suffers incipient condensation.

The same general fact, in regard to the visibility of vapour, is shown by the production of fogs, which are occasioned by the mixture of warm with cold air; the general depression of temperature thus produced, rendering the aqueous vapour visible, in consequence of its partial condensation. This partial condensation does not amount to the actual precipitation of water, or rain; but consists of a multitude of thin vesicles; and hence the appearance is often called vesicular vapour: they constitute clouds, mist, dew, and fogs. Clouds, therefore, are formed, whenever two masses of air, of different temperatures, and each saturated with moisture, meet and mix together; but what it is that subsequently causes the condensation of the vesicular vapour, and its precipitation in the form of rain, is not exactly known.

Steam, as we have said, is produced by heating water to $212^{\circ}$ under common barometrical pressure, the thermometer remaining fixed at that point, which is, therefore, called the boiling point of water. But this point, or temperature, varies with, and is immediately dependent upon, the pressure to which the surface of the water is exposed; it is also, to a small extent, affected by the nature of the vessel containing the water. 
In a metallic vessel, when the barometer stands at 30 inches, pure water boils at $212^{\circ}$; but when the barometer falls to 29 inches, the boiling point of water is lowered very nearly to $210^{\circ}$. Of course, therefore, we find a very considerable diminution in the boiling point of water upon the summits of hills and mountains, and other elevated situations; and a very delicate thermometer indicates a lowering of the boiling point even at the top of high buildings. Saussure found that, at the top of Mont Blanc, water boiled at $187^{\circ}$. Now, it has been shown that, for every half inch of variation in the barometer, the boiling point of water varies eight-tenths of a degree; consequently, every tenth of an inch that the barometer rises or falls, either elevates or depresses the boiling point by 0.176 of a degree.

These circumstances respecting the boiling point of water are here mentioned, to show that even the ordinary changes in the height of the barometer will materially affect it. In this country the mean height of the barometer is a little short of 30 inches: it very rarely rises higher than 31 inches, or falls below 28.5 ; so that from this cause alone, there will be a variation in the boiling point of water of between four and five degrees.

But if, as has already been remarked, the pressure be more considerably diminished, by artificial methods, the temperature at which liquids boil will be proportionately lowered; and hence we often use the terms boiling and ebullition to signify the rapid conversion of water into vapour, independent of the temperature at which it takes 
place: using the term in this sense, we shall presently have occasion to point out a case of boiling, at the freezing temperature.

The air-pump is a very useful instrument for showing the influence of diminished pressure upon boiling points. If, for instance, we place a glass of water of the temperature of $90^{\circ}$ or $100^{\circ}$ under the receiver of the air-pump, and remove the air's pressure upon it, we shall observe that the water soon boils violently at that temperature; and would continue so to do, but that the space in the receiver becomes filled with an atmosphere of vapour, which, exerting its pressure upon the water, again prevents ebullition.

If we use liquids which, under ordinary circumstances, boil at temperatures below that of water, we shall find them boiling in vacuo at proportionately low degrees; this is the case with ether and with alcohol, which, under the above circumstances, readily boil at all common temperatures.

It may not be improper here to observe, that we are in the habit of applying the term vapour to all those elastic fluids which are easily reconvertible into liquids: steam is easily converted into water by lowering its temperature a few degrees; and those liquids which have low boiling points are especially convenient for illustrating these changes ; such, for instance, as ether or alcohol ; and as ether is only sparingly soluble in, and much lighter than water, it may conveniently be used in the following simple, but illustrative experiment.

Nearly fill a glass tube about three feet long, and half an inch diameter, with water; then pour 
a little ether upon the surface of the water; and closing the open end of the tube carefully with the palm of the hand, invert it in a basin of water, as shown in the annexed cut: the ether will

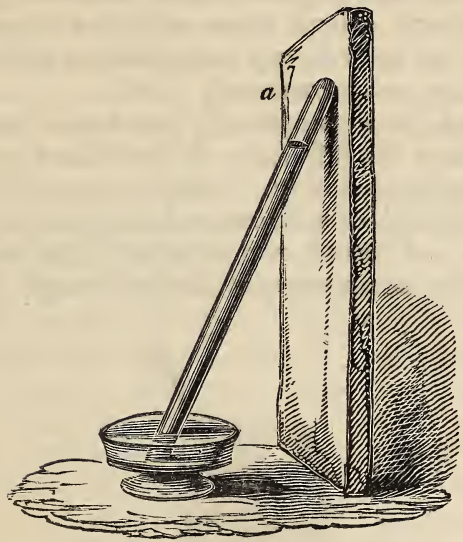

rise through the water, and occupy the upper end of the tube, $a$; retaining, at common temperatures, its liquid state ; but if we now pour a little hot water upon the exterior of the tube, it presently causes the ether within, to boil, and its vapour may thus be made to drive nearly the whole of the water out of the tube into the basin below : the ether retains this elastic or vaporous state, so long as its temperature is sufficiently high; that is, above $100^{\circ}$; but if we now cool the tube, by suffering some cold water to dribble over it, we shall observe the etherial vapour return to its liquid state, and float upon 
the water as before. At temperatures above $212^{\circ}$, water would be in the same predicament as ether.

With regard to the bodies usually called gases, they are not thus easily condensible: a few of them become liquids when considerably cooled, and several others when subjected to great pressure; but at ordinary temperatures and pressures the gases are permanently elastic.

The following apparently paradoxical experiment illustrates the influence of diminished pressure in facilitating ebullition. Insert a stop-cock securely into the neck of a Florence flask containing a little water, and heat it over a lamp till the water boils, and the steam escapes freely through the open

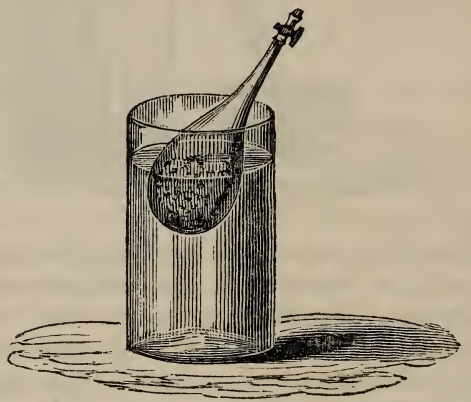

stop-cock; then suddenly remove the lamp and close the cock; the water soon ceases to boil; but if the flask be now plunged into a vessel of cold water, ebullition instantly recommences, but it again ceases on holding the flask near the fire, or over the lamp. In this experiment the 
air is driven out of the flask, and an atmosphere of steam included within it, which, upon irnmersion in cold water is condensed, and a vacuum formed above the water, which, upon the principle already noticed, boils under the diminished pressure: the vacuum is kept up by the successive condensations of the portions of steam formed, and the boiling goes on as long as the water in the flask remains sufficiently warm; but if it be removed from the cold medium, and especially if it be warmed, a new atmosphere of steam is formed, the pressure of which prevents the recurrence of ebullition.

Boiling in a more or less perfect vacuum, is sometimes resorted to upon a large scale in the arts; it has been very successfully applied to the refining of sugar, the syrup being thus concentrated to the granulating point, without the risk of burning or decomposing it by any excess of heat. The same plan has also been resorted to in the Pharmaceutical Laboratory, in the preparation and distillation of certain medicinal substances, the properties of which are easily impaired by heat.

If we now reverse the state of things which has just been explained, and expose liquids to an increased pressure, we shall proportionately elevate their boiling points. Papin contrived an instrument for this purpose, which was called a digester. It was a cylindrical copper vessel, having a lid very nicely fitted to it and secured by screws. If such a vessel be about half filled with water, with the lid closely secured, and then put upon the fire, steam is soon formed; but, having no escape, it presses upon the water, and prevents the further 
formation of steam till the temperature of the water rises above the boiling point. This heat being conveyed to the steam, it now receives another portion of vapour without being condensed, and thus the quantity and the elasticity of the steam are continually increasing with the temperature. Water has in this way been raised to the temperature of $419^{\circ}$, or even above it; for Muschenbroek asserts that he raised the temperature of the water high enough to melt tin, which requires a temperature of $440^{\circ}$.

At the temperature of $419^{\circ}$, the elasticity of steam is 1050 times greater than that of atmospheric air; so that it exerts upon the inside of the vessel in which it is pent up, a force of at least 14700 pounds on each square inch; a pressure so enormous, that few vessels can resist it, and, consequently, the source of many serious accidents ; which, in the applications of high-pressure steam, are guarded against by safety-valves and other similar contrivances*.

For experiments of this kind upon a small and safe scale, the following is a good form of apparatus :- $a$ is a strong brass globe, composed of two hemispheres screwed together with flanches; a portion of quicksilver is introduced into it, and it is then about half filled with water: $b$ is a barometer-tube passing through a steam-tight collar, and dipping into the quicksilver at the bottom of

* The powerful effects of high-pressure steam are illustrated upon a small scale by the little glass bubbles commonly called candle or fire-crackers; they are hermetically sealed, and contain a drop of water, which occasions them to burst with great violence when sufficiently heated. 


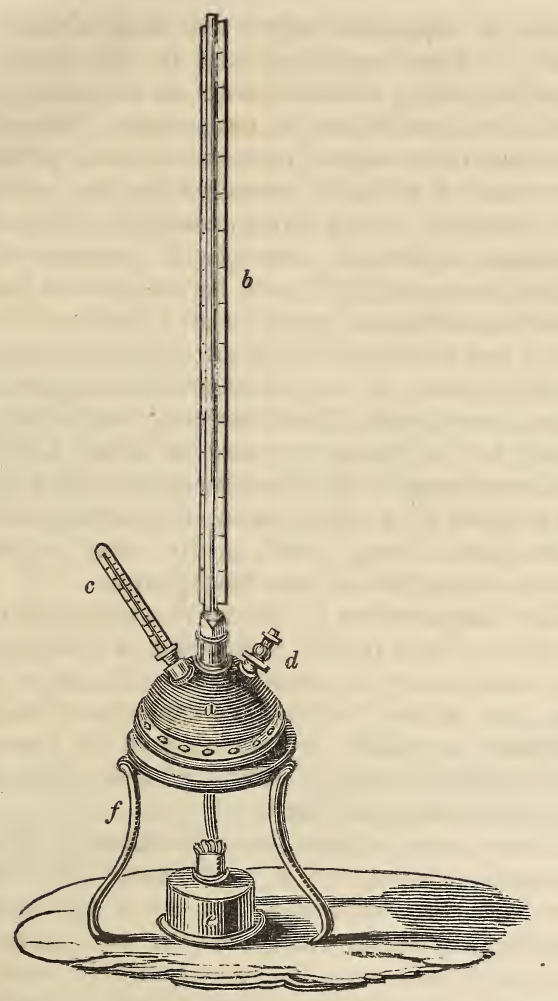

the globe: $c$ is a thermometer graduated to about $400^{\circ}$, and also passing through an air-tight collar: $d$ is a stop-cock, and $e$ a large spirit-lamp. The 
whole is supported upon the brass frame and stand $f$. Upon applying heat to this vessel, the stop-cock being closed as soon as the water boils, it will be found that the temperature both of the water and of its vapour, increases with the pressure, the extent of which is measured by the ascent of the mercury in the barometer-tube. The thermometer, under an atmospheric pressure of 30 inches, being at $212^{\circ}$, will be elevated to $221.6^{\circ}$ under an additional pressure of 5 inches of mercury ; and to $269.6^{\circ}$ under an additional pressure of 30 inches, or one additional atmosphere or thereabouts; each inch of mercury, above 30 , producing by its pressure a rise of about $1.92^{\circ}$ in the thermometer. The barometer-tube also serves the purpose of a safety-valve, the strength of the brass globe being such as to resist a greater pressure than that of one atmosphere.

The temperature of steam is always the same as that of the liquid producing it; hence the high temperature of steam generated under pressure: and as this elevation of temperature may be accurately regulated by the degree of pressure, high-pressure steam is often conveniently applied as a source of steady heat.

We are now to shew, more particularly, that the production of vapour is in all cases attended by loss of sensible heat; and that, as in the case of liquefaction, heat disappears, to constitute the liquid, so, in all cases of evaporation, heat becomes, to a much greater extent, latent in the vapour.

The fact already adverted to, that we cannot, under ordinary circumstances, raise the temperature of water above $212^{\circ}$, although heat continues 
as before to flow into it, shews that the heat must be latent in the steam, the temperature of which does not, however, exceed that of the water which produces it: and again, when water, as in the preceding experiment with the brass globe, is heated under pressure to a temperature above its ordinary boiling point, the moment that that pressure is removed by suddenly opening the cock, a quantity of steam rushes out with great violence, and the remaining water has its temperature immediately lowered to $212^{\circ}$. The quantity of water in this case, which constitutes the lost steam, is very small, yet it carries off the whole excess of heat from that remaining in the globe.

Dr. Black instituted some very ingenious, and, at the same time, simple experiments, to determine the actual loss of heat during the conversion of water into steam*. He heated water in a tin vessel up to its boiling point, and noted the time required for the purpose. The same heat was then continued till the whole of the water was evaporated; and the time taken up by that process was also noted. Now, since, on the one hand, the accession of heat was constant, it was easily computed how high the temperature would have been, supposing the rise to have gone on above $212^{\circ}$, in the same ratio as below it; and, on the other, as the temperature of the steam was not raised, it was inferred that all the accession of heat from $212^{\circ}$ was essential to the very state and constitution of steam at that temperature; this quantity was estimated at about $810^{\circ}$; that is to 
say, that the same quantity of heat which is required totally to evaporate boiling water at $212^{\circ}$, would be sufficient to raise the water $810^{\circ}$ above the boiling point, or to $1022^{\circ}$, if it had remained in the liquid state.

On the other hand, when steam is again comdensed into water, it necessarily gives out the latent heat which was essential to its state of vapour, and which, being then set free, will raise the temperature of adjacent bodies as much more than an equal weight of boiling water would do, as the latent heat of steam exceeds that of the water.

The small boiler represented in the annexed cut may be conveniently employed in these experiments on the latent heat of steam*.

For this purpose the tube $e$ must be screwed on
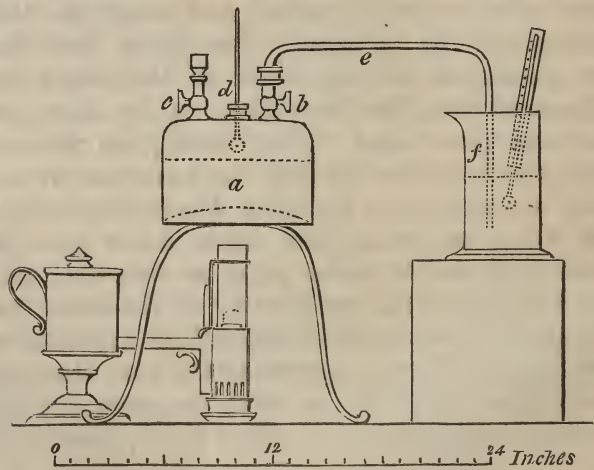

* Henry's Elem. Chim. 
the stop-cock $b$, and immersed into the glass of water $f$. The cock $c$ being closed, the steam arising from the boiling water $a$ will pass into the cold water $f$, the temperature of which will be much augmented by its condensation. Ascertain the increase of temperature and weight, and the result will show how much a given weight of water has had its temperature raised by a certain weight of condensed steam. To another quantity of water, of the same weight and temperature as that in the jar at the outset of the experiment, add a quantity of water at $212^{\circ}$, equal in weight to the condensed steam ; it will be found, on comparing the resulting temperatures, that a given weight of steam has produced, by its condensation, a much greater elevation of temperature than the same quantity of boiling water. If, for instance, 100 gallons of water at $50^{\circ}$ be mixed with 1 gallon at $212^{\circ}$, the temperature of the whole will be raised by about $1 \frac{1}{2}^{\circ}$. But if a gallon of water be condensed from the state of steam in a still tub containing 100 gallons of water, the water will in that case be raised $11^{\circ}$. A gallon of water, therefore, condensed from steam, raises the temperature of 100 gallons of cold water $9 \frac{1}{2}^{\circ}$ more than a gallon of boiling water. If the heat imparted to 100 gallons of water by 8 pounds of steam could be condensed in 1 gallon of water, it would raise it to $950^{\circ}$; and a gallon of water converted into steam of ordinary density, contains as much heat as would bring $5 \frac{1}{2}$ gallons of ice-cold water to the boiling point. The quantity of ice which is melted by steam of mean density is invariably $7 \frac{1}{2}$ times the weight of the steam. 
Having thus explained the circumstances under which vapour is formed at high temperatures, it may be necessary to observe, that precisely the same laws preside over its production at low temperatures ; that the constitution of vapour, therefore, is always the same. When water slowly evaporates under exposure to air, its vapour mixes with the surrounding atmosphere, and the process is usually called spontaneous evaporation; it takes place at all temperatures, and with a rapidity proportionate to the dryness of the air, and the velocity of the current passing over it: thus, in some of our manufactories, where large quantities of liquids are to be evaporated, instead of heating or boiling them in the usual way, a current of hot air is made to pass over the surface of the fluid.

It has just been stated that the vapour slowly formed in cases of spontaneous evaporation, is similarly constituted to that produced by rapid ebullition; that is, there is the same loss or abduction of sensible heat in the one case as in the other. The exhalation of humidity, therefore, which is almost constantly going on from the earth's surface, powerfully modifies natural temperatures, and prevents accumulation of heat; the vapour forms mists and clouds, and when these are condensed into rain, their latent heat is again given out in the upper and cold regions. Hence we observe how important a part vapour performs in natural phenomena, considered as a mere carrier of heat.

By inducing rapid evaporation at common or low temperatures, we have it obviously in our power to produce considerable degrees of cold. 
If a wet cloth is spread out in a keen wind at a temperature a few degrees above freezing, the water, by its rapid evaporation, soon carries off so much heat as to freeze the remainder, making the cloth hard and stiff by the formation of ice within its pores.

There is no liquid better calculated for making experiments on the cold produced by spontaneous evaporation, than common sulphuric ether; it is extremely vaporisable at ordinary temperatures, and if we pour a little of it into the palm of the hand, and blow gently upon its surface, we observe that, as it disappears in vapour, a very sensible degree of cold is produced.

If we fold a strip of cambric round the bulb of a small thermometer, and suffer ether to dribble over it from a dropping bottle, at the same time accelerating the evaporation by blowing gently upon it with the mouth or a pair of bellows, we shall observe the mercury fall to several degrees below the freezing point; and if we substitute for the thermometer a thin glass tube containing a small quantity of cold water, a little dexterous management will enable us by the same means to freeze it.

This congelation of water by the evaporation of ether furnishes, by the help of the air-pump, a very striking experiment. Provide a small thin glass flask, which fits tolerably close into a bellshaped wine-glass, as represented in the next page. Pour a little good ether into the flask, and some cold water into the wine-glass, so that each may stand at about the level $a$, and place the whole apparatus, thus arranged, under the receiver of an air-pump. 
During exhaustion, the ether will boil, that is, it will very rapidly pass off in the state of vapour.

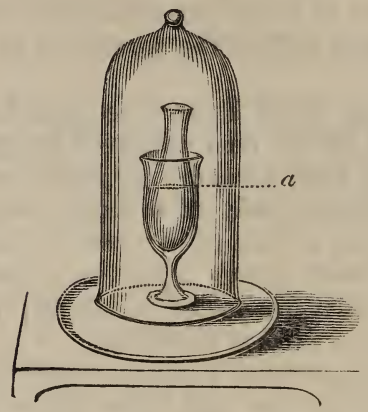

Now, the vapour of ether, like all others, requires heat for its formation, and this it takes from the surrounding bodies, and, among the rest, from the adjacent water, which soon freezes in consequence of the loss of that portion of heat which was essential to its fluidity, but which has been carried away and pumped out in the etherial vapour. It sometimes happens that the success of this experiment is prevented by the ether boiling over into the water, and so preventing its congelation; but this may be guarded against by putting a little bit of wood or paper into the ether, which causes it to boil regularly, and prevents sudden jets of vapour.

There is an experiment yet more illustrative of the absorption and disappearance of sensible heat during evaporation, which consists in freezing water by causing it rapidly to evaporate; it is 
performed as follows : upon the plate of a good airpump place a flat shallow glass dish, A, about half filled with sulphuric acid, and a little above it a tin or copper basin $\mathrm{B}$, three parts filled with water, with a small thermometer $\mathrm{c}$ immersed in it.

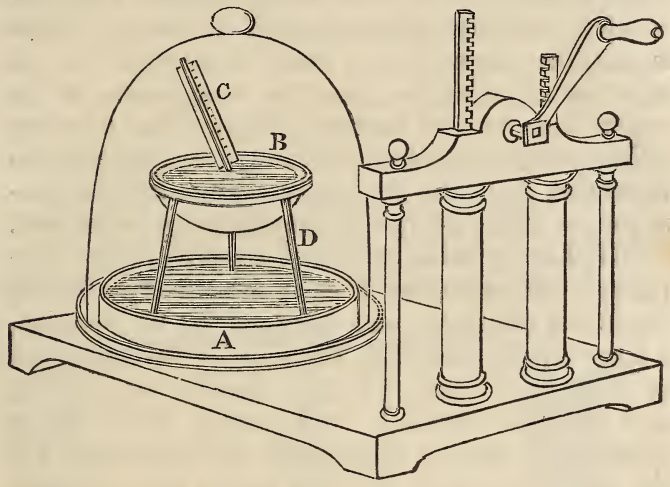

This basin, which should be of the shape shewn in the cut, may be conveniently supported upon three glass legs D, standing in the acid. Over the whole place an air-pump receiver, not larger than requisite, and proceed to withdraw the air. We first observe the escape of air from the water; the thermometer gradually sinks; the water then, in consequence of the rapidity of its evaporation, appears to boil ; and if the whole apparatus is in good order, it freezes in the course of five or ten minutes. The use of the surface of sulphuric acid here, is to absorb the aqueous vapour, which it does very energetically, and consequently occasions a 
constant fresh call upon the water for its formation. Now we have shewn that rapour cannot be produced without the absorption of heat; and, in the case before us, the heat requisite to convert one part of the water into vapour is taken from the other fluid portion, which, thus losing the heat that constituted its fluidity, becomes solid, or freezes. There is another phenomenon often observable in this experiment, which is, that the temperature of the water falls several degrees below the freezing point, before congelation takes place; but the moment that the water freezes, it rises to $32^{\circ}$, in consequence of the escape of the residuary latent heat. Mr. Leslie, who contrived the preceding experiment, has proposed it as a means of procuring ice in hot climates, and suggests for the purpose, a large simple air-pump, capable of exhausting six or eight receivers at a time. He has also shewn, that parched oatmeal, and certain dried earthy substances, may be substituted, as absorbents, for the sulphuric acid; but they are far less effectual. The fact is, that the experiment is very interesting and curious as a philosophical illustration of the effects of vaporization, but does not at present appear susceptible of economical applications.

Dr. Wollaston's cryophorus, or ice-bearer, illustrates the same general facts, in a more simple manner than the preceding; it depends upon the same common principle, but the vacuum and the evaporation are brought about in a different way. A tube of the shape annexed, has a bulb at each

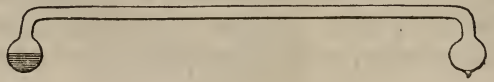


extremity, one of which is half filled with water; the interior of the tube is perfectly exhausted of air, the consequence of which is, that the water in the bulb is greatly disposed to evaporate; but this evaporation can only proceed to a certain extent in consequence of the pressure of vapour within the tube soon preventing its further progress. To get rid of this, to keep up the vacuum, and to occasion a constant demand upon the water for the fresh formation of vapour, the empty ball is plunged into a freezing mixture, which continually condenses the vapour within, and so accelerates the evaporation of the water in the other bulb, as to cause it ultimately to freeze. The success of this experiment, when it is at-

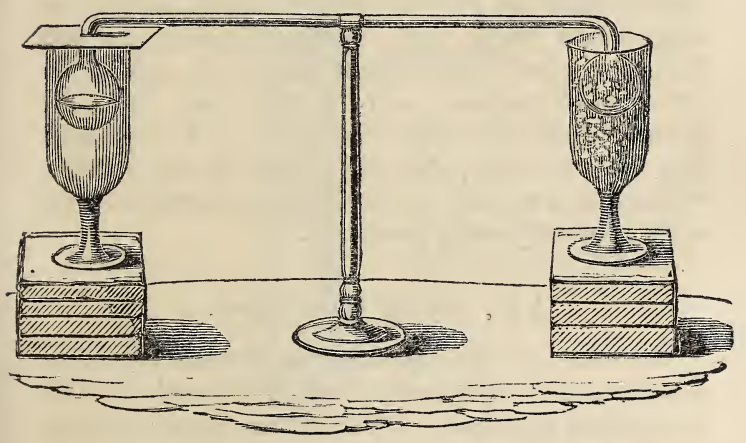

tempted in a warm room, where there are several persons, is often frustrated by the rapid deposition of moisture, arising from the condensation of aërial vapour upon the water-bulb; an effect which may 
be prevented by protecting it from the free access of fresh portions of air, by immersion in a covered glass, as shown in the preceding sketch, which also represents the empty bulb surrounded by a freezing mixture of pounded ice and salt.

The intermediate tube between the bulbs of the cryophorus may be of any length; about two feet is most convenient, for when longer it is very apt to be broken.

There are many cases in which we resort to accelerated evaporation at common temperatures as a source of cold, without, however, carrying it so far as to produce ice. The sprinkling of floors and pavements with water tends to cool them and the air, by its evaporation at summer temperatures: wine-coolers are porous earthen vessels, which are dipped into water, and, during exposure to air, become cooled by its escape from their surfaces in the form of vapour; and in some hot climates, porous bottles are filled with water, and suspended in a current of air ; the water oozes to the surface, whence it is rapidly removed by evaporation, and cold is consequently produced.

\section{§12. - Of the Steam_Engine.}

So many of the phenomena attendant on evaporation and liquefaction are illustrated by the action and principles of the steam-engine, that it may be worth while to refer to the construction and operation of that instrument, as elucidating several points of discussion already adverted to. We are the more induced to this, as the subject is in itself extremely simple and intelligible, and yet 
not so generally comprehended as it should be, considering how much has been effected of late years by these admirable machines.

The action of the steam-engine depends principally upon the two leading properties of steam which have been dwelt upon-namely, its expansive force, and its easy condensation. To take the most simple view of these as moving powers,

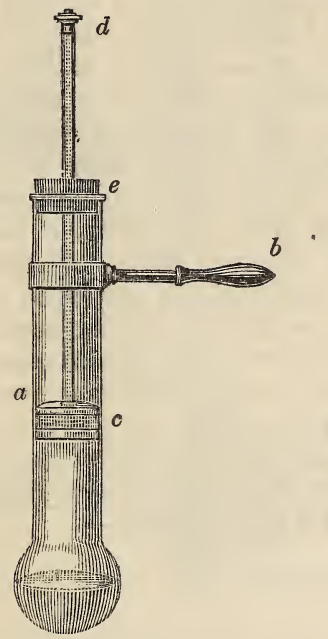

let $a$ represent a glass tube with a bulb at its lower end. It is held in a brass ring, to which a wooden handle $b$ is attached, and contains a piston $c$, which, as well as its rod, is perforated, and may be opened or closed by the screw at top $d$ : it is 
kept central by passing through a slice of cork at $e$. When used, a little water is poured into the bulb, and carefully heated over a spirit-lamp ; the aperture in the piston-rod being open, the air is thus expelled, and when steam freely follows it, the screw may be closed, when, on applying cold to the bulb, as, for instance, putting it on the sùrface of a little mercury in a glass, the included steam is condensed, and a vacuum formed, which causes the descent of the piston, in consequence of the air pressing upon it from above. On again, holding the bulb over the lamp, steam is reproduced, and the piston again forced up, and these alternate motions may be repeatedly performed by the alternate applications of heat and cold. This instrument gives a tolerably correct notion of the application of steam in the old engines, where it was employed conjointly with the air's pressure as a moving power.

In the most perfect construction of Watt's engine, steam is exclusively employed both for elevating and depressing the piston; and the following diagrams and description may perhaps enable the reader to understand the very ingenious method in which it is thus applied:- 


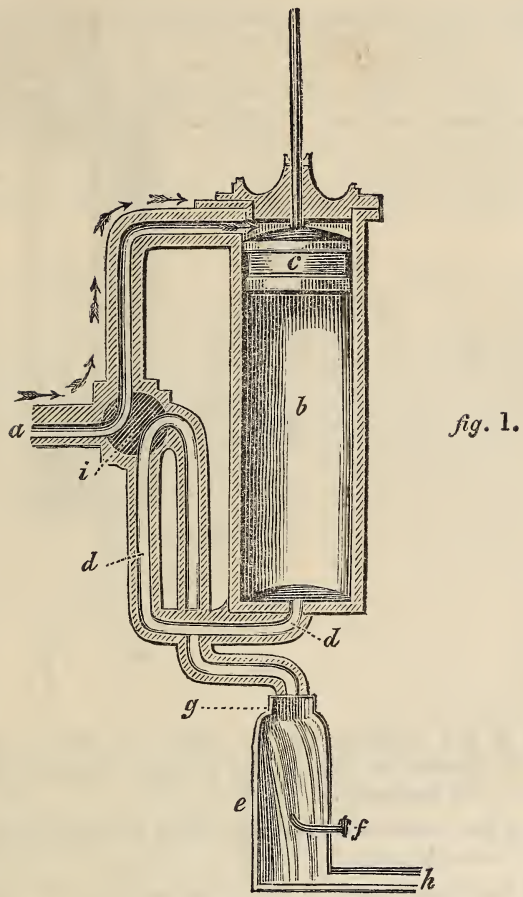

$a$ is the steam-pipe issuing from the boiler, and so contrived as alternately to deliver steam into the cylinder $b$, above and below the piston $c$.

$b$ is the main cylinder of the engine.

$c$ is the piston, with the piston-rod passing through an airtight collar at the top of the cylinder, which is commonly called the stuffing-box. 


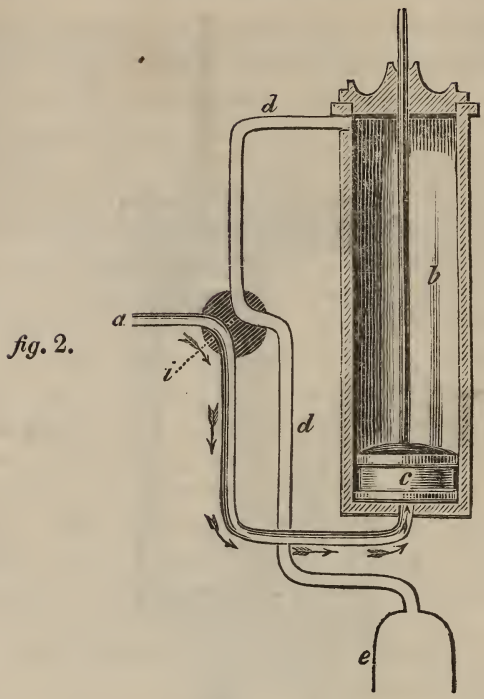

$d$ is the eduction-pipe, by which the steam, having performed its duty in the cylinder, makes its escape into the condenser.

$e$ is the condenser, immersed up to the level $g$, in a cistern of cold water.

$f$, the injection-cock, by which a small jet of cold water is admitted into the condenser.

$h$, a pipe communicating with a pump called the air-pump, by which the condensed steam and injection-water are continually pumped out of the condenser: it also pumps out the air, and keeps a constant vacuum in the cylinder and condenser.

$i$ represents the moveable plug of a cock which has two pas- 
sages, and which opens alternate communications with the steam-pipe and the eduction-pipe. In fig. 1 this plug is shewn in the position which admits the steam to pass into the cylinder above the piston: in fig. 2 the plug has shifted its position, and now admits the steam to pass into the cylinder below the piston : in the former case the condenser is open to the space below the piston; in the latter, to the space above the piston.

Let us now suppose a vacuum to exist in the cylinder $b$; the steam passes through the pipe $a$, in the direction of the arrows (fig. 1.), and entering the cylinder above the piston, immediately forces it downwards. The piston having reached the bottom of the cylinder, the plug of the cock $i$ shifts its position, and is now as represented in fig. 2 ; the steam enters as before at $a$, and passes in the direction of the arrows to the bottom cylinder, so as to elevate the piston. It is obvious that this action could not take place unless the steam by which it had previously been depressed, were removed; this therefore makes its escape by the eduction pipe $d$, ( $f i g .2)$, and passes into the condenser $e$, where it reverts to the state of water, and is got rid of by the air-pump through the pipe $h$ ( $f$ g. 1$)$. The piston being now in the position represented in fig. 1, and the plug having again shifted its position, the steam enters above the piston as before, while the space under the piston has now a free communication with the condenser by the eduction pipe $d$; so that the steam, which had first been active in raising the piston, is not in the way of its depression. I have here represented and described what may, perhaps, be called the simplest form of this engine, 
in order to render its principle as intelligible as diagrams admit. Instead of the four-way plug $i$, sliders or sliding valves are generally used, as being less liable to wear, and more easily kept air-tight; but in all cases the same alternate communication with the boiler and with the condenser is effected.

\section{\$ 13.-Of Radiant Matter.}

The heat which we perceive on standing in the sunshine, and on approaching a fire, or other luminous and heated bodies, is commonly called radiant heat, and is presumed to be produced by the emanation or radiation of a highly attenuated form of matter, from the sun and other bodies.

In the sun's rays this form of heat is intimately blended with light, and their joint effect as chemical agents is extremely interesting and curious. We have above adverted to the influential effects of light upon some of the phenomena of crystallization (page 15); and in other cases they are equally and even more remarkable.

No one can have failed to observe the difference between vegetables thriving in the full enjoyment of solar light, and those which grow in obscure situations, or which are entirely deprived of its agency. The former are of brilliant tints, the latter dingy and white; in the one, the various secretions come to perfection; in the other they are either modified or disappear. Of this, numerous familiar instances might be cited, especially among our esculent vegetables. The shoots of a potato produced in a dark cellar are 
white, straggling, and differently formed from those which the plant exhibits under its usual circumstances of growth. Celery is cultivated for the table by carefully excluding the influence of light upon its stem : this is effected by heaping the soil upon it so as entirely to screen it from the solar rays; but if suffered to grow in the ordinary way, it soon alters its aspect, throws out abundant shoots and leaves, and instead of remaining white and of little taste, acquires a deep green colour, and a peculiarly bitter and nauseous flavour. The interior or heart of endive is not eatable, unless protected from light ; if exposed to it, it becomes disagreeably bitter and tough. The heart of the common cabbage is another illustration. The rosy and coloured aspects of the side of fruits exposed to the sun are referable to the same cause. Changes yet more remarkable have been observed in plants vegetating entirely out of the access of light. In visiting a coal mine, Professor Robinson found a plant with a large white foliage, the form and appearance of which were quite new to him : it was left at the mouth of the pit, when the subterranean leaves died away, and common tansey sprung up from the root.

In the animal creation the influence of light is equally evident. It is curious to remark the dull and dingy tints of polar and subterranean animals, as contrasted with the gaudy and brighter colours of those which inhabit the tropical regions of the globe, and enjoy the full sunshine. In the human species, too, a due quantity of light is requisite to health: miners are generally pallid and unhealthy; and the inhabitants of the dark alleys and courts 
of London indicate a similar want of its beneficial influence; though in these cases bad air and poor diet also contribute to want of health.

\section{$\S$ 14.-Of the Chemical Effects of Radiant Matter.}

The common operations of the laboratory furnish us with some striking illustrations of the influence of light in promoting the chemical agencies of bodies.

There are two gases, called hydrogen and chlorine, which, when mixed and kept in the dark, are nearly without action upon each other; but if exposed to the light of day, they soon act upon each other, and unite to form muriatic acid; and if the sun shines directly upon the bottle containing them, it not unfrequently happens that they inflame and explode in consequence of the energy imparted to their attraction by the solar rays. Some of the salts of gold and of silver are remarkably susceptible tests of the chemical agency of light: the nitrate of silver may be selected by way of illustration. If a piece of paper be dipped into a solution of this salt, and kept in the dark, it suffers no apparent change; but if exposed to light, it soon becomes purple, brown, and black: changes of colour depending upon a chemical change suffered by the salt. A pretty experiment, shewing the action of light upon nitrate of silver, was devised by Mr. Wedgwood : a piece of paper, or other convenient material, was stretched upon a frame and sponged over with a solution of the salt; it was then placed behind a painting upon glass ; and the light, traversing the painting, pro- 
duced a kind of copy of it upon the prepared paper; those parts in which the rays were least intercepted being of the darkest hues.

Scheele was the first to whom the ingenious idea occurred of ascertaining whether all the rays possessed similar chemical powers, or whether they belonged more exclusively to one colour than to another; and he found, upon refracting a beam by the prism into its seven primary coloursnamely, red, orange, yellow, green, blue, violet, and indigo, and throwing them upon a piece of paper prepared with nitrate of silver, that the greatest blackening effect was produced by the violet ray, and that the decomposing or chemical powers of the prismatic spectrum, as this assemblage of colours is called, gradually decreased towards the red ray, where scarcely any effect was produced. This result was quite contrary to expectation; for one would, of course, have anticipated the greatest effect in the most luminous part of the spectrum-namely, about the yellow and green rays.

It was, however, afterwards ascertained that certain invisible rays, occupying a place in the spectrum just beyond the violet extremity, possessed a greater power of effecting the above and other chemical changes, than the violet rays themselves ; and it has, consequently, been inferred that such distinct rays emanate from the sun, possessed, as their place in the spectrum shews, of great refrangibility; and that the coloured rays (and their mixture, constituting white light,) derive their chemical powers from the admixture of these highly refrangible and chemically acting rays, 
which are most abundant at the blue end of the spectrum, and gradually decrease towards the red or least refrangible rays. Thus, it was found that the greatest blackening effect upon the salt of silver was produced just beyond, and out of the violet ray: the other peculiar chemical effects to which we are now adverting were also most manifest in the same spot. It must, however, be observed, that the place occupied by these rays in the spectrum depends, in some measure, upon the nature of the medium by which the light is refracted.

Many years ago, Morichini thought that he had communicated magnetism to steel wires, by exposing them to the rays of violet light. So curious a result attracted much notice, and his experiments were repeated by several eminent philosophers with very various success: it was generally, however, presumed that he was mistaken in his conclusions, and that the needles had acquired magnetism from some other sources. The subject has lately been resumed by Mrs. Somerville, who, from a series of apparently wellconducted experiments, arrived at the same conclusion as Morichini. It must, however, be confessed, that there are many difficulties in the way of attaining perfectly unexceptionable results in these experiments ; and whether magnetism is, or is not, producible by the sole influence of light, is a question not definitively settled. 


\section{\$ 15.-Of the Heating Effects of Radiant Matter.}

It is now time to advert to another peculiarity in the prismatic spectrum which relates to its heating power. If we cause the spectrum to fall upon a sheet of paper, and gradually pass the hand through it, from the violet to the red end, we shall perceive the latter to be sensibly warmer than the former; and, on applying a delicate thermometer in the differently coloured rays, it will be found scarcely affected by the blue; in the green it rises; and in the red, shews an increase of several degrees of temperature.

The observation of this fact suggested the possibility of the heating power of the spectrum extending beyond the red ray, and, on applying a thermometer just out of the red ray, and beyond the limits of the visible spectrum, this was found to be the case. A thermometer in the red ray rose seven degrees in ten minutes, but just beyond the red ray the rise in the same time was nine degrees.

In these, as in the former experiments, the effects are modified by the nature of the prism used to refract the light: when it is of flint glass the phenomena are as above described; but, with crown-glass, water, and other refracting media, the position of the heating or calorific rays varies.

It appears, then, in reference to the results just quoted, that the radiant matter of the sun is resolvable, by refraction, into three distinct sets of rays; those producing colour; those which effect certain chemical changes; and those which excite 
heat; the chemically acting rays are the most refrangible, the calorific rays the least so, and the colorific rays, or those producing light and colour possess a mean degree of refrangibility. In the spectrum obtained by a flint-glass prism, they are, therefore, arranged as follows:-

Colorific Rays.

Calorific

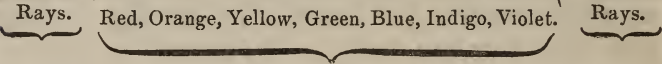

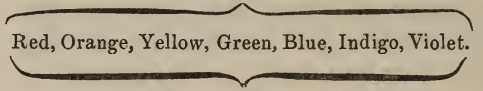

Chemical

$\underbrace{\text { Rays. }} \underbrace{\text { Red, Orange, Yellow, Green, Blue, Indigo, Violet. }}$

$\underbrace{\begin{array}{c}\text { Least } \\ \text { refrangible. }\end{array}}_{\text {Solar Light. }}$

\section{\$16. Of Terrestrial Radiation.}

We use the term terrestrial radiation, to designate the heat and light thrown off by terrestrial bodies under certain circumstances, especially at high temperatures, as when they are red-hot, or burning; that is, in a state of ignition; incandescence; or combustion. We also well know that terrestrial heat radiates from bodies at temperatures far below those required for ignition. If, for instance, I fill a jug with hot water, I feel heat emanating from it to my hand when held near it. All these subjects may be discussed under the head of terrestrial radiant matter.

The rays which emanate from heated bodies, whether attended or unattended by light, may be reflected in the same way as the solar rays, and the phenomena connected with this kind of radiation are, in many respects, extremely curious and important: they are also suspectible of many 
economical applications, for in this country it is to radiant heat that we are chiefly indebted for the warmth and comforts of our apartments in winter; the construction of our grates, and fire-places in general being such as to carry away the whole current of the heated air by the chimney, and prodigally waste it in the atmosphere; they consequently produce their warming effects, by the heat which emanates from the burning fuel and heated surfaces, and is projected or radiated upon the surrounding bodies in the room.

Several important facts upon the subject of the radiation of heat were established many years ago by Scheele (in his treatise on air and fire, originally published in 1777). He observed, that radiant heat passes through air without communicating heat to that medium, and that it is not intercepted by currents in the atmosphere. He then proceeded to ascertain how far it coincided with solar heat, in passing through glass and other transparent media; and he found that, although the light of a fire or of a candle passed through such substances, that their heat was arrested, or stopped in its progress. It was thus thought that a decided difference was established between solar and terrestrial heat; but we shall find that it is rather apparent than real, and referable to the small quantity, or intensity of the latter, as compared with the former. He also found that, when a glass mirror is held before the fire, the light only is reflected, while the heat is absorbed, and the mirror itself becomes hot; but when for glass we substitute a mirror of polished metal, then, both the heat and the light are reflected, and the metal 
itself not heated: if, however, the surface of the metal be covered with some other substance; if it be smoked, for instance, by holding it over a burning candle, then the surface absorbs the rays of heat, and the mirror of metal, like that of glass, becomes itself hot.

These observations of Scheele led Leslie and others into the same path of inquiry, and we have, upon the whole, a very satisfactory series of experiments upon the subject, of which the following is an outline, as far as the principal facts are concerned:-

That heat radiates from bodies in right lines, and is susceptible of reflection, is shewn by placing two polished concave mirrors, made of planished tin, or, what is better, of plated copper, exactly opposite to each other, and about ten feet asunder. For experiments upon a large scale, the mirrors should be at least of two feet diameter, but common tin mirrors or reflectors of one foot are sufficient for all common illustrations. A heated body, such, for instance, as an iron ball, or a flask of boiling water, is placed in the focus of one mirror, and an air thermometer in that of the other*. The rays of heat then impinge on the one mirror, and are reflected, consistently with the property of concave mirrors, in parallel lines, so as to fall upon the opposed mirror, whence they converge

* The foci of the mirrors is best found by the help of a lighted candle, the heating focus corresponding with the luminous one: and in these experiments the differential thermometer above described is most satisfactorily employed, for it is not affected by currents or general changes in the temperature of the room in which the experiments are made. 
to its focus, in which is the thermometer, and which is affected proportionately to the heat of the original radiating body.

Let A represent the first mirror; в the second; c the heated sphere or flask, and $\mathrm{D}$ the air thermometer: the dotted lines shew the direction of the calorific rays.

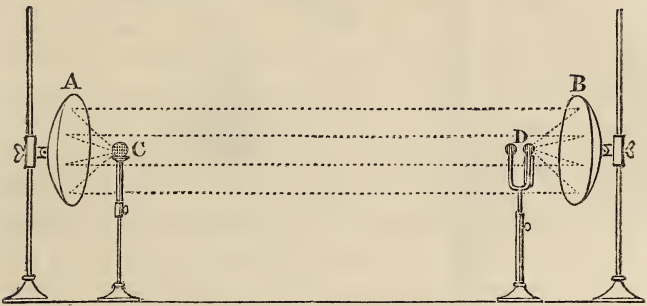

To prove that the thermometer is not affected by the mere proximily of the heated body, independently of any actual reflection, let a sheet of pasteboard be held between the mirror в and the thermometer; the latter will immediately indicate the absence of the quantity of heat it before received, notwithstanding the source is as near to the bulb as it was before. If we even carry the ball a little out of the focus of the mirror $A$, by advancing it gently towards the thermometer, a diminution of heat will be perceived in the latter, in consequence of the disturbance of the most favourable arrangements for reflection. Indeed, the distance between the heated ball and the air thermometer is such, that, notwithstanding the delicacy of the latter instrument, it would not be 
affected, as may again be shewn by removing one or both of the mirrors, and leaving the ball and thermometer in their same relative positions.

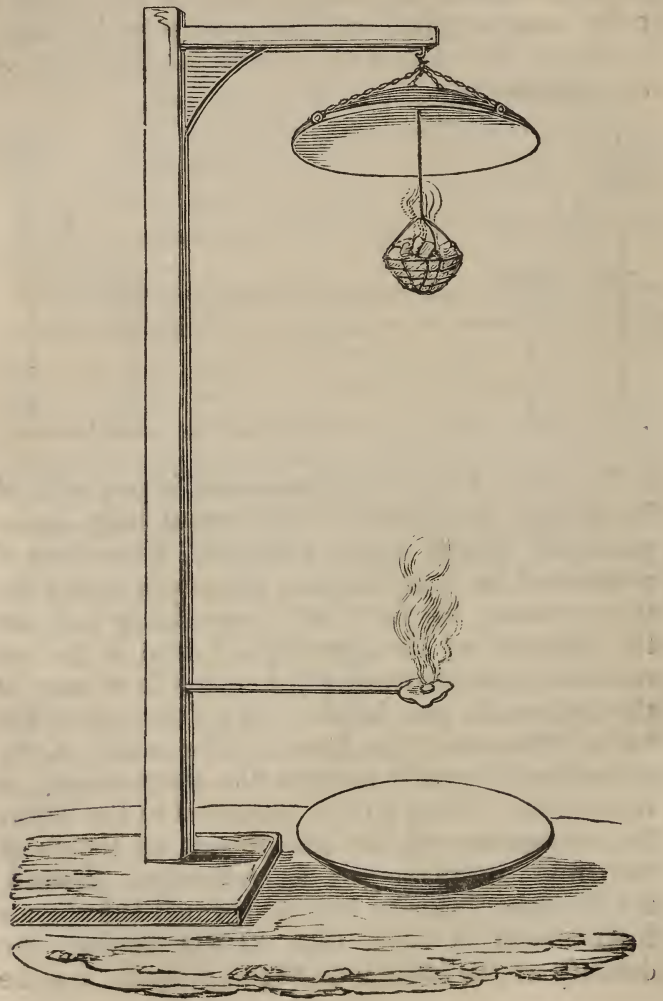


If, for the hot bullet, or flame of a candle, or flask of boiling water, we substitute some more copious source of heat, the effects will be proportionately great. If we, for instance, place a wire basket full of burning charcoal in one focus, and a piece of phosphorus in the other, the latter will be immediately inflamed: in the same way fulminating silver may be detonated, and even gunpowder kindled. And for such experiments we may adopt Sir H. Davy's plan of placing the mirrors vertically, as shewn in the annexed cut; the little chafing-dish is in the focus of the upper mirror, so that all the heat that reaches the lower mirror, and is concentrated in its focus, must be radiant and reflected, for the current of heated air will pass upwards and be lost.

If we substitute a cold body for the heated one, a lump of ice, for instance, sprinkled over with salt, or a thin glass flask, containing a mixture of snow and salt, we shall then observe the thermometer in the opposite focus to be affected by cold; and this experiment has sometimes been adduced to prove that cold is a distinct form of matter. It does, however, seem evident, that highly attenuated matter is at all times passing off from the surfaces of all bodies, under the form, and producing the effects of, radiant matter, the temperature excited by which depends upon that of the emanating body: hot bodies radiate heat: we perceive no effect from bodies at common temperatures, because their radiant matter is of the same temperature as that of the thermometer or substance upon which they fall : cold bodies, on the contrary, throw off cold radiant matter, and hence 
their effect upon the thermometer. That this radiation of cold actually ensues, is more simply shewn by placing a plate of snow or bruised ice near the face, when an effect of cold is immediately perceived, and may be intercepted by a sheet of paper or other screen.

It must, indeed, be obvious, that the temperatures of bodies are merely relative terms: we may, for instance, suppose ourselves in a temperature so high, that red-hot iron would radiate cold; or, again, in a temperature so low, that ice would radiate heat.

Sir H. Davy observes, in reference to this subject, which he calls the apparent radiation of cold, ' that if it be supposed that rays capable of producing heat emanate from all terrestrial bodies, but in quantities greater in some increasing proportion as their temperatures are highest, then the introduction of a cold body into the focus of one mirror, ought to diminish the temperature of a thermometer in the focus of the other, in the same manner as a black body placed in one focus would diminish the quantity of light in the other focus; and the eye is to the rays producing light, a measure similar to that which the thermometer is to rays producing heat.'-Elements, p. 206.

Other explanations have been given of the radiation of cold, but none will be deemed satisfactory, till we are better acquainted with the nature and more recondite properties of radiant matter.

With the preceding apparatus of the mirrors, and a few very simple additions, many other curious properties of radiant matter in general may be investigated; and among these, the infuences of 
surface upon radiation are not the least remarkable.

It has been stated, that if a polished metallic mirror, of a concave or parabolic form, be held opposite the fire, that it will give a heating focus, but that the mirror itself is long before it becomes sensibly warm; that is, the heat which impinges upon it, is immediately thrown off or reflected. If we now cover the surface of the mirror with any soft, unmetallic coating, or if we merely roughen it by scratching the surface with scouring-paper, and then hold it towards the fire, a very different effect will ensue, and we shall now find that the greater part of the radiant heat, instead of being thrown off, is absorbed by the surface, and very soon heats the mirror considerably. A thin paste made with chalk or lampblack, and diluted glue or gumwater, answers very well for the above purpose.

Here, then, the receptive powers of surfaces, in regard to radiant matter, are shewn materially to depend upon their nature or mechanical texture.

In further illustration of this subject, a simple but interesting experiment may be made with the differential air thermometer: let one of the glass bulbs, for instance, be nicely covered with a bright coating of gold-leaf, and leave the other in its usual state: place the instrument thus prepared upon a table, and bring a red-hot poker near it, taking care, however, that it is equally distant from both bulbs, or, if anything, rather nearer the metallic than the glass surface. Under these circumstances it will be found, that by far the greatest effect of heat is produced upon the glass ball, from which the fluid in the tube immediately 
recedes, notwithstanding heat is equally applied to the gilt ball; the latter, however, refuses to absorb it, while the former imbibes it, and has its temperature proportionately augmented.

Although the texture of the surface is shewn to be materially concerned in these results, it does not appear that colour in the least interferes; black-wash and white-wash are equally receptive of terrestrial radiant heat; nor does it appear to be absorbed in larger proportion by any one colour than by another.

With regard to solar radiant heat, however, the case is different, and, as common experience teaches, black surfaces absorb it much more greedily than white, and the temperature produced appears to be proportionate to the intensity of colour.

If pieces of cloth of different colours be exposed to the sunshine upon the surface of snow, the dark colours will sink into it, in consequence of the heat which they produce; the lighter colours scarcely effect the thawing of any portion; and white remains inactive.

The difference between a white and a black hat in heating the head, and between dark and lightcoloured clothing in general, is well known to those who are much exposed to the summer's sun.

Paint the bulb of one thermometer black, and of another white, and expose them to the sunshine; the former will indicate a higher temperature than the latter.

A similar but more striking experiment may be made with the differential thermometer (p.61). Blacken one of the bulbs, and leave the other 
elean and transparent; if the instrument thus prepared be exposed to the sunshine, the excess of heat in the blackened ball is immediately indicated by the recession of the liquid from it to the clean ball. If the thermometer be small and delicate, it is susceptible of the mere impression of daylight, which, slightly augmenting the temperature of the black ball, depresses the fluid in the tube annexed to it. Hence the differential thermometer, thus constructed and applied, has been called a photometer; but it is not capable of affording any correct or useful indications of the comparative intensities of different lights.

In all these cases there appears to be a manifest difference between solar and terrestrial radiant heat; but this is probably rather apparent than real; in the solar beams, radiant heat and light are intimately mixed, and when they fall upon dark surfaces they are absorbed together. The quantity of radiant matter, too, emanating from the sun, is incomparably great in reference to anything which we can produce artificially. The solar beam passes through a thin plate of glass without decomposition, and falling upon black surfaces upon the other side, is absorbed by, and heats them: but terrestrial radiant matter, emanating, for instance, from a lamp, or candle, or fire, suffers, in such case, a kind of decomposition; the light passes through the glass, but the heat is stopped in its progress; in the case of the sun's ray the transmitting glass is not heated; in the other case the glass becomes hot.

But, when it is said that terrestrial heat does not pass through glass, and that glass screens are as 
effectual as opaque ones in keeping from us the heat of the fire, the statement is only partially correct; the most intense artificial heat and light is probably that produced by charcoal ignited by voltaic electricity, and when a small lens is placed before the brilliant star of fire so obtained, and its focus thrown upon the ball of a delicate air thermometer, an elevation of temperature is always indicated.

Numerous other instances might be mentioned, in which the passage of common radiant heat through glass is evident: if we'stand, for intance, upon the outside of a shop-window when a gas-burner within is suddenly turned on or lighted, we perceive the impulse of heat upon the face.

Newton conceived that radiant matter consisted of particles emanating from luminous and heated bodies with prodigious velocity; these particles he imagined might be of different sizes, the smallest, constituting chemical rays and violet light, being the most refrangible; the larger ones forming red light, and heat, and being the least refrangible. Now, it is very possible that the matter emanating from terrestrial bodies may be of a yet coarser texture, or constituted by particles so large as to be arrested by those transparent media which allow a ready passage to solar rays.

Having stated these facts concerning the radiating or emissive powers of surfaces, we may now turn our attention to their absorbing or receptive energies, and we shall find, that those bodies which have their temperatures most easily raised by the action of rays producing heat, are 
likewise those that are most easily cooled by their own radiation; or that, at the same temperature, emit most calorific rays: in other words, the receptive and emissive powers of surfaces, in regard to radiant heat, are co-existent and co-equal.

From what we have said above, it will appear that, if we place two similar metallic vessels filled with water before the fire, the surface of one of the vessels being clean and polished, and that of the other covered with a thin coat of lampblack, the former will receive heat much more slowly than the latter: a blackened saucepan set near a clear fire has its contents much sooner heated than a clean and bright one in the same situation.

Let us now reverse the experiment, and fill the blackened and the polished vessel respectively with hot water, and putting a thermometer into each, leave them upon a table at a distance from each other, and not near the fire. We shall soon observe that the thermometer falls most rapidly in the blackened vessel, which will, in fact, become cool much more rapidly than the other. Perhaps a simpler way of shewing this fact, consists in painting one half of the surface of a convenient tin or pewter vessel with a mixture of lampblack and gum-water, and leaving the other half, or side clean; if it then be filled with boiling water, no very delicate instrument is requisite to prove that heat is thrown off much more quickly from the unmetallic than from the metallic surface; for the hand brought near the former feels a much greater impression of heat than when at an equal distance from the latter.

If we paste writing-paper, linen, or thin woollen 
cloth, or flannel, upon one-half or side of a canister, and leave the other side clean, a very similar effect will be observed, for those surfaces radiate much more than metals; and accordingly, a differential thermometer brought near a canister so prepared, will be affected at a much greater distance by the covered than by the clean surface.

It will be observed, that these effects of radiation are singularly opposed to the conducting powers of the respective surfaces. If we touch the clean part of the canister it burns us; but we may place the finger with impunity upon the paper or flannel, which, though a good radiator, is a comparatively bad conductor of heat.

This subject is further illustrated by the following table of the comparative radiating powers of different substances, drawn up from Mr. Leslie's experiments :-

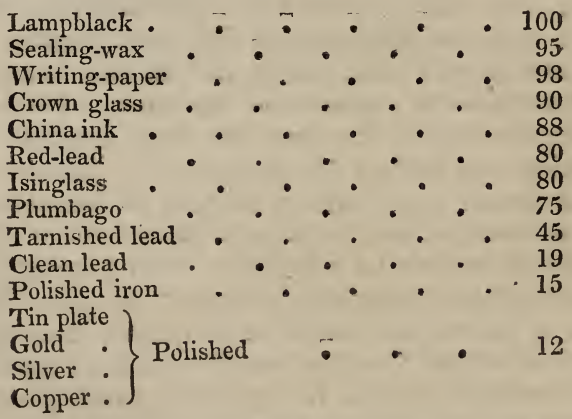

Hence it appears that those surfaces which are not metallic, such as soot, paper, glass, \&c., are 
much better radiators of heat than tarnished metals, lead, or iron ; and that these latter radiate better than the polished metals, which consequently stand at the bottom of the list. It will also be recollected that the above numbers represent the receptive as well as the radiating powers of the respective surfaces.

Let us now look at some of the economical applications of these facts arising out of the philosophy of radiation.

Before much was known respecting the radiating, and consequent cooling power of different surfaces, it was always presumed that vessels of earthenware or porcelain were better calculated to retain heat, than those of metal; it was rightly argued, that the latter were by far the best conductors of heat, and it was inferred, therefore, that they would lose heat to the surrounding air more rapidly than bad conductors. Experienced tea-makers, however, contrary to the decision of such philosophers, always maintained that the best tea was made in a bright silver vessel, and that earthen teapots were of far inferior power; the former were said to draw much better than the latter. This opinion we now know is correct, and the obvious reason for the preference is, that the water retains its heat much longer in the metal than in the porcelain vessel, and consequently extracts the soluble matter of the tea-leaves more completely.

' Vessels,' says Sir H. Davy, 'that are intended to retain their heat should be metallic, and highly polished; and, independent of elegance and delicacy, there is a reason, obvious from the pre- 
ceding facts, why metallic vessels for the purposes of the table should be kept as bright as possible. Steam or air pipes for warming houses should be polished in those parts where the heat is not required to be communicated, and covered with some radiating substance, such as lampblack or plumbago, in those rooms which are to be heated by them. Culinary implements should be blackened, and not polished, on those parts which are to receive heat. The heated surfaces of fireplaces or stoves should not be metallic, but of stony or earthy materials; and in this case much more heat will be communicated by radiation.'

It is also obvious, that whenever radiant heat is to be absorbed, rough, unmetallic surfaces should be employed; and these, in the case of solar rays, may be blackened, in order to render the heat more intense. Brick-walls are excellent absorbers and radiators of heat, and when exposed to the sun's rays they become very hot; afterwards, when the sun goes off, they again radiate the heat which they had previously received, and which caused their excess of temperature over the surrounding bodies. Walls against which fruittrees are planted are sometimes painted black, in order to get the highest possible degree of heat during their exposure to the sun; but it is not found that the crops of fruit are improved by the plan.

The way in which we receive heat from our ordinary fire-places will now be intelligible, and it will be evident that it is principally by radiation, the hot and dilated air making its escape up the chimney, attended by an enormous waste of heat, 
and consequently also of fuel. The heat which radiates from the glowing coals, and from the heated surfaces about the grate, is projected into the chamber, and impinging upon the different bodies around, is received by them with different degrees of facility, dependent upon the nature of their surfaces : the carpet, and all similar surfaces, absorb the radiant heat with facility; furniture and wooden articles become also heated; and these radiate in their turn, and throw off their excess of heat above that of surrounding bodies. They also warm and dilate the air in contact with them, and thus the heat becomes more or less equally diffused throughout the chamber. Our clothes are also, in general, good absorbers of heat; so is the skin or cuticle; and by the absorption of the radiant heat we get warm on standing before the fire, at least upon that side or part which is exposed to it. This partial reception of heat by which we are burned on one side, while the other may be very cold, is one of the great objections to our common mode of warming apartments; and another is, that in winter, one part of the room only is comfortable, whilst others are disagreeably cold. In rooms heated by throwing into them a current of warm air, such inconveniences are not experienced; but then, unless there is at the same time a thorough ventilation, they produce a sensation of closeness which is unpleasant to those accustomed to open chimneys and fires. A combination of the two systems is the most efficacious and pleasant; warm air, properly and judiciously admitted at the parts of the room furthest from the fire; and an open grate, 
to assist in warming, and to accomplish a perfect ventilation. Grates or stoves have lately been contrived, in which there is an open fire as usual, and which also throw out a stream of warm air, heated by being made to pass from below upwards through a double casing, surrounding all the heated parts of the grate: these stoves, when properly made, and applied, are extremely effective and economical.

But it is seldom recollected that all attempts to render any individual apartment in a house uniformly warm, will be frustrated, unless the mass of air in the halls and staircases is also heated : this is most effectually accomplished by a stove upon the basement story, upon Silvester's, or some similar plan; and although the expense of such an arrangement is considerable at first, it will be found, if properly and judiciously made, to effect a considerable annual saving in fuel.

Whilst upon this subject I may be allowed to remark, that it is to be regretted that architects and builders in general are extremely inattentive to the subjects of warming and ventilation, both as concerns dwelling-houses and public edifices.

\section{\$17. Effects of Radiation in Nature.}

The earth, part of the surface of which is daily exposed to the action of the solar beams, will absorb the rays with various degrees of facility depending upon the nature of the surface; and during the night it will again radiate, or throw off a portion of the heat it has so acquired, also with various degrees of velocity. It has been ascer- 
tained that the extent to which this radiation from the earth takes place, is greatly dependent upon the aspect of the sky; when the night is clear, and the heavens cloudless, it goes on more rapidly, and to a much greater extent, than when the sky is overcast and cloudy; and hence it is, that upon clear nights there is a much greater deposition of dew than in cloudy weather. To understand the cause of this, it may be necessary to remind the reader, that dew is not, as often represented, a kind of fine rain showering down upon the earth from above, but that it depends upon the deposition of moisture contained in the state of vapour in the air, and is, in its formation, precisely similar to what happens when a glass of iced water is brought into a warm room in summer; the coldness of its surface abstracts the heat from the vapour in the air, and causes its condensation in the form of water, which is deposited exactly like dew upon the outside of the vessel *. So the earth, upon a fine cloudless night, radiates heat to such an extent as to become colder considerably than the superincumbent atmosphere, and consequently the aqueous vapour, more or less of which is always contained in the air, is condensed in the

* This is also the reason why thick and substantial walls in the interior of houses become damp, and often very wet, in certain states and changes of weather. They are so massive as to retain an acquired temperature for a long: time, and during a winter's frost become cold throughout; upon a sudden thaw, attended by a very humid state of atmosphere, the vapour or moisture of the air is condensed in the liquid state upon these cold surfaces, because they abstract its heat, and consequently cause it to run down in the form of water. 
liquid form upon the surface of the ground; and, of course, most abundantly, upon those parts of the surface, which, being the best radiators, have their temperatures most depressed. Thus, walking in the garden upon a bright autumnal morning after a clear night, we observe the dew very unequally deposited: it is commonly most abundant upon the grass-plats, for mown grass is an excellent radiator; it becomes, therefore, colder than the adjacent gravel-walks, upon which the dew is less abundant. If we take the trouble of placing a thermometer upon the grass, and another upon the gravel, we shall find the former sink several degrees below the latter; and under circumstances favourable to the deposition of the dew, the earth's surface will always be found colder than the air above it. A thermometer on the grass has been observed, under favourable circumstances, to fall as much as from twenty to thirty degrees. below one suspended some feet above it. The consequence of this is, that the lower strata of the atmosphere in contact with the cold surface of the earth, are considerably colder than the higher parts; and if the air is tranquil, it will remain so for some time, especially in hollows, and other places protected from currents, and .where the warm and the cold air are not therefore mixed by mechanical agitation. Hence it is that delicate plants often suffer more in situations apparently protected, than in those which are open and exposed, and where the cold air has not such a chance of lying quiet upon the surface.

All good radiators exposed upon the earth's surface will become colder than bad ones; if, 
therefore, we place upon an exposed gravel-walk, a piece of flannel and a piece of tin foil, the former will have abundance of dew thrown down upon it, and the latter none; and, in reference to this fact, it is curious to observe the influence of the natural textures and surfaces of foliage. Smooth, varnished leaves have no dew deposited upon them, under circumstances in which there is an abundant deposition upon rough and downy leaves ; the former, therefore, seldom suffer from this cause of cold, but the latter are often frostnipped.

The remarkable influence of clouds in preventing radiation from the earth beneath them, seems first to have been noticed by Dr. Wells, whose 'Essay on Dew' deserves the attentive perusal of all who are interested in this part of meteorology. He found that, if the night, at first clear, becomes cloudy, the temperature of the grass always rises, independent of any changes of calmness, and nearly so of change of atmospheric temperature. Upon one such night the grass, after having been twelve degrees colder than the air, became only two degrees colder, the temperature of the atmosphere remaining stationary; and, on another occasion, the temperature of the grass rose fifteen degrees, while the air only sustained an increase of three and a-half.

Any protection interposed between the earth and sky, prevents radiation, in the same way as a cloud. If we place four poles in the ground, and stretch a mat or canvass upon them, the plot underneath will be effectually preserved from those changes of temperature referable to the escape of 
radiant heat from the surface. In this way gardeners protect young and tender plants; though it is obvious that any general changes, dependent merely upon atmospheric temperature, would not be interfered with, nor the cold kept off by such a contrivance.

In the eighty-third volume of the 'Philosophical Transactions,' Mr. Williams has described the mode of obtaining ice in Bengal, where the atmospheric temperature never falls to the freezing point. It is evidently chiefly effected by radiation from the surface of shallow pans of water (and water is an excellent radiator), which are placed upon dry stubble in open situations : calm, serene, and cloudless nights are most favourable to the purpose ; and thin crusts of ice are thus frequently obtained. In such cases the production of cold has sometimes been referred to evaporation, but that this is not the cause of the diminution of temperature, in the present instance, is proved by the peculiar circumstances requisite for the success of the operation being entirely independent of it.

\section{§ 18.-Of Phosphorescence.}

This term is generally applied to those bodies which shine in the dark, without undergoing combustion; they may be divided into three classes :-

1. Bodies which become luminous after having been exposed to light, and which are generally called solar phosphori.

2. Bodies which become luminous when moderately heated.

3. Bodies of vegetable and animal origin, which 
are spontaneously luminous at common temperatures.

1. Solar Phosphori.-The attention of philosophers was first drawn to this curious subject, by an accidental discovery of Vincenzio Cascariolo, a shoemaker of Bologna, who (about the year 1630) being engaged in some alchemical experiments, had occasion to calcine a quantity of a species of native sulphate of barytes, found near Bologna, at Monte Paterno*. He observed, that whenever the spar had been sufficiently heated, it acquired the property of shining in the dark after having been exposed to the sun's rays, and that it would even continue thus to emit light for some hours. In consequence of this discovery, the Bolognian spar came into great request among the curious; and the best mode of preparing the Bolognian phosphorus, as it was called, became a subject of no small pecuniary importance. The most successful preparers of it were a family of the name of Zagoni, who continued to supply large quantities of it to the curious throughout Europe, and retained their monopoly for many years. Their process is not exactly known; but if the spar be powdered, and made into thin cakes, with mucilage of gum tragacanth, and then carefully calcined in the open fire, and suffered to cool slowly, they will be found to answer the purpose: that is, they will glow for some time in a dark room, after having been exposed to a bright sunshine. Some management, which can only be learned by practice, 
is requisite in conducting the calcination. Any variety of sulphate of barytes will answer for the above preparation, but that which is massive, nearly opaque, and of a lamellar fracture, appears, on the whole, to furnish the most luminous product.

There are many other substances possessed of properties analogous to those just described. For our knowledge of one of these we are indebted to Canton, by whose name it is usually known. Canton's phosphorus is made by calcining oystershells in the open fire for half an hour; after which the whitest and largest pieces are selected, mixed with about one-third their weight of flowers of sulphur, pressed into a crucible with a closely luted cover, and heated red-hot for an hour. When the crucible has become quite cold, turn out its contents, and select the whitest pieces for use.

Wilson's phosphorus.-For our knowledge of the following solar phosphorus, which is yet simpler than the preceding, we are indebted to Mr. B. Wilson: "Select a score of oystershells, the thicker they are the better; then take most of the flaming coals, but not all of them, off a fire that is burning briskly, strew the shells over the surface, and replace the coals that have been taken off. In about an hour's time take out the calcined shells, observing to break them as little as possible; and after exposing them for a few minutes to the light, they will be found to have acquired a high degree of phosphorescence, glowing in the dark in a very beautiful manner, with most of the prismatic colours. It is not, however, absolutely necessary 
that the shells should be calcined in the open fire, for if they are heated sufficiently in a close crucible, they will exhibit prismatic colours, chiefly blue and green, though not so bright as by the former method. If the calcination is effected in an iron crucible, all those parts of the shells that have been in contact with the sides of the crucible will glow with a red light. The contact of inflammable matter, and particularly charcoal, with the shells during their calcination, appears eminently to contribute to the brilliancy of the phosphorus. Hence it is that if the shells are calcined in a crucible, in contact with thin plates of steel*, the phosphorus thus produced will be much more bright, and of more various colours than when plates of iron are employed; and, on the other hand, if flat pieces of charcoal are made use of, the intensity of the colours, especially of the blue, green, and red, is far greater than in those produced by the steel.'

Baldwin's phosphorus.-Another solar phosphorus is that discovered by Baldwin. It is made by saturating diluted nitric acid with chalk, and evaporating to dryness, by which a saline product called nitrate of lime is obtained. This, when melted at a dull red heat, cools into a compact mass, which has the property of imbibing and emitting light.

It has been observed, in regard to these solar phosphori in general, that their luminous power is diminished by cold and increased by heat; that they are more brilliant in dry and warm than in

* Steel being a compound of iron and charcoal. 
wet and cold weather; that they shine most intensely after exposure to direct sunshine; but that some of them, especially the oyster-shells, exhibit their phosphorescence when carried into a dark place, after mere exposure to ordinary daylight. It is sometimes said, that they absorb the same coloured light as that to which they are exposed, and that, if exposed to red light, they give out red, to green, green light, and so on; but this appears to be a mistake, the colours of the light being white or reddish white, and often prismatic, but independent of the colours of the rays to which they had been exposed. Moonlight is quite inadequate to render these bodies luminous; but the flash of gunpowder, the light of a bright candle or lamp, or of an electric explosion, suffices to make them shine more or less intensely.

Those which we have enumerated are the most remarkable solar phosphori, but there are several other substances possessed of very similar properties, such as some diamonds, rock-crystal with its surface roughened, certain saline bodies, and writing paper: these, however, are less powerfully luminous, and require good management to be observed. They should be brought out of the light to the observer, who should be stationed in a small closet, painted black on the inside, and with a black curtain hung up before the door.

2. Phosphori from heat.-There are many substances which become luminous when moderately heated; that is, at a temperature below a red heat. These differ from the preceding in the circumstance, that, after having been kept at any particular temperature till their luminousness is ex- 
hausted, they are incapable of shining again, except at a temperature greater than that to which they were first subjected.

The substances belonging to this class are extremely numerous, but there are only a few which are eminently luminous; of these the compact phosphate of lime, found native near Estramadura, in Spain; and certain kinds of fuor, or Derbyshire spar, especially the dark blue and slightly fetid variety, are the most remarkable : the former exhibits a beautiful pale-green light, intense enough to be seen in the daytime; and the latter shines with a purple tint. The best way of exhibiting them is to heat them in a dark room, in a small spoon of platinum, over a spirit-lamp, the light of which is not sufficient materially to interfere with the observance of their phosphorescence. They should be in small fragments, but not too finely powdered. The fetid carbonate of lime, called swinestone, not uncommon near Bristol, several varieties of calcareous spar, and of heavy spar, and powdered quartz, are substances which are also luminous when gently heated; they may be strewed for this purpose upon a piece of iron which has been heated redhot, and just ceased to glow.

The substances which are phosphorescent by heat, are also generally so by friction, which is another productive source of luminosity. There are also several bodies which are rendered lumi nous by passing an electric shock through or over them; such, for instance, as a lump of loafsugar, which shines for several seconds afterwards with a beautiful blue light. 
3. Spontaneously Phosphorescent Substances.Under this head we may place a variety of animal substances which, under certain circumstances, emit light. The flesh of certain fresh and saltwater fish becomes luminous previously to its putrefaction; of these the tench and carp, and the herring and sole, are perhaps the most remarkable: the fish should be gutted and split, and the scales scraped off; if placed in a dark cellar, they are then often observed to shine within twelve to twenty-four hours, the time depending upon the temperature and state of the atmosphere, and probably other causes which have not been determined: this appearance ensues before any bad odour is perceptibly exhaled; and soles are often luminous in the dark when perfectly fit for the table. Lobsters and crabs, under similar circumstances, are often very rich in phosphorescent matter.

Phosphorescence is rarely observed in the flesh of quadrupeds, and never in that of birds under any circumstances of decomposition.

There are many living animals which have various powers of emitting light. The luminosity of the ocean, which is often so beautifully observed in a dark night when the water is more or less agitated, depends upon the presence of various species of insects and marine animals, some of which shine with much brilliancy.

One most familiar instance of a luminous animal is the glow-worm; but there are several others which are not uncommon, and which, under certain circumstances, are powerfully phosphorescent. The common hundred-legged worm, found under 
bricks and pots in the garden, exhibits brilliant flashes of light when irritated. The lantern and fire flies of warmer climates are also remarkable for their emission of light.

In the vegetable world phosphorescence is a less common quality. Decayed wood, and occasionaliy peat, have been observed to emit a faint light; and there are a few flowers, among which may be mentioned the tuberose, which have occasionally been observed to give out brilliant flashes upon a warm summer's evening.

We have now enumerated some of the leading cases of what is properly called phosphorescence; it appears to consist in an emission of light, independent of ignition or combustion, and referable to a variety of causes, none of which have been very satisfactorily investigated or explained. There are numerous other cases of luminosity referable to changes in the electric states of bodies, and which will afterwards be noticed in detail.

\section{\$19.-Of Ignition and Combustion.}

It will be evident, from the contents of the few preceding pages, that there is a connexion between light and heat, and that they are mutually disposed to produce each other ; indeed, they are probably convertible into each other; but these, and other mysterious relations between them, are as yet very imperfectly understood. I shall not, therefore, trouble the reader with the various hypotheses which have at different times been sent forth in relation to this subject, and which are all unsatisfactory, but shall proceed to notice some further effects of heat. 
In the mean time it may be right to mention a supposed exception to the relationship between heat and light, furnished by an examination of the lunar rays, which, though concentrated by the most powerful lenses, are incapable of affecting very delicate thermometers. De la Hire collected the rays of the full moon when in the meridian, by a lens thirty-five inches in diameter, and threw them upon the bulb of a very sensible air thermometer, but they produced no effect, though they were thus concentrated 306 times.

It is not, however, surprising that the moonbeam should be thus inefficient, when we consider the extreme feebleness of its illuminating power, as compared with a solar ray of the same size; the light of the latter being at least 300,000 times greater than that of the former. It appears probable, therefore, that the properties of the moonbeam are to be referred to its feebleness, as compared with that of the sun, rather than to any peculiarity in its nature, or to any power in the surface of the moon of retaining the heat, whilst it emits the light received from the sun.

By the terms ignition, and incandescence, we express a property which bodies possess of giving out light, whenever their temperatures are raised up to a certain high point. The quantity of light thus emitted is always observed to increase with their temperature : at first it is dim and feeble; then it becomes dingy red, and the bodies are said, in common language, to be red-hot; then bright red, commonly called a cherry-red heat; then, as the temperature increases, the body becomes of an orange or yellow tint, and at length acquires 
such brilliancy as to be painful to the eye; the latter is usually termed white heat.

Various experiments have been instituted to determine the temperature at which bodies become visibly red-hot; this, in a dark place, certainly exceeds six hundred and sixty degrees of Fahrenheit's scale, for at that temperature mercury boils, and it is not, in the least degree, luminous. Sir H. Davy's experiments place the degree of incipient luminosity in a dark place at about $810^{\circ}$. A dull red, visible in daylight, is probably equal to about $1000^{\circ}$, a full red heat to $1200^{\circ}$, and an orange heat to $1650^{\circ}$.

These observations of course only apply to bodies capable of sustaining high temperatures without changing their state, or undergoing decomposition, most substances being either dissipated or destroyed before they attain the requisite temperature for ignition; or, if heated in the air, infiaming and undergoing rapid combustion.

In all the ordinary cases of combustion air is present, and the phenomena presented by the burning body are referable to the chemical action which ensues, at high temperatures, between it and one of the component parts of the air, called oxygen, which, being invisible, long escaped observation as an important agent in the process.

The temperatures at which bodies burn or inflame in the air are extremely various; some take fire under all circumstances the moment they are exposed to it ; others require their temperatures to be more or less elevated; and some refuse to burn under any circumstances. Thus we speak of combustible and incombustible, or inflam- 
mable and uninflammable bodies; and we call the oxygen of the atmosphere, and such other bodies as are possessed of similar powers, supporters of combustion. All common cases of combustion, then, taking place in the atmosphere, depend upon the union of the combustible with oxygen; between these bodies there necessarily exists a strong chemical attraction, and of this, one of the results, as we have already shewn, is the evolution of heat and light. (See page 24.)

The result of the union of the combustible with the supporter, is called the product of combustion; it is very commonly gas or vapour, and eludes ordinary observation: thus the immense quantity of coal daily consumed as common fuel, combines with the oxygen of the air, and forms several products, of which the principal are carbonic acid gas and steam; and these pass off by the chimney, and are dissipated in an invisible state through the immense mass of the atmosphere. The incombustible earthy matter contained in the coal resists combustion, and forms ashes; or mixing with a part of the coal, renders it difficult of combustion, as we see in cinders.

But the product of combustion is often a solid body: thus, if we throw a piece of the metal zinc into the fire, or heat it red-hot in a crucible, it burns with a very vivid flame, and a quantity of a solid white substance is produced, which is a compound of the metal and the oxygen of the air, and is, therefore, called oxide of zinc.

Nothing is more common than to speak of the destruction of bodies by combustion; but the 
term is in so far improper, inasmuch as matter is indestructible; and when we seem to destroy a coal by burning it, we, in fact, only cause it to enter into new combinations; and the carbon which it chiefly consists of, although it apparently disappears, has, in fact, only entered into a new combination, which may be found escaping from the chimney in the invisible state of air or gas, but from which the solid carbon may again be obtained by certain chemical operations.

So much, then, for the general results of combustion; it is often, as we have had occasion to state, accompanied by flame, the nature of which has lately been studied with much attention and curious results by several eminent chemists.

It has often been doubted whether pure gaseous matter is, under any circumstances, susceptible of becoming luminous; certain it is, that its temperature may be elevated far beyond that which is required to ignite solid bodies, and it yet remains invisible. By passing air over heated surfaces, its temperature has been so far elevated as to render metallic wires red-hot, yet was the air itself not at all luminous. Flame, however, may, in general, be regarded as luminous gaseous matter, and its temperature is, under certain circumstances, very intense, even where the light which it emits is excessively feeble. Perhaps the purest form of flame which we can exhibit is that of hydrogen gas*; it is so feeble as scarcely to be

* The term hydrogen implies generator of water, for when it is burned in the air it unites with oxygen, and produces water; water, therefore, is a compound of hydrogen and oxygen, in which, as we shall afterwards more fully 
visible in broad daylight; yet its temperature is very high, as may be shewn by holding a fine platinum wire in it, which immediately becomes white-hot: the other metals, under similar circumstances, are generally melted or burned.

shew, one part of hydrogen unites to eight of oxygen to produce nine of water (see p. 31). By decomposition, water

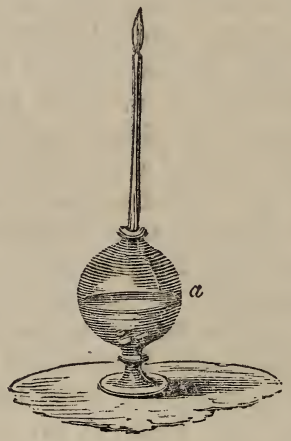

may be resolved into hydrogen and oxygen gases in the above proportions.

In experiments where the fame of hydrogen is required, the following simple apparatus may be used: $a$ is a globular bottle, into which we put some pieces of bruised zinc, and pour upon it a mixture of one part of sulphuric acid with eight of water, so as about half to fill the vessel; it is stopped with a cork perforated by a metallic or glass tube which is drawn to a fine aperture. The ingredients in the bottle soon begin to act upon each other, effervescence ensues, and hydrogen is rapidly liberated; after it has escaped by the tube for some minutes, and not before, apply a light to it, and it will be kindled, and burn with a very pale flame. 
Upon what, then, does the luminosity of a flame, which renders it valuable as a source of artificial light, depend? Principally upon solid matter diffused through it, and ignited by it. In the experiment just cited, the flame of the hydrogen is rendered luminous by the wire; and if we blow any substance through it in fine dust, its brightness will be proportionately increased, though the solid matter be not itself inflammable; as, for instance, if we sift a little magnesia into or through it.

In the flame of tallow, wax, oil, and coalgas, the brilliancy of the light is chiefly owing to finely-divided charcoal, which is blended with, and burned in, the flame, and which, if in too large quantity, causes the flame to smoke, as we see especially in ill made tallow candles, which, notwithstanding frequent snuffing, throw off a quantity of soot into the surrounding atmosphere, creating a disagreeable suffocating smell, and blackening the walls and ceilings of our apartments.

Such, then, appears, to be the source of the light of flames; but there are some other curious particulars respecting their construction, which require more full explanation.

In a common candle, the wax or tallow is drawn into the burning wick by capillary attraction, and there is converted into vapour, which ascends in the form of a conical column, and has its temperature sufficiently elevated to cause it to combine with the oxygen of the surrounding atmosphere with a temperature equivalent to a white heat. But this combustion is superficial only, the flame being a 
thin film of white-hot vapour, enclosing an interior portion, which cannot burn for want of oxygen. That this is the case is easily shewn, by bringing down a piece of thin glass upon the flame of a candle, so as to cause a transverse section of the flame. We shall then observe a ring of light surrounding the interior dark part of the cone, as in the

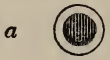

annexed sketch $a$, and the vertical section of the flame will put on an appearance resembling the following figure $b$. To prove that the interior

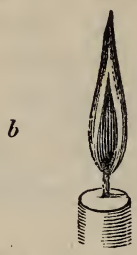

dark part consists of inflammable gaseous matter, we may insert one end of a small glass tube into the dark centrical portion of the flame of a large candle, (as shewn in the wood-cut at the head of the opposite page), and the interior gas will then make its escape through it, and may be lighted at the other end of the tube, so as to form a second flame. $a$ shews a section of the flame; $b$ the glass tube held in an inclined position in the central part of the flame; $c$ the inflammable gas kindled at its extremity. 


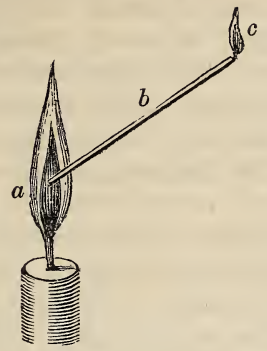

There are several vapours which burn at lower temperatures than those required for the production of flame; and the phenomena attendant upon such cases of combustion are extremely curious. When spirit of wine, for instance, is burned with flame in the usual way, carbonic acid and water are the products of its combustion; but when burned without flame, it produces a peculiar modification of acetic acid or vinegar.

The mode of effecting this slow combustion is sufficiently simple. A small spiral coil of fine platinum wire is placed upon the wick of

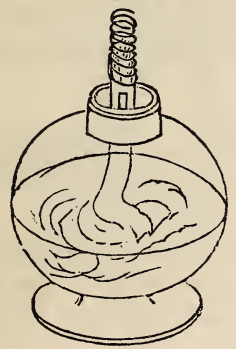


a spirit-lamp, and allowed to project about a fourth of an inch above it, as represented in the last wood-cut. The lamp is then lighted, and suffered to burn for a few seconds, when the flame is put out by an extinguisher, which, however, must be instantaneously removed. In this way the coil of wire retains heat enough to carry on the slow combustion of the alcoholic vapour, and it continues to glow with a red heat as long as spirit remains in the lamp, and to produce the peculiar acetic product just mentioned: in this way of proceeding, the heat never becomes intense enough to cause the inflammable vapour to burn with flame.

There is another, and a very instructive way of making this experiment, which consists in pouring a teaspoonful of ether into a small glass jar of the form represented in the following cut, and then suspending in it a coil of fine platinum wire,

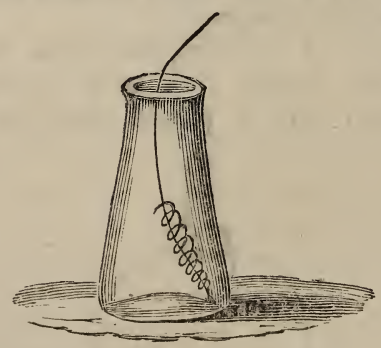

previously heated by holding it for a minute in the flame of a spirit-lamp. The wire being now immersed nearly red-hot into the mixture of at- 
mospheric air and etherial vapour, glows with a red heat, causing the slow combustion of the vapour. It often happens that a part of the wire becomes white-hot, and in that case flame is produced, and the ether burns with its usual products ; but so long as the wire continues only red-hot no inflammation ensues.

It follows, then, that flame requires a very high temperature for its existence; and, consequently, that if we can devise any mode of cooling it, it will be extinguished. A very small flame, for instance, is extinguished by bringing a large mass of iron near it, or by carefully surrounding it by a coil of wire; the metal abstracts its heat.

But by far the most interesting experiments upon this subject are made by help of brass or iron wire-gauze, which may easily be obtained of different degrees of fineness, and with which the following illustrative experiments are easily made.

Procure a piece of this gauze about nine inches square, and of such fineness as to have about thirty meshes in the square inch: bring it gradually down upon the flame of a wax candle, or, what answers better in all these cases, upon a jet of coal gas, and the appearances represented below will be produced. The flame will be cut

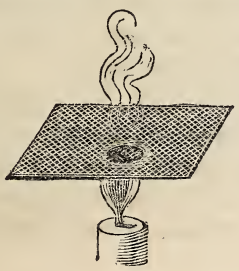


off where it touches the gauze, and we have a good opportunity of observing the exterior luminous circle described above (page 152). In this case the inflammable matter of the flame (in the form of smoke) passes through the wiregauze ; but, in its passage, it is so far cooled as to be extinguished, and the flame is thus prevented from traversing the wire-gauze. If we now bring a lighted taper above the flame, to the upper side of the wire-gauze, the gas or smoke will be kindled, and the flame continue to burn uninterruptedly, thus, -

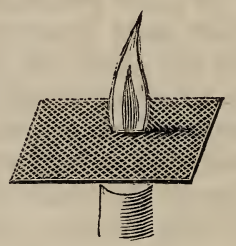

$\therefore$ If the meshes of the wire-gauze used in these experiments be not sufficiently small, or if the wire becomes intensely heated, the flame will pass in either instance; because the cooling power is in the one case prevented by the largeness of the apertures, and, in the other, by the high temperature of the wire.

This experiment may be curiously varied by placing a small piece of camphor in the centre of a piece of wire-gauze about a foot square, and applying a light to its lower surface. The vapour of the camphor, which is very inflammable, burns 
with a bright flame upon the lower surface of the gauze, but cannot pass upwards through it, in consequence of its cooling power; so that the camphor itself lies upon the surface of the gauze, in an uninflamed state, though sufficiently heated to furnish inflammable vapour for the sustenance of the flame beneath*.

These, and many other interesting investigations connected with the subject of flame, we owe to Sir H. Davy, who most ingeniously applied them in the construction of his miner's safetylamp.

Coal mines are often infested by a species of inflammable gas (carburetted hydrogen), known under the name of fire-damp. When this gas is mingled in certain proportions with atmospheric air, it forms a mixture which kindles upon the contact of a lighted candle, and often explodes with tremendous violence, killing the men and horses, and projecting much of the contents of the mine through the shafts or apertures, like an enormous piece of artillery. Many attempts had been made at different times to light the mines

* The power of a metallic tissue thus to intercept or extinguish flame, will depend upon the heat required to produce the combustion, as compared with that acquired by the tissue ; and the flame of the most inflammable substances, and of those that produce most heat in combustion, will pass through a metallic tissue that will intercept the flame of less inflammable substances, or those that produce little heat in combustion: so that different flames will pass through at different degrees of temperature. The general cooling power of the wire-gauze, is referable to its excellent conducting power for heat; it also, by use, becomes a very powerful radiator of heat. $i$ is 
subject to this fire-damp in such a way as to avoid the risk of inflaming it, but none were effectual; at length Sir H. Davy constructed a lamp upon the principles which we have just explained, and which has been used with safety and success.

It is obvious, that if we place a lighted lamp or candle within a perfect cage of wire-gauze, no flame will be able to penetrate from within to the surrounding medium, in consequence of the cooling power of the metallic tissue. The following wood-cut is a representation of a safety-lamp con-

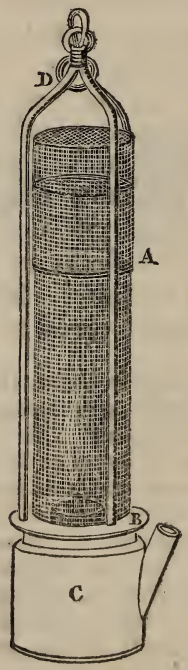


structed upon such principles. A is a cylinder of wire-gauze, with a double top, securely and carefully fastened, by doubling over, to the brass rim B, which screws on to the lamp c. The whole is protected and rendered convenient for carrying, by the frame and ring $D$. If the cylinder be of twilled wire-gauze, the wire should be at least of the thickness of one-fortieth of an inch, and of iron or copper, and 30 in the warp, and 16 or 18 in the weft to the square inch. If of plain wiregauze, the wire should not be less than one-sixtieth of an inch in thickness, and from 28 to 30 both warp and woof.-Davy on the Safety-lamp, p. 114, et seq.

The operation of this lamp may be shown on a small scale, by suspending it in a glass jar, and then admitting a sufficient stream of coal gas to render the inclosed atmosphere explosive; or, what answers nearly as well, by immersing it into a jar, at the bottom of which is some sulphuric ether, and which supplies, by its vapour, an inflammable atmosphere. The flame of the lamp first enlarges, and is then extinguished, the whole of the cage being filled with a lambent blue light; on turning off the supply of gas, or gently blowing the etherial vapour out of the vessel, with a tube or bellows, this appearance gradually ceases, and the wick becomes rekindled when the atmosphere returns to its natural state.

As the safety of these lamps entirely depends upon the perfect state of the wire-gauze, and upon the non-existence of any aperture or channel sufficiently large to admit of the passage of flame, they should, when in use in a coal mine, be inspected 
daily, to insure their soundness in these respects. In gas manufactories, spirit warehouses, and in all cases where inflammable vapours or gases are likely to be generated, as in the examination of foul sewers and drains, it is obvious that these lamps are importantly applicable. 


\section{CHAPTER III.}

ELECTRICITY-ITS SOURCES AND EFHECTS.

MANY of the phenomena of electricity are of so striking and remarkable a nature, and so easily exhibited, as long to have attracted general attention: by the natural philosopher they have been studied with much precision and success; and, more lately, the discovery of the influence of the electrical over the chemical powers of matter, has opened an entirely new field of research.

The object of the following pages will be, to give such general and particular details connected with this subject as are requisite to elucidate its chemical agencies. Of the nature or cause of electrical appearances we are utterly ignorant; but, for the convenience of description and discussion, it has been customary to refer them to the presence of a highly attenuated form of matter, which has been termed the electric fuid. It must always be remembered, however, that the existence of such a fluid, or of any peculiar form of matter as productive of electrical phenomena, is entirely hypothetical, and merely assumed to facilitate our reasoning upon the subject.

§ 1.-Of Electrical Excitation-Attraction and Repulsion.

The simplest indication of electricity is that which is observed when certain resinous and vitreous 
bodies are subjected in a dry and warm state to gentle friction ; as when a piece of sealing-wax is rubbed with flannel, or a glass rod with a piece of silk: in these cases the sealing-wax and the glass acquire the property of attracting and repelling light substances; and they are said to be electrically excited. Such properties seem first to have been observed in, and supposed peculiar to, amber; and hence the term electricity, from the Greek word elektron, signifying amber.

Independent of these attractive and repulsive powers, there are other equally curious properties observed in the electrically-excited bodies; in the dark they appear luminous, shining, whilst rubbed, with flashes of a bluish light; and, if held near the face, they produce a singular tickling sensation.

A very light downy feather, perfectly clean and dry, and suspended in any convenient way by a long thread of white sewing silk, so as to be as independent as possible of surrounding bodies, is a most delicate and useful indicator of electrical excitation, and may be used in the following experiments.

1. Provide a glass tube, about three feet long, and three-fourths of an inch diameter, and having wiped it perfectly clean and dry, rub it gently with a warm silk handkerchief; it will become electrically excited; and, on approaching the feather, will immediately attract it; but, on gently withdrawing, and again bringing it near to the feather, the feather will be steadily repelled; so that it will be impossible, for a short time, to get it to touch the tube: after a while, however, it again 
flies to, or is attracted by, the tube, and then again repelled as before. (The annexed wood-cut represents the appearance of the feather when attracted and when repelled).
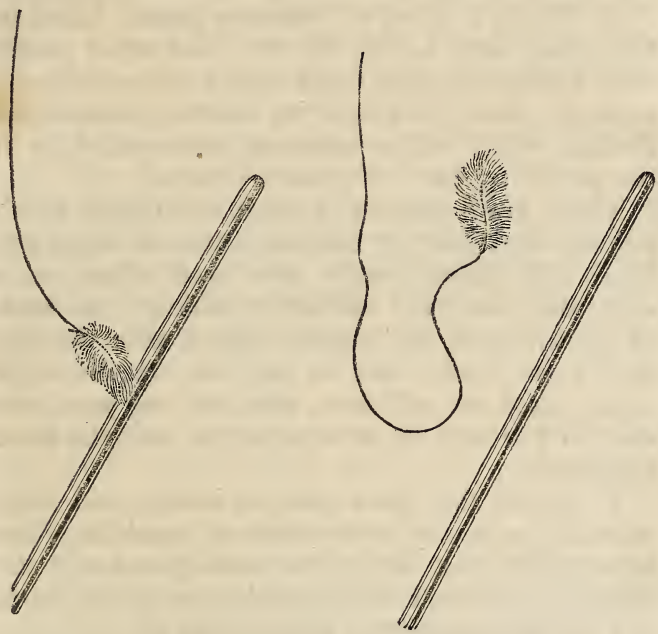

This apparently trifling experiment teaches us the following important elementary fact : it shews us, that the feather, having received electricity from the glass by direct contact, is repelled by it; and, consequently, that bodies similarly electrified repel each other.

2. Rub a large stick of red sealing-wax with a thick roll of dry and warm flannel; the sealingwax will thus be rendered electrical, and will act 
upon the feather apparently in the same way as the glass ; that is, first attracting and then repelling it; and the moment that the feather has become saturated with the electricity of the wax, those bodies become mutually repulsive.

3. Warm a sheet of foolscap paper, folded in the usual way, before the fire, and rub it gently with a large piece of India rubber; then suddenly open the sheet, and hold its interior towards the feather, which will be attracted and repelled, as in the preceding cases of glass and wax.

There are a number of other substances which become electrical by friction, such as white and black silk drawn gently over each other, as is commonly observed with silk stockings; the back of a clean black cat gently rubbed with the dry and warm hand; and so on: but the instances above cited are sufficient, and we may now proceed to a second set of experiments with the same apparatus.

1. Excite the glass tube as before, and electrise the feather, so as to render it repulsive of the tube ; then bring the excited sealing-wax near the feather, and it will not be repelled as by the glass, but will be immediately attracted by it.

2. Reverse the experiment, and having electrised the feather by sealing-wax, bring the excited glass rod near it, when, instead of being further repelled, it will be attracted.

These experiments shew us that there is a difference in the electricities of the glass and sealing-wax, and that bodies rendered repulsive by glass are attracted by wax; and vice versâ. We had previously found that bodies similarly electri- 
fied repel each other; we now observe that bodies dissimilarly electrified attract each other.

These dissimilar electricities, in reference especially to the above methods of producing them, were originally termed vitreous and resinous; for these, the terms positive and negative are now more commonly substituted: the feather electrised by glass is said to be in a positive state, and that by sealing-wax, negative.

Still retaining the simple apparatus that we set out with, we may now proceed as follows:

1. Fold the silk in such a way as to keep the hand as far as possible from the glass tube; then rub the tube with it, and electrise the feather, which will now be repelled; whilst it retains this repulsive state, approach it with the part of the silk which had rubbed the glass, and it will be found to be attracted.

From this experiment we should conclude that the silk and the glass were in opposite electric states : we have already called the glass positive; the silk, therefore, must be negative.

The experiment may be varied as follows :-

2. Hold the handkerchief as before, and having warmed it, rub it with the tube; then bring it near the feather, which it will first attract and then repel: whilst the feather is thus repelled by the silk, approach it with the excited glass, by which it will be immediately attracted. It is obvious, therefore, that the glass and the silk are in opposite states.

3. If we roll up the flannel so as to keep the rubbed part at a distance from the hand, then rub it with the sealing-wax, and bring it to the 
feather, the latter will be immediately attracted by the flannel, and then repelled: if we now approach it with the excited wax, instead of being more powerfully repelled, it will be instantly attracted. Hence, having agreed to call the electricity of the wax negative, that of the flannel must of course be positive; for it is evidently of the opposite or dissimilar kind.

Here, then, we have developed another important electrical law, shewing that one kind of electricity cannot be produced without the other; that when a body is positive, some part of the exciting arrangement is negative. In the above case of glass and silk, the former is positive, and the latter negative; and in the case of sealingwax rubbed by flannel, the wax is negative, and the rubber acquires a positive state.

4. That this is so, may be further shewn as follows:-render the feather positive by the contact of the excited glass; then approach it with the excited flannel, and it will be found to be repulsive of the flannel; that is, to possess a similar electrical state.

5. Electrise the feather with the excited wax, which will render it negative, and then approach it with the excited silk; the silk will continue to repel the feather, and hence is similarly electrised, or negative.

Delicate instruments shew us, that in almost all cases of friction, under the precautions above described, bodies become electrically excited; and that the same body becomes either positive or negative, according to the circumstances under which it is excited. Smooth glass, for instance, 
becomes positive when rubbed with a piece of woollen cloth, but rough glass rubbed in the same way becomes negative; and sealing-wax, which becomes negative when rubbed by flannel, becomes positive when rubbed against tin foil or mercury. A cat's back is said always to exhibit positive electricity; but it is probable that no peculiar kind of electricity is inherent in any one body.

\section{§ 2.-Of Electrical Conductors and Non-Conductors.}

There are some substances which, when examined by the above-described methods, are not observed to become electrical. If we, for instance, rub a cylinder of metal with flannel or silk, it is perfectly inert when brought near the feather: but the exception here is apparent only; the substances previously used, such as glass and wax, are non-conductors of electricity; that is, they receive and retain it upon the excited surface only; but a bar of metal is a conductor, and when electricity is produced upon it by friction, it immediately spreads over its whole surface, and is car. ried off, as it were, by the hand, to the earth and all surrounding conductors. To shew that the metal actually is excited in the same way as glass, attach it to some non-conductor: for instance, attach a cylinder or plate of brass to a rod of sealing-wax, which may serve as its handle; then rub the brass against a dry and warm flannel, and, taking care not to touch any part of it with the hand, bring it near the feather, which will be attracted, and then repelled, precisely as by the 
glass and wax. In these cases the metals usually acquire negative electricity.

We now, then, can understand the division of bodies into conductors and non-conductors. There are also a set of bodies intermediate between the above extremes, which are called imperfect conductors. The nonconductors, readily exhibiting their electricity by friction, are often called electrics, in opposition to the metals, and good conductors, which are called nonelectrics. The electrics, from their power of preventing the passage or transfer and escape of electricity, are also sometimes termed insulators. Thus, a brass rod mounted upon a stem or foot of glass or sealing-wax, is said to be an insulated conductor.

The metals are by far the most perfect conductors ; next to them come well-burned charcoal and plumbago; then some of the concentrated acids, and strong saline solutions. Water, rarefied air, most vapours, and many stony bodies and metallic ores, are imperfect conductors.

Shell lac is the most perfect non-conductor, and an excellent substance to use wherever good insulation is required. Sulphur and wax are also non-conductors; and so is glass, which, though in this respect much inferior to some other substances, is, from the convenience of its application, the usual insulator employed in all common electrical apparatus. Raw and bleached silk stand high upon the list of non-conducting substances ; but dyed silk is often a partial conductor, in consequence of the substances used to colour it: hence, in the above experiments, we have advised 
white silk for the suspension and insulation of the feather. Dry air, and baked wood, are nonconductors.

There is no constant relation between the states of bodies and their conducting powers. Among solids, for instance, metals are conductors ; resins non-conductors: among liquids, strong alkaline and acid solutions are good conductors ; water is an imperfect conductor; oils are non-conductors. Solid wax is almost a non-conductor, but when melted it becomes a good one. Conducting powers belong to bodies in the most opposite states ; thus, the flame of alcohol at a white heat, and ice at the temperature of $32^{\circ}$, are conductors. Glass, when cold, is a non-conductor ; but when red-hot it is a conductor: the diamond is a nonconductor: but pure and well-burned charcoal, which is merely another form of diamond, is among the best non-metallic conductors.

\section{§ 3.-Of Induced Electricity.}

We have laid it down as a general rule, that no electrical attractions exist, except between bodies in opposite or dissimilar electrical states; and we have shewn that when any electrified body, such, for instance, as an excited stick of sealing-wax, is brought near other non-electrified bodies, they have a tendency to be attracted by it. This tendency exists among all surrounding substances, but such, of course, only as have freedom of motion, are observed to move towards the excited electric: such as the suspended feather, particles of dust, and any other light and moveable bodies. 
Now, as these are attracted by the electrified body, it follows that they must previously be thrown into an opposite electric state, by the mere proximity of the excited electric; and this is really the case: whenever an electrified body is brought near to another in an unelectrified state, the surface of the latter, opposed to the former, becomes oppositely electrical, and is consequently attracted by it.

Electricity is thus said to be induced in the surrounding bodies, by the vicinity of any excited electric: thus, when a highly electric cloud hovers over the earth, that portion of the surface opposed to the cloud, becomes in an oppositely electrical state, and consequently attractive of the cloud ; thus, during a thunder-storm, we often see an electric cloud perch upon the summit of a hill, and remain there till it has discharged its electricity, when it is wafted quietly away.

Electricity by induction presents us with some very curious phenomena, which may here be stated generally, and more particularly explained afterwards. We have already said, that when an electrified body approaches another in its ordinary state, the surface of the latter opposed to the electrified body acquires an opposite state: this opposite state, however, only belongs to the opposed surface; for the other side of the body, though also electric, is dissimilarly so. This statement may be rendered more intelligible by the following dia gram:- 


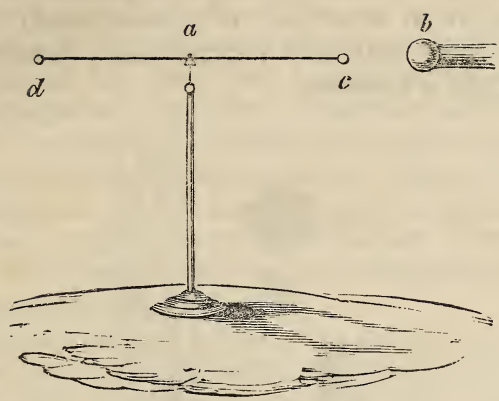

Let $a$ represent a brass needle freely moving upon its centre, which is supported upon a glass foot. $b$, a conductor positively electrified, and within a few inches of the pith ball $c$ attached to one of the points of the needle. We shall now observe that $c$ is attracted by $b$, and may therefore infer that it is negative; and that this is really the case may be shewn by bringing near to the ball $c$, a stick of excited sealing-wax, by which it will be repelled. But if we now bring the sealing-wax towards the ball $d$, it will be attracted by it: here, then, by the proximity of the conductor $b$, we have induced in the needle $a$ an electropolar state; $b$ being positive, $c$ is negative, $d$ positive. To explain this further by the help of our hypothesis : let us suppose the natural or quiescent electricity of $a$ to be disturbed by the presence of $b$, which causes a portion of it to be repelled or driven from $c$ to $d$. The end $c$, therefore, is negative, or minus, that is, it has less than its natural 
portion of electricity; and $d$ is positive, or plus, because in it electricity is accumulated: in the centre there will be a neutral point, in which no electricity is manifest.

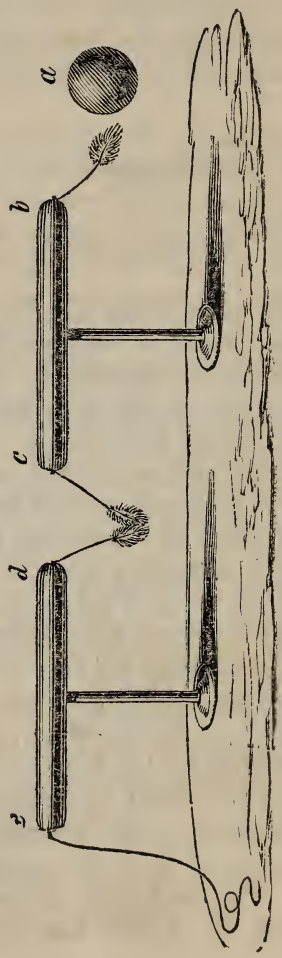


The polar arrangement thus caused by induced electricity, may be carried to any extent: thus, suppose $a$ in the preceding diagram, to represent a body negatively electrified, $b$ would become positive, $c$ negative, $d$ positive, $e$ negative, and so on, through any extent of conductors, the last being in communication with the earth. Thus the feather attached to the insulated conductor at $b$, is attracted towards $a$, and the feather $c$ attracted by $d$; these feathers and surfaces being respectively in oppositely electrical states.

Having now described the properties which bodies acquire by electrical excitation, we may next advert to some instruments and apparatus requisite for their further examination.

\section{§ 4.-Of Electroscopes and Electrometers.}

Electroscopes and electrometers are instruments by which any change in the electrical states of bodies are rendered evident, and their intensities measured.

One of the simplest of these consists of two small pith balls, suspended by very fine thread, or

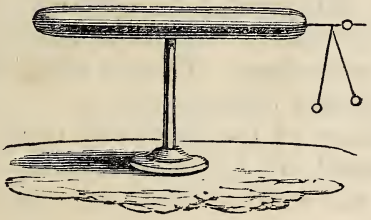

silver wire, to the end of a small insulated conductor. When this receives electricity, the balls 
diverge, and the nature of the electricity by which they are diverged may be judged of, by approaching the conductor with a piece of excited sealingwax: if the divergence increases, it is the same as that of the wax, or negative; if it diminishes, it is opposite to that of the wax, or positive.

Where a more delicate test of the presence of electricity is required, we substitute for the pith balls, two small slips of gold leaf; and as these are apt to be deranged by the slightest motion of the air, it is necessary to enclose them in a glass cylinder, sufficiently capacious to allow of their

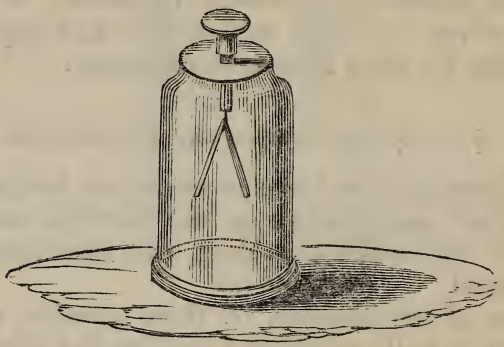

divergence. They are connected with the brass cap of the instrument, which is used in the same way as the preceding. This is commonly called Bennet's gold-leaf electrometer.

The delicacy of the gold-leaf electrometer is much increased by an improvement in the insulation of the leaves, which we owe to the late Mr. Singer.

The instrument is constructed as usual, with a glass cylinder surmounted by a brass cap, but the 
insulation is made to depend upon a glass tube, about four inches long, and one-fourth of an inch internal diameter, covered both on the inside and outside with sealing-wax, and having a brass wire of a sixteenth or twelfth of an inch thick, and five inches long, passing through its axis, so as to be perfectly free from contact with any part of the tube, in the middle of which it is fixed by a plug

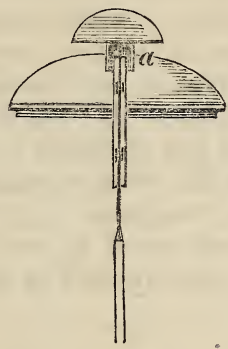

of silk, which keeps it concentric with the internal diameter of the tube. $a$ is a brass cap screwed upon the upper part of this wire; it serves to limit the atmosphere from free contact with the outside of the tube, and also defends its inside from dust; to the lower part of the wire the gold leaves are attached, and the whole mounted as usual, and as represented, in section, above. When such an electrometer is to be employed in atmospherical investigations, the contrivance described by Mr. Ronalds (Quar. Journ. ii. 249) may be conveniently applied to it.

The kind of electricity by which the gold leaves are diverged may be judged of, as before, by ap- 
proaching the cap of the instrument with a stick of excited sealing-wax: if it be negative, the divergence will increase; if positive, the leaves will collapse; upon the principle of the mutual annihilation of the opposite electricities; or that bodies similarly electrified repel each other, but that when dissimilarly electrified they become mutually attractive.

To ascertain the actual repulsive and attractive powers appertaining to weakly-electrified bodies, M. Coulomb has ingeniously availed himself of the principle of torsion, and has thus constructed his electrical balance. It consists of a fine metallic wire, $a$, one end of which is attached to the screw $b$, and to the other is suspended the horizontal needle $c$, composed of gum-lac or other non-conductor, and armed at one extremity with a gilt pith ball, counterpoised at the other end by

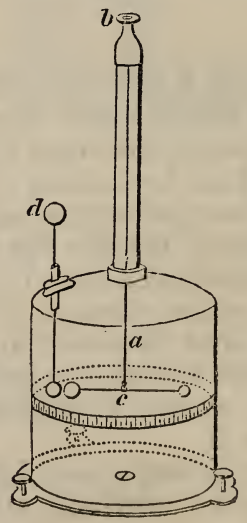


an index. The conductor $d$ is a small wire with a ball at each end, passing through the glass receiver in which the needle is suspended, and having its lower ball opposed to that of the needle. By the screw $b$, the two balls are brought into contact, and the index then points to $o$, on the divided scale of degrees. On communicating a very feeble electrical power to the conductor, it transfers it to the moveable pith ball, and repels it a certain number of degrees, proportional to the intensity of the acquired electricity, and measured by the power of torsion which it exerts upon the fine wire. By experiments made with this electrometer, it would appear that the electrical powers follow the law of gravitation, in being in the inverse ratio of the squares of the distances of the acting bodies. In the most delicate construction of the instrument, a single silk-worm's thread is used instead of the wire.

By means of the gold-leaf electrometer, constructed as above, we discover the existence of very slight states of electrical excitation, which elude all common methods of detection. If the cap of the instrument be gently struck with a silk handkerchief, a powerful divergence may be given to the leaves, resulting from the friction of the metal and silk; if blown upon by a pair of bellows, electrical excitation is also indicated, depending probably upon the friction of the air upon the metal; and it will be found in the sequel, that we conveniently avail ourselves of this instrument to detect the production of electricity resulting from a variety of other causes.

The nature of the electricity by which the 
leaves are repelled, may be discovered by approaching them either with an excited stick of sealing-wax, or a glass rod: if the divergence is increased by the former, it is negative; if diminished, positive; if increased by the glass, it is positive; if diminished, negative.

In consequence of the law of excitation by induction, which has been above explained, it is sometimes supposed that this method of merely approximating the instrument, instead of actually touching it by the excited wax or glass, might lead to erroneous conclusions; but if we examine a little more particularly into the state of things, we shall find that, consistently with the law of induction, electropolarity will be produced, and that consequently, the gold leaves will acquire the same electricity as that of the wax or glass, although the cap of the instrument may be in a different - state. Thus, let $\mathbf{N}$ represent a piece of excited

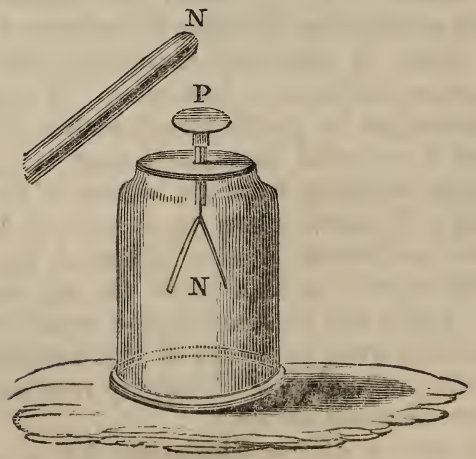


wax, negative; $\mathrm{P}$ the cap of the electrometer, positive; $\mathbf{N}$ the gold leaves, negative, that is, similarly electrified to the wax.

\section{§5.-Of Electrical Machines.}

In the preceding details we have availed ourselves of the simplest apparatus, in order to explain the leading and principal laws of electrical excitation; all the experiments are easily made, and their results include the most important facts connected with the subject, to which, therefore, in the sequel it will be necessary frequently to recur. For the further practical prosecution of these experiments, an electrical machine is indispensable; this, therefore, we may now proceed to describe.

The best electrical machine for experimental purposes, and for the general use of the young student, is represented in the following wood-cut. It is commonly called Nairne's machine, and may be obtained at most of the philosophical instrument makers.

It consists of a glass cylinder A, about 10 or 12 inches in diameter, and 15 to 20 inches in length, turning between two upright pillars of glass, B B, fixed to a stout mahogany base. Two smooth metal conductors, equal in length to the cylinder and about one-third of its diameter C C, are placed parallel to it upon two glass pillars DD, which are cemented into two sliding pieces of wood E, by which their distance from the cylinder may be adjusted. One of the conductors has a cushion, F, attached to it (by a bent metallic spring) 
nearly as long as the cylinder, and about one inch or an inch and a half wide, to the upper part of which is sewed a flap of oil-silk, G, which

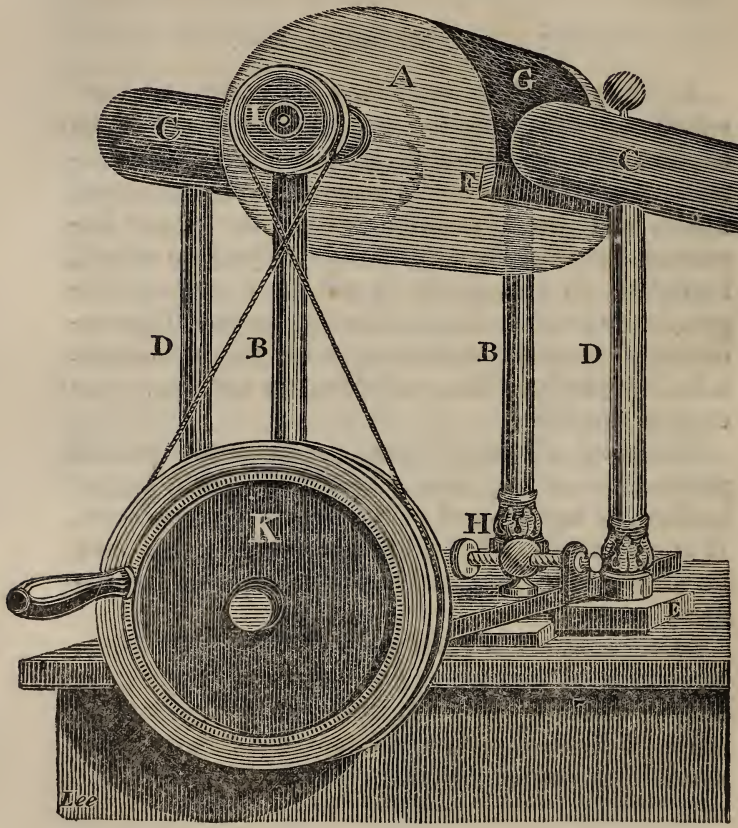

should reach from the cushion over the upper surface of the glass cylinder, to within about an inch of a row of points attached to the side of the opposite conductor. The conductor to which the 
cushion is attached is called the negative conductor; the other collects the electricity of the glass, and is called the positive conductor: $\mathrm{H}$ is an adjusting screw to regulate the pressure of the cushion upon the cylinder. The motion of the cylinder is in the direction of the silk flap, and may be most simply communicated by a handle attached at I, or, if necessary, by the multiplying wheel $\mathrm{K}$.

To put this electrical machine into good action, every part should be made perfectly clean and dry. The cushion is then anointed with amalgam, and applied by a gentle pressure to the cylinder. If positive electricity is required, it may be received from the conductor bearing the points, that supporting the cushion being uninsulated by a wire passing from it to the stand; if, on the contrary, negative electricity is required, it may be obtained from the insulated cushion cylinder, the other being uninsulated.

The best amalgam is composed of one part of tin and two of zinc melted together, and mixed, while fluid, with six parts of hot mercury in an iron mortar. This mixture is triturated till it becomes a fine powder, which is then formed into a tenacious paste with hog's lard.

Another form of the electrical machine, usually called the plate machine, consists of a circular glass plate A, mounted upon an axis, and rubbed by two pairs of cushions, as shewn at B B. The brass conductor $\mathrm{C}$ has its points opposed to the plate, and is insulated by the glass stem D: EE are double pieces of oil-silk passing from the cushions to near the points. The whole is sup- 
ported by a stout mahogany frame, and motion is given to the plate by the winch $\mathbf{F}$.

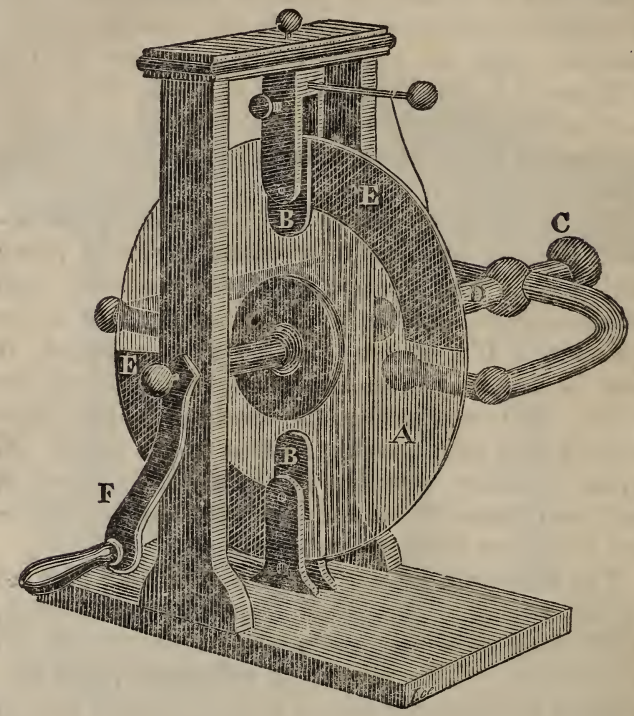

These electrical machines have considerable power; they are easily cleaned and excited, and are more portable than the cylinders; but as they cannot be conveniently insulated, the negative electrical power cannot be well exhibited, so that for the purposes of experimental research the former machines are preferable.

When either of these electrical machines have 
been for some time out of use, they require to be thoroughly cleaned: for this purpose the conductors and cushions must be removed, and the cylinder or plate cleansed with a little whiting and spirit of wine; it must then be wiped perfectly dry with a clean, warm, and soft cloth. It is a common custom to set the machines before the fire, but this is apt to soften their cemented joints, and often endangers the plate or cylinder; the former especially is often cracked by incautious warming. Dryness rather than warmth of the air is requisite for the activity of the electrical machine; it should, therefore, be kept in a dry room. When the glass parts of the machine are perfectly clean and dry, the cushions, freshly amalgamated, may be applied; their pressure upon the cylinder or plate should be very moderate, and they also should be dry and warm.

When the electrical machine is in good order, and the atmosphere dry, it produces a crackling noise when the plate or cylinder is turned, and flashes and sparls of light are seen upon various parts of the glass, passing from the cushion to the conductor: if the knuckle be held near the conductor, sparks pass to it through some inches of air, with a peculiar noise, and excite a slightly painful sensation in the part upon which they are received.

It is conjectured that the cause of the light thus perceived, is the sudden compression of the air or medium through which the electricity passes, and it is always probably attended by a proportionate elevation of temperature, as is shewn by the power of the spark to inflame spirit 
of wine, fulminating silver, and other easily inflammable compounds.

In these electrical machines the surface of the glass becomes positive, by friction upon the rubber or cushion, which becomes negative; and as the cylinder or plate is kept in progressive motion, it follows that, provided there be a means of carrying off its positive electricity, it will continually receive a new supply from the rubber; if, on the contrary, no means of conducting it away be resorted to, it will perpetually annihilate itself by retransmission to the cushion. But it follows, that when the cushion is insulated, the abduction of electricity must be very limited: hence, to the full and perfect operation of the electrical machine, two circumstances must co-operate; there must be a means of withdrawing the generated electricity from the surface of the glass, and of preserving the supply to the cushion: of these, the former is effected by the opposed points and prime conductor; the latter, by connecting the cushion by a metallic chain or wire with the earth. We accordingly observe, in examining the operation of the electrical machine,-1st. That the electricity in either conductor is extremely feeble when they are both well insulated. 2ndly. That when one conductor is uninsulated, the power of the other is proportionately augmented; in the positive conductor, because then the other draws uninterrupted supplies from the earth; and in the negative conductor, because the positive freely transmits, and hence is left in a state rapidly to receive. 3 dly. That the cushion and the surface of the glass are always in opposite states. 4thly. 
That the negative and positive electricities excited, bear precisely that relation which enables them, when combined, to neutralize each other: in other words, that the deficiency of electricity in the one conductor, is exactly such as to be restored by the redundancy in the other.

The law of electrical attraction and repulsion corresponds with that observed in magnetism, the similar poles of magnets repelling and their dissimilar poles attracting each other.

The appearance of the electric light is modified by the density of the medium through which it passes. In common air, short sparks are straight, or nearly so, and long ones zigzag; the former are brilliant, especially at their extremities; the latter usually of a paler or redder hue. In condensed air, the electric spark is bright and white; in rarefied air, it is of a reddish tinge, and faint and divided; in the more perfect vacuum of a good air-pump it is of a purplish hue, and only visible in a dark room. In a good Torricellian vacuum the light is faint blue; and in the most perfect vacua which can be obtained, it is scarcely visible, and of a greenish tint. In different gases, the electric spark appears most brilliant in those which are most dense ; in hydrogen gas, it is faint and red; in carbonic acid, it is vivid and white.

By the aid of Nairne's electrical machine, all the phenomena of excitation, and of attraction and repulsion, may be very conveniently studied. The conductor attached to the cushion is negative, and that opposed to the cylinder positive; the former, therefore, represents the sealing-wax, and the latter the glass, employed in the experiments 
above described. If we attach two pith balls to either conductor, they will of course repel each other; but if one be attached to the negative, and the other to the positive conductor, they will attract each other. If the two conductors be connected by a metallic wire or chain, all electrical appearances cease, shewing that the negative and positive powers are exactly such as to neutralize or annihilate each other.

Whenever a spark passes, it announces the annihilation of the opposite electrical states, they having been previously brought into existence by induction. Thus, if I hold my knuckle near the positive conductor of the electrical machine, it immediately becomes negative; and when these opposite states have acquired a certain degree of intensity, a spark passes from one to the other, and the state of excitation is at an end.

\$6.-Further Illustrations of Electrical Attraction, Repulsion, and Induction.

The statement of facts respecting electrical attraction, repulsion, and induced electricity, admit of many elegant and amusing illustrations by the electrical machine; the apparatus for which, with instructions for its use, will be found in most of the repositories of the philosophical instrument makers.

Among the most entertaining, are the head of hair, which stands on end when electrised, in consequence of the repulsion that takes place throughout the similarly electrified hairs. A similar experiment may be made by placing a person 


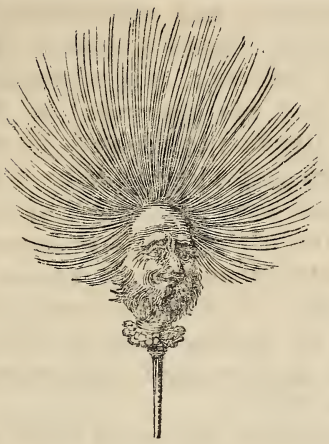

upon an insulated stool, in connexion with the conductor of the electrifying machine; his hair will diverge as in the above figure.

The following is a sketch of the simplest form of the electrical peal of bells, which ring

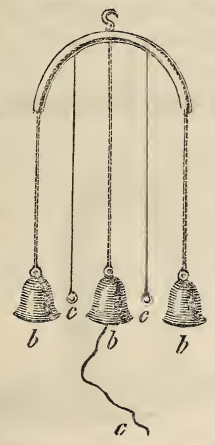


by the attractions and repulsions of small clappers suspended between them. The bells are arranged as follows: $a$ is a brass hook and wire by which they are suspended to the conductor; $b$ the bells attached to the wire by a brass chain; $c$. the clappers suspended by silk. The electricity received at $a$ is slowly dissipated by the edges of the bells, the ringing of which is occasioned by the alternate attractions and repulsions of the insulated clappers. A better arrangement consists in suspending the central bell by silk in the same way as the clappers, and connecting it with the table by the wire $c$.

Dancing figures, cut out of light paper, may be made to exhibit very ridiculous movements by a little management in turning the machine. For this purpose, suspend a brass plate from the conductor of the machine, and beneath it, at the distance of four or five inches, place a similar brass plate connected with the ground; on this put the paper figures; when the upper plate is electrified, they rise and perform a dance by their motion between the plates.

A good experiment illustrating attraction and

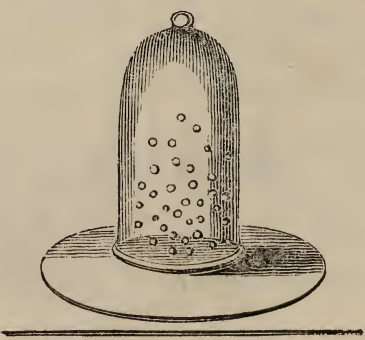


repulsion, consists in attaching a wire, with a blunt point, to the prime conductor, and holding over it a clean dry bell-glass, the interior of which will thus receive a superficial charge ; if it be then set upon the table over a dozen pith balls of different sizes, they will dance up and down in it for several minutes, until they have nearly carried off its redundant electricity.

Among the experiments particularly illustrative of electrical induction, we may notice the luminous tubes and words, in which a number of sparks are made to pass between small pieces of tin foil, so arranged as to produce in a darkened room a variety of figures and devices. In the luminous tube, a number of small spangles of tin foil are pasted spirally round a tube about half an inch in diameter, and within a very short distance of each other ; upon holding it to the prime conductor of the machine when in good action, a beautiful succession of small sparks is seen between each of the little discs of foil. Now, in all cases, before a spark can pass, the opposite electrical states must be brought into action, and they are here produced by induction throughout the series of spangles, the passage of the sparks indicating the momentary annihilation of the electricities thus excited. Let the annexed figures, for instance, represent three of the discs or spangles: it follows, then, that 1 being held

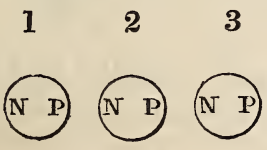


near to the positive conductor, will become polar, as represented by the letters $\mathrm{P}$ and $\mathrm{N}$; and the whole series, whatever may be their number, will be thrown into the same state as the conductors represented in the figure at page 172 , and remain so till the spark passes.

\$7.-Of the Dissipation "and Reception:of Electricity by Points.

The influence of points in receiving and carrying off electricity has already been adverted to, and may be further elucidated by the help of the electrical machine. If we hold a pointed wire to the prime conductor, it rapidly and silently draws off its electricity, and sparks cannot be taken from it by the knuckle, or a brass ball, while the point is in its neighbourhood; or if we attach a pointed wire to the conductor, there is the same disappearance of electrical accumulation ; and on holding the hand near the point, we perceive a peculiar coldness, or wind, as it were, which has been called the electrical aura: it is even suffi-

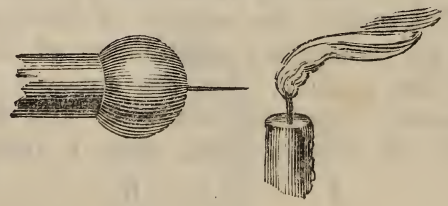

cient to blow out a candle held near it. This arises from the power possessed by the point, of indefinitely augmenting the intensity of the elec- 
tricity, which it communicates to the air, and which, consistently with the usual laws of repulsion, immediately passes off, or is repelled, and this rapid recession causes the current which is perceived.

If the point itself be moveable, it will of course be itself repelled, and upon this principle a variety of ingenious apparatus has been constructed, such as fly-wheels, orrerys, and so on. A simple instance is shewn in the annexed cut, in which a fly with four arms, each terminating in a point at

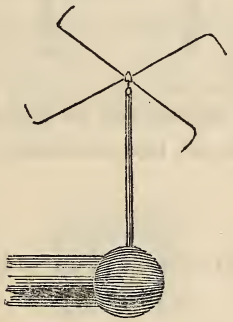

right angles to the arm, is balanced at its centre upon a pointed wire stuck into the prime conductor. The escape of the electricity from the points, and its action upon the adjacent air, causes the fly to revolve rapidly.

If these experiments be made in the dark, it is observed that a point held towards the negative conductor has the appearance of giving out a brush of light; but when held to the positive conductor, a luminous star or point is all that is observed. These appearances have been supposed to demonstrate the existence of an electric fluid, 
which, in the one instance, is supposed to be escaping from, and, in the other, received by, the

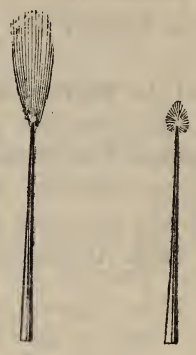

point: but these affections of light cannot be admitted as proofs of the existence of any distinct form of matter.

§8.-Of Induction through Glass-Construction of the Leyden Jar.

From what has been said respecting electricity by induction, and the electrical accumulation dependent upon it, it will appear that the intervention of conductors and non-conductors is requisite to the existence of the phenomena. In the cases cited, the non-conducting medium through which it takes place is the air; we shall now shew that induction may also take place through glass.

For this purpose it is only necessary to excite one side of a clean, dry pane of glass, by rubbing it with a piece of silk; and on holding a feather or pith-ball to the other side of the pane, it also 
will be found strongly electrical. If we now examine the nature of the electricities by which the glass plate is charged, it will be found that the rubbed side being positive, the opposite side is negative.

In the same way we may charge a plate of glass with the opposite electricities, by holding it to the prime conductor of the machine: in this case sparks will often appear to pass through the glass ; but, on removing it, the side which has touched the conductor will be found positive, and the opposite side negative.

As glass is a non-conductor, it is difficult to spread the electricity equally over its surface: this, however, may easily be effected by coating.

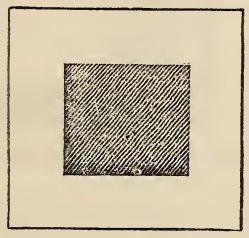

both sides of the pane with thin tin-foil to within about an inch and a-half or two inches of the edge, as shewn in the above cut. If we now communicate electricity to one side of the plate, the other, not being insulated, will become oppositely electrical, and in this way a charge will be acquired by the plate: if we now, by means of a bent wire, make a communication between the two sides of the coated pane, a bright spark will 
pass, and the accumulated electricities will be immediately annihilated.

We avail ourselves of arrangements of this sort for the purpose of accumulating electricity, which may thus be accomplished to a great and powerful extent; but the pane of glass is an inconvenient form of apparatus for the purpose, and we usually substitute for it a phial or jar, as represented below, coated inside and out with tin-foil to within three or four inches of its mouth. A brass wire surmounted by a knob is

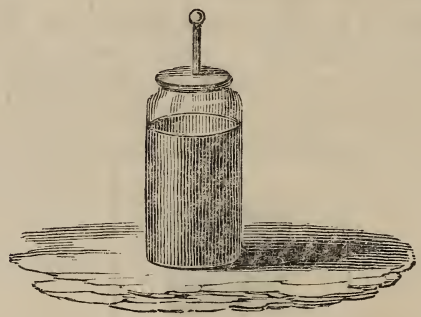

then made to communicate with the interior coating, and the apparatus is ready to receive its charge of electricity, precisely upon the same principle as the pane of glass. This is called the Leyden jar or phial, the method of arrangement having been accidentally discovered at that university. The glass should be thin, and, as far as possible, uniform throughout; for phials or jars which are very thick, or much thicker in one place than another, are unfit for the purpose.

If we now hold the jar by its outer coating, 
and present the knob near to the conductor of the machine, a succession of sparks will pass into it, at first very rapidly, afterwards more slowly, and at length ceasing altogether. The jar is then charged, and if a communication be now made by a metallic wire, from the outer to the inner coating, a bright spark, attended by a snapping noise, will pass from the knob, after which no further effect will be produced, the jar having been discharged.

The wire used for this purpose should be mounted with balls at its ends, and is usually called a discharger, $a$; it is sometimes jointed,
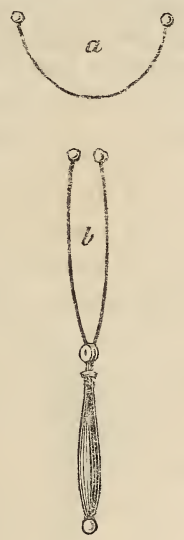

for the convenience of applying it to large and small jars, and mounted upon a glass handle, $b$. If, having charged the jar, and holding its ex- 
terior coating in one hand, we touch the knob with the other, the spark passes as before, and we perceive a peculiar and very painful sensation at the wrist and elbows, and across the breast, which is called the electric shock. It is painful for the moment only, and leaves little other permanent impression than that resulting from fear or surprise.

In charging the jar as above described, if we keep it in contact with the conductor, and the machine is in good action, we observe, after a time, that flashes of light pass across the uncoated interval, which are soon followed by a loud crack or explosion, and a long and brilliant spark passes from the wire to the coating. In this case the electricity has been accumulated to such a degree of intensity, as to discharge itself spontaneously over the uncoated interval.

To ascertain the relative charge which the jar has received, and its power of retaining that charge, we employ the quadrant electrometer, contrived by Henley. It consists of a round stem of metal, seven or eight inches long, surmounted by

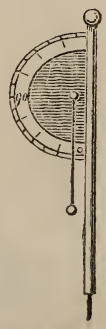


a ball, to which is attached a semicircle, most conveniently made of thin ivory: in its centre a pin is fixed, on which a slender slip of cane, about four inches long, with a pith-ball at its other end, turns freely, and traverses the semicircle as an index; the lower half of the semicircle is divided at the edge into ninety degrees. When this instrument is electrified, there is, of course, a tendency to repulsion between the moveable ball and the stem; the ball therefore recedes, and carries the index over the graduated circle, to a greater or less extent, in proportion to the intensity of the electric charge. The repulsion is at its maximum when the index stands at right angles to the stem, or at $90^{\circ}$ upon the scale. This electrometer may be fixed upon the prime conductor, or upon the knob of the jar. The latter is often a very convenient place for it, because, having charged the jar and removed it from the conductor, we see by the behaviour of the electrometer, how far the jar retains its charge.

In charging the Leyden jar many hundred sparks are observed to enter it, which, when it is discharged, are all concentrated into one; hence the brilliancy of its light, the loudness of the explosion, and the acuteness of the sensation which it produces.

When the jar is in direct communication with the conductor of the machine, it diminishes the intensity of its electricity, and consequently admits of a greater change in its elcctric state lefore any given resistance is overcome, or force of attraction manifested. This is shewn by attaching the quadrant electrometer to the con- 
ductor of the machine, which, when turned, immediately, causes it to rise to its full extent; but when the interior coating of the jar is in union with the conductor, the machine must be turned for some time before the rise of the electrometer attains its maximum.

The annihilation of positive by negative electricity, and the reverse, may be strikingly shewn by attaching the quadrant electrometer to a Leyden jar, and giving it a certain charge-say to twenty degrees-from the positive conductor; then transfer it to the negative conductor, and whilst apparently receiving a negative charge, the electrometer will gradually fall, and, at length, all electrical accumulation disappear: then, as the jar acquires its negative charge, it will again rise, and may be again discharged by transferring it to the positive conductor.

The power of the Leyden phial, as a source of electrical accumulation, being dependent upon the opposite states of its two surfaces, it is obvious. that one of them must always be connected with the ground, in order that it may acquire its charge. If we suspend a jar by its knob (a thin globular jar answers best for this experiment) to the positive conductor of the machine, its outer coating, being surrounded by dry air, cannot give. off any of its natural electricity, and consequently it will receive no charge so long as it remains. thus insulated; but if we hold the hand near the outer coating, sparks will pass off, and the jar acquire a charge. This is a good experiment, illustrating the Franklinian theory of electricity, which supposes the electric fluid to be present in 
all bodies, but to manifest itself only when its equilibrium is disturbed. Thus, when we charge a jar in the usual way, we add to the electricity of its interior coating, whilst its exterior throws off an equivalent quantity; so that the former becomes positive, or plus, and the latter negative, or minus: but if the jar be insulated, the electricity cannot escape from the outer coating, and consequently the jar cannot be charged.

The following experiment further illustrates this position:-Take two Leyden jars of similar size; insulate one of them by fixing it upon a glass stand $a$, and place the other on the table, with its

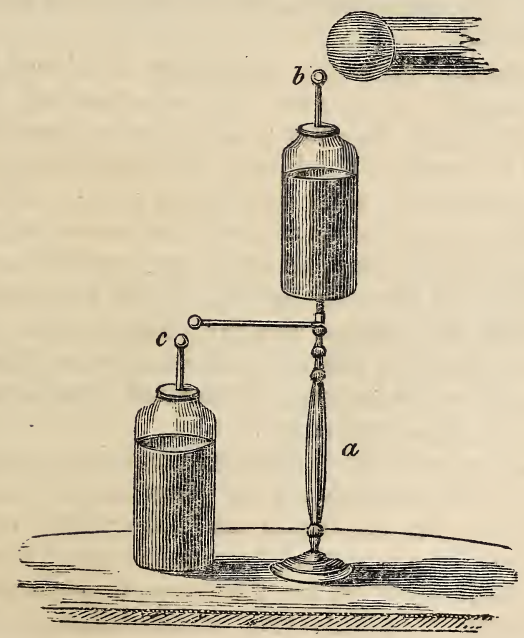


knob half an inch distant from the coating of the insulated jar, the knob of which should be placed at the same distance from the conductor of the machine. For every spark that passes to the knob of the first jar, $b$, there will be a similar spark pass from its coating to the knob of the second jar $c$, and both jars will become similarly and equally charged. As, therefore, the second jar is thus charged by sparks emitted from the exterior surface of the first, and as their charges are equal, it follows that for every portion of electricity added to one side of coated glass, a corresponding or equivalent portion leaves the opposite surface. The wood-cut just given shews the proper arrangement of the apparatus for this experiment.

Upon this principle a jar may be charged by the transfer of its own natural electricity from one surface to the other: for instance; suspend a jar to the positive conductor of Nairne's machine, and connect its outer coating by a wire with the negative conductor or rubber, the whole being in a state of insulation, so as to prevent the access of electricity from the earth. A few turns of the machine will charge the jar; that is, the electricity which is thrown into its inner surface by the positive conductor, is abstracted from, or supplied by, its outer surface.

This experiment is further instructive, as shewing the impermeability of glass to the electric fluid, for the conductor and the rubber of the machine are separated from conducting contact with each other by the thickness of the glass jar only, and a considerable accumulation of elec- 
tricity takes place, which the contact of the thinnest film of conducting matter, or the slightest fissure in the glass, would effectually prevent. When the glass of the Leyden jar is very thin, it does occasionally happen that it is perforated by a powerful charge : in this case a small round hole is observed in the glass, and the jar is rendered useless.

It has been said above, that a plate of glass not covered by tin foil, may be charged by contact with the prime conductor: the metallic coating, therefore, is not essential to its charge, but its principal use is, by its conducting power, to spread the electricity over the surface of the glass, and to enable it, when the discharge is made by uniting the opposite surfaces, to be carried off at once in a single spark. Jars are sometimes made with moveable coatings; these may be charged as usual, and the coating removed and again replaced, when it will be found that they still retain their electricity, which appears to adhere to the surface of the glass.

In speaking of the different states of electricity, the terms quantity and intensity are frequently used to indicate the different phenomena which it exhibits: the former implies the actual quantity of electric power in any body; and the latter, or the term tension, signifies the state of electricity indicated by the electrometer, and its power of flying off from surfaces, and passing through a certain stratum of air or other ill-conducting medium. The meaning of these terms will be more evident when we are considering the electricity of voltaic combinations : in the mean time, as re- 
gards the electricity of the machine, they may, perhaps, be further illustrated as follows. If we suppose a charged Leyden phial to furnish a spark, when discharged, of one inch in length, we should find that another uncharged Leyden phial, the inner and outer coating of which were communicated with those of the former, would, upon the same quantity of electricity being thrown in, reduce the length of the spark to half an inch; here, the quantity of electricity remaining the same, its intensity is diminished by one-half, by its distribution over the larger surface.

It is obvious, that the extension of surface alluded to in the last paragragh will be attended with a greater superficial exposure to the unelectrified air ; and hence it might be expected, that a similar diminution of intensity would result from the vicinity of the electrified surface to the ground, or to any other body of sufficient magnitude in its ordinary or unexcited state. That this is the case, may be shewn by diverging the leaves of the gold leaf electrometer, and in that state, approaching the instrument with an uninsulated metallic plate, which, when within half an inch of the electrometer plate, will cause the leaves to collapse; but on removing the uninsulated plate, they will again diverge, in consequence of the electricity regaining its former intensity. The same fact is shewn by the condensing electrometer. (See page 206.)

The power of the Leyden jar is proportioned to its surface, but a very large jar is inconvenient, and difficult to procure; the same end is attained by arranging several jars, so that by a communieation existing between all their interior coatings, 
their exteriors being also united, they may be charged and discharged as one jar. Such a combination is called an electrical battery, and is useful for exhibiting the effect of accumulated electricity.

The discharge of the battery is attended by a considerable report, and if it be passed through small animals it instantly kills them; if through fine metallic wires, they are ignited, melted, and burned; and gunpowder, cotton sprinkled with powdered resin, and a variety of other combustibles, may be inflamed by the same means.

\section{\$ 9.-Of the Electrophorus.}

There is only one other piece of common electrical apparatus which we shall here describe in detail, which is often used as a permanent source of electricity, and may sometimes be conveniently and economically substituted for the electrical machine, especially in the chemical laboratory; it is called an electrophorus. It consists of two

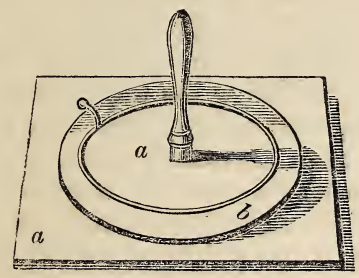

metallic plates, $a a$, with an intervening plate of resinous matter, $b$, for which equal parts of shell- 
lac, resin, and Venice turpentine, are generally used, the mixture being carefully melted in a pipkin, and poured, whilst liquid, into a wooden or metal hoop, of a proper size, placed upon a polished surface of glass or marble, from which it easily separates when cold; it should be about half an inch thick, and the smooth surface being uppermost, the lower side should be covered with tin foil, or attached to any other metallic plate ; a polished brass plate, with a glass handle $c$ attached to it, is then placed upon the upper surface of the resinous plate, and of rather smaller diameter. The resin is then to be excited with a piece of dry fur, and the instrument will be found to exhibit the following phenomena.

Upon raising the brass plate by its insulating handle, it will be found very feebly electrical ; replace it, touch it with the finger, and again lift it off by its handle, and it will give a spark of positive electricity. This process may very often be repeated without fresh excitation, which circumstance, as well as the nature of the electrical charge, shews that the electricity of the moveable brass plate is not directly derived from the resin, but that it depends upon induction: this is more obvious by considering the upper plate, not as in contact with, but merely very near the resinous disc, which, from the minute irregularities upon its surface, is really the case; the negative electricity, therefore, of the excited resinous plate, is communicated from a few points of contact to the brass plate, upon its first application, and then, the latter is precisely in the state of a conductor opposed to, but not touching, an electrified surface; 
and consequently in due condition to be rendered electrical by induction, when occasionally uninsulated by the contact of the finger.

With this instrument, one phenomenon of induction may be shewn, which cannot be so well exhibited by any other; namely, the increased capacity for electricity of the conductor under the influence of induction. The brass plate, when placed upon the resin, may be regarded as in a polar state; the lower surface near the resin being positive, the upper surface being negative. Upon touching the upper surface with the finger, it instantly acquires electricity, loses its polarity, and becomes positive, giving, upon removal, a positive spark to any conductor. That the quantity of electricity received from the finger, or other source, is equivalent to that given out, is shewn by the following experiment:- place the metallic upon the resinous plate, and touch the former with the knob of a Leyden phial; then touch the cap of an electrometer with the knob of the phial, and it will give a certain negative divergence to the leaves; raise the plate and present the knob of the jar to it, a spark will pass; and, upon applying the jar a second time to the electrometer, the leaves will entirely collapse, shewing the exact annihilation of the former negative, by the latter positive charge.

When the electrophorus is placed upon an insulating stand, its lower plate is always found in an opposite electrical state to the upper one, so that in this respect its plates resemble the coatings of a Leyden jar.

When an insulated surface is opposed to ano- 
ther which is not insulated, so as to be affected by it by induction, the electricity communicated to the former suffers a singular increase of tension or intensity on breaking the induction by removing the opposed uninsulated conductor; this property is strikingly exhibited in the following experiment:-Provide a brass plate, three or four inches in diameter, and drop upon its lower surface three small spots of sealing-wax; place it upon a similar plate, forming the cap of the goldleaf electrometer, from which it will be separated about a twentieth of an inch by the three small insulating legs of wax. Connect the upper plate with the ground, by touching it, and give a very feeble electrical charge to the electrometer, so as scarcely perceptibly to diverge its leaves; then suddenly remove the upper plate, by which the induction will be broken, and the tension of the electricity suddenly increased, so as to cause a very considerable divergence of the leaves.

The plates employed in this experiment have been called condensers. They are sometimes placed perpendicularly, and the uninsulated plate

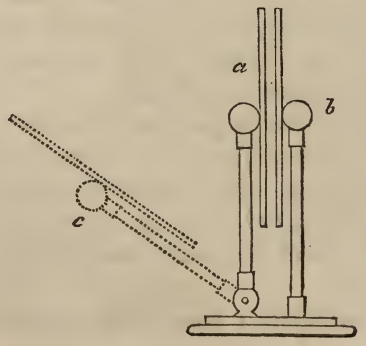


$a$ is supported by a wire and joint, so as to be brought as close as possible to the insulated plate $b$, without touching; the latter is in communication with the electrometer, and having received its charge, the moveable uninsulated plate is drawn back, as in $c$, and the intensity of the electricity displayed.

W Sometimes the condenser is directly attached to the electrometer, as shewn in the annexed cut: $a$ the insulated plate; $b$ the moveable plate in communication with the ground.

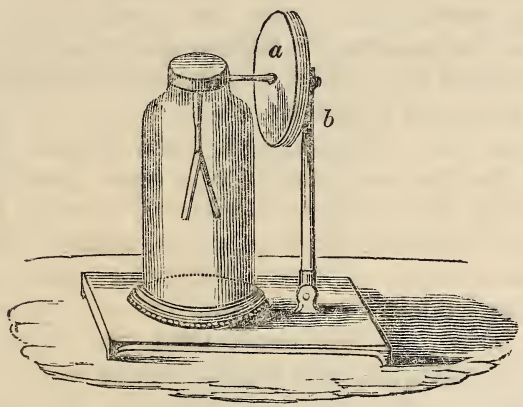

\$10.-Of Galvanism, or Voltaic Electricity.

We have now acquired such preliminary information upon the subject of electrical excitation, and of the phenomena exhibited by electrified bodies in general, as may enable us to understand some other, apparently more recondite and intricate cases of the production of this singular agent. In the mean time, we may observe, that scarcely 
any change in the general state of matter is unattended by a disturbance of the electrical equilibrium. Thus, the disruption of solid bodies, changes of temperature, changes of form, are all sources of electricity; breaking up a mass of sugar, tearing asunder the plates of mica, cleaving a piece of dry wood, heating certain minerals, evaporating liquids, and condensing vapours, may be cited as instances; and such of these as concern the chemist will again be noticed.

We shall now proceed more particularly to examine the excitation of electricity by the contact of metals: this gives rise to what has been termed Galvanism, or Voltaic Electricity, and is a branch of the subject bearing most importantly upon chemical research.

In the year 1790, Galvani, of Bologna, discovered that the transmission of a small quantity of electricity through the nerves of a recently killed animal, produced spasmodic convulsions of the muscles: he also shewed, that if the sciatic nerve of a frog be laid bare, and touched with a piece of zinc, whilst, at the same time, the muscle is touched with gold, that similar effects to those of electricity were produced whenever the metals were brought into contact, or connected together by conductors of electricity: if non-conductors were used to connect the metals, no spasm ensued. Cold-blooded animals retain this kind of excitability longer after death than others, and they are affected by states of electricity so feeble as not to be indicated by the most delicate electrometers. A feeble spark, or the most trivial charge of a Leyden phial, produce analogous 
effects. These facts soon attracted considerable attention, and were followed up with much diligence and curious results.

Frogs are the most convenient and susceptible animals for these experiments; but when they cannot easily be obtained others may be substituted*. Live flounders answer tolerably well, and

* The annexed sketch represents the legs of the frog prepared for galvanic experiments: the skin is removed, and the crural nerves $a a$ are then easily found, by gently sepa-

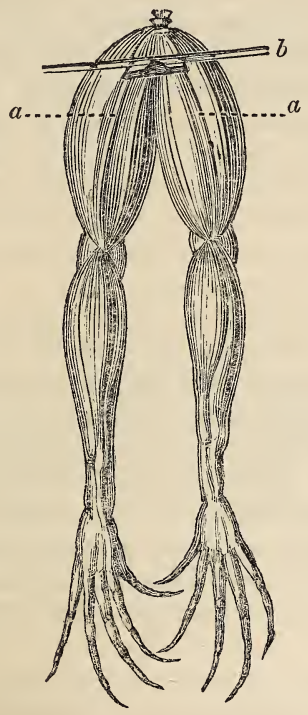


they may generally be procured at the fishmongers. Place the flounder in a plate upon a slip of zinc, and put a shilling upon its back; then, with a piece of wire connected with the zinc, make occasional contacts upon the shilling, and at each contact strong muscular contractions are produced.

In these experiments, if the separate pieces of metal be of the same kind, no effects are observed: to produce them, two different metals are requisite; and those metals which are most difficultly acted upon by acids, opposed to those which are, easily soluble, form, in general, the most powerful combination. Thus, gold and platinum produce scarcely any effect; but gold, or platinum, or silver, opposed to iron, tin, or zinc, are very effectual. This part of the subject will, however, be more explicitly inquired into afterwards.

There are many other experiments and illustrations relating to this subject, two or three of which may be here noticed.

If a piece of silver be placed upon the tongue, and a piece of zinc under it, no effect is perceived whilst the metals are kept apart; but, if their ends be brought into contact, we immediately perceive a saline taste, and a peculiar sensation resembling a slight electrical shock; sometimes, also, when the surface of the metals is extensive, a flash of light appears to pass before the eyes. This latter effect may be more certainly produced by placing one metal between the upper lip and

rating the muscles : $b$ is a silver wire passed under both the nerves, for the convenience of ensuring metallic contact. 
the gums, and the other upon the tongue, and bringing their ends together as before.

To account for these, and similar effects, Galvani supposed that the nerves and muscles of animals were in opposite electrical states, and that the spasms were produced in consequence of their annihilation by the metallic conductor. But this hypothesis was amply controverted, more especially by Volta, who shewed that the effect depended on the contact of dissimilar bodies, and not upon a communication between different parts of the animal. $\mathrm{He}$ shewed that contractions might be excited by the application of two different metals, but not by one only, and referred the effect to what has been termed the electromotive power of the different metals.

When two metals are brought into contact, it is found that, after removal, they are in opposite electrical states. Thus, when an insulated plate of zinc is brought into contact with one of silver or copper, the former is found, by the aid of a delicate electrometer, to be in a positive state, and the latter negative: hence it would appear, that a portion of electricity had passed from the least to the most oxidable metal. Of this transfer, or disturbance, the nerve and muscle of the frog is a very delicate indicator.

Place a large plate of metal upon the cap of the gold-leaf electrometer, and sift zinc filings upon it through a copper sieve, held by a glass handle: the leaves will diverge with positive electricity, and the sieve will acquire a negative state. Repeat the experiment, substituting a zinc sieve and copper filings for the copper sieve and zinc 
filings, and the divergence of the electrometer will be negative, the sieve being positive.

In these cases of electrical disturbance, the chemical properties of the metals are found to be materially affected, as may be proved by the following simple instances:-

1. Place a wire of silver and one of zinc in a wine-glass of dilute sulphuric acid, taking care that they do not touch each other; the zinc only will be acted upon by the acid, and the silver remains perfectly inert. Now, bring the upper ends of the wires into contact, and the silver will immediately become active; a stream of gas will be seen to issue from both of the wires.

2. Fill a wine-glass with a weak solution of sulphate of copper (blue vitriol), and place in it a small plate of clean iron, and one of silver: the iron will soon acquire a cupreous coating, but the silver remains bright until brought into contact of the iron, when it also immediately becomes plated with copper.

It will be necessary afterwards to dilate upon this important and curious effect of electricity; in the mean time a few analogous and familiar instances may be adduced: when the copper sheathing is affixed to the bottom of ships with iron nails, their heads soon corrode and give way, but when the nails are also of copper, no such destructive effect is observed: iron railings are apt to be decayed and dissolved when cemented into stone-work by lead; the effect is chiefly observed at the point of junction of the two metals: an iron pump put into a leaden cistern, or the junction of iron and leaden pipes, causes the corrosion of 
both the metals, but first and chiefly of the iron: when copper is immersed in sea-water it is soon corroded, and gives it a green tint; but if a piece of iron or zinc be in contact of the copper and the water, no such destruction ensues, the copper being protected by the iron.

What is commonly called ' a simple galvanic circle' is formed by partially immersing two different metals in a dilute acid, and making a communication between them, either by direct contact, or by some perfect conductor; one of the metals should be easily, and the other difficultly, or not at

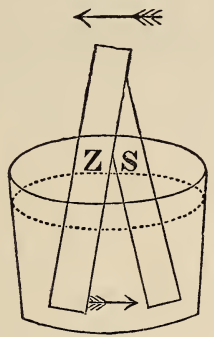

all acted upon by the liquid, and the more opposite the metals are in these respects, the more powerful are the electrical effects observed. In the above cut $\mathrm{Z}$ represents a plate of zinc, and $\mathrm{S}$ one of silver, partly immersed in very dilute sulphuric acid, and in contact at their upper ends; and by such arrangement a current of electricity appears to be put in motion, passing from the zinc to the acid, from the acid to the silver, and from the silver again to the zinc, as shewn by the direction of the darts. 
This arrangement may be modified, by immersing the plates separately in the acid, and connecting them by wires, as in the following woodcut: the circuit will thus be extended, but the electrical current will be found moving in the same direction, from the zinc towards the silver, and through the wires from the silver to the zinc, in

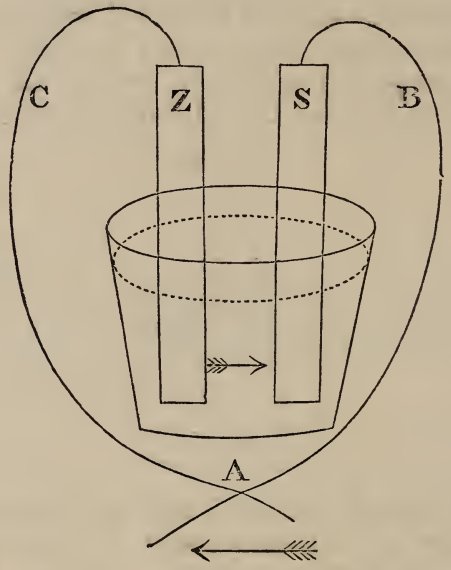

the direction represented by the arrows. In thus completing the circuit by wires, the electric current may conveniently be transferred through fluid and other bodies, and the circuit may be broken and restored at pleasure, by alternately separating and renewing the contact of the wires at A. It will be observed, that in consequence of the direction of the electric current in this arrangement, 
the wire $\mathrm{B}$, connected with the silver plate, is conveying electricity to the wire $C$, united to the zinc plate. Hence $\mathbf{B}$ is considered as positive, and $\mathbf{C}$ as negative.

Having now stated the simplest cases of electrical excitation by the contact of metals, we may proceed to examine the methods of accumulating it, and the effects which are thus produced.

If we merely multiply the series of copper and zinc plates, nothing more is effected than by a single pair, for it is evident that the negative copper being in contact with the positive zinc throughout the series, no accumulation can ensue.

But, if some imperfect conductor be interposed between each pair of plates, in that case induction is brought into action throughout the series, and a great increase of effect takes place, the phenomena of which are much dependent upon the nature of the interposed medium.

There are numerous electrical arrangements upon this prineiple; the simplest of them is what is usually termed from its inventor, De Luc's electric colimn. It consists of a number of alternations of two metals, with interposed paper; for this purpose thin paper covered with silver leaf may be used, punched out into circular discs of about an inch diameter, and alternating with similar discs of thin zinc foil, so arranged that the same order of succession-namely, zinc, silver, paper, zinc, silver, paper, \&cc., may be kept up throughout. About five hundred such alternations will be required to produce an active column, and they are most conveniently placed in a suitable glass tube, perfectly clean and dry within, and surmounted 
at each end with a brass cap, perforated by a screw, by which the plates may be pressed together, and which also serve as the poles of the arrangement; the screw at one end being in contact with the zinc plate, and that at the other with the silvered paper.

If we now hold this column by one of its brass caps, and with the other, touch the cap of the gold electrometer, a divergence will be communicated to the leaves, which will be found to be positive from the zinc end, and negative from the silver end of the arrangement ; that end of the column, therefore, to which the zinc surfaces incline, is called the positive extremity, or pole, and that to which the silver plates incline, the negative extremity.

If we place the column with each of its extremities in connection with the electrometer, as represented in the annexed cut, one of the instru-

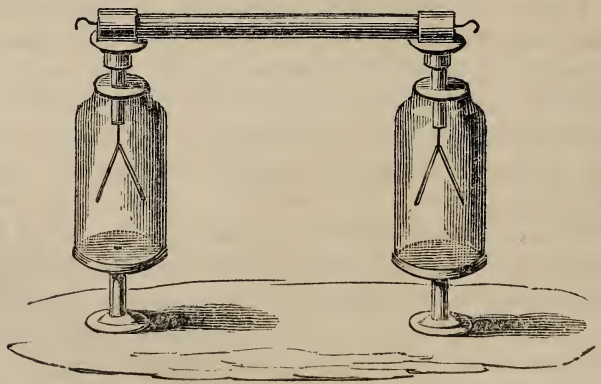

ments will be positively, and the other negatively diverged; and on making a direct communication 
between the two electrometers by a metallic wire, the divergence ceases, but again ensues when such communication is broken.

It will be found convenient in experiments with this column, to employ two, each containing 500 discs; these may be united end to end, so as to form one continuous arrangement. If we examine into the state of the electricity thus excited, we shall find it precisely that of a conductor under induction: that is, there is a positive and a negative pole, and a central point, which exhibits no electricity; upon making a communication between either end of the column and the ground, the electricity of the opposite end becomes proportionally exalted.

In the experiments we are now about to describe, it will be found convenient to employ an electrometer, in which each gold leaf is separately insulated, and so arranged as to admit of their being brought nearer to, or carried further from each other. Such an instrument is here represented

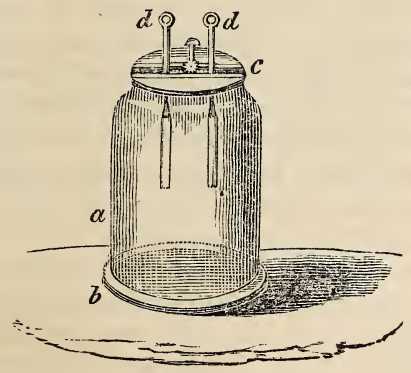


where $a$ is a glass cylinder mounted upon a brass or wooden base $b$; the cover $c$ is a thin piece of dry and varnished wood, with a slit in it, in which the glass tubes $d d$ slide backwards and forwards, and through which, brass wires pass, with a slip of gold leaf attached to each of their lower extremities.

If we now, having duly adjusted the distance between the gold leaves, bring the zinc ends of the columns in contact with each of the wires to which they are attached, the leaves will repel each other; the silver ends will produce a similar effect: but, if one of the gold leaves be connected with the zinc end, and the other with the silver end of the column, we shall observe that they immediately attract each other; and having thus, by contact, annihilated their opposite electrical states, they separate for a moment, and then again attract and separate as before, a kind of perpetual motion being kept up between the leaves, in consequence of the successive electrical charges communicated to them by the plates of the column.

Upon this principle a variety of amusing and curious instruments have been constructed, under the name of perpetual motions: thus, a small clapper may be kept constantly vibrating between two bells; or a light pendulum between two conducting surfaces; and these motions will continue as long as the column retains its electric activity, which is often for many months.

If one end of De Luc's column be connected with the interior, and the other with the exterior of a Leyden jar, the jar will receive a charge; its knob will powerfully affect an electrometer; and 
on discharging it by a wire, a small spark will be observed. To obtain this spark, or any luminous appearance, without the intervention of the jar, requires a very extensive series of the metallic alternations.

It will be observed, in regard to the curious instrument which we have just been using, that its electrical excitement is apparently independent of all chemical action, and therefore referable only to the contact of the metals; if the paper be damp, or the zinc or silver tamished, much of its activity disappears.

The original discoverer of a successful mode of accumulating the electricity generated by the motive power of the metals, was Volta, and to the instrument which he contrived for the purpose, the term Voltaic Pile or apparatus, is still applied, and the electricity which it generates is often spoken of as voltaic electricity. The pile consists of a number of plates of two different metals, alternating with moistened flannel or pasteboard, and arranged in regular order of succession. Zinc and copper are among the most powerful electromotors, and are the metals in general use: zinc and silver would furnish a more active series, but the use of the latter metal, in extensive combinations at least, is prohibited by its expense.

To construct the voltaic pile, take from thirty to fifty plates of copper, and as many of zinc, from two to four inches square, and place them in regular order, with an intervening piece of wet flannel of the same size, taking care that the regular succession of copper, zinc, flannel, copper, zinc, flannel, is kept up through the series. Having made 
this arrangement, connect a wire by perfect metallic contact with the lowest, or copper, and a similar wire with the uppermost, or zinc plate, having previously slipped a small piece of glass tube upon the wires, to serve as an insulating handle ; then, on bringing the upper wire in contact with the electrometer, it will diverge the leaves positively, and the lower wire negatively, and the same general phenomena will be observed as in De Luc's column, the imperfect conductor being, in the present instance, water instead of paper. If the fingers be thoroughly moistened, by rubbing them with salt and water, so as in some measure to overcome the non-conducting power of the cuticle, a slight shock will be perceived on touching the extreme wires with each hand: a spark is very rarely observed, even in a very dark room, and no decomposition of water is effected by immersing the poles near each other in that fluid.

If we now substitute a strong solution of salt in water slightly soured by muriatic acid, instead of pure water, for moistening the intermediate flannel, we shall not observe any change in the mere electrical power of the pile as indicated by the affections of the electrometer: the shock will be little more powerful; but a spark will be perceived, on bringing the poles together, more especially brilliant when taken between two points of well-burned charcoal attached to the wires from the top and bottom of the pile. Also, if we now bring the poles (which for this purpose should be of platinum wire) near each other, in a glass of water, gas will be abundantly extricated by each 
wire. Here, then, we observe, that the power of attraction and repulsion communicable by the pile, or, in other words, its intensity, is not increased by the chemical action going on between the acid liquid and the plates ; but, on the other hand, the quantity of electricity, as manifested by the generation of light and heat, is greater than before. This effect will presently be more particularly adverted to.

In the construction of the voltaic pile as just described, much inconvenience results from the method of arrangement, when the plates are very numerous ; and the weight of the upper part of the pile presses the moisture out of the lower part, and renders it comparatively inactive. To obviate this, each pair of plates is sometimes soldered together, and cemented in regular order, into a well-seasoned mahogany trough, and the intervening cells, which are about a fourth of an inch wide, are filled with water or other proper liquid, by, which the arrangement is rendered active.

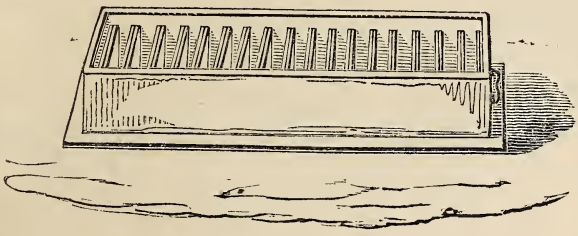

The apparatus thus constructed is easily filled and emptied, and may be kept clean by rinsing it out with water, after use. A wire, in contact with the 
last zinc plate, affords a positive pole; and, with the last copper plate, a negative pole.

Another form of this apparatus, also contrived by Volta, and called by him the 'Couronne des tasses,' consists in arranging a row of small glasses, containing a very dilute sulphuric acid (or other proper liquid), in each of which is placed a plate or wire of silver or copper, and one of zinc, not touching each other, but so connected, that the zinc of the first glass may be in metallic contact with the copper of the second, and the zinc of the second glass with the copper of the third, and so on throughout the series, as shewn in the annexed. cuts.
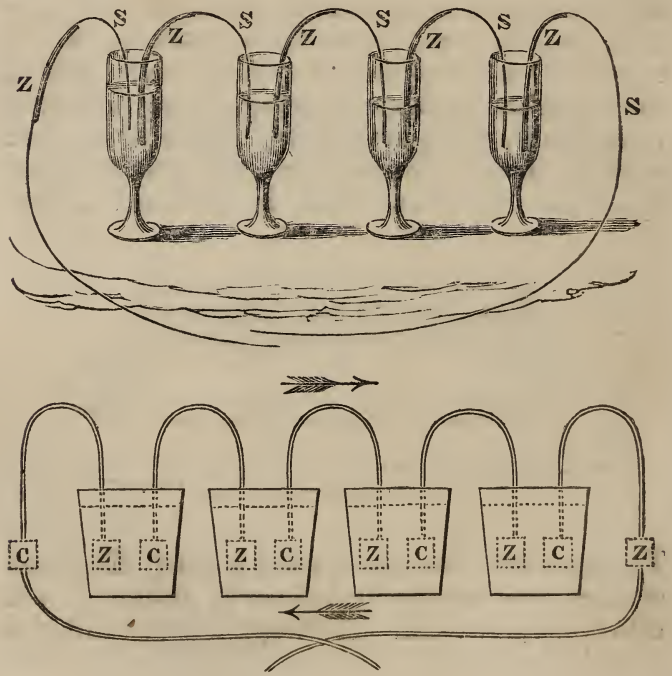
By arrangements of this kind, we can see what happens in each cell of the apparatus; and it will be observed, that when the extreme wires or poles are in contact, and a current of electricity therefore passing through the arrangement in the direction of the arrows, that each wire or metallic surface immersed in the liquid is actively decomposing water; whereas, when the connexion between the poles is broken, the copper and the silver cease to evolve gas, and it is much more sparingly elicited by the zinc. In other respects, the general properties of the apparatus thus arranged, resemble those of the other forms above described.

The voltaic apparatus now in most common use in the laboratory, is constructed upon similar principles : it consists of a trough of earthenware, with partitions of the same material. The plates, of copper and zinc, are soldered together at one point only, and each united pair of plates is so arranged as to inclose a partition between them; consequently, there is in each cell, a copper plate connected with the zinc of an adjoining cell, and so on, in regular succession. Each of these troughs usually contain ten pairs of four-inch plates, and the connecting piece of the last copper plate is elongated, so as to enable it to dip into the first cell of the next trough, when they are placed in rows endways to each other; it is there opposed to the ensuing zinc plate of another set. The plates are attached to a slip of baked wood, so that the whole ten may at once be lifted into or out of the cells. This construction has many advantages over the trough above described: it 
admits the fluid to remain in the trough, while the action of the plates may be suspended at pleasure, by lifting them from the cells; when the plates are corroded or injured, they are also easily replaced.

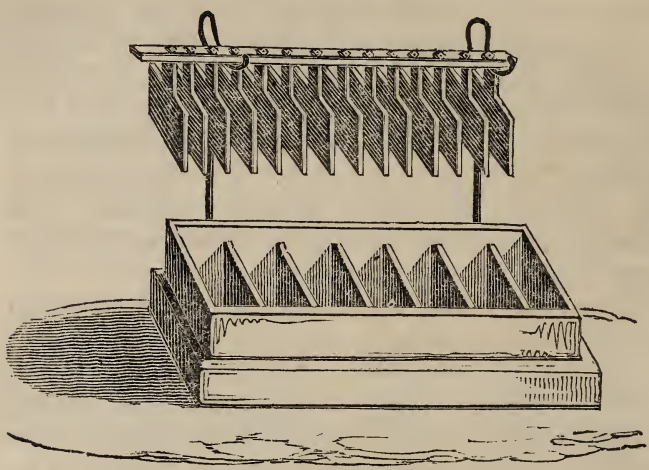

In consequence of both of their surfaces being exposed to the acid, they wear faster than the soldered plates ; but then they yield a more powerfully electric arrangement. Where many of these troughs are employed, so as to constitute a battery of large dimensions, it is necessary to be extremely attentive to the regular order of succession throughout the series: if any single trough be reversed, a great diminution of effect is the consequence. Much care must also be taken respecting the communications between the troughs, to prevent the accidental displacement of any of the junction plates. It is well to be provided with a few spare pairs of plates attached to each other by 
a sufficiently long copper strap, for the purpose of uniting the troughs, where their arrangement requires that they should be placed at angles to each other.

A considerable improvement in the construction of this apparatus was suggested in the year 1815 by Dr. Wollaston, and is now very commonly adopted ; at least where no very extensive series is required, and where it is an object to unite quantity with intensity. It consists in extending the copper plate, so as to oppose it to both surfaces of the zinc, as shewn in the annexed sketch,

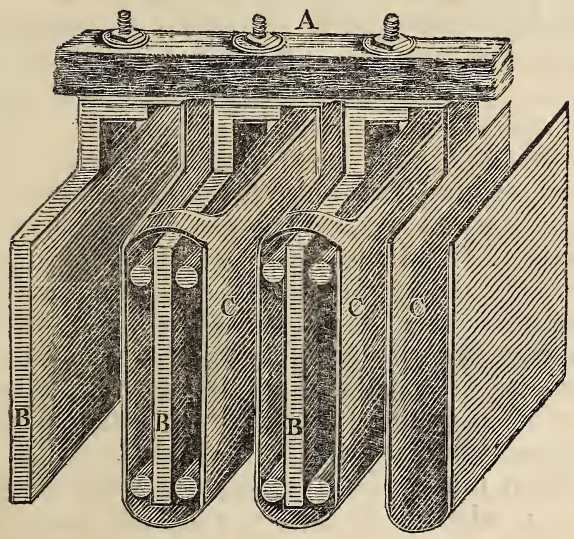

where A represents the bar of wood to which the plates are screwed; $\mathrm{BB}$ the zinc plates connected as usual with the copper plates C C, which are doubled over the zinc plates, and op. 
posed to them upon all sides, contact of the surfaces being prevented by pieces of wood or cork placed between them, which keep the plates about one-fourth of an inch asunder. Twelve troughs upon this construction will be found sufficient for all the ordinary experiments and illustrations in which the voltaic apparatus is used, and the plan supersedes the necessity of being provided with batteries of large plates.

It has already been remarked, that what may be called the common electrical effects of the voltaic apparatus, that is, its power of diverging the electrometer, and charging a Leyden jar, are most prominent when the troughs are well insulated, the plates clean, and the cells filled with common river water. Two thousand four-inch plates thus charged and insulated, gave sparks and shocks, and charged Leyden jars, and of course acted with corresponding energy upon electrometers : it was also curious to remark, that some time was required, after the electricities had been annihilated by the junction of the poles, for its restoration or reproduction; the sparks which it afforded were like those of a weak electric machine; the power of ignition upon charcoal points was feeble; and scarcely any effects were observed on immersing the poles in water ; nor was the electricity capable of igniting wire.

When this magnificent apparatus was charged with a mixture of ninety parts of water, and ten of a mixture of muriatic and sulphuric acids, it exhibited very different and extremely splendid effects. The shock was more permanent and intense to the feelings, but the power of 
giving divergence to the electrometer, and of charging jars, was not increased. Wires of metal were intensely heated, and if thin enough, and in short lengths, burned and fused. A piece of well-burned charcoal, cut to a point, was attached to each pole, and, on bringing these into contact, a most vivid light and intense heat were produced; and, on slowly withdrawing the points from each other, a constant current of electricity passed between them, through more than an inch of heated air, producing an arc of light of such intensity as scarcely to be borne by the unprotected eye, and of the form here represented.

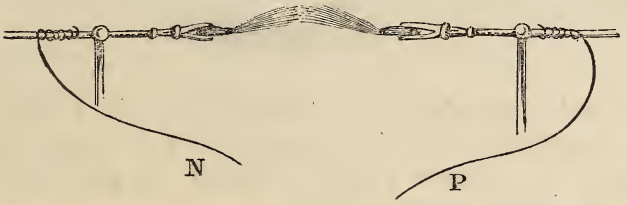

When the charcoal points were gradually withdrawn from each other, in a vessel exhausted of the greater portion of its air, the effect was yet more brilliant; the light acquired a beautiful purple colour, and the distance through which it passed was much extended, as shewn in the woodcut overleaf.

The extreme intensity of the heat, in this arc of flame, was rendered evident by exposing in it very difficultly fusible substances, which readily melted: even substances infusible by all ordinary methods were thus liquefied.

What may be termed the chemical energies of this apparatus were equally remarkable: when 


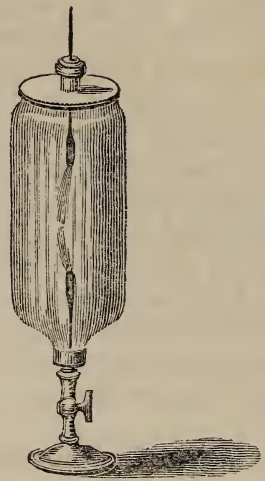

the poles were brought near each other in a vessel of water, that fluid was energetically decomposed, and other effects produced which we shall presently notice more in detail.

It is evident from the statement just made, that a most important influence is exerted over the energies of the voltaic pile, by the liquid between the plates. Pure water produces a certain series of electrical effects; these are considerably modified by dissolving common salt in it, or employing other saline liquids; but the dilute acids appear the best calculated to increase the quantity of electricity, by acting chemically upon one of the plates. If, for instance, we charge three single troughs, each of ten pairs of plates, with water, brine, and very dilute muriatic acid, the first will shew scarcely any signs of electricity; the second will give a feeble spark and shock; the 
third will ignite the charcoal points, producing a continuous star of brilliant light, and give a more powerful shock. The first will not decompose water; the second does it feebly; the third rapidly. It is obvious, therefore, that with a small number of plates kighly charged (that is, with dilute acid), we may obtain effects equivalent to those of a larger series feebly charged; the experimentalist, therefore, must regulate the strength of the charge according to the number of plates, and the effects which are required.

All the effects of electrical intensity, such as affecting electrometers, giving shocks, charging jars, decomposing water and saline solutions, and so forth, are very manifestly increased by increasing the number of the plates, but (the charge being in both cases the same) the production of quantity of electricity appears to be dependent upon the size of the plates. Thus, if a battery composed of thirty pairs of plates two inches square be compared with another battery of thirty pairs of twelve inches square, scarcely any difference will be perceived in their effects upon bad and imperfect conductors; their powers of decomposing water, and of giving shocks, will be nearly similar; but upon good conductors, the effects of the large plates will be very distinct from those of the small ones: the spark, and arc of light between charcoal points, will be much more intense and extensive ; and when the charge is transmitted through a fine platinum wire, much of it will be heated red hot, an effect which the small plates are quite inadequate to produce.

The following experiments are adduced by Sir 
H. Davy, as well illustrating these relative effects of quantity and intensity in the voltaic apparatus:

'Immerse the platinum wires connected with the extremities of a charged battery composed of twelve inch plates into water, and it will be found that the evolution of gas is nearly the same as that occasioned by a similar number of two-inch plates. Apply the moistened fingers to the wires, and the shock will be the same as if there were no connexion by the water. While the circuit exists through the human body and through the water, let a wire, attached to a thin slip of charcoal, be made to connect the poles of the battery, and the charcoal will become vividly ignited. The water and the animal substance discharge the electricity of a surface probably not superior to their own surface of contact with the metals; the wires and charcoal discharge all the residuary electricity of the plates; and if a similar experiment be made upon plates of an inch square, there will scarcely be any sensation when the hands are made to connect the ends of the battery, a circuit being previously made through water; and no spark when charcoal is made the medium of connection, imperfect conductors having been previously applied.'

For all common purposes of research and experiment, voltaic arrangements are usually most convenient, in which intensity is obtained, together with a due quantity of electricity; and upon this principle Dr. Wollaston's arrangement of the plates has been recommended as most economical and effective.

The most formidable battery of large plates 
that has hitherto been constructed, is that described by Mr. J. G. Children, in the 'Philosophical 'Transactions' for the year 1815. The plates were two feet eight inches wide, and six feet high, the copper being opposed to both surfaces of the zinc. These enormous plates were properly fastened to a beam of wood, suspended by counterpoises from the ceiling of the laboratory, so as to be readily and safely immersed into, or removed from the cells of acid, which were twenty-one in number, and their united capacities amounted to 945 gallons. A leaden pipe, threefourths of an inch diameter, was attached to the extreme plate at either end, and immersed into separate basins of mercury, by means of which perfect metallic contact was ensured. The charge consisted of a mixture of nitric and sulphuric acids, with thirty, or occasionally only twenty, parts of water.

When the poles of this arrangement were united, as shewn in the next page, by a platinum wire eleven-hundredths of an inch diameter, it became red-hot for a length of five feet six inches. In the same way, it ignited eight feet six inches of the same wire of forty-four hundredths of an inch; and a bar of platinum, one-sixth of an inch square, and two and a quarter long, was not only heated to bright redness, but fused at the end.

A number of intractable and very difficultly fusible substances, submitted to the high temperature occasioned by the discharge of this battery through charcoal points, exhibited indications of the most intense heat; but perhaps the most interesting results were obtained by transmitting the 


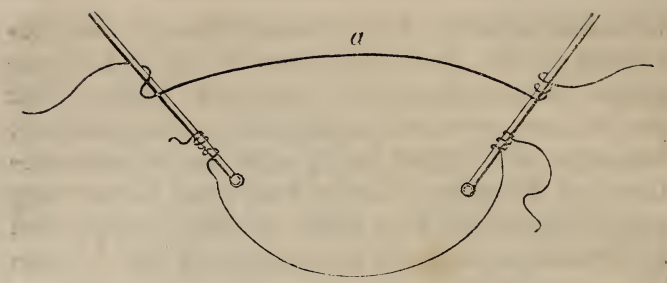

electricity through different metallic wires. For this purpose either single wires, or two wires of dissimilar metals were used, of equal diameter and length: one end of each was in contact with one. of the basins of mercury communicating with the poles of the battery, and the other end bent to an angle, and the wires connected continuously by hooking them together. The length of each wire was eight inches, and the diameter one-thirtieth of an inch.

Platinum and gold being thus connected, and introduced into the electrical circuit, the platinum was instantly made red-hot, whilst the gold remained unafiected.

With a similar arrangement of gold and silver wires, the gold was ignited ; the silver not.

With gold and copper, both metals were equally heated to redness.

With gold and iron the iron was ignited; the gold not affected.

Alternations of platinum and silver three times repeated, all the platinum wires were ignited, but none of the silver.

These experiments may easily be repeated with the smaller battery, the dimensions of which we 
have described above, and are extremely beautiful and instructive, especially the last: for this purpose select some fine silver and platinum wire, and cut it into lengths of about two inches; then form a continuous wire by soldering these lengths endways, in alternate order, and suspend it in a festoon, between two thick copper wires, forming the poles of the battery, having previously temporarily united

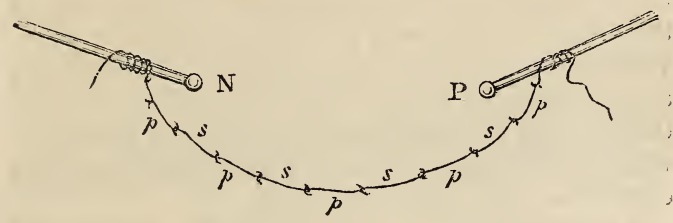

these poles by a thick copper wire, as is shewn. at $a$ in the preceding figure : on removing the latter, the electricity traverses the compound wire, and occasions the ignition of the platinum portions of it only, the silver being unaffected. 'The object of uniting the poles by a thick copper wire, whilst the compound wire is being attached, is to prevent the sudden fusion of the latter at the point of contact, which often happens when this precaution is not taken.

What, it may now be asked, is the cause of this extraordinary appearance? why is one wire more intensely heated than the other, when they are both of the same diameter, and apparently transmitting the same quantity of electricity? It has been plausibly suggested by Mr. Children, that the effect is the result of the different conducting powers of the metals; that platinum conducts 
electricity less perfectly than silver, and that, consequently, the electric fluid, meeting with greater resistance in its passage through that metal, elicits light and heat; while, on the other hand, passing with comparative facility through the silver, no such effect is there observed. It is obvious that this explanation rests upon the supposition, that resistance to the passage of electricity occasions the development of heat; and as this must be inversely as the conducting power, when any two of the wires, connected continuously, are placed in the circuit, that which is the worst conductor must be most heated; and silver, therefore, which is the best conductor, is not heated red when connected with any other metal.

In proof that resistance causes the evolution of heat, the chemist we have just quoted observes, that whenever a perfect communication is established between the poles of the battery, the electricity circulates without producing any visible effect; but if it meet with resistance in its passage, it manifests itself by chemical action, or by the evolution of heat, or both. Thus, if a bar of metal be connected with one pole of the battery, and its extremity immersed in a basin of mercury connected with the other pole, at the instant the surfaces come in contact, heat and light are evolved, which cease as soon as the bar, if it be of sufficient size, is plunged beneath the surface of the quicksilver. If the circuit be completed by two pieces of charcoal, the evolution of heat and light is permanent as long as their surfaces remain in contact, because that contact can never be so perfect as to oppose no resistance to the electricity: whereas, in the case of the bar. 
of metal and the mercury, it soon becomes complete, and the current is then uninterrupted.

We have now, then, enumerated some of the leading phenomena presented by the voltaic pile, or battery, limiting ourselves chiefly to the consideration of its electrical energies; and it is obvious that, in its form or construction, it must vary according to the particular purposes to which it is to be applied. There are numerous cases in which quantity of electricity is the principal requisite with the experimentalist, and in such, large plates and few in number will be employed; a single pair, or two pairs, are often sufficient for various researches of this kind, especially when constructed upon Dr. Wollaston's plan. On the other hand, where intensity is required, we are obliged to resort to numerous alternations of plates comparatively small, and these arrangements are principally employed where our object is to act upon bad conductors, and especially to avail ourselves of the chemical powers of electricity; to this subject we may now more particularly turn our attention.

\section{$\oint$ 11.-Of the Chemical Effects of Electricity.}

The influence of electricity as a decomposing agent was noticed in some of the earliest experiments that were instituted with the voltaic pile; among them, its action upon water excited particular attention. If two wires of platinum*, connected with the poles of the battery, be brought near

* This metal is selected in preference to others, as not being acted upon by the elements evolved from water. 
each other in a glass of water, a regular stream of gas will issue from each of them, which in an apparatus similar to the following, may be col-

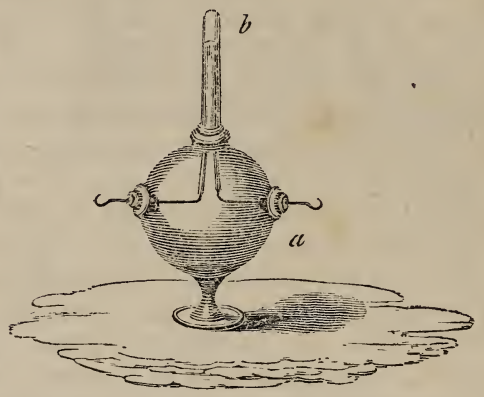

lected, and submitted to examination. $a$ is a glass globe with three apertures; two of these (on the sides) are fitted with corks perforated by glass tubes of such length as to approach the centre of the globe, and traversed by platinum wires, which are flattened out at the ends, and turned upwards, within a tenth of an inch of each other. The tube $b$ is inverted into the neck of the globe, which it fits loosely, so as to admit of the oozing of a portion of water. This apparatus is then filled with water, and the protruding ends of the platinum wires made to communicate with the negative and positive poles of the voltaic apparatus; gas immediately issues from the metallic surfaces in contact with the water, and bubbles up into the tube; the displaced water trickles out at the neck of the globe. It will be observed, that 
twice the quantity of gas escapes from the negative, as compared with that from the positive pole; and the tube will be found to contain a mixture of hydrogen and oxygen gases, in the proportion of two volumes of the former generated at the negative pole, and one of the latter at the positive pole. If a lighted candle be brought to the mouth of the tube, the mixed gases explode and disappear, reproducing water, as we shall more fully shew in the sequel.

By a simple modification of this arrangement, the evolved gases may be collected in separate tubes; as, for instance, if we employ a globe with two orifices, each having a tube so adapted to it

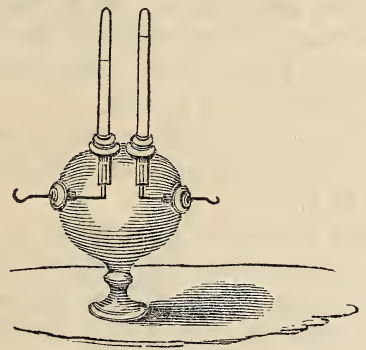

as separately to receive the gas from each wire. It will then be found that two volumes of hydrogen gas, and one volume of oxygen gas, are respectively collected in the tubes inverted over the negative and the positive wires. Upon the approach of a taper, the hydrogen gas will burn quietly with a blue lambent flame; and the oxygen 
may be recognised by immersing into it the glowing wick of a small piece of green taper, which will instantly kindle into flame.

These experiments are here merely adduced in illustration of the chemical or decomposing powers of electricity; and they shew us, that under whatever circumstances water is decomposed, the hydrogen is evolved at, or attracted by, the negative, and the oxygen by the positive pole. They are, however, further important, as establishing the composition of water; a liquid so universal in its agencies, and so important in its effects, that the chemical student cannot too soon become well acquainted with its nature. If we reduce the volumes of the gases to their respective weights, we shall find that, if we call the volume of hydrogen $=1$, the half volume of oxygen will be $=8$, and, consequently, nine parts of water will consist of one part by weight of hydrogen, and eight parts by weight of oxygen, for the specific gravity of oxygen to hydrogen is as 16 to 1 .

There is another conclusion to be drawn from this electro-chemical decomposition of water, which it may be right here to bear in mind, but which must be more amply developed afterwards. It has been above demonstrated, that bodies mutually attractive are oppositely electrical, and that when mutually repulsive they are similarly electrical: now, as the hydrogen is here attracted by the negative pole, we infer that its own inherent electrical state must be positive; and, on the other hand, the oxygen being attracted by the positive pole, it is assumed to possess the opposite or 
negative state. Hydrogen, therefore, and all substances which, like it, are repelled by positive, and attracted by negative surfaces, are classed together under the term electropositive bodies; and oxygen and other substances which are repelled by negative, and attracted by positive surfaces, are called electronegative bodies.

If, instead of using pure distilled water in the experiments just described, we employ hard or pumpwater, we shall find, that the saline ingredients, always present in such water, are also decomposed, and that their elements are separated according to certain laws.

This fact may be more strikingly illustrated by dissolving some salt, of known composition, in water, and electrising the solution, as in the following experiment. Provide a piece of glass tube, bent at an angle, and placed in a wine-

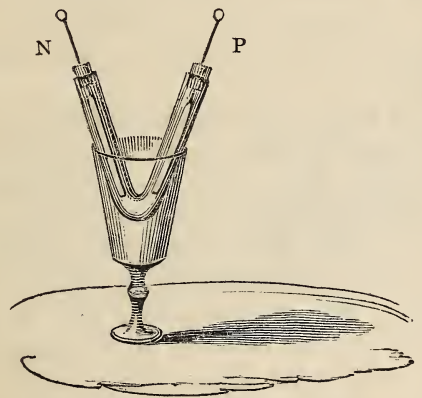

glass, to serve for its foot or support. Fill this syphon with the blue infusion obtained by mace- 
rating the leaves of the red cabbage in boiling water, and put into it a few crystals of sulphate of soda; then place a long narrow strip of platinum foil in each leg of the syphon, taking care that they do not come into contact at the elbow of the tube, and connect one of these with the negative, and the other with the positive pole of the pile: in a few minutes the blue colour will be changed to green in the negative side, and to red in the positive side of the tube, indicating the decomposition of the salt, the alkali or soda of which is collected in the negative, and the sulphuric acid on the positive side. Reverse the poles, and the colours will also gradually be reversed. In this and analogous experiments, it is found that, whenever a neutral salt is decomposed by electricity, the oxide or base is attracted by the negative pole, and the acid by the positive pole. The bases, therefore, in their electrical relations, rank with hydrogen, and the acids with oxygen: the former are electropositive, the latter electronegative.

The mostinsoluble and difficultly decomposable salts may be made to render up their elements in the same way. Let us, for instance, substitute for the sulphate of soda in the preceding experiment, a little finely-powdered sulphate of baryta, which is quite insoluble in water: this, when in contact with the platinum, will be decomposed; baryta will be evolved at the negative pole, and there render the liquor green; and sulphuric acid at the positive pole, rendering it red.

The two last experiments may be instructively varied as follows :-fill two wine-glasses, con- 
nected together by a syphon of large bore, with the blue infusion, containing a little sulphate of soda dissolved in it, and invert in each glass a

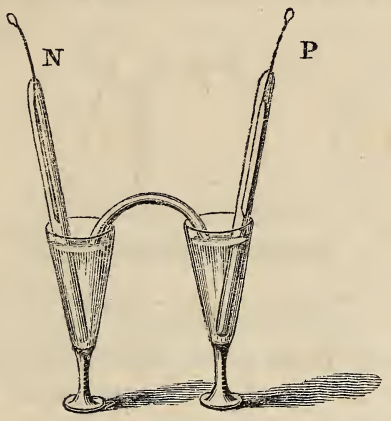

tube of the same solution, into the upper ends of which platinum wires are inserted, as shewn in the above cut: connect these wires with the negative and positive poles, and it will presently be remarked that, notwithstanding they are in separate vessels, the blue liquor in them will, as before, be rendered green and red; and if the experiment be continued for a suficient length of time, the whole of the alkali of the salt will have passed from the side $\mathrm{P}$ to $\mathrm{N}$, and the acid from $\mathrm{N}$ to $P$. The acid and alkali must, in this case, obviously traverse the connecting syphon in opposite directions, without uniting; so that, under the infuence of electrical attraction, the usual chemical affinities are suspended.

The decomposition even of the sulphate of baryta may be effected in the same way: for this 
purpose, provide two insulated discs, or very shallow cups of platinum, one of which is to be put into communication with the negative, and the other with the positive end of the pile; place

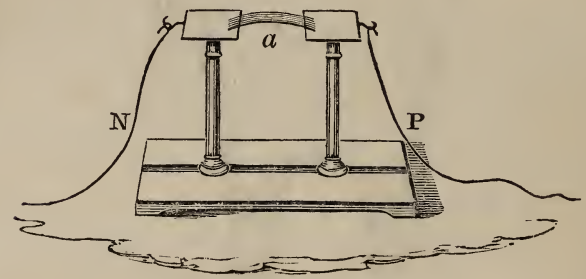

upon each of these a few grains of finely-powdered (artificial or precipitated) sulphate of baryta, moistened by a drop or two of water, and connect the discs (which should be within half an inch of each other) by a few filaments of wet cotton $a$. In a few minutes baryta will be apparent to testpapers at the negative disc, and sulphuric acid at the positive: they will mutually traverse the bridge of wet cotton, and if the decomposing agency be long enough continued, the whole of the baryta will be found on one side, and the acid on the other.

This experiment led Sir H. Davy to ascertain how far one substance might thus be electrically attracted through another, possessing a strong chemical attraction for it: the following is one of the means by which he effected this:-three small platinum trays, about an inch square, were placed side by side, and connected with each other by shreds of well-soaked cotton; care was taken to 
keep them upon a level. The exterior vessels were then filled with solution of sulphate of soda, and rendered negative and positive; and the central one with solution of ammonia, a highly alkaline liquid, having a strong attraction for sulphuric acid. It was found, after some hours' electrisation, that a large portion of the sulphuric acid of the salt had passed from the negative to the positive vessel ; that it had, therefore, traversed the ammoniacal solution, but had not there stopped in combination, as would have been the case had the substances not been under the electrical influence.

In some of his experiments on these electrical transferences, Sir H. Davy employed vessels consisting of the substance to be decomposed; the mineral called gypsum, for instance, or alabaster, is composed of sulphuric acid and lime: chemically speaking, it is a sulphate of lime. Two small cups of alabaster were filled with water, and united by moist cotton; they were rendered negative and positive by placing one pole of the pile in each cup ; the negative cup soon was found to contain a solution of lime, and the positive cup free sulphuric acid.

In some experiments thus conducted, where distilled water was employed and vessels of glass, a considerable quantity of acid and alkaline matter was elicited; this was afterwards traced to the decomposition of the glass, which contains alkali, and is not free from foreign salts: to avoid this source of error, therefore, he electrised distilled water in two vessels of gold, properly connected, thus employing a material which could not furnish 
impurities to the water; still, however, alkali and acid made their appearance. This evolution of foreign matters by the electrisation of apparently pure water, had been observed by others ; and their appearance was, by some, referred to a power supposed to be possessed by electricity, of generating the matters evolved; it was even imagined that water might possibly be a simple or elementary body, capable of forming an acid in union with positive electricity, and an alkali with negative electricity. In reviewing these and other opinions, it occurred to Davy, that the water, although carefully distilled, and apparently pure when examined by common tests, might possibly contain minute portions of foreign matter, rendered evident by the superior searching and decomposing power of electricity. He accordingly carefully redistilled the water at a low temperature in silver vessels, and on employing it in the above experiments, thus redistilled, it was found to afford much less indication of impurity; but even after all these precautions, acid and alkali were sparingly evolved from it. Of this impurity, part was traced to the accidental contact of the hands, with the apparatus used, the perspirable matter leaving traces of common salt; and now, aware of this source of contamination, and carefully avoiding it, there were scarcely any traces of acid or alkali to be observed. These extraneous elements, however, notwithstanding all the precautions above alluded to, were still perceptible after the process of electrical decomposition had been carried on for a length of time, and the only probable source whence they could be derived was the atmosphere, 
the elements of which, by uniting chemically with those of water, might give rise to the formation of nitric acid and ammonia. This chemical action or combination, Davy thought, might possibly be brought about by electric agency: accordingly, in addition to all the previous precautions, he now conducted the experiments in the exhausted receiver of a good air-pump, and had the satisfaction to find that, under such circumstances of the careful exclusion of all foreign agents, the water was resolved into its own elements, oxygen and hydrogen, only; and, consequently, that there were no grounds for supposing that any new forms of matter were producible by the union of electricity with other bodies.

Another important inference was deduced from the above, and many similar experiments; it was obvious that electricity had the power of searching out and separating the minutest portions of foreign matter, and of overcoming the most powerful chemical attractions; might it not, therefore, when properly applied to different bodies, indicate the existence of substances hitherto unsuspected, and even lead us to the knowledge of new elementary substances? Proceeding upon such grounds, Sir H. Davy submitted the fixed alkalies, which were considered at that time as simple or elementary bodies, to the decomposing agency of the pile, and was fortunate enough in this, his first trial, to obtain from them new elements : at the positive pole oxygen was evolved from them, but brilliant metallic globules appeared at the negative pole, extremely inflammable, and which were shewn to be the bases of those bodies. The 
names potassium and sodium were given to these new and singular metals; and by analogically applying the phenomena of their decomposition to that of the earths, also then on the list of elements, they also afforded evidence of decomposition, and like the alkalies, were shewn to be combinations of peculiar metals with oxygen. Many other discoveries have been the result of this new mode of research; but these, as well as the above, will be more fully dwelt upon afterwards.

It has been shewn, that when compound bodies are subjected to electrical decomposition, their elements are attracted with uniform phenomena by the respective poles; that certain substances are repelled by the negative, and attracted by the positive pole, and vice versâ; and upon this principle it was suggested as probable, that the inherent electrical states of the bodies were dissimilar to those of the pole by which they were attracted. Hence the elementary bodies may he arranged under two comprehensive classes-electronegative elements, and eloctropositive elements. In electrical decompositions the former will of course be attracted by the positive, and the latter by the negative pole.

In the present state of our chemical knowledge, the elementary or simple bodies may be regarded as about fifty-two in number: of these, five are usually termed supporters of combustion, seven are non-metallic combustibles, and the remaining forty are metals, or substances of which we hitherto know too little to enable us to separate them from that class. 
The five former bodies only, are electronegative; that is, when their compounds are subjected to electric decomposition, they make their appearance at the positive pole: the next seven are attracted by the negative pole, and are, therefore, presumed to be electropositive; the metals are in the same predicament. The undecomposed substances, therefore, arranged in reference to this view, will stand as follows:

Electronegative bodies attracted by positive surfaces.

Oxygen.

Chlorine.

Iodine.

Bromine.

Fluorine.
Electropositive bodies attracted by negative surfaces.

Hydrogen. Nitrogen. Sulphur. Phosphorus. Selenium. Carbon. Boron. The metals.

In relation to each other, however, the above substances may indicate opposite electricities, which will influence their appearance at the voltaic poles. We have seen that when oxygen and hydrogen are combined, as in water, the compound is uniformly so decomposed, that the whole of the oxygen is evolved at the positive pole, and the whole of the hydrogen at the negative; so in the decomposition of a compound of iodine and hydrogen, the iodine appears at the positive pole. But, when a compound of oxygen and iodine is electrically decomposed, the iodine collects at the negative wire, and hence, considered abstractedly in relation to oxygen, would be called an electropositive element. So, also, when a compound 
of hydrogen and sulphur is electrically decomposed, the sulphur appears at the positive pole; and hence, in respect to hydrogen, sulphur is an electronegative element.

The decomposition of water shews the transit of oxygen to the positive pole (see p. 237); that of the other electronegatives may be exhibited as follows; and for these, and many other of the experiments of decomposition, the annexed form of apparatus will be found useful. It is a cell of

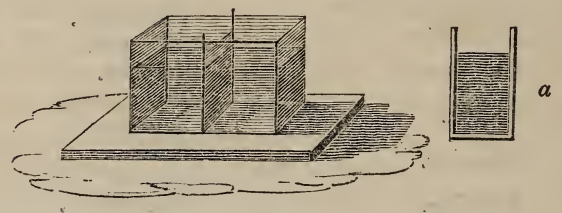

plate-glass, made by cementing four pieces together, with transparent varnish, and supporting them upon a wooden foot, into which they are fastened with putty. The cell is about five or six inches long, and about an inch broad, and may be divided into two parts, by the insertion of the temporary diaphragm $a$, which is a small frame of cane with muslin stretched over it. When this is in its place, a separate pole of the battery may be introduced on each side of it: the poles may most conveniently consist of two pieces of thin platinum, about four inches long and half an inch broad.

To shew the evolution of chlorine at the positive pole, fill the glass cell with weak salt and water, acidulated by muriatic acid, and coloured 
blue by the addition of a few drops of a sulphuric solution of indigo; then introduce the poles of the pile, and in a few minutes the positive division will begin to lose colour, and at length become colourless, in consequence of the evolution of chlorine derived from the acid and salt: here, the presence of chlorine is rendered evident by its bleaching power, which destroys the blue of the indigo.

The presence of uncombined iodine is announced by its property of striking a deep blue colour with a solution of starch. To demonstrate its electro-polarity, therefore, fill the cell with a very weak solution of starch, to which a little common salt and iodide of potassium have been added; then electrise as before, and the iodine will shew itself on the positive side by a beautiful blue colour.

The presence of bromine is announced in the same way by its yellow colour; and of fuorine by its action on glass.

The electropositive bodies, on the other hand, are evolved from their combinations with the electronegatives, at the negative pole, as is seen in the case of the decomposition of water, \&c. There are many striking experiments shewing the attraction of the metals by negatively electrified surfaces ; and, indeed, whenever solutions of metallic salts are electrically decomposed, their precipitation at the negative pole is more or less perfectly observed, according to their facilities of reduction, or deoxidizement. A plate of silver, for instance, when inmersed into a dilute solution of blue vitriol (sulphate of copper), occasions no 
change; but if the silver be rendered electronegative by communication with the negative pole of the battery, a precipitation of metallic copper immediately ensues. In the same way solutions of lead, tin, \&c. may be decomposed by the attractive energy of electronegative surfaces, and by metals which, under common circumstances, do not effect their decomposition.

When, as in the preceding case, the metals are rendered electronegative in the voltaic current, they are at the same time rendered attractive of each other, and of the electropositive bodies in general: thus, under such circumstances, the earths and oxides, and the inflammables generally, will be attracted by, or precipitated upon them; but the same cause which renders them attractive of these bodies, renders them repulsive of the electronegative class, among which are the bodies chiefly characterized by their corrosive and solvent action upon them. A piece of iron, for instance, rendered electronegative, will remain bright and clean in water, which, under ordinary circumstances of immersion, would rust and corrode it ; but if the iron be rendered electropositive, it then corrodes much more rapidly than in plain water; that is, it is then rendered attractive of oxygen, acids, and the other solvent agents, of which, when electronegative, it is repulsive.

These facts led Sir H. Davy to infer, that certain metallic substances which, under ordinary circumstances, are not acted upon by water and acids, might be rendered active by communicating to them the positively electrical state; and, on the contrary, that such metals as are easily acted 
upon by the above-mentioned agents, might be rendered inert in regard to them, by giving them the negatively electrical state. This, indeed, is merely another illustration of the fact, that bodies dissimilarly electrified attract, and similarly electrified repel each other. The following are illustrative experiments :-

Silver is a metal which is not acted upon by pure water, nor by salt and water, nor by dilute muriatic acid. But, let the poles of the battery consist of two strips of silver, and immerse these in water; it will then be found that the negative pole remains bright and clean, and is not in the least acted upon: but with the positive pole the case is very different; it becomes discoloured, and a white cloud collects about it in the water, which is oxide of silver; here, the silver is rendered attractive of the oxygen of the water, and enters into combination with it.

If, for pure water, we substitute a very weak solution of common salt, or a very dilute muriatic acid, the negative silver will still remain bright, but the positive becomes encrusted with a white or grey powder, and is soon evidently corroded; this arises from its being rendered attractive of the chlorine contained in the salt and in the acid, and the new substance formed is a chloride of silver.

If we substitute for silver any more oxidizable metal, that is, any metal more readily acted upon by air, water, and acids, the effects are still more striking, and we then have a better opportunity of observing the utter non-action of the negative surface, as opposed to the increased activity of 
the positive. For instance: for the silver poles of the preceding experiment, substitute two polished plates of iron, and immerse them, when connected with the pile, into a weak sulphuric or muriatic acid, or into a solution of salt-liquids which, under ordinary circumstances, act upon and corrode iron; the negative plate will now remain bright, but the positive will be very energetically acted on, and rapidly oxidized and dissolved.

This experiment may be varied and rendered more striking, as follows :- Fill the glass cell described at p. 248, with a weak solution of common salt in distilled water, to which a few drops of ferrocyanate of potassa * have been added, and immerse one of the iron plates in each partition; the negative side will remain unchanged, but the production of a deep blue colour on the positive side, will presently announce to the eye the rapid action upon the iron that is there going on. If we substitute a little infusion or tincture of galls for the ferrocyanate, the solubility of the iron is then shewn by a black tint $\dagger$.

* This salt is commonly called prussiate of potassa, and is a most delicate test of the presence of iron in solution, which it shews by the production of a more or less intense and copious cloud of blue. This blue precipitate is a ferrocyanate of iron, or Prussian blue.

$\uparrow$ Infusion of galls is another excellent test of the presence of iron; it is made by infusing a bruised gall-nut in a quarter of a pint of boiling water; when cold, the infusion is filtered off, and on adding a few drops of it to any liquid containing iron, a purple or black cloud is immediately perceptible. Should the liquid which is suspected to contain iron be very acid, it should be neutralized by the addition of an alkali previous to the application of the test. 
The want of action of the negative pole is shewn in these experiments, and may be more explicitly illustrated by the following:-

Silver and copper are metals which are readily acted upon by dilute nitric acid; it dissolves them with effervescence; but if they are plunged into the acid when rendered electronegative, they resist its solvent or oxydizing power.

These cases, however, of the prevention of ordinary chemical action by the agency of electricity, admit of a more simple illustration, independent of the use of the pile. It has been stated above, that when a plate of zinc in the contact of one of copper is immersed into water, a voltaic or electric circuit is produced, the copper being rendered negative, and the zinc positive. Under these circumstances, therefore, although the zinc is more speedily corroded than it otherwise would be, the copper is proportionally protected, and resists those agents which otherwise would attack it. This prevention of chemical action may be further illustrated as follows: Dilute some nitric acid in a wine-glass with five or six parts of water, so that it may act with very moderate rapidity upon a piece of sheet-copper immersed into it; in the course of a few minutes the acid will have acquired a slight blue colour, from the presence of dissolved copper, which may be rendered more striking by dropping a little of it into a solution of ammonia*, when a deep blue colour

* Ammonia, or the volatile alkali, is a most efficacious test of the presence of copper, and as that pernicious metal is often contained in preserves, pickles, and occasionally in other articles of food, it may be usefully resorted to for the 


\section{ELECTRO-CHEMICAL ACTION APPLIED TO}

will immediately announce the presence of copper: but if the copper-plate be united with one of zinc, and then plunged into the same acid, it will be found not to have been acted on, the zinc only being dissolved.

Again: immerse a polished plate of iron into a glass of water impregnated with carbonic acid (soda-water does very well); and in a few hours the water will be discoloured, and a portion of the iron dissolved, as may be shewn by the black colour produced on adding a little infusion of galls. Now, tie a strip of zinc in close contact with the iron, and immerse the two metals into the same water: after the lapse of some days, none of the iron will have been taken up; indeed, it will remain as bright as when first put in.

These examples, which might be numerously multiplied, furnish sufficient evidence of the curious interference of the electrical with the chemical powers of matter. Sir H. Davy, to whom we owe the discovery or development of the principal facts which they establish, aimed at a very

purpose of detecting it: it is only necessary to add ammonia, in slight excess, to the suspected liquid, and if copper be present, a more or less intense blue colour will be produced, the liquid generally remaining clear. Another valuable test of the presence of copper in any liquid is the ferrocyanate of potassa, mentioned above, for the detection of iron: in solutions of copper it occasions a cloud or precipitate of a very characteristic rich brown tint. If a larger quantity of copper be contained in a liquid, it will be thrown down in a metallic state upon a piece of clean iron : the author has known the green ornaments upon a twelfth cake to contain so large a quantity of copper, as to leave a copper streak upon the blade of the knife with which they were cut through. 
THE PROTECTION OF COPPER SHEATHING. 255

important practical application of them, as a means of preventing the corrosion of the coppersheathing of ships.

It was generally believed that sea-water had but very little action upon pure and well-rolled copper, and that its occasional rapid corrosion depended upon some impurity of the metal, or some imperfection in its manufacture. It was found, however, on immersing pieces of the best and purest copper that could be procured, in seawater, that it soon became covered with a green powder, and was ultimately more or less oxidized and corroded. It was evident that this action depended upon the mutual attraction of the positive copper and the negative oxygen or acid contained in the water, and that it might, therefore, possibly be suspended or prevented; by communicating a negative energy to the copper, which would cause it to repel its assailants; and this was easily effected, as the previously detailed experiments teach us, by attaching to it any more oxidable metal, such as zinc, iron, or tin.

In experiments upon the small scale, it was found that a piece of unprotected copper immersed in a glass of sea-water, was soon covered by a green powder, part of which was dissolved, and part precipitated; but on attaching to it a piece of zinc, and keeping the metal, thus protected, under sea-water, it remained bright, and even untarnished for several weeks : of the facility, therefore, of thus effectually protecting the copper, there could be no doubt: but a point of great importance in reference to the present question was to determine the relative proportion which it 
was necessary for the protecting metal to bear to the protected. When the former was zinc or iron, and amounted to from one-fortieth to one onehundred-and-fiftieth of the copper surface, the latter metal underwent no change or decay; when the zinc was reduced to one two-hundredth, and one four-hundredth, there was some perceptible loss ; but it is, to a considerable extent, effectual, where it only amounts to one one-thousandth. For these purposes cast-iron answers extremely well, and is cheaper and more durable than zinc; for it will be remembered, that the protecting metal is acted upon with a rapidity directly proportional to its saving power.

As far, therefore, as protection, or prevention of decay is concerned, the above experiments are extremely satisfactory in their results, and seemed, at first sight, to hold out well-founded hopes of their being applicable upon the large scale; but it unfortunately happens that, when a negative electric power is thus conferred upon the copper, so as to protect it from oxidizing and corroding agencies, it becomes, as above explained, attractive of metals and of their oxides, and, in short, of the whole list of electropositive bodies; among these are the earthy substances contained in seawater, such as lime, magnesia, and some of their compounds: these, therefore, when the plan was adopted upon a large scale, and a protected vessel sent to sea, were precipitated or deposited upon the copper. Now it seemed, at first, as if this earthy coating would merely act as an additional preservative of the copper; but, unfortunately, the adventitious crust or surface thus formed, is 
most favourable to the adhesion of weeds, and of certain marine animals and insects; and these accordingly attached themselves to it so rapidly, and in such abundance, as to render the bottom extremely foul, and to interfere with, and impede the sailing of the vessel. Theory would lead us to expect, that the zinc or iron might be so exactly proportioned to the surface of the copper, as effectually to protect it, without at the same time communicating to it that tendency to attract an earthy crust, which has just been mentioned ; but in practice this has been found scarcely attainable. Sir H. Davy's beautiful and philosophical suggestion, therefore, has not, in consequence, been adopted.

It is indeed to be regretted that the successful applications of scientific reasoning and philosophical discovery to practical purposes, and to the arts of life, are rarely made by their original inventors; and the case before us threatens to turn out a painful instance of this remark, for a patent has been taken out for covering ships with iron plate, protected by zinc: the iron is said to be thus rendered comparatively durable and permanent, and not to have that tendency to become foul, by a little over protection, which belongs to copper ; whilst its extreme cheapness, in comparison with copper, adds greatly to the value of its application.

The above is a summary view of the effects of voltaic electricity as a chemical agent; and it would appear that, as such, it acts in some cases merely as an agent producing heat, and in others it manifests a peculiar decomposing action, and 
attracts the elements according to certain laws; at the respective poles. It deserves attention, that in the latter cases the presence of water appears an essential requisite, and the elementary bodies are separated from each other associated with its oxygen and its hydrogen, according to the statement at p. 239.

\$12.-Of the Identity of the different Forms of Electricity.

The identity of common and of voltaic electricity has sometimes been doubted, in consequence of the difficulty of effecting, by the former, those chemical changes and decompositions which the latter easily produces; but Dr. Wollaston* has shewn, that if means be taken to assimilate these two states of electricity, they produce similar effects. Two very fine platinum wires were inserted in capillary tubes; one extremity of each wire was pointed as fine as possible, and being introduced within the capillary tube to a short distance from its end, the glass was softened by heat, till it adhered to, and covered the point; that end of the tube was then gradually ground away, until the point of the wire was perceptible through a lens. The tubes, thus prepared, were introduced into a vessel of water, so that the metallic points were near each other, and formed an interrupted metallic circuit. One of the wires was connected with the ground, and the other with an insulated ball placed within a short distance of the positive conductor. When a current of

* 'Philosophical Transactions,' vol. xci. p. 427. 
sparks is thus passed through the water, bubbles of gas gradually issue from the wires, which may be collected in an inverted tube, and are found to be the mixture of oxygen and hydrogen in due proportions to form water (see page 237). This decomposition is very slow compared with that attained by voltaic electricity, as already described: its rapidity was found proportional to the smallness of the wire through which the electricity passed, and the dispersion of the electricity was prevented by the glass coating of the tube, which limited the escape or passage to the small uncovered points.

Other chemical decompositions were in the same way effected by common electricity: the current was transmitted, by two fine gold points, along the surface of a moistened card tinged blue by litmus; after a few turns of the machine, the blue was changed to red in the vicinity of the positive wire. The negative wire was then placed upon the red spot, and the original blue was thus soon restored. Here, therefore, an acid was evolved at the positive pole, and an alkali at the negative.

Two silver wires, coated with sealing-wax, so that their ends only were exposed, were inserted into a solution of copper; on transmitting a current of sparks from one wire to the other, the receiving or negative wire became coated with copper; and the copper coating was removed when the electricities were reversed.

These experiments, then, are sufficient to shew that common electricity, as it is usually called, namely, that excited by the electrical machine, 
possesses the same decomposing powers over the chemical constitution of matter, as that generated in the voltaic pile. As far also as heating power is concerned, these two electricities may be shewn to be identical; for the common electric spark produces intense heat, as is proved by the facility with which it sets fire to the vapour of ether or alcohol. The simplest way of making this experiment is to receive the spark into a small metal cup or spoon, containing a little spirit of wine warmed by previously inflaming it, and blowing it out: the spark immediately rekindles it. In the same way we apply the electric spark to various chemical purposes, and especially to effect the union and decomposition of various gaseous bodies. The facility with which hydrogen or inflammable air is thus ignited, has given rise to a very elegant and ingenious application of electricity to the purpose of obtaining artificial light. This form of apparatus, and other practical applications of electricity, will be explained in a following volume.

Such being the heat produced by the passage of the electric spark, it is often asked why it can be received with apparent impunity upon the hand, where it might be expected to shew its temperature by producing a blister. But in this case, the application of heat is too instantaneous to produce such effects ; just as we can touch a red-hot poker, provided the contact be merely momentary.

When the discharge of a large Leyden jar, or of several united, is made to pass through combustibles, an effect of heat is produced proportionate to the electric accumulation: in this way fine metallic wires may be burned, with very beautiful 
phenomena, especially by burning them stretched over a piece of white card or writing paper.

There is considerable difficulty, in this way, in inflaming gunpowder, which requires to be pulverized and rammed into a quill or a small cartridge-case ; if the discharge of a large jar, or battery, be then passed between two wires inserted in the centre of the squib, so as nearly to touch, it usually explodes. In attempting thus to inflame loose gunpowder, it is generally dispersed by the electric explosion, without inflammation; but it may be easily ignited by the following contrivance:-place the powder in a small ivory mortar, of which a section is here represented, and discharge a moderate sized Leyden jar through it, so that the spark may pass bətween the interrupted wires $a a$; in this case the powder will be dispersed, and not ignited.

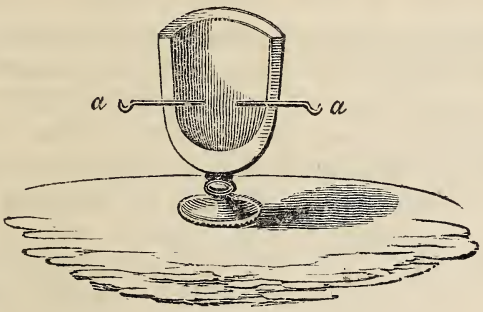

Now, repeat the experiment, placing a basin of water in the circuit, so that it may be interrupted by ten or twelve inches of that fluid, and the spark that passes under such circumstances will invari- 
ably fire the powder; or, the circuit may be interrupted by a few inches of packthread well soaked in water, and the same effect will ensue. The following is a representation of the arrangement: $a$ is a Leyden jar with a piece of chain

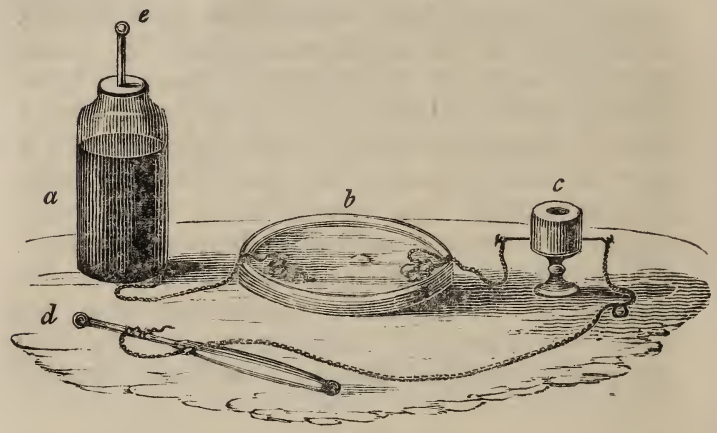

touching its exterior coating, and immersed at the other end into the shallow dish of water $b$, from the opposite side of which another chain issues, attached to one of the conductors of the mortar $c$ : a chain is also attached to the opposite conductor; and the discharge is effected, by bringing the ball of the discharger $d$, in contact with the knob of the jar $e$. It would appear probable that when the circuit is entirely metallic, the passage of the electricity is too rapid to kindle the powder, but that it does so when retarded somewhat in its progress, by being made to traverse the water.

A curious experiment, which may be called 
electro-chemical, consists in tracing lines or devices upon a thin plate of glass, covered with lac varnish, with the knob of a positively charged Leyden phial ; positive electricity is thus communicated to the side touched, and corresponding lines of negative electricity are produced by induction, upon the opposite side. If we now suspend the glass plate insulated in the air, and blow upon it a finely-powdered mixture of sulphur and red lead, the latter only will adhere to those parts of the plate which are positively electrified. If we now blow the same mixture upon the other side of the plate, the sulphur alone will adhere to the parts which are negatively electrified. Thus the same lines will be traced upon both sides of the glass plate, but with different coloured powders, the positive lines being red and the negative yellow. The cause of this appears to be, that when sulphur and red lead are triturated together, the former acquires positive and the latter negative electricity: hence the respective powders attach themselves to those surfaces which have an electricity opposed to their own.

\$ 13.- Of the Source of the Electricity of the Voltaic Apparatus.

The theory of the Voltaic pile is involved in many difficulties; but the original source of electricity appears to depend upon the contact of the metals, for, as we have already shewn, a plate of silver and a plate of zinc, or of any other difficultly and easily oxidable metals, become negative and positive on contact. The accumulation 
must, in part, be referred to induction, which takes place, in the electrical column, through the very thin stratum of air or paper; and through water, when that fluid is interposed between the plates. Accordingly we observe that the apparatus is in the condition of the series of conductors with interposed air; and of the Leyden phial. When the electric column is insulated, the extremities exhibit feeble negative and positive powers; but if either extremity be connected with the ground, the electricity of the opposite pole or extremity is greatly increased, as may be shewn by the increased divergence of the leaves of the electrometer which then ensues.

Dr. Wollaston, and some other eminent philosophers, have contended that chemical action is the source of electricity both in the common machine, and in the pile (Phil. Trans. 1800); but this opinion has been opposed, and I think successfully, by Sir H. Davy (Elements of Chem. Phil. 163). Zinc and copper, he observes, different metals and oxalic acid, different metals and sulphur, or charcoal, exhibit electrical effects after mere contact, and that, in cases when not the slightest chemical change can be observed; and if in these experiments chemical phenomena are produced by the action of heat or liquids, all electrical effects immediately cease; and it is not philosophical to assume a cause to account for an effect, when no such cause can be perceived.

It has been supposed that the action of the common electrical machine depends upon the oxidation of the amalgam; but Sir Humphry found, by mounting a small machine in a glass vessel, in 
such a manner that it could be made to revolve in any species of gas, that it was active in hydrogen gas, and more active in carbonic acid gas than in the atmosphere (probably owing to its greater density). The experiment was several times repeated under different circumstances, and uniformly with the same results; and may be regarded as decisive in this important question, for neither hydrogen nor carbonic acid could possibly oxidize the amalgam.

Sir H. Davy further observes, that electrical effects are exhibited by the same bodies, when acting as masses, which produce chemical phenomena when acting by their particles; it is not, therefore, improbable, that the primary cause of both may be the same, and that the same arrangements of matter, or the same attractive powers, which place bodies in the relations of positive and negative, $i$. $e$. which render them attractive of each other electrically, and capable of communicating attractive powers to other matter, may likewise render their particles attractive, and enable them to combine, when they have full freedom of motion.

It is not a little in favour of this hypothesis, that heat, and sometimes heat and light, result from the exertion of both electrical and chemical attractive powers; and that by rendering bodies, which, on contact, are in the relation of positive to others, still more highly positive, as has been above stated, their powers of combination are increased (page 250); whereas, when they are placed in a state corresponding to the negative electrical state, their powers of union are destroyed (page 
251). That acids can be detached from alkalies, oxygen and chlorine from inflammable matter by metallic substances, or by a fluid menstruum highly positive, is likewise favourable to the supposition.

This view of the possibility of the dependance of electrical and chemical action upon the same cause, has been much misrepresented. It has been supposed that the idea was entertained, that chemical changes were occasioned by electrical changes; than which nothing is further from the hypothesis which has been advanced. They are conceived, on the contrary, to be distinct phenomena; but produced by the same power, acting in one case on masses, in the other case on particles. The hypothesis has been attempted to be controverted by experiments which are far from satisfactory, and some of which have no connexion with it. It has been said, that acids rendered positive by the common machine, will still combine with alkalies, and that other contradictory results may be obtained; but a non-conducting acid, though brought in contact with a positive surface, electrified by the common machine, is not rendered positive throughout; but gains a polar electricity, which extends only to a certain depth into the crystals, and the exterior surface, if electrical at all, is negative; and if a wire, positively electrified by the common machine, be introduced into an acid solution, this solution, if at all affected, when made to act upon another solution, will be negative at its point of action ; that is, it will be positive near the wire, but will be in the opposite state with regard to another surface. And 
common electricity is too small in quantity, in its usual form of application, to influence chemical changes ; for it requires a very strong machine acting upon a very small surface, to produce any sensible polar decompositions of bodies, as has been above explained.

The power of action of the Voltaic apparatus, seems to depend upon causes similar to those which produce the accumulation in the Leyden battery, namely, the property of non-conductors and imperfect conductors to receive electrical polarities from, and to communicate them to conductors; but its permanent action is connected with the decomposition of the chemical liquids between the plates. Each plate of zinc is made positive, and each plate of copper negative, by contact; and all the plates are so arranged with respect to each other as to have their electricities exalted by induction, so that every single polar arrangement heightens the electricity of every other polar arrangement; and the accumulation of power increases with the number of the series. When the battery is connected in a circle, the effects are demonstrated by its constant exhibition of chemical agencies, and the powers exist as long as there is any menstruum to decompose: but when it is insulated, and the extreme poles of zinc and copper are unconnected, no effects whatever are perceived to take place, no chemical changes go on, and it exhibits its influence only by communicating very weak charges to the electrometer, the end terminated by zinc communicating a positive charge, that terminated by copper, a negative charge. 
That each plate of the most oxidable metal in the apparatus, is in the relation of positive, and each plate of the least oxidable, in the relation of negative, and that every series is possessed of similar and equal polarity, is shown by a very simple experiment: forty rods of zinc of the same size, connected with forty silver wires precisely similar, were introduced in the regular order into similar glasses filled with a solution of muriate of ammonia, rendered slightly acid by muriatic acid; as long as the extreme parts remained unconnected, no gas was disengaged from the silver, and the zinc was scarcely acted upon; when they were connected, all the plates of zinc were dissolved much more rapidly, and hydrogen gas was evolved from every silver wire. And in another experiment, in which several of these wires at equal distances were introduced into small glass tubes, it was found that equal quantities of hydrogen were produced.

Sir Humphry further remarks, that there are no fluids, except such as contain water, which are capable of being made the medium of connexion between the metals of the Voltaic apparatus, and refers the activity of De Luc's column to the moisture adhering to the paper discs ; to this view of the subject we have already adverted above.

\section{\$ 14.-The Theory of Natural Phenomena as connected with Electricity.}

Having now considered electricity in relation to its principal chemical effects, we may proceed briefly to examine its influence as a natural agent. 
Electricity is called into action, upon a grand and sublime scale, in the production of the thunder storm. It has been by no means satisfactorily ascertained to what the enormous electric accumulation that in such cases ensues, is to be attributed, but it is probably referable to a variety of causes, and connected with some peculiar power in masses of aërial vapour to receive and retain electrical charges, which do not admit of artificial imitation, and cannot, therefore, be satisfactorily investigated or explained by experiments upon a smaller scale.

The ancients referred the phenomena and effects of the thunder-storm to the collision and attrition of clouds and vapours, impelled and agitated by violent storms and currents in the higher regions of the atmosphere; and, in more modern times, before the discoveries in electricity which have been noticed, subtile inflammable and sulphureous exhalations were supposed to exist in the air, and, by their ignition and explosion, to produce those stupendous effects which we are about to describe.

At a very early period of electrical science, an analogy between the electric spark and lightning was suggested. Mr. Grey and Dr. Wall, and afterwards the Abbé Nollet *, hinted much more explicitly at the analogy. 'If any one,' says he, ' should take upon him to prove, from a well-connected comparison of phenomena, that thunder is in the hands of nature what electricity is in ours; that the wonders we now exhibit at pleasure are

* Leçons de Physique Expérimentale, tome iv. p. 314. Paris, 1748. 
small imitations of those great effects which alarm us, and that the whole depends upon the same kind of mechanism; should it be shown that a cloud, formed by the action of the winds, by heat, and by a mixture of various exhalations, is, when opposite to a terrestrial object, as an electrified body when at a certain distance from one that is not electrified, I confess, such an idea, if well supported, would afford me infinite pleasure, and, that it may be supported by many plausible arguments, is obvious to any one well versed in the history of electrical phenomena. The universality: of the electric matter, the rapidity of its action, its heat, and its activity in inflaming other bodies; its property of striking them externally and internally, even to their smallest parts ; the remarkable instance we have of this effect in the Leyden experiment; the notion which may be legitimately adopted of the effects that might be supposed to arise from a much greater accumulation of electric power: these, and many other points of analogy which I have for some time meditated upon, almost induce me to believe that, in taking. electricity as a foundation, one might form much more perfect and plausible hypotheses respecting the origin of thunder and lightning than any that have been hitherto suggested.'

Such are the ingenious suggestions of Nollet respecting the analogies between electricity and lightning, and their truth was afterwards amply proved by Franklin, who, like his predecessors, meditating upon the similarity of their effects, traced out further resemblances, and at length hit upon the happy expedient of sending up a com- 
mon kite to an electric cloud, and thus experimentally demonstrating their identity. The following are the particulars of this curious discovery *.

Franklin begins his account of the similarity of the electric fluid and lightning, by cautioning his readers not to be staggered at the great difference of effects in point of degree, since from that no. fair argument could be drawn, of the actual disparity of their nature. It is, he says, no wonder that the effects of the one should so far exceed those of the other ; for if two gun-barrels electrified will strike at two inches distance, and make a report, at how great a distance 10,000 acres of electric cloud must strike and give its fire, and how loud must be the crash. He then adds, that flashes of lightning are generally crooked and waving, and so is a long electric spark; that lightning, like common electricity, strikes the highest and most pointed objects in its way, in preference to others, such as hills, trees, towers, spires, masts of ships, points of spears, \&c. ; that it takes the readiest and best conductor; that it sets fire to inflammable bodies, rends others to pieces, and melts the metals. Lightning, he adds, has often been known to strike people blind, and the same happened to a pigeon which had received a violent shock of electricity; in other cases it has killed animals, and they have also been killed by electricity.

Reasoning on these effects, and having observed that pointed conductors appear to attract electricity, he conceived that pointed rods of iron fixed 
in the air might draw from clouds their electric matter, without noise or danger, and dissipate it at their termination in the earth: the following is his memorandum upon this subject:- "The electric fluid is attracted by points; we do not know whether this property be in lightning, but since they agree in all particulars in which we can already compare them, it is not improbable that they agree likewise in this. Let the experiment be made.'

In the year 1752, while waiting for the erection of a spire in the city of Philadelphia, not imagining that a pointed rod of any moderate height would answer the purpose, it occurred to him, that, by means of a common kite, he might have ready access to the higher regions of the atmosphere. Preparing, therefore, a large silk handkerchief, and two cross sticks to extend it on, he took the opportunity of the first approaching thunder-storm, and went into a field, where there was a shed proper for his purpose. But, dreading the ridicule which he feared might attend an unsuccessful attempt, he communicated his intention to no one but his son, who assisted him in flying the kite. A considerable time elapsed without appearance of success, and a promising cloud passed over the kite with no effect, when, just as he was beginning to despair, he observed some loose threads upon the string of the kite begin to diverge and stand erect; on this, he fastened a key to the string, and on presenting his knuckle to it, was gratified by the first electric spark that had thus been drawn from the clouds; others succeeded, and when the string had become wet 
by the falling rain, a copious stream of electric fire passed from the conductor to his hand. What were Franklin's emotions upon this interesting occasion, it is not difficult to conceive : we are told that when he saw the fibres of the string diverge, and the spark pass, 'he uttered a deep, sigh, and wished that the moment were his last;' he felt that his name would be immortalized by the discovery.

Dr. Franklin pursued these experiments with much assiduity and success: he erected an insulated iron rod to draw the lightning of the clouds into his house, and performed, with the electricity thus derived, nearly all the experiments for which he had before employed the common machine; and that no opportunity might be lost of making such experiments, he attached a chime of bells to the electric rod, which gave him notice, by their ringing, of the electric state of his apparatus.

In justice, however, to two French gentlemen, Messrs. Dalibard and Delor, we must remark, that they probably were the first who experimentally verified Franklin's hypothesis, although the Doctor was unacquainted with their proceedings. The former prepared his apparatus at Marly, near Paris ; the latter at his house, which stood upon high ground in that city. M. Dalibard's apparatus consisted of an iron rod forty feet long, the lower end of which was brought into a sentry-box where the rain could not enter, while on the outside it was fastened to three wooden posts by silken strings defended from the rain. This machine was the first that happened to be favoured by a visit from the etherial fire. 
M. Dalibard himself was from home, but in his absence had entrusted the care of his apparatus to one Coisier, a joiner, on whose courage and understanding he could depend : this person, having had all necessary instructions, was directed to call some of his neighbours, particularly the curate of the parish, whenever there should be any appearance of a thunder-storm; at length the event arrived, and on Wednesday, the 10th of May, 1752, between two and three in the afternoon, Coisier heard a loud clap of thunder; he immediately ran to the sentry-box, and, in the presence of the curate and several neighbours, drew sparks from the conductor. A few days afterwards a successful repetition of the experiment was made by $\mathbf{M}$. Delor at Paris.

These important and interesting experiments were of course repeated in almost every civilized country with variable success : in France a grand result was obtained by $M$. de Romas *. He had constructed a kite seven feet high, and three wide, which was raised to the height of 550 feet by a string, with a fine wire interwoven through its whole length, to render it a better conductor. On the 26th of August, 1756, sparks, or rather streams of light were darted from the string of this kite, of an inch in diameter, and ten feet long.

Considering the facility, and, at the same time, the danger of these experiments, it is curious that they have only in one instance been attended by a fatal result, namely, in the case of Professor

* Mem. des Sqqavans Etrangers, tome ii. 
Richman of Petersburgh. He had constructed an apparatus for experiments on atmospherical electricity, which was entirely insulated, and with no contrivance for discharging it when too strongly electrified. On the 6th of August, 1753, he was examining the electricity of this apparatus in company with a friend, and whilst attending to an experiment, his head accidentally approached the insulated rod, and a flash of lightning immediately passed from it through his body and deprived him of life. A red spot was produced upon his forehead, his shoe was burst open, and a part of his waistcoat singed; his companion was for some time rendered senseless; the door of the room was split and torn off its hinges.

The discovery of the identity of lightning and electricity has not been without its important practical results, among which we may especially enumerate the application of Conductors to buildings and ships, by which their safety during a thunderstorm is almost ensured. Franklin's original proposition for the purpose is that almost generally adopted; it consists in erecting a perfectly continuous metallic rod by the side of any building which it is intended to protect from the effects of lightning; the rod is to be pointed at each extremity, and extended above the highest part of the building at one extremity, the other penetrating deep into the earth, or in contact with water. In establishing this safeguard no other precautions are necessary than to have the top well and permanently pointed; and it is better of copper than iron, because the former does not so easily acquire a coating of rust, which, being a 
non-conductor, might possibly diminish its efficacy : it is sometimes well to subdivide the extremity
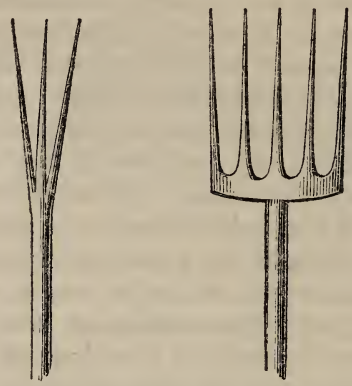

into three or four points : and it should be well secured to the side of the house in its descent, and penetrate so deep in the ground as always to be in free contact with a moist stratum of soil ; if it ends in a deep well, so much the better. The diameter of the rod should be such as amply to secure it against being melted by a sudden and copious flash of electricity, for which purpose half an inch is fully sufficient; and if the roof is leaded, a broad strip of lead should connect it with the conductor*. When a building is very extensive, it is as well to have a conductor at each end; and where a church spire is to be protected, all clamps and bars of metal of any magnitude used in its construction should have a metallic connection, by a strip of lead or otherwise, with the nearest

* It not unfrequently happens that the iron or leaden water-pipes attached to houses act as excellent conductors, and supersede the necessity of any specific arrangement. 
part of the conducting rod. A small building containing very dangerous materials, such, for instance, as a powder magazine, is perhaps most prudently defended from ignition by lightning, by placing the conductor within a few yards of the building, and elevated several feet above it; for this purpose it may be attached to a mast or signal post in the vicinity.

Conductors for ships have hitherto been made moveable, with the intention of occasionally attaching them to the mast during dangerous storms ; the consequence of which has been, that they have generally remained packed up below, and are not in their place in the time of danger: they are also generally made of chain, which is highly improper. We are lately indebted to Mr. Harris for drawing the attention of the proper authorities to this important subject; he judiciously recommends fixed conductors, and has experimentally demonstrated their safety and value.

Persons not otherwise nervous or timid, often experience particular sensations of alarm and discomfort upon the approach of a thunder-storm, arising from some constitutional peculiarity which perhaps renders them especially sensible of the influence of electricity upon the system. These and others are often led to inquire after the best means of safety upon such an occasion; the directions to be given are few and simple. If out of doors, trees should be avoided; and if, from the rapidity with which the explosion follows the flash, it should be evident that the electric clouds are near at hand, a recumbent posture upon the ground is the most secure. It is seldom 
dangerous to take shelter under sheds, carts, or low buildings, but it is better to avoid them. The distance of twenty or thirty feet from tall trees, or houses, is rather an eligible situation, for should a discharge take place, such prominent bodies are most likely to receive it, and the less elevated objects in their neighbourhood may therefore escape uninjured. It is also right during a thunder-storm to avoid rivers, ponds, and all streams of water, for they are good conductors, and the height of a human being when connected with them is likely to determine the course of the discharge. Within doors we are tolerably secure in the middle of a large carpeted room, or when standing upon a doubled hearthrug. We should avoid the chimney, for the iron of and about the grate, the soot that lines it, and the heated and rarefied air that it contains, are all conductors, and may tempt the lightning to descend by that channel: upon the same principle, gilt mouldings, bell-wires, and extensive metallic surfaces of any description, are also in danger of being struck. In bed we are comparatively safe, for feathers and blankets are bad conductors, and we are consequently, to a certain extent, insulated in such situations*.

The cellar has been sometimes recommended as a particularly safe place of resort, and so it

* When a tree is struck by lightning, it often happens that it is completely barked. This arises from the electricity taking that direction which best conducts it, and which is between the bark and the wood, where there is most sap and moisture. The bark on such occasions is often curiously spread round the tree in the form of a circle. 
often is; but we sometimes find that the basement story of a house is that which principally suffers: of this the cause is not very evident, though it is customary to attribute it to the lightning having proceeded from the earth to the clouds, instead of traversing in an opposite direction.

We are often told that there is no danger if a certain interval of time can be counted between the flash and the report of the thunder; this is true enough; indeed, if we can count at all, we are safe.

Where persons have been killed by lightning it has generally come upon them so suddenly as to leave no time for precaution; though it must also be admitted that some have suffered from foolishly ridiculing the idea of danger, and wantonly exposing themselves to the storm; walking out to admire its grandeur, or insisting upon opening the windows to look out and observe it. A severe thunder-storm is always an awful and dangerous phenomenon; and while, on the one hand, it is the extreme of folly to neglect common precautions, and to ridicule those that are alarmed, it is, on the other, equally weak, and even irreligious, to give way to unnecessary apprehensions and unjustifiable fears.

It is scarcely necessary here to describe a variety of amusing apparatus which has been constructed with a view to shew the effects of lightning upon buildings, the means in which it harmlessly traverses good and continuous conductors, and the mischief which it produces when they are insufficient or interrupted. Models of houses, powder magazines, and ships, properly fitted up for these 
demonstrations, are sold by the philosophicalinstrument makers.

The appearance of the heavens during a thunderstorm, the manner in which the clouds assemble, and attract and repel each other, the circumstance of their rising against the wind, and traversing the upper regions of the atmosphere in a variety of contrary directions, are phenomena which it is not difficult to explain by a reference to those general laws of electrical attraction and repulsion which have been already sufficiently explained. Upon the same principle we account for the singularly fringed appearance of a thunder-cloud, and for the ragged excrescences which it projects towards the earth, whilst its upper surface is generally smooth and well defined. We usually observe that a number of such clouds of small dimensions coalesce into a larger one, moving swiftly in all directions, and darting flashes from one to the other, whilst the wind rises and often blows in squalls; the lightning is more frequent as the clouds aggregate, and a great expanse of the heavens often appears in an almost continuous blaze of light. The thunder, at first rumbling in the distance, now approaches with various roaring and rattling sounds, and the rain often falls in torrents.

If we watch a severe thunder-storm from the beginning to the end, we shall observe a singular variety in the appearance of the flashes: the scene is sometimes rendered awfully magnificent by their brilliancy, frequency, and extent, darting sometimes in broad and well defined lines from cloud to cloud, and sometimes shooting towards the 
earth: they then become zig-zag and irregular, or appear as a large and rapidly moving ball of fire, an appearance usually designated by the uninformed, a thunderbolt, and erroneously supposed to be attended by the fall of a solid body. The report of the thunder is modified, according to a variety of circumstances, depending partly upon the situation of the observer, the nature of the country, and the extent of air which it traverses. Sometimes it sounds like the firing of many muskets at a short distance off; sometimes like the clattering of loose boards upon each other, or the sudden emptying of a large cartload of paving-stones: in these cases it usually quickly follows the lightning, and is near at hand: when more distant, it rumbles and reverberates, at first with a loud report, gradually dying away and returning at intervals, or roaring like the discharge of heavy artillery. In accounting for these phenomena it must be recollected, that the passage of electricity is infinitely rapid; a discharge through a circuit of four miles has been experimentally proved to be instantaneous; the motion of light is similarly rapid, and hence the flash appears momentary, however great the distance through which it passes. But with sound the case is very different; it is infinitely slower in its progress, moving only at the rate of about twelve miles in a minute. Now supposing the lightning to pass through a space of some miles, the explosion will be first heard from the point of the air agitated, nearest to the spectator; it will gradually come from the more distant parts of the course of the electricity; and last of all will be heard from 
the remote extremity ; and the different degrees of the agitation of the air, and likewise the difference of the distance, will account for the different intensities of the sound and its apparent reverberations and changes. If the observer be equidistant, or nearly so, from every part of the flash, he will hear a single crash: this will happen if we suppose $a$ in the cut to represent the observer, and $b$ the direction of the flash. But, if the observer be

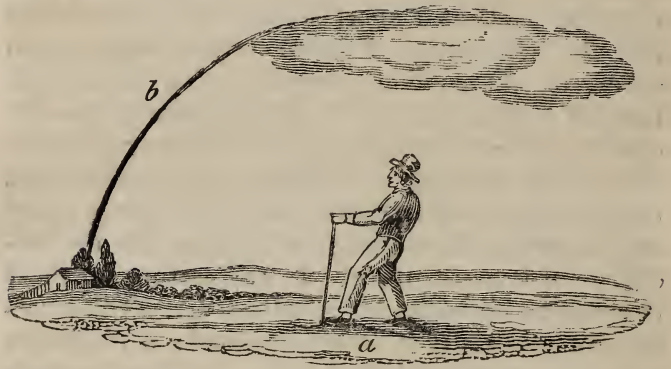

at $a$, the direction of the lightning being represented by the line $b$ (in the next page), the sound will first reach him from the nearest point, and regularly, but rapidly, die away as it recedes.

The common progress of lightning is, however, much less regular, and may be represented by the zig-zag line $c$, the spectator standing at $a$, in which case the sound will first come to him from the nearest point, and will then seem to recede and again to approach, rumbling irregularly as the sound arrives from the different portions of traversed air, some nearer and some further 


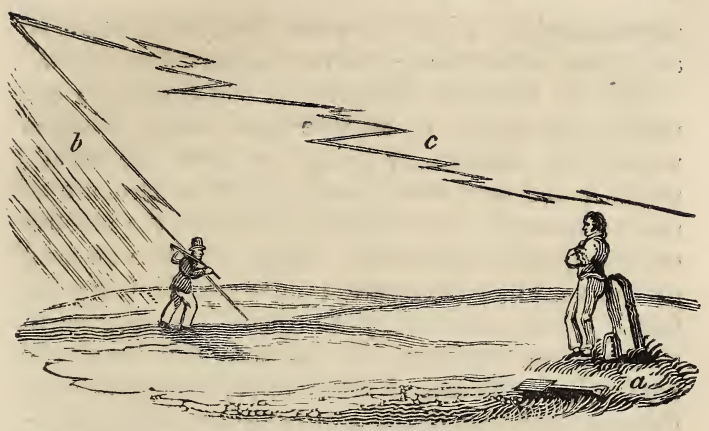

from him, until it is at length lost in the distance. This irregularity in the passage of the electric discharge is sometimes so considerable, that the thunder is actually lost to the ear at one moment and heard again at another, and this three or four times successively in the same clap.

There was at one time a foolish controversy among electricians respecting the relative advantages of points and balls in the construction of conductors; but, consistently with Dr. Franklin's original recommendation, points are now universally adopted. It has already been shewn that they slowly and silently discharge electricity, without admitting of induction, as is the case with balls, or large and equal surfaces. Dr. Franklin suggested an experiment in further proof of his opinion, which consisted in attaching to the prime conductor of the electrifying machine, one or more large flocks of cotton, so as to resemble electrified clouds. When a point is made to approach these, they collapse, recede, and quickly lose their electricity; when, on the other hand, they are 
approached by a ball, they are attracted towards it, and the electric charge is very slowly dissipated.

Of the cause of atmospheric electricity we are nearly ignorant: it is true that many changes which are continually goirg on upon the earth's surface, and in the surrounding atmosphere, are of such a nature as are known to disturb the electric equilibrium ; evaporation, change of state or form in the varieties of matter; changes of temperature ; chemical action; all these are sources of electrical excitation; but stili they can hardly be adduced as offering a satisfactory explanation of the enormous electrical accumulation that a thunder-storm evinces. That sudden, violent, and extensive chemical and mechanical changes in the forms and states of matter are causes by which electricity is often copiously manifested, is shewn by the flashes of lightning that accompany volcanic eruptions, and by the highly electric state of the surrounding atmosphere that attends them.

Another natural phenomenon, which may be referred to electric excitation, is the waterspout; it appears to result from the electric attraction of a mass of vapour or cloud acting upon the water beneath: it first causes the appearance of a hillock in the ocean; the water is then drawn up in a column towards the cloud, and the cloud and rain are attracted towards the water.

It occasionally happens that volcanoes suddenly break forth in the sea, and sometimes in very deep water; and they are attended by thunder, lightning, and waterspouts. Of such an eruption a curious account is given by Captain Tillard in the Philosophical Transactions, and of its general appearance and effects some notion may be formed 
from the following wood-cut. In June, 1811, Captain Tillard, on approaching the island of St. Michael, observed several columns of smoke rising in the horizon, which proved to issue from a marine volcano about a mile off the north-west end of the island, whither he proceeded more nearly to inspect the phenomena. ' Imagine,' he says, " an immense body of smoke rising from the sea, the surface of which was marked by the silver rippling of the waves occasioned by the slight and steady breezes incidental to those climates in summer. In a quiescent state it had the appearance of a circular cloud, revolving 'on the water like a horizontal wheel, in various and irregular involu-

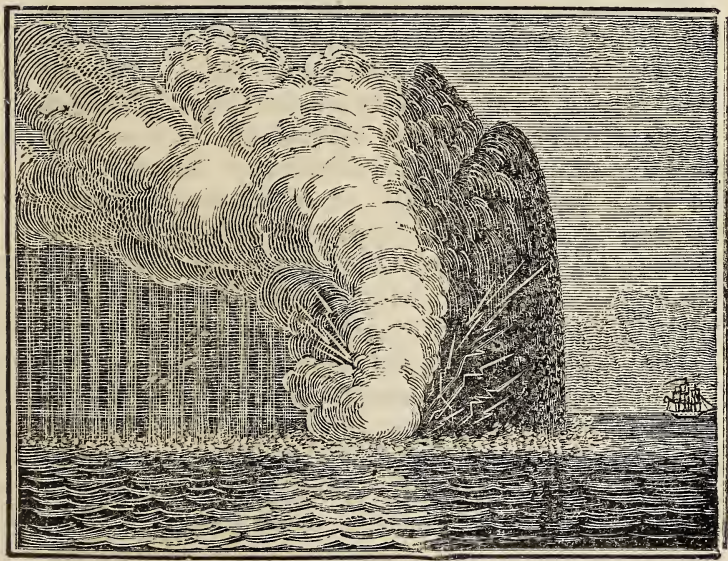

tions, expanding itself gradually on the lee side, when suddenly a column of the blackest cinders, ashes, and stones, would shoot up in the form of 
a spire, rapidly succeeded by others, each acquiring greater velocity, and breaking into various branches, resembling a group of pines; these again forming themselves into festoons of white feathery smoke. During these bursts, the most vivid flashes of lightning continually issued from the densest parts of the volcano, and the columns rolled off in large masses of fleecy clouds gradually expanding themselves before the wind in a direction nearly horizontal, and drawing up a quantity of waterspouts, which formed a striking addition to the scene. In less than an hour a peak was visible, and in three hours from the time of our arrival, the volcano then being four hours old, a crater was formed, twenty feet high, and from four to five hundred feet diameter.'

The account then goes on to state, that the eruptions were attended by a noise like the firing of cannon and of musketry intermixed, as also with shocks of earthquakes sufficient to throw down a large part of the cliff on which the observers stood; it is, however, principally to the lightning and to the waterspouts that I would here direct the reader's attention. Captain Tillard afterwards visited the volcanic island: it was eighty yards high; its crater upon the level of the sea was full of boiling water; it was about a mile in circumference, and composed of porous cinders and masses of stone.

The Aurora Borealis is another natural phenomenon, probably connected with or dependent upon electricity; its appearances indeed may be, to a certain extent, imitated by artificial electricity, which, when it passes through rarefied air, exhibits a diffused luminous stream, having much 
resemblance to the characteristic appearances of the northern lights. ' There is,' says Mr. Singer, - the same variety of colour and intensity; the same undulating motions, and occasional coruscations ; the streams exhibit the same diversity of character, divided at one moment into minute ramifications, and at another beaming forth in one body of light, or passing in distinct broad flashes; and when the rarefaction is considerable, various parts of the stream assume that peculiar glowing colour which occasionally appears in the atmosphere, and is regarded by the uninformed. observer with astonishment and fear.'

The affections of the magnetic needle and of the electrometer during the appearance of the Aurora, as well as other phenomena connected with it, have been noticed by several eminent philosophers. Halley and Dalton directed the attention of meteorologists to this subject; and more lately, Captain Franklin has added material information upon it*; it has also been accurately described by Captain Parry, who had ample opportunities of observing it in great perfection and splendour, during his perilous voyages in search of a north-west passage from the Atlantic to the Pacific.

In this country the Aurora Borealis has of late years been rarely observed. On the 13th of October, 1792, it appeared in great brilliancy; and an account of it, from which the following extract is taken, has been given by Mr. Dalton in his $M e-$ teorological Essays. The reader may also consult the description given by Mr. Kendall $\dagger$ of the Aurora which appeared here in October, 1829.

* Journey to the Shores of the Polar Sea, Appendix. + Quarterly Journal. 
' Attention was first excited,' says Mr. Dalton, ' by a remarkably red appearance of the clouds to the south, which afforded sufficient light to read by at eight o'clock in the evening, though there was no moon nor light in the north.

- From half past nine to ten there was a large, luminous, horizontal arch to the southward, and several faint concentric arches northward. It was particularly noticed, that all the arches seemed exactly bisected by the plane of the magnetic meridian. At half past ten o'clock, streamers appeared very low in the south-east, running to and fro from west to east; they increased in number, and began to approach the zenith, apparently with an accelerated velocity; when, all on a sudden, the whole hemisphere was covered with them, and exhibited such an appearance as surpasses all description. The intensity of the light, the prodigious number and volatility of the beams, the grand intermixture of all the prismatic colours in their utmost splendour, variegating the glowing canopy with the most luxuriant and enchanting scenery, afforded an awful, but, at the same time, the most pleasing and sublime spectacle in nature. Every one gazed with astonishment, but the uncommon grandeur of the scene only lasted about one minute; the variety of colours disappeared, and the beams lost their lateral motion, and were converted into the flashing radiations.

' Notwithstanding the suddenness of the effulgence at the breaking out of the Aurora, there was a remarkable regularity in the manner; apparently a ball of fire ran along from east to west with a velocity so great as to be barely distinguishable from one continued train, which kindled up the 
several rows of beams, one after another; these rows were situate before each other with the exactest order, so that the bases of each row formed a circle crossing the magnetic meridian at right angles: and the several circles rose one above another, so that those near the zenith appeared more distant from each other than those near the horizon, a certain indication that the real distances of the rows were nearly the same.'

This Aurora continued for several hours ; there were many meteors or falling stars, seen at the same time, but they appeared to be below, and unconnected with the Aurora.

During Captain Parry's perilous voyage, undertaken with a view of discovering a north-west passage from the Atlantic to the Pacific, he had numerous opportunities of witnessing the Aurora in uncommon magnificence. The following is an abstract of his description of its appearance on the 14th of December, 1821*.

- The Aurora began to shew itself as soon as it was dark. Innumerable streams of white and yellowish light occupied the heavens to the southward of the zenith, being much brighter in the south-east, from whence it often seemed to emanate. Some of these streams were in right lines, others crooked, and waving in all sorts of irregular figures, moving with inconceivable rapidity in various directions. Among them might frequently be observed shorter bundles of rays, which, moving even with greater velocity than the rest, have acquired the name of " merry

* Second Voyage for the Discovery of a North-West Passage, p. 142 . 
dancers." In a short time the Aurora extended itself over the zenith about half way down to the northern horizon, but no further, as if there was something in that quarter of the heavens which it did not dare to approach. About this time, however, some long streamers shot up from the horizon in the north-west, which soon disappeared. While the light extended over part of the northern heavens, there were a number of rays assuming a circular or radiated form near the zenith, and appearing to have a common centre near that point, from which they all diverged. The light of which these were composed appeared to have inconceivably rapid motion in itself, though the form it assumed, and the station it occupied in the heavens, underwent little or no change for perhaps a minute or more. This effect is a common one with the Aurora, and puts one in mind, as far as its motion alone is concerned, of a person holding a long ribbon by one end, and giving it an undulatory motion through its whole length, though its general position remains the same. When the streams or bands were crooked, the convolutions took place indifferently in all directions. The Aurora did not continue long to the north of the zenith, but remained as high as that point for more than an hour; after which, on the moon rising, it became more and more faint, and at half past eleven was no longer visible.

'The colour of the light was most frequently yellowish-white, sometimes greenish, and once or twice a lilac tinge was remarked, when several strata appeared as it were to overlay each other by very rapidly meeting, in which case the light 
was always increased in intensity. The electrometer was tried several times, and two compasses exposed upon the ice during the continuance of this Aurora, but neither was perceptibly affected by it. We listened attentively for any noise which might accompany it, but could hear none, but it was too cold to keep the ears uncovered very long at one time. The intensity of the light was something greater than that of the moon in her quarters. Of its dimming the stars there cannot be a doubt. We remarked it to be in this respect like drawing a gauze veil over the heavens in that part, the veil being most thick when two of the luminous sheets met and overlapped. The phenomenon had all the appearance of being full as near as many of the clouds commonly seen, but there were none of the latter to compare them with at the time.'

Our object in the above extracts has been to give an account of those appearances of the Aurora which seem to connect them with electricity, and though Captain Parry neither observed the electrometer nor the magnetic needle to be influenced, Captain Franklin* remarked. them both to be affected. Nairne, Cavallo, and others, have described a peculiar hissing noise as one of the accompaniments of the Aurora; and Dr. Halley, in his description of a remarkable Aurorat, ascribes its production to the same influence as that which produces magnetism; and Beccaria conceived the phenomena of magnetism to be dependent upon a circulation of the electric * Journey to the Shores of the Polar Sea, Appendix.

† Phil. Tran., vol. xxx. 
fluid from north to south, originating from several sources in the northern hemisphere.

All meteors were at one time considered as derived from electricity; but the showers of stones by which many of them are accompanied shew that they must often be ascribed to other sources. There can, however, be little doubt that what are termed falling stars are of electric origin. These vary a little in size and colour, moving in various directions, but chiefly appearing to shoot towards the earth. They are most common in those states of the atmosphere favourable to electric accumulation, especially on clear frosty nights, or when the sky is cloudless and the wind easterly; in the clear intervals of a showery evening, and on summer nights, when well defined clouds are floating in a serene atmosphere. They are also common during the prevalence of the Northern Lights, appearing then to be lower than the Aurora; and from their comparative brilliancy, probably moving through a more dense medium.

The appearance of a shooting star may be accurately imitated by discharging a Leyden jar through an imperfectly exhausted glass tube, or air-pump receiver. The tube should be capped with brass at both ends, and about three feet long and an inch in diameter; if too perfectly exhausted the spark passes in a divided and pale stream of light, but on admitting a little more air it traverses in the form of a bright flash or spark.

It appears from the best conducted experiments on atmospheric electricity, that in the usual state of the atmosphere it is generally positive, but that it often changes to negative upon the first 
appearance of rain, snow, or hail. The approach of clouds often influences the electricity of the conductor so as to produce alternations of the two electricities. There appears, as might be expected, to be a greater accumulation of electricity in the atmosphere during the presence of regular thunder-clouds, than at any other period; a driving fog accompanied by small rain, a fall of snow, and a smart shower on a hot day, are also attended. by powerful signs of electric excitation. Hot weather succeeding wet, or wet weather following a series of dry days, are also favourable circumstances for the generation of atmospheric electricity. The atmosphere is least electrical during the prevalence of north-easterly winds, and in that state which produces a disagreeable sensation of dryness and of cold without a corresponding depression of the thermometer. It has also been remarked that the usual positive electricity is weakest during the night; that it increases with the sun-rise; decreases toward the middle of the day, and again increases as the sun declines; it then again diminishes and remains feeble during the night: it would therefore seem probable that the electricity of the atmosphere is influenced by the same causes that modify the distribution of moisture.

\section{§ 15.-Of Animal Electricity.}

There are some remarkable instances of the generation of electricity in living animals, to whom the power seems to be given principally as a means of defence. Of these animals, the Tor- 
pedo or Electric ray, was noticed by the ancient writers on natural history: it inhabits the Mediterranean and North Seas; its weight, when full grown, is about eighteen or twenty pounds. When touched, it communicates a benumbing sensation, and by repeated contacts gives a series of electric shocks.

The Gymnotus, or Electric eel, was first described in $167 \%$, by M. Richer, who was commissioned by the French Academy to make some mathematical observations in Cayenne. It is a native of the warmer regions of Africa and America, inhabiting the larger rivers; especially those of Surinam. In Africa it chiefly occurs in the branches of the Senegal. These animals have been anatomically described by Mr. Hunter in the 63rd and 65th volumes of the Philosophical Transactions. Their electric organs consist of alternations of different substances, and are most abundantly supplied by nerves; their too frequent use is succeeded by debility and death. That these organs are not otherwise essential to the animals, is shown by their thriving after they have been removed.

The Silurus electricus is less perfectly known: it has been described by Broussonet under the name of Trembleur*.

Humboldt, in his Tableau Physique des Régions Equatoriales, \&c., has given some curious details respecting the electrical eel which inhabits the rivers and lakes of the low provinces of Venezuela and the Caraccas. It is met with most frequently

* Histoire de l'Académie Royale des Sciences, 1782. 
in the stagnant ponds dispersed at intervals over the plains which extend from the Oronoco to the Apure. The old road near Urutica has even been abandoned on account of the danger experienced in crossing a ford, where the mules were, from the effect of the shocks, often paralyzed and drowned. Even the angler sometimes receives a stroke conveyed along his rod and line. These eels are about six feet in length, and occasion a highly painful sensation, more resembling the effect of a blow on the head than the shock of a common electric discharge; a peculiarity of effect, referable perhaps to a great quantity of electricity of small intensity *.

* The following particulars given by Humboldt (Edinb. Review, vol. xvi. p. 250) are too curious to be here omitted.

' The Indians entertain such a dread of the Gymnotus, and show so much reluctance to approach it when alive and active, that Humboldt found extreme difficulty in procuring a few to serve as the subjects of experiment. For this express purpose he stopped some days on his journey across the Llanos to the river Apure, at the small town of Calaboze, in the neighbourhood of which he was informed that they were very numerous. But, though his landlord took the utmost pains to gratify his wish, he was constantly unsuccessful. At last he determined to proceed himself to the spot, and was conducted to a piece of shallow water, stagnant and muddy, but of the heat of seventy-nine degrees, surrounded by a rich vegetation of the great Indian fig-trees and odoriferous sensitive plants. Here he soon witnessed. a spectacle of the most novel and extraordinary kind: about thirty horses and mules were quickly collected from the adjacent savanuahs where they run half wild. These the Indians drove into the marsh. The gymnoti, roused from their slumbers by the noise and tumult, mount near the surface, and swimming like so many livid water serpents, briskly pursue the intruders, and gliding under their bellies, discharge through them the most violent and re- 
Those who are fond of supposing that electricity is an agent in certain processes of vitality, have referred, in favour of their hypothesis, to certain other cases of its production by arrangements of different organic substances. If the hind legs of a frog be placed upon a glass plate, and the crural nerve dissected out of one made to communicate with the other, it will be found, upon making occasional contacts with the remaining crural nerve, that the limbs of the animal will be agitated at each contact. Hence some physiologists suppose that electricity may be concerned in some of the most recondite phenomena of life, and experiments have been made tending to confer some probability on this idea.

6 The principle once established, that there may exist in the animal economy a power of determining the development of electric excitement capable of being transmitted along the nerves; and it being ascertained by numerous and decisive experiments, that the transmission of voltaic electricity along the nerves of even a dead animal

peated shocks. The horses, convulsed and terrified, their mane erect, and their eyes staring with pain and anguish, made unavailing struggles to escape. In less than five minutes two of them sunk under the water and were drowned. Victory seemed to declare for the electric eels. But their activity now began to relax. Fatigued by such expense of nervous energy, they shot their electric discharges with less frequency and effect. The surviving horses gradually recovered from the shocks, and became more composed and vigorous. In a quarter of an hour the gymnoti finally retired from the contest, and in such a state of languor and complete exhaustion, that they were easily dragged on shore by the help of small harpoons fastened to cords.' 
is sufficient to produce the most violent muscular action, it became an easy step to refer the origin of muscular motion in the living frame to a similar cause, and to look to the brain, a wonderfully constituted organ, for which no mode of action possessing the least plausibility had ever been devised, as the source of the required electrical power.'

Mr. Herschel ${ }^{*}$, from whom we have quoted the last paragraph, extends his ideas upon this subject in the following note. "If the brain be an electric pile, constantly in action, it may be conceived to discharge itself at regular intervals, when the tension of the electricity developed reaches a certain point, along the nerves which communicate with the heart, and thus to excite the pulsations of that organ. This idea is forcibly suggested by a view of that elegant apparatus, the dry pile of Deluc $\uparrow$, in which the successive accumulations of electricity are carried off by a suspended ball, which is kept, by the discharges, in a state of regular pulsation for any length of time. We have witnessed the action of such a pile maintained in this way for whole years, in the study of the above-named eminent philosopher. The same idea of the cause of the pulsation of the heart appears to have occurred to Dr. Arnott, and is mentioned in his useful and excellent book on Physics, to which, however, we are not indebted for the suggestion, it having occurred to us, independently, many years ago.'

* Preliminary Discourse on the Study of [Natural Philosophy, p. 342 .

+ See page 215. 
$\S 16 .-$ Of the Connexion of Electricty with Magnetism.

Certain analogies between the phenomena of electricity and those of magnetism are so obvious, as long to have led to the supposition of some intimate mutual relation existing between those powers of matter. The subject has lately received important elucidation from the researches of Oersted and others, and upon these has been founded a new and highly interesting department of science. The chief investigations connected with it belong rather to physical than chemical philosophy; but as they are susceptible of some applications to the inquiries of the chemist, it becomes necessary here to give such statement of them as may render them intelligible. The reader who may be desirous of historical details and references to authors, may advantageously consult Mr. Faraday's ' Historical Sketch of Electromagnetism,' Ann. of Phil., N. S., vol. ii.

A metallic wire which is transmitting an electric current, the connecting wire, for instance, between the extremes of the voltaic apparatus, if brought towards a magnetic needle, has the power of attracting and repelling it in obedience to certain laws.

If a magnetic needle be left to take its natural direction, and then a straight portion of the connecting wire be brought above and parallel to it, that end of the needle next the negative pole of the battery moves towards the west, and that whether the wire be on the one or other side of the needle, so that it be above and parallel to 
it. 'If the connecting wire be sunk on either side of the needle, so as to come into the horizontal plane in which the needle is allowed to move, there is no motion of the needle in that plane, but the needle attempts to move in a vertical circle, and but for the imperfect suspension, and the earth's magnetism, would do so. When the wire is on the east of the needle, the pole next the negative end of the battery is elevated, and when on the west of the needle it is depressed. If the connecting wire be now sunk below the level of the needle, similar attractions and repulsions take place, but in opposite directions to those followed when it is above. The pole of the needle opposite the negative end of the battery now moves eastwards, whatever the position of the wire, so that it be restricted as above.

The nature of the connecting wire, provided it be metallic, does not materially interfere in these phenomena; a tube filled with mercury is effectual. It continues, also, though the conductor be interrupted by water, unless the interruption be of great extent. The magnetic influence of the wire also extends through all sorts of substances, as is the case with common magnetism. It does not act on needles of brass, glass, or gum lac.

If an unmagnetised steel needle be placed parallel to, and in contact, or nearly so, with the connecting wire, it acquires opposite magnetisms upon its two sides; but if it be placed at right angles to the connecting wire, it becomes polar, and is a permanent magnet. All these facts may be observed by the electricity of the common machine, but it is inconveniently em- 
ployed, in consequence of the deficiency in quantity of electricity. Those forms of the voltaic apparatus which generate quantity, are such as produce the most decided electromagnetic effects;they appear to be independent of tension, and, accordingly, a single pair of very large plates is more effectual than a numerous succession of small ones. Batteries constructed for the purpose, of one or more pairs of large plates, have sometimes been termed magneto-motors.

That attraction and repulsion may be communicated to metallic wires by the electric current alone, independent of a magnet, is shewn by placing two wires parallel to each other, and so suspended as to move freely when transmitting electricity of the battery. If the current pass along both wires in the same direction they attract, if in opposite directions they repel each other. If, also, the one wire be fixed and the other moveable, the currents being sent in opposite directions, then the moveable wire will turn round until they are in the same direction. Mr. Faraday observes, ' that the contrast between these attractions and repulsions, and those usually called electrical, are very striking.' These take place only when the circuit is completed; those, only when it is incomplete. The magnetic attractions take place between the similar ends of the wires, and the repulsions between the dissimilar ends, but the electrical attractions take place between dissimilar ends, and the repulsions between similar ends. These take place in vacuo, but those do not. When the magnetic attraction brings the two wires together, they remain in contact; but when electrical attraction 
brings two bodies together, they separate after the contact.

The voltaic pile itself acts upon a magnetic needle in the same manner as the connecting wire. When placed near a pile or trough in action, having its poles connected by a wire, it immediately moves, becoming obedient to the battery in the same manner as to the connecting wire, so that the needle becomes an instrument competent to indicate that state of an active voltaic pile, and of the wire connecting it, which is supposed to be occasioned by currents of electricity. The needle thus applied is sometimes called a galvanometer.

It has already been stated, that the connecting wire confers magnetism upon steel; that it is itself everywhere a magnet, is shewn by presenting iron-filings to any part of it, which are instantly attracted by and cluster round it, but fall off almost the instant that the connexion with the battery is broken.

Having ascertained that the action of the connecting wire on the direction of the magnet is owing to a tendency which they have to revolve round each other, Mr. Faraday contrived an apparatus by means of which either pole of a magnet was made to revolve round a wire as a fixed point. The description of this instrument, which has been the parent of a great variety of analogous revolving apparatus, is given at length in the 12th volume of the Quarterly Journal.

The phenomena which have been adverted to, may be explained by the hypothesis of Dr. Wollaston; upon the supposition of the existence of 
an electro-magnetic current or vortex passing round the axis of the wire, and apparently at right angles to the electric current. The direction of the magnetic current, as dependent upon that of electricity, is demonstrated by the affections of the approximated pole of the magnet, and by its rotatation, where it has freedom of motion; and the following contrivance of Dr. Roget is useful in assisting the memory respecting the details of these affections.

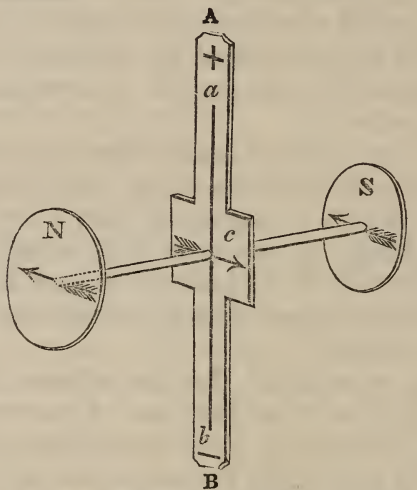

AB (see the wood-cut) is a slip of card, on each side of which a line $a b$ is drawn along the middle of its length, the end $a$ being marked + , the end $b-$, and the centre $c$, being crossed by an arrow at right angles to it directed as in the figure. Through the centre and at right angles to the plane of the slip of card, there is made to pass a slender stem of wood, at the two ends of which are fixed, in planes parallel to the slip of card $A B$, 
the circular discs of card, marked respectively with the letters $\mathrm{N}$ and $\mathrm{s}$, and with arrows parallel to, but pointing in a contrary direction to the one at c. The same marks must be put on the reverse of each of the three pieces of card, so that when held in different situations they may be seen without turning the instrument.

If the line $a b$ be supposed to represent the galvanic wire (the direction of the current of electricity being denoted by the signs + and - , at the ends of the line), the arrow at the centre will point out the direction in which it tends to move, when under the influence of the north pole of a magnet situated at $\mathrm{N}$; or of a south pole situated on the other side at $\mathrm{s}$ : and, vice versâ, the arrows at $\mathrm{N}$ and $\mathrm{s}$ will indicate the directions in which the north and south pole, respectively, tends to revolve round the galvanised wire in its vicinity, with relation to the direction of the current of electricity that is passing through it.

It must be observed that the poles $\mathrm{N}$ and $\mathrm{s}$ are here not considered as in connexion with each other, or as forming parts of one magnet: their operations are exhibited singly, and quite independently of each other. The advantage of this little instrument consists in its being capable of being held in any situation, and thus easily adapted to the circumstances of any fact or experiment of which we may wish to examine the theory.

If the conducting wire be twisted into a helix or spiral form, by rolling it round a solid rod or a glass tube, it will, in some respects, represent a polar magnet, and a piece of steel introduced into the central cavity of such a spiral, speedily 
becomes powerfully magnetic. For these purposes, wire, covered with silk, is conveniently used, as it may be closely coiled without allowing the passage of electricity from surface to surface, and it will be found that if such a coil have freedom of motion, if, for instance, it be floating about in a basin of water, it will be obedient to the poles of the magnet, mutually attracted and repelled by them, and forms what may be called a voltaic magnet. Ampère, whose theory of electro-magnetism deserves to be attentively studied*, terms such an arrangement an electrodynamic cylinder.

If a magnetic needle be surrounded by several convolutions of wire, covered with silk to prevent electric communication between its different parts, a deflection from its meridian will be occasioned by the transmission of the minutest quantity of electricity through the coil. In this way the electricity produced by the contact of metals, by their immersion in acid and other solutions, and by mere changes of their temperature, may be rendered evident. M. Schweigger, who thus first applied the magnetic needle to such detection of quantitative electricity, has termed the above arrangement an Electro-Magnetic Multiplier.-Ann. of Phil., N. S., vol. v., p. 436.

* See an able article upon this subject in the Quarterly Review, xxxv. 237. 


\section{N D E X.}

Accumulation of galvanic electricity . . . . . . . 213 Affinity Aggregation, attraction of

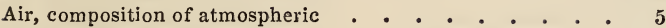
- infiuences crystallisation . . . . . . . . . 16 thermometer . . . . . . . . . . 60

- conducting power for heat . . . . . . . . 72 136 Alcalies, decomposed by electricity . . . . . . . 216 Amalgam

Amber, electricity of Analysis Andes, temperature of Anhydrous salts Animal electricity Anomalous expansion of water . Apparatus for decomposing water 60 72 
Balls and points for electrical conductors

Baryta, sulphate composition of

Battery, electrical

Beccaria on magnetism

Bismuth, crystallisation of

Bittern

Black, Dr., on the latent heat of water

Boiling in vacuo

Bolognian phosphorus

Brain, supposed electricity of

Bricks, radiate and absorb heat

Camphor, crystallisation of . . . . . . . . 18 curious combustion of

Candle, blown out by electric aura . . . . . . . 190

Canton's phosphorus . . . . . . . . . . 140

Cascariolo discovers a phosphorus . . . . . . . 139

Capacity for heat . . . . . . . . . . . 62

Carbonate of lime, its phosphorescence . . . . . . 143

Cat's back, electricity of . . . . . . . . . 164

Cavallo on the aurora borealis . . . . . . . . 291

Centigrade thermometer . . . . . . . . . . 57

Change of form, a consequence of chemical attraction.$\quad 25$

Charcoal conducts electricity

Charge of the Leyden jar . . . . . . . . . . 197

Chemical attraction . . . . . . . . . . 22

opposed to mechanical . . . . . 27

properties of metals influenced by electricity . . 212

Chemistry defined . . . . . . . . . . . 1 
Children, Mr.

Churches protected from lightning

Circle, galvanic .

Cinders

Clothes, warmth of

Clouds

prevent radiation

Coals, combustion of

Coal-mines, fire damp of

Coated pane of glass

Cold, effects of

- produced by liquefaction

231

276

213

148

60

88

137

24

157

193

76

83

123

Colour of phosphorescent light . . . . . . . . 142

- influences temperature . . . . . . . . 136

Colours, theory of . . . . . . . . . . . . 117 prismatic

Column, electric

Combustion

24,145

$$
\text { cause of }
$$

without flame

peculiar products of .

Conductors of electricity

168

66

Confervæ, vegetation of

Cryophorus

Crystals, large

obtained by solution structure of

Crystallisation, how effected theory of . uses of

Dalibard and Delor, draw electricity from the clouds

Dalton on the aurora borealis

Danger in thunder-storms

273

287

Daniell, Professor

279

Davy on radiating vessels

- the safety-lamp electro-chemical action 
Defiection of the magnetic needle by electricity

\section{Deliquescence}

Deluc's electric column

Description of the aurora borealis by Dalton . . . . 287

Dew by Parry

Discharger, electrical .

Distillation . . . .

Earth, radiation of heat by

Eel, electric .

Efflorescence

Electrical attraction and repulsion

- aura.

balls

bells

excitation

figures

fily-wheel

190

189

187

162

188

191

185

186

179

184

187

161

293

192

208

208

162

165

165

165

165

304

303

304

212

237

298

173

141

Electro.magnetism

decomposition

Electrometer

Bennet's 
Flectrophorus

Electro-polarity

Electro positive bodies

Elements, chemical

Epsom salt .

Equivalents

Ether, converted into vapour

Expansion

Expansion of air

anomalous, of water of liquids of metals

176

173 196 196 174 217 203 17 247 of solids

Falling stars 35

Faraday on electro-magnetism

Feather, electrified

Fire-damp of coal-mines

Fluor spar, phosphorescence of . 
Franklin, Dr., on conductors

Captain, on the aurora borealis

Freezing by ether . . . . . . . . . . 102

Friction, a source of electricity

Galls, a test for iron . . . . . . . . . 252

Galvani

Galvanic batteries. theory

Galvanism

Galvanometer

Glass, electricity of

214

207

301

162

- impermeable to electricity . . . . . . . 200

Glass, expansion of by heat . . . . . . . . . . 38

Glauber's salt, crystallisation of . . . . . . . . 12

Glow-worm . . . . . . . . . . . . . 144

Good radiators of heat produce cold . . . . . . . 136

Grass a good radiator of heat . . . . . . . 136

Gravel-walks, radiation of heat from . . . . . . 136

Grey, Dr., on thunder and lightning . . . . . . . 269

Gunpowder inflamed by electricity . . . . . . 261

Gymnotus . . . . . . . . . . . . 294

Halley, on the aurora borealis . . . . . . . 287

Harris, Mr, on ships' conductors . . . . . . . . 277

Heart, pulsation of . . . . . . . . . . . 297

Heat . . . . . . . . . . . . . . 33

absorption of . . . . . . . . . . 65

capacities for .. . . . . . . . . 62

changes form and state $\quad . \quad . \quad . \quad . \quad . \quad . \quad . \quad . \quad . \quad .77$

distribution through liquids . . . . . . . . 69

effect of on liquids . . . . . . . . . 86

on solids . . . . . . . . 34, 77

evolution of . . . . . . . . . . 64 of fluidity . . . . . . . . . . 86

latent . . . . . . . . . . . . 78 radiant . . . . . . . . . . . . 112 radiation of . . . . . . . . . 120 reflection of . . . . . . . . . 121 
Heat and light, their mutual relations

Ice, how made in Bengal

- produced by evaporation

Identity of different forms of electricity

Ignition

Impurities of distilled water

Incandescence

Induced electricity

Induction in the galvanic series

of electricity through glass

Inflammation of gunpowder by electricity . . . . . . 262

Insulated Leyden jar

Insulators, electrical

Intensity of electricity

Iodine, evolved at the electro-positive pole 202

Iron, protected by electro-chemical action

Latent heat

Lamp, safety

Lanthorn fly

Leaves, vary in radiating powers

- functions of

Leyden jar 
Light and heat evolved by chemical action electric . • • . . . . . . . . 192

cause of

in different media

183

185

brilliant display of . . . . . . 227

of flames

- magnetism produced by .

Lime, nitrate of phosphorescent

116

- sulphate of, decomposed by electricity

Lightning

Liquefaction, theory of

243

Liquids, conducting powers of for heat

Luminous bodies

Magnesia, sulphate crystals of

Magnet, deflected by electricity

304

- voltaic

Magnetic attraction and repulsion

- needle how affected by electricity

Beccaria on

Magnetomotor

Matter, radiant . . . . . . . . . . . 128

Metals, electric, conducting power of . . . . . . . 236

Moirée metallique

Nairne on aurora borealis

's electrical machine 
Natural electricity :

Nature of electricity . . . . . . . . . 161

Negative electricity

145

Neutral salts. . . . . . . . . . . . 26

Noise of aurora borealis . . . . . . . . . 291

Nollet on thunder . . . . . . . . . . . . 269

Nonconductors of electricity . . . . . . . . 167

enumerated . . . . 168

Ocean, luminosity of . . . . . . . . . . . . . 144

Octoedron . . . . . . . . . . . . . . . 8

Oersted on electro-magnetism . . . . . . . 298

Oil, specific heat of . . . . . . . . . . 62

Organic substances, electricity of . . . . . . . . 296

Oxygen . . . . . . . . . . . . . 5

148

supports combustion . . . . . . . . 5

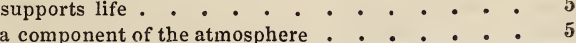

its electrical relations . . . . . . . . . 247

Oyster-shells, phosphorescence of . . . . . . . . 140

Paper becomes electrical by friction . . . . . . 164

Papin's digester. . . . . . . . . . . . . . . 39

Paradoxical experiment . . . . . . . . . . . . 92

Parry, Captain, on the aurora borealis . . . . . . 287

Pendulum compensation . . . . . . . . . . . 40

$3 y$

Perpetual motion electrical . . . . . . . . 218

Phosphate of lime phosphorescent . . . . . . . . 143

Phosphorescence . . . . . . . . . . . . . 138

—— of oyster-shells . . . . . . . . . 140

143

143

139

of sulphuret of lime . . . . . . . 140

of animals. . . . . . . . . . . 144

146

144

145

Phosphori, solar of sea-water 
Phosphori, solar, affected by friction Phosphorus, Baldwin's

\section{Photometer}

Plants, suffer by radiation

Plate of glass charged .

Plates, size of for Voltaic pile

Points, electrified

for lightning conductors

Polarity, magnetic, conferred by electricity . . . • . 299 - electrical, by induction

171

Positive and negative electricity in Leyden jar . . . . 199

Pressure, boiling under . . . . . . . . . 94

Products of combustion . . . . . . . . . . 148

Protection of copper sheathing . . . . . . . . 255

Prussiate of potassa a test for iron . . . . . . . 252

254

Pulsation of the heart . . . . . . . . . . . 297

Pyrometer . . . . . . . . . . . . . 59

Radiant matter . . . . . . . . . . . 112

heat nature of $\cdot \cdot \cdot \cdot \cdot \cdot \cdot \cdot \cdot \cdot \cdot \cdot 12$

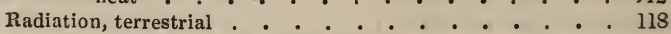

—_ economically applied . . . . . . . . 131

Rain
Ray, electric

Ray, chemical . . . . . . . . . . . .

— solar ............... . . 118 
Rays, heating power of

- relative, refrangibility of $\cdot$.

Reaumur's scale . . . . . . . . . . . 57

Receptive powers of surfaces for heat . . . . . . . 129

Red heat . . . . . . . . . . . . . 146

Red lead and sulphur separated by electricity . . . . 263

Reflection of cold . . . . . . . . . . 120

121

Repulsion, electrical . . . . . . . . . 162

Repulsive power of heat . . . . . . . . . . 33

Results of affinity . . . . . . . . . . 23

Rhomboid . . . . . . . . . . . 21

Richer on the gymnotus . . . . . . . . . 294

Richman, Professor, death of . . . . . . . 275

Rods for conducting lightning . . . . . . . . 276

Roget on electro-magnetic currents . . . . . 302

Romas on the electric kite . . . . . . . . 274

Rooms, how heated . . . . . . . . . . 133

Safety lamp . . . . . . . . . . . 158

- theory of . . . . . . . . . . 159

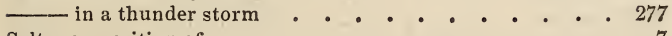

Salt, composition of . . . . . . . . . . 7

— crystallization of . . . . . . . . . 12

— anhydrous . . . . . . . . . . . 12

— deliquescent . . . . . . . . . . . 12

— effervescent . . . . . . . . . . . . . 12

- Epsom . . . . . . . . . . . . . . 12

- Glauber's . . . . . . . . . . . . . 12

- electro-chemical decomposition of . . . . . 239

Scheele on radiant matter . . . . . . . . . 115

Schweigger's electro-magnetic multiplier . . . . 304

Sea-water, corrodes copper . . . . . . . . 255

- luminosity of . . . . . . . . . . . . 144

Sealing.wax, electricity of . . . . . . . 163

Ships' conductors . . . . . . . • . • • 276

Silk, electricity of . . . . . . . . . . 164

Silurus, electrical . . . . . . . . . . . 294

Silver, action of on water . . . . . . . . . 251

- on muriatic acid . . . . . . . 253

salt-water . . . . . . . . 251

Simple and compound substances . . . . . . . . 3

— decomposition . . . . . . . . . 28 
Simple galvanic circle .

- substances.

Singer on aurora borealis

Slow combustion of alcohol of ether.

Smoky chimnies

Solution explained.

Sound, progress of .

Spectrum, prismatic

composition of

Specific heat

Spherical particles .

Spiral luminous tube

Spirit lamp, without flame

of wine, inflamed by electricity

Spontaneous phosphorescence

Star, of positive electric light

Stars, falling

192

292

292

Steam, bulk of

sections of

theory of .

Watt's

latent heat of

nature of

\section{temperature of}

Stoves, improved

Sublimation

Submarine volcano

Sulphate of baryta, composition of .

Sulphuret of copper surface influences radiation 
Table of expansion of metals

Temperatures at which bodies burn . . . . . . . 147

Test for copper

Tests.

Texture of crystals .

252

Theory of the Voltaic pile Thermometer

Tillard, Captain, on a submarine volcano

Vapour, constitution of . . . . . . . . . . . 9 91

Venezuela, gymnoti of . . . . . . . . . . . 295

Vitreous electricity . . . . . . . . . . . . . 165

Volcano, electricity of . . . . . . . . . . . . 284 submarine . . . . . . . . . . . . 285

Volta, electric pile of . . . . . . . . . . . . . 219

— theory of . . . . . . . . . . 263

Voltaic, apparatus . . . . . . . . . . . . . . . 223

— battery . . . . . . . . . . . . . . 224

magnet . . . . . . . . . . . . . 304 pile . . . . . . . . . . . . . . . 219 electricity . . . . . . . . . . . . . 207

action of on magnets . . . . . . 301 chemical effects of . . . . . . . . 235 Davy's discoveries in . . . . . . 245 
Wall, Dr. . . . . . . . . . . . . . . . . . . 269

Walls, absorb heat . . . . . . . . . . . . . 132

why wet in winter . . . . . . . . . . . 135

Warming houses . . . . . . . . . . . . 133

Water, composition of . . . . . . . . . 6, 238

decomposition of . . . . . . . . . . . . 236

apparatus for decomposing . . . . . . . , 236

boiling point of . . . . . . . . . . . 90

- latent heat of . . . . . . . . . . . . . 79

elements of, separated by electricity . . . . . . . 237

- impurities in . . . . . . . . . . . . . . 244

- anomalous expansion of . . . . . . . . 43

— greatest density of . . . . . . . . . . . . . 44

- of crystallization . . . . . . . . . . . . . 13

Water-spouts . . . . . . . . . . . . . . . . 284

Wells, Dr., on dew . . . . . . . . . . . . . . . 137

White heat . . . . . . . . . . . . . . . 147

Wilson's phosphorus . . . . . . . . . . . . . . . 140

Wine-coolers . . . . . . . . . . . . 106

— directions for cooling . . . . . . . . . . . . . 85

Wire gauze, experiments with on flame . . . . . . . 157

lamp. . . . . . . . . . . . . . . . 158

Wires, effect of electricity on different . . . . . . . . 232

Wollaston, Dr., on electro-chemical theory . . . . . . . 264

on electro-magnetism . . . . . . . . . 301

on electro-chemical decomposition . . . . . 259

on the cryophorus . . . . . . . . . . . 104

on the Voltaic apparatus . . . . . . . 225

Wood, phosphorescence of . 


\section{LONDON :}

Printed by William Clowes, Stamford Street.

$\cdot$ 




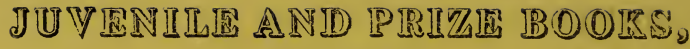
PRINTED FOR

\section{A. K. NEW M A A AD CO. IONDON. \\ MRS. HOFLAND'S WORKS.}

\section{Price Five Shillings each,}

IN ELEGANT EMBOSSED BINDINGS, WITH GILT FDGES, AND LETTERHD, ILLUSTRATED WITH PLATES.

1 AFRICA DESCRIBED, including the recent Discoveries

2 DECISION, a Tale

3 ENERGY, a Tale

4 FARE WELL TALES

5 FORTITUDE, a Tale

6 HUMILITY, a Tale

7 INTEGRITY, a Tale

8 MODERATION, a Tale

9 PATIENCE, a Tale

10 REFLECTION, a Tale

11 SELF-DENIAI, a Tale

12 YOUNG CADET, or Henry Delamere's 'Travels in Hindostan

\section{Price Two Shillings and Sixpence each,}

NEATLY HALF BOUND IN ROAN, AND ILLUSTRATED WITH PLATES.

13 AFFECTIONATE BROTHERS

14 ALICIA AND HER AUNT, or, Think before you Speak

15 BARBADOES GIRL, a Tale for Young People

16 BLIND FARMER and his Children

17 CLERGYMAN'S WIDOW and her Young Family

18 DAUGHTER-IN-LAW, her Father and Family

19 ELIZABETH and her Three Beggar Boys

20 GOOD GRANDMOTHER and her Offspring

21 MERCHANT'S WIDOW and her Young Family

22 PANORAMA OF EUROPE, continued to the present period

23 RICH BOYS AND POOR BOYS, and other Tales

24 SISTERS, a Domestic Tale

25 STOLFN BOY, an Indian Tale

26 WILLIAM AND HIS UNCLE BEN

27 YOUNG NORTHERN TRAVELLER

28 YOUNG CRUSOE, or Shipwrecked Boy

29 HOFLAND's (Mrs.) ILLUSTRATED ALPHABET, with Poctry, 26 beautiful wood cuts, square $12 \mathrm{mo}$.. fancy covers, $1 \mathrm{~s}$.

30) The same Work, coloured plates, 1s. 6d.

CIIRISTMAS TALES, HISTORICAL and DOMESTIC, by W. H. HARRISON, seven beautiful plates, embossed roan, gilt edges, is FLOWERS OF ANECDOTE, WIT, HUMOUR, GAIETY, AND

GENIUS, 18 plates, by Landseer and Heath, fancy binding, 5s.

POETIC WREATH; Select Passages from the English Poets from

Chaucer to Wordsworth, $12 \mathrm{mo} .33$ cuts, cloth lett. gilt edges, $6 \mathrm{~s}$.

GARLAND OF LOVE, wreathed of Pleasant Flowers, gathered in the

Field of Enlish Poesy, embossed velvet, gilt edges, 5s. 


\section{JUVENILE BOOKS, CONTINUED.}

HALF BOUND, ROAN, OR CLOTH, LETTERED.

ANGELINA, OR CONVERSATIONS OF A LITTLE GIRL WITH HER DOLL, numerous cuts, $2 \mathrm{~s} .6 \mathrm{~d}$.

Compendious HISTORY OF ENGLAN D, 18mo. cuts, cloth, lett.2s.6d.

CONSTANCY, a Moral Tale for Young Ladies, 3s.

COUSIN ELLEN, by Eliza Paget, fine plates, sq.12mo. cloth, lett. 2s. $6 \mathrm{~d}$.

DRAMATIC BEAUTIES; Companion to Beauties of Shakespeare, $12 \mathrm{mo}$. cloth, lettered, $3 \mathrm{~s}$.

DON QUIXOTE, abridged for Youth, 12 plates, bound in fancy cover, new edition, larger type, 1839, 3s. $6 \mathrm{~d}$.

EMBROIDERED FACTS, by Mrs. Alfred Barnard, six fine wood cuts, square $16 \mathrm{mo}$. cloth, lettered, $3 \mathrm{~s} .6 \mathrm{~d}$.

GYMNASTICS (Sports) for Boys, and CALISTHENICS (Sports)for Young Ladies, illustrated by 43 engravings, by G. Hamilton, new edition, 1840, green roan, lettered, 2 s. $6 \mathrm{~d}$.

MARIA WEST, OR SOLDIER's ORPHAN, 2s. 6 d.

MASON's CRUMBS FROM THE MASTER's TABLE, 32mo. cloth, lettered, gilt edges, $1 \mathrm{s.} 6 \mathrm{~d}$.

STORIES ABOUT THE HISTORY OF POLAND, by Robin Carver, fine plates, cloth, lettered, 2 s. $6 \mathrm{~d}$.

'IALES OF THE ACADEMY, 2 vols. 4 plates, 5s.

THE VACATION, or TRUTH \& FALSEHOOD, numerous cuts,2s6d VISIT T'O THE SEA SIDE, 18mo. cloth, lettered, 3s. 6 d.

WISEMAN's STORIES ABOUT BIRDS, numerous cuts, square $12 \mathrm{mo}$. cloth, lettered, 3s. $6 \mathrm{~d}$.

The YOUNG GEOGRAPHER, by Rev. W. Fletcher, 18mo. cloth, lettered, gilt edges, $2 \mathrm{~s} .6 \mathrm{~d}$.

The YOUNG SEER AND THE GIPSY, or early Searches into Futurity, by Eliz. F. Dagley, frontispiece, 12mo. cloth, lettered, 3s.

\section{Price Two Shillings each,}

HALF BOUND IN ROAN, OR CLOTH, LETTERED, WITH PLATES.

BIOGRAPHY OF A SPANIEL

CLAIRMONT CASTLE (Tales of)

EMILY AND HER COUSINS, by Mrs. Baker

FAIRY TALES, by Miss Selwyn, 40 cuts

HENRIETTA AND HER MAMMA

HORWOOD'S (MISS) ORIGINAL POETRY FOR CHILDREN

HUGH LATIMER, OR THE SCHOOLBOY'S FRIENDSHIP

NATIONAL PREJUDICE, a Tale

PERSEVERANCE, by the Author of True Charity, \&c.

PLANTER'S DAUGHTER AND HER SLAVE

ROSE SIDNEY

TALES OF TRUTH

ECONOMY OF HUMAN LIFE, 32mo. cloth, gilt edges, 1 s. 6 d.

GAY's FABLES, 32mo. cloth, gilt edges, 1s. 6d.

JULIA WENTWORTH, a Moral Tale, 18mo. half-bound roan, 1s. $6 \mathrm{~d}$.

JUVENILE STORY BOOK, or Tales of Affection, Benevolence,

Industry, \&c. by Louisa Stanley, 16 cuts, embossed cloth, lett. 1s. 6 d.

LITTLE DOWNY, or History of a Field Mouse, do. 1s. $6 \mathrm{~d}$.

LITTLE PRISONER, or Passion and Patience, do. 1s. $6 \mathrm{~d}$. 
